\title{
Stock market dynamics : an empirical investigation of the relationship between stock return volatility and trading volume
}

Citation for published version (APA):

Bauer, R. M. M. J. (1997). Stock market dynamics : an empirical investigation of the relationship between stock return volatility and trading volume. [Doctoral Thesis, Maastricht University]. Universiteit Maastricht. https://doi.org/10.26481/dis.19971002rb

Document status and date:

Published: 01/01/1997

DOI:

10.26481/dis.19971002rb

Document Version:

Publisher's PDF, also known as Version of record

Please check the document version of this publication:

- A submitted manuscript is the version of the article upon submission and before peer-review. There can be important differences between the submitted version and the official published version of record.

People interested in the research are advised to contact the author for the final version of the publication, or visit the DOI to the publisher's website.

- The final author version and the galley proof are versions of the publication after peer review.

- The final published version features the final layout of the paper including the volume, issue and page numbers.

Link to publication

\footnotetext{
General rights rights.

- You may freely distribute the URL identifying the publication in the public portal. please follow below link for the End User Agreement:

www.umlib.nl/taverne-license

Take down policy

If you believe that this document breaches copyright please contact us at:

repository@maastrichtuniversity.nl

providing details and we will investigate your claim.
}

Copyright and moral rights for the publications made accessible in the public portal are retained by the authors and/or other copyright owners and it is a condition of accessing publications that users recognise and abide by the legal requirements associated with these

- Users may download and print one copy of any publication from the public portal for the purpose of private study or research.

- You may not further distribute the material or use it for any profit-making activity or commercial gain

If the publication is distributed under the terms of Article $25 \mathrm{fa}$ of the Dutch Copyright Act, indicated by the "Taverne" license above, 
Stock Market Dynamics 
ISBN 90-9010790-8

○ R.M.M.J. Bauer, Maastricht 1997

Produktie en druk: Datawyse I Universitaire Pers Maastricht 


\section{Stock Market Dynamics}

An Empirical Investigation of the Relationship

between Stock Return Volatility and Trading Volume

\section{PROEFSCHRIFT}

ter verkrijging van de graad van doctor

aan de Universiteit Maastricht

op gezag van de Rector Magnificus, Prof. mr. M.J. Cohen, volgens het besluit van het College van Decanen

in het openbaar te verdedigen

op donderdag 2 oktober 1997 om 16.00 uur

door

Robert Maria Margaretha Jozef Bauer 


\section{Promotor:}

Prof.dr. C.G. Koedijk

\section{Beoordelingscommissie:}

Prof.dr. C.J.M. Kool (voorzitter)

Prof.dr. A.G.Z. Kemna

Prof.dr. Th.E. Nijman (Katholieke Universiteit Brabant, Tilburg) 


\section{Preface}

Shortly after completing my Master's degree in Economics, I joined the Finance Department of the University of Maastricht. Inspired by several research projects, I gradually decided to focus on empirical research instead of teaching. I'm especially grateful to Fred Nieuwland and Willem Verschoor, who encouraged me to write this dissertation while having fun in a shabby, dark bar in Maastricht. A large part of this thesis emerged during my stay as an assistant professor at the University of Aachen in Germany. This thesis would however not have been completed successfully without the on-going support of many people in Maastricht. First of all, I would like to mention my thesis supervisor Kees Koedijk. His comments, criticisms and motivational talks worked as an inspiration for the development of this thesis. Moreover, I would like to thank Ronald Mahieu, Roman Liesenfeld and Fred Nieuwland, who taught me the basics of modern estimation techniques. In addition, they were involved in various research projects and papers on which some of the chapters in this thesis are based.

During my work on this thesis and other research projects, I crossed a lot of virtual borders on my PC by studying various global stock markets. In order to get to my working address in Aachen, I daily crossed real country borders too. This reminds me: writing the largest part of a thesis in Germany, as a Dutchman, is quite an interesting task. It takes away most of the prejudices one might have against our neighbours. I discovered some unexpected virtues and learned that we, Dutchmen and Germans, in fact are much alike. On the sporting level however, our department occasionally experienced some "highly volatile" days, especially after a match of both national soccer teams. Luckily, this type of volatility, in contrast with stock market volatility, did not have a memory lasting longer than one working day. I would like to thank all my German colleagues and friends for their support and patience during the completion of this thesis. First of all, Thomas Hartmann-Wendels, who provided a perfect setting to work on this thesis and several other research projects in his department. Then of course my colleagues during my stay in Aachen: Andrea Das Gupta, Stefanie Dickhut, Martina Gumm-Heußen, Thomas Spicher, Thomas Tschugmell, Claudius Vievers, Werner Winkens and a whole bunch of assistants ("hiwis"), who all contributed to this thesis in one way or the other.

Inquire Europe is gratefully acknowledged for granting financial research support for this project. Moreover, I would like to thank Beursdata B.V., an affiliate of the Amsterdam Stock Exchanges, for providing an intra-daily data set used in Chapter 6. A special word of thanks goes to ABP Equity Investments, my current employer, for providing the opportunity and freedom to let me finish this thesis smoothly. Finally, I would like to thank Suzanne, who supported and motivated me during the years. 


\section{Contents}

CHAPTER 1

Aims and Scope

1.1 Introduction

1.2 Outline of the Thesis

CHAPTER 2

Volatility and Volume: a Literature Overview

2.1 Introduction 19

2.2 AutoRegressive Conditional Heteroskedasticity Models 22

2.3 Stochastic Volatility Models 25

2.4 The Mixture of Distributions Hypothesis 27

2.5 Empirical Tests of the MDH 32

2.6 The Market Microstructure Literature 36

2.7 Concluding Comments 38

CHAPTER 3

German Stock Market Dynamics

3.1. Introduction 41

3.2 Data and Summary Statistics $\quad 42$

3.3 Modelling German Stock Market Dynamics: Methodology 47

3.4 Estimation Procedures and Empirical Results 50

3.5 Concluding Comments 55

Appendix A: Markov Chains 56

CHAPTER 4

A Multiplicative GARCH Model for Volatility and Volume

4.1 Introduction 59

4.2 The Mixture of Distributions Hypothesis and GARCH 61

4.3 Limitations and Alternatives of the MDH 65

4.4 Data and Summary Statistics $\quad 70$

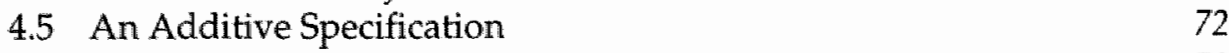

4.6 A Multiplicative Specification $\quad 79$ 
4.7 Lower Frequency (Weekly) Results

4.8 Interpretation of Results and Concluding Comments

Appendix A: Derivation of the Multiplicative GARCH Model " : 83

Appendix B: Integrating out the Mixing Variable 85

Appendix C: List of Company Abbreviations 86

\section{CHAPTER 5}

Embedding Volume as a Scale Factor in a SV Model

5.1 Introduction

5.2 The Concept of Stochastic Volatility 89

5.3 Stochastic Volatility and Volume 91

5.4 Data Description and Summary Statistics 96

5.5 Sign Bias Tests 99

5.6 VAR Models and Granger Causality Tests 104

$\begin{array}{lll}5.7 & \text { Estimation Results } & 110\end{array}$

5.8 Interpretation of Results and Concluding Comments 114

Appendix A: Impulse Response Functions

\section{CHAPTER 6}

Volatility and Volume: an Intra-Daily Perspective

6.1 Introduction

6.2 Data Description and Summary Statistics 121

6.3 Univariate Stochastic Volatility Models 128

6.4 Univariate and Bivariate Mixture Models 132

6.5 Estimation Results for the Mixture Models 134

6.6 Interpretation of Results and Concluding Comments 137

Appendix A: Construction of the Data Set 139

Appendix B: Simulated Maximum Likelihood Estimation (SML) 139

Appendix C: The Accelerated Gaussian Importance Sampler (AGIS) 143

\section{ChAPTER 7}

A Bayesian Analysis of Stock Return Volatility and Trading Volume

7.1 Introduction

7.2 The Theoretical Model 149

7.3 The Empirical Model 151

7.4 The Estimation Procedure 154

7.5 Data Description and Summary Statistics 157

7.6 Univariate Simulation Results 
7.7 Bivariate Simulation Results

7.8 Interpretation of Results and Concluding Comments

Appendix A: Markov Chain Monte Carlo Methods

CHAPTER 8

Concluding Comments and Suggestions for Future Research

REFERENCES

NEDERLANDSE SAMENVATtING 


\section{List of Tables}

3.1 Summary Statistics of Weekly Returns $(12 / 1 / 73-25 / 3 / 92)$

3.2 Summary Statistics of Monthly Returns $(12 / 1 / 73-25 / 3 / 92)$

3.3 ARCH Test Results for Weekly Returns 47

3.4 ARCH Test Results for Monthly Returns 47

3.5 GARCH(1,1)-Normal Estimates of Weekly Returns 52

3.6 GARCH(1,1) $t$-distribution Estimates of Weekly Returns 52

3.7 GARCH(1,1)-Normal Estimates of Monthly Returns 52

3.8 GARCH(1,1) $t$-distribution Estimates of Monthly Returns 53

3.9 Generalized Likelihood Ratio Tests, Weekly Returns 54

3.10 Generalized Likelihood Ratio Tests, Monthly Returns 54

3.11 Diagnostics for the GARCH $t$-distribution Models, Weekly Returns 54

3.12 Diagnostics for the GARCH $t$-distribution Models, Monthly Returns 54

4.1 Cross-Security Medians of Summary Statistics of Daily Data 70

4.2 GARCH Normal Estimates $\quad 71$

4.3 GARCH $t$-distribution Estimates 72

4.4 GARCH Normal + Contemporaneous Volume Estimates 73

4.5 GARCH $t$-distribution + Contemporaneous Volume Estimates 74

4.6 GARCH Normal + Number of Transactions Estimates 75

4.7 GARCH $t$-distribution + Number of Transactions Estimates 76

4.8 GARCH $t$-distribution + Lagged Volume Estimates (Multiplicative) 77

4.9 GARCH $t$-distribution + Contemp. Volume Estimates

(Multiplicative)

4.10 EGARCH Normal + Contemporaneous Volume Estimates (Multiplicative)

4.11 GARCH Restricted Models $t$-distribution Estimates 80

4.12 Diagnostics GARCH Multiplicative Model

Contemporaneous Volume

4.13 Weekly Aggregated Volume Median Estimates 81

5.1 Summary Statistics Adjusted Stock Returns 98

5.2 Summary Statistics Adjusted Log Trading Volumes 98

5.3 Summary Statistics Adjusted Log Squared Adjusted Returns 100

$\begin{array}{lll}5.4 & \text { Sign Bias Tests } & 102\end{array}$

5.5 Leverage Regressions 104

$\begin{array}{ll}5.6 & \text { Optimal VAR Orders } \\ 506\end{array}$

5.7 Granger Causality Tests: Volume and Returns 109

5.8 Granger Causality Tests: Volume and Volatility 109

5.9 A Basic Stochastic Volatility Model 111

5.10 Stochastic Volatility with Leverage 111

5.11 Stochastic Volatility and Volume 112

5.12 Stochastic Volatility and Volume with Leverage 112

5.13 Multiplicative GARCH and Volume Results 114

5.14 Multiplicative EGARCH Results 114 
6.1 Hourly Turnover, Trading Volume and the Number of Official Price Changes

6.2 Mean Returns, Mean Volatility and Mean Volumes per Trading Period

6.3 Surnmary Statistics Adjusted Hourly Returns $\quad 126$

6.4 Summary Statistics Adjusted Hourly Trading Volumes : : $\quad 126$

6.5 Granger Causality Tests 127

6.6 Summary Statistics Adjusted Transformed Returns 128

6.7 Stochastic Volatility Model Estimates 131

6.8 Stochastic Volatility Model Estimates with Leverage 131

6.9 Stochastic Volatility and Volume Model Estimates 131

6:10 Stochastic Volatility and Volume Model Estimates with Leverage 131

6.11 The Static Univariate Mixture Model (Clark) 136

6.12 The Dynamic Univariate Mixture Model (Clark) 136

6.13 The Static Bivariate Mixture Model (Tauchen and Pitts) 137

6.14 The Dynamic Bivariate Mixture Model (Tauchen and Pitts) 137

7.1 Summary Statistics of the IBM Stock Return and Detrended Trading Volume Series

7.2 Summary Statistics Univariate Simulation Results 161

7.3 Summary Statistics Bivariate Simulation Results 163

7.4 Summary Statistics Univariate and Bivariate Volatility Series 166

\section{List of Figures}

3.1 The FAZ Index: Weekly Prices, Returns, Absolute Returns 46 and Squared Returns

5.1 Unadjusted and Adjusted Returns (\%) and Volumes for Hewlett Packard (1967-1987)

5.2 IRF Functions: Volatility and Volume 116

5.3 IRF Functions: Returns and Volume 117

6.1 Hourly Returns, Squared Returns, Trading Volume (in Thousands) and the Number of Transactions for Philips 122

7.1 Time Series of IBM Stock Returns and Trading Volume (1973-1991) 158

7.2 Histograms of IBM Stock Returns and Trading Volume Series (1973-1991)

7.3 Histograms of Simulated Parameters and Corresponding Sequence of Draws, Univariate SV Model

7.4 Histograms of Simulated Parameters and Corresponding Sequence of Draws, Modified Mixture Model

7.5 Scatter Diagrams Bivariate Model

7.6 Estimated Volatility Series Univariate Model, Bivariate Model and Difference 


\section{CHAPTER 1}

\section{Aims and Scope}

\subsection{Introduction}

Since the start of the extensive research in the behaviour of stock markets, for decades most academic attention was given to the perhaps most important variable in this speculative market: the stock price. Inspired by the pioneering work of Bachelier, who introduced the Random Walk hypothesis for security price changes at the start of this century, and the Modern Portfolio Theory developed by Markowitz in the 1950s, Mandelbrot (1963) and Fama (1965) published path-breaking studies on the behaviour of stock prices. Subsequently, using all the theoretical and empirical insights at that time, Fama (1970) introduced the concept of market efficiency, which is still one of the cornerstones of modern finance theory. At the same time another important and easily observable variable in the stock market, the number of shares traded or trading volume, did only play a minor role in finance. We believe that we can only really understand the behaviour of stock prices and stock markets in general, if we combine the insights of price and volume studies. In particular, we will investigate the relationship between the variability of the price process and the corresponding trading activity in international stock markets. In this thesis we conduct empirical research in various well-known global stock markets: the New York Stock Exchange, the Frankfurt Stock Exchange and the Amsterdam Stock Exchange.

Most trading volume studies in this era empirically investigated the relationship between stock prices and trading volume. Early examples are papers by Osborne (1959), Ying (1966) and Crouch (1970). In the following decade, motivated by the old Wall Street adage: "it takes volume to make prices move", research was shifted to a mainly empirical investigation of the correlation of (absolute) price differences and trading volume. Karpoff (1987) presents an excellent and complete survey of this literature. The theoretical explanation for the observed price-volume relationship was studied by Copeland (1976) in a model with sequential arrival of information. In this model information for a given stock is disseminated to only one trader at a time, which implies a positive correlation between the absolute price change of the stock and the corresponding trading volume. This model has been extended in a later study by Jennings, Starks and 
Fellingham (1981) and Morse (1980). A competing theory to explain the positive correlation between trading volume and absolute price changes is originated from the research into the distribution of speculative prices, presented by Clark (1973) and further developed by Tauchen and Pitts (1983) and Harris (1987). In the eighties the market microstructure literature was introduced. The central issue in the bulk of these studies was the interaction of several variables in the stock market as prices, bid-ask spreads, trading volume and the number of transactions, see for instance Kyle (1985) and Admati and Pfleiderer (1988, 1989). The main result of these theoretical approaches is the explanation of several empirical facts in intra-daily data, for instance patterns in volatility and trading volume during the day. Trading volume plays a very important role in the majority of these models.

In the last decade finance theory was heavily influenced by the modelling of return volatility. A variety of specifications for the volatility process and accompanying estimation procedures were presented, taking into account the wellknown clustering phenomenon already observed by Mandelbrot (1963): periods of high (low) return volatility tended to be followed by periods with high (low) return volatility. This literature however lacked a profound economic explanation of the clusters in volatility. This led to several papers in the empirical literature explicitly looking for the source of this phenomenon, see for instance Lamoureux and Lastrapes (1990a), Laux and Ng (1993) and Locke and Sayers (1993). In this part of the empirical literature the role of trading volume - next to other measures of the trading activity in the stock market - became a prominent one. In most cases it was used as a proxy variable for the varying rate of information arrival in the stock market. In particular, these studies provide evidence that serial dependence in the unobservable information process governing stock markets possibly leads to the observed clusters in the volatility process. Recently, a study by Andersen (1996) has provided a promising theoretical framework in which the insights of the volatility models above are combined with those of the previously mentioned market microstructure models. The strength of Andersen's study is that it fills up the gap between theoretically oriented market microstructure papers and empirically oriented price-volume studies.

The main objective of this thesis is to provide an empirical analysis of the relationship between stock return volatility and trading volume. In Chapter 2 we present the volatility models and introduce the discussion on the link between volatility and trading volume in greater detail. In Chapters 3 through 5 we focus on an univariate modelling framework, where trading volume is interpreted as a time-varying scale factor for volatility. We include trading volume in this type of volatility models in several ways. In the last part of this thesis, Chapters 6 and 7 respectively, we focus on an alternative but intuitively more appealing bivariate framework. Again, trading volume is incorporated explicitly in these 
models, but instead of a univariate setting we use a bivariate model for volatility and volume, where both variables are influenced simultaneously by the unobservable rate of information arrival. Throughout this thesis trading volume will be considered a useful variable for the understanding of stock market dynamics and in particular the return volatility process. In general we contribute to the existing literature by using different model specifications, estimation procedures and data sets respectively. In the next section we will present the main research objectives of each chapter of this thesis in further detail.

\subsection{Outline of the Thesis}

As documented first by Clark (1973) and further developed by Tauchen and Pitts (1983), Harris (1987) and Karpoff (1987), the link between stock returns, the volatility of stock returns and trading volume is critical to the debate about the empirical distribution of asset returns. Empirical studies on the distribution of stock returns clearly showed that these returns appear kurtotic relative to the normal distribution. A well-known theory able to explain this phenomenon is the Mixture of Distributions Hypothesis (henceforth MDH). In the MDH the return distribution is kurtotic because the data are sampled from a mixture of distributions that have different conditional variances. Clark (1973) and Harris (1987) show that the different behaviour of price changes is due to the varying rate of information arrival. On trading days without so-called "news" events we observe moderate trading activity and therefore a slowly evolving price process. On more turbulent trading days, accompanied with unexpected news events, the price process evolves much faster.

The MDH now states that both price changes and trading volumes are influenced by the same unobservable mixing variable: the rate of information arrival. in the stock market. As a consequence, the joint distribution of price changes and volume is approximately bivariate normal conditional on the latent infor. mation process. Harris (1987) shows that the MDH implies that the distribution of daily price changes displays excess kurtosis and that the distribution of trading volume is positively skewed. Furthermore it is able to explain several stylized facts found by empirical researchers on the stock price-volume relationship. Examples are a positive relation between the daily amount of shares traded and the daily absolute price change and daily price changes per se, respectively. The $\mathrm{MDH}$ was tested in several studies and in most cases its predictions are confirmed. It plays a crucial role throughout this thesis: it is used as an anchor for the discussion on the link between the variability of stock returns and the corresponding trading activity. 
The outline of this study is therefore as follows: in Chapter 2 we introduce the discussion on the relationship between volatility and volume. In order to understand the volatility process of stock markets we first introduce two classes of volatility models, conditional heteroskedastic models and stochastic volatility models. Conditional heteroskedastic models are best represented by the AutoRegressive Conditional Heteroskedastic (ARCH) models, initially developed by Engle (1982) and generalized (GARCH) by Bollerslev (1986). The empirical literature on stock markets has rendered a lot of evidence, see for instance Fama (1965) and Mandelbrot (1963), that the empirical distribution of financial asset returns exhibits leptokurtotic behaviour. In addition, we observe the before mentioned clustering phenomenon on stock markets: volatility tends to be clustered together. Trading days with a lot of price variability are followed by trading days with more than average volatility. This is a clear indication that the underlying risk in stock markets, measured as the standard deviation of returns, is timevarying. If the volatility of returns is proportional to the unobservable rate of information arrival, as stated by the $\mathrm{MDH}$, it is interesting to look at the trading activity on the stock market, measured by trading volume or the number of transactions. GARCH models have proven to be a very good fit for many financial return series, see Bollerslev, Chou and Kroner (1992). In a GARCH framework volatility shocks persist over time, which is consistent with the observed clustering in volatility. The typical GARCH specification and its empirical implications are discussed in Chapter 2 in greater detail.

The competing class of stochastic volatility (SV) models emerged from the continuous time finance literature, mainly on topics like dynamic portfolio diversification and option pricing. In this alternative conditional volatility model the changing variance of the price changes is treated as an unobservable variable following a stochastic process, mostly of an autoregressive nature. Taylor (1994) shows in his so-called information counting model that the latent volatility process in a SV model can be interpreted as the unobservable information flow. Here the link with the MDH becomes clear: SV models can be used to estimate the varying information process in speculative markets. Chapter 2 also provides a more detailed look on the MDH, its extensions and its empirical implications. In addition, we discuss the empirical studies that tested the predictions of the $\mathrm{MDH}$ and we introduce the relationship between volatility and trading volume in a MDH context. A last but important issue in Chapter 2 is the introduction of the market microstructure literature and in particular the modified mixture model by Andersen (1996). This modified version of the $\mathrm{MDH}$ is built on a theoretical framework originated from the market microstructure literature. The most important modification relative to the standard $\mathrm{MDH}$ Andersen (1996) makes, is the decomposition of daily trading volume into two separate parts. One is trading volume based on information and the other is trading volume not related to the information process. In the market 
microstructure this last part is commonly referred to as noise or liquidity trading; see for instance Kyle (1985) and Admati and Pfleiderer (1989). Examples of liquidity traders are for instance large institutional investors who trade for liquidity reasons on a certain day, often the beginning or end of the month. This generates trading volume, but we can easily assume that this is not necessarily related to the information process driving stock prices. Therefore we expect a different relationship between trading volume of this kind and trading volume induced by information events.

Chapter 3 serves as a more detailed introduction in the GARCH literature by presenting some of the well-known model specifications used in empirical studies. In addition, we aim to provide extensive statistical evidence for the presence of clustering in volatility in German stock market indices. In particular, the observed leptokurtosis may be explained by different types of GARCH models. Therefore we estimate several univariate GARCH specifications, including the GARCH-in-mean specification, proposed by Engle, Lilien and Robbins (1987). Furthermore we address the choice of the conditional error distribution. We look at two possible candidates: the normal distribution and the student $t$-distribution. The purpose of this chapter is merely to introduce the methodology and the accompanying estimation procedure (Maximum Likelihood Estimation) of GARCH modlels, which are also used in some of the subsequent chapters. The empirical results can form a body of evidence which can serve as a frame of reference for further research in the German stock market.

In Chapter 4 we propose a new, multiplicative GARCH model for volume and volatility, for German individual stock returns. The motivation for this type of model lies in the fact that economic models, which can generate or explain the observed clustering phenomenon captured by GARCH, are difficult to find. In our opinion the source of GARCH in stock returns have to be identified if we are to make any progress in the time series modelling of financial data. We first use the standard MDH of Clark (1973) as a potential explanation for the GARCH effects in the stock market, largely inspired by the empirical work of Lamoureux and Lastrapes (1990a). In their study daily trading volume is included as a proxy for the unobservable mixing variable in the conditional variance equation of a GARCH model in an additive fashion. The empirical results clearly indicated that GARCH effects tend to fade away after the inclusion of trading volume. This study therefore was a (although indirect) test of the MDH. Their methodology however was criticized in the literature, see for instance Bollerslev, Chou and Kroner (1992). We will address these and other issues in our derivation of multiplicative GARCH model. In this model we investigate a univariate multiplicative relationship between trading volume and the conditional variance of stock returns. The specification used is similar to the specification used for interest rates by Brenner, Harjes and Kroner (1993) and Koedijk, 
Nissen, Schotman and Wolff (1994). We interpret this model as being loosely inspired by the $\mathrm{MDH}$, where we relax or replace some of the original assumptions. As a result we obtain a multiplicative GARCH specification that assumes a different role for trading volume: a scale factor for volatility. As a benchmark we also estimate the additive specification used by Lamoureux and Lastrapes (1990a) using both volume and the daily number of transactions as a proxy for the rate of information arrival. The estimation, using Maximum Likelihood procedures, is conducted on a sample of 30 German stocks which are all part of the well-known DAX-index.

In Chapter 5 we use the class of stochastic volatility (SV) models to test the relationship between volatility and trading volume. First, we introduce the concept of stochastic volatility, its derivation and the possible estimation procedures. Then we show that stochastic volatility models are in fact closely related to the $\mathrm{MDH}$. The conditional variance in a SV model can be interpreted as a transformed information arrival process. We use a univariate SV specification where, analogous to Chapter 4 , trading volume is included as a time-varying scale factor for volatility. Moreover, we look for evidence of the leverage effect: Black (1976) and Hamilton (1994) show that the variance of stock returns reacts asymmetrically to positive and negative shocks in returns. Several sign bias tests are performed in order to investigate whether there is a difference in the impact of positive and negative shocks in return to volatility, see for instance Engle and $\mathrm{Ng}$ (1993). We account for the leverage effect in our SV specification by using a framework of Harvey and Shephard (1994). Here we apply a QuasiMaximum Likelihood estimation method based on the Kalman filter here. The estimation of SV models is a point of discussion in the literature. QML is a simple and straightforward way of estimating the parameters of a SV model. Although other methods could provide more efficient parameter estimates, this is no point of interest in this chapter. In addition, we present some additional evidence on the dynamics between volume and volatility by estimating Granger Causality tests, simple Vector AutoRegressive (VAR) models and corresponding impulse response functions. The data set used in this section contains a sample of stocks listed on the New York Stock Exchange and the associated S\&P 500 index. This set has been used before in a related study by Lamoureux and Lastrapes (1994), which gives us the possibility to compare our estimation results with theirs directly.

Chapter 6 looks from a different angle at the link between volatility and volume. First, we use intra-daily price and volume data obtained from the Amsterdam Stock Exchange in this chapter. Second, we use a principally different concept for the price-volume relationship. In Chapters 4 and 5 we interpreted trading volume as a proxy for the rate of information arrival, being a timevarying scale factor for conditional volatility. All estimated models were there- 
fore specified in an univariate setting. The standard MDH, see Harris (1987), however states that both price changes and trading volume are simultaneously influenced by the same mixing variable, the rate of information arrival. This implies that trading volume has no role as a proxy for information arrival in this setting. Therefore we alternatively estimate a bivariate mixture model for price changes and trading volume, as proposed by Tauchen and Pitts (1983) and Harris (1987). In their model specification, prices and volume are directed by a serially independent mixing variable. We contribute to this literature by relaxing the assumption that the mixing variable is independent. The estimation procedure we use is the Simulated Maximum Likelihood (SML) method, see Danielsson and Richard (1993). This approach relies on Monte Carlo techniques to estimate the high-dimensional integrals in the dynamic case. Serving as a benchmark, we will estimate the univariate model for price changes, which has been proposed by Clark (1973).

Chapter 7 is fully dedicated to the modified mixture model of Andersen (1996). We start with presenting the modified mixture model and its underlying theoretical market microstructure model. In the last decade a lot of studies in the market microstructure literature, often triggered by the early work of Kyle (1985), focused on the relationship between volatility and trading volume. Important variables in most of these papers are trading volume and the number of transactions. Most work is however devoted to the intra-daily relationship between volatility and volume and often from a theoretical perspective. Andersen (1996) combines the intuitively appealing features of the microstructure models - for instance the existence of liquidity traders and an asymmetric information structure - with the main characteristics of the MDH. This leads to the so-called Modified Mixture Model: a bivariate specification for prices and volumes with a fully dynamic, stochastic volatility specification for the mixing variable. In the estimation stage, Andersen (1996) uses the Generalized Method of Moments (GMM) technique to estimate the empirical specification. We will use a different technique by using Markov Chain Monte Carlo methods (MCMC). This estimation procedure has been used in a univariate SV setting by Jacquier, Polson and Rossi (1994). A big advantage of this method is that it enables us to study the latent information process, valuable in for instance dynamic portfolio allocation and option pricing. In order to be able to compare our results directly with those of Andersen (1996), we asked him to provide us with the data set (the IBM common stock price and volume series) used in his study. Therefore we finish this chapter with a direct comparison of results.

Finally, Chapter 8 will give concluding comments on the empirical results of this thesis and a list of possible research objectives for the future. 


\section{CHAPTER 2}

\section{Volatility and Volume: a Literature Overview}

\subsection{Introduction}

In this chapter we introduce the discussion on the link between stock return volatility and trading volume. Modelling and estimating the volatility process has been an important issue in many theoretical and empirical papers in the finance literature. Various models and estimation procedures have been proposed, estimated and discussed. The universe of volatility models can be divided into two related sub-groups, see Andersen (1994), Taylor (1994) and Mahieu (1995). The first one is the class of conditional heteroskedastic models, represented by the AutoRegressive Conditional Heteroskedastic (ARCH) models of Engle (1982) and the generalized specification (GARCH) of Bollerslev (1986). The second class is formed by the Stochastic Volatility (SV) models, initially developed and tested in the option pricing literature, see Taylor (1994) for a literature overview. Andersen (1994) presents a unified framework of volatility by introducing the class of Stochastic AutoRegressive Volatility (SARV) models. Both GARCH and SV models belong to this universe of models under various restrictions of parameters, see also Mahieu (1995) ${ }^{1}$. The main objective of this chapter is to start the discussion on the latent process that both drives stock return volatility and another important factor in the (dis-)equilibrium dynamics of stock markets: the number of shares traded or trading volume.

The relationship between stock returns, the volatility of stock returns and the corresponding trading volume has been on the research agenda of financial economics for almost three decades now. Karpoff (1987) presents various reasons why this relationship is important. Research concerning these dependencies is especially relevant in the debate on the empirical distribution of speculative prices, see Clark (1973). In addition, insight in the joint distribution of prices and trading volume is relevant in event studies that are based on price

In particular, Andersen (1994) presents a way to write both GARCH and SV models in the SARV-specification. Subsequently, he is able to compare these models directly. For details, see Andersen (1994). pp. 81-86. 
and volume data, see for instance Richardson, Sefcik and Thompson (1986). Moreover, Karpoff (1987) shows that the link between stock prices and volumes can have significant implications for research into options and futures markets. Finally, it is critical in the discussion in the relatively new research area called market microstructure. Kyle (1985), Karpoff (1986, 1987), Hufman (1987, 1992), Admati and Pfleiderer $(1988,1989)$ and Foster and Viswanathan $(1990,1993 a$, $1993 \mathrm{~b}, 1994,1995$ ) are prominent examples of studies in which the way new information is processed in financial markets is the main topic of research. These approaches often focus on different information structures and in some cases assume strategic behaviour of informed, e.g. Kyle (1985) or uninformed, e.g. Admati and Pfleiderer $(1988,1989)$, traders. Trading volume is a very important variable in the majority of these models.

Various theoretical and empirical studies in the seventies and the eighties across all financial markets have focused on the correlation between the absolute price change and trading volume, the price change per se and trading volume, and (conditional) volatility and trading volume. ${ }^{2}$. In the last decade econometricians discovered the fruitful area of research into the dynamics of volatility and volume on financial markets, which led to a considerable amount of published papers in this area. The main objective of the bulk of this research, where trading volume (or other measures for trading activity) is treated as a variable with information content, was to find an economic explanation for the existence of persistence in volatility in financial markets, see for instance Lamoureux and Lastrapes (1990a), Laux and Ng (1993) and Locke and Sayers (1993).

Basically, one can identify two major strands in the relevant literature. The first focuses on the co-movement of stock prices and trading volume. Clark (1973) started this theoretical analysis by introducing the so called Mixture of Distributions Hypothesis (MDH), which explores the idea of using trading volume as a proxy for some subordinated stochastic process of information arrival. This idea has been extended and refined by Epps and Epps (1976), who use another version of the mixture model, and Tauchen and Pitts (1983), who point out the possible problems in the simultaneous determination of volume and volatility. The empirical pendants of these theoretical analyses all find a strong contemporaneous correlation between stock return volatility and trading volume movements, see Tauchen and Pitts (1983), Harris (1986, 1987), Smirlock and Starks (1988) and Richardson and Smith (1994). The empirical facts in these studies clearly indicate that the return volatility process and the dynamic process that drives trading volume are heavily linked together, possibly by the same under-

2 For an excellent survey of the then existing literature on the price-volume relationship, see Karpoff (1987). In this thesis our main objective is to explain the relationship between stock return volatility and trading volume. 
lying driving force. The mixture of distributions hypothesis is able to give an elegant and intuitively appealing explanation for this phenomenon. Therefore it is used in the centre of our research throughout this thesis. The discussion on the relationship between volatility and trading volume was triggered by numerous papers on the aforementioned GARCH-model, a member of the class of conditional heteroskedastic models. Lamoureux and Lastrapes (1990a) and Laux and $\mathrm{Ng}$ (1993) are examples of empirical studies that combine the MDH with a GARCH framework. Here several proxies for the unobservable, underlying rate of information arrival are used: trading volume, the number of price changes and the number of transactions. Other papers, see Ghysels and Jasiak (1994), Lamoureux and Lastrapes (1994) and Andersen (1996) use a stochastic volatility framework to test their theory. In section 2.5 we will elaborate on this research.

Recently, the price-volume relationship has also been shown to be important to the understanding of the microstructure of financial markets. Several authors have focused on the way new information arrives at markets, which different kinds of traders are active in these markets and which information structures typically exist. Theoretical models, centred around the market microstructure model of Kyle (1985), were developed in order to understand the temporal patterns in stock return volatility and volume. Admati and Pfleiderer (1988, 1989) contribute to this discussion by providing a theory of intra- and inter-day variations in trading volume and price variability. Foster and Viswanathan $(1990,1993 a, 1993 b)$ provide a similar model and additionally study the effect of public information and competition on volatility and volume. Sometimes, in close interaction with their theoretical counterparts, empirical studies by Wood, McInish and Ord (1985), French and Roll (1986), Jain and Joh (1988) and Stoll and Whaley (1990) among numerous others, attempt to detect temporal patterns in stock return variances and volumes. Well-known results of this research are the $U$-shaped patterns in volatility and trading volume during the trading day, the inverted $U$-shape in trading volume across days and the significant differences in return volatility across trading days.

A relatively new approach is offered by Andersen (1996). In his modified mixture model, the main features of the $\mathrm{MDH}$ are combined with a market microstructure model with an asymmetric information structure, based on a theoretical framework of Glosten and Milgrom (1985). Their model is particularly suitable as it is explicitly structured to explain the process of information arrival and assimilation that occurs shortly after a piece of relevant information enters the market. In the empirical version of this model, introduced by

3 Hufman (1987), Karpoff (1986) and Wang (1994) present altemative models under different assumptions. 
Andersen (1996), it is assumed that trading volume is Poisson distributed with an informed component and a noisy component. The noisy component can be interpreted as the amount of liquidity trading in that particular stock. The existence of liquidity or uninformed traders is consistent with most market microstructure models; see for instance Kyle (1985).

In this chapter we will review every branch of the relevant existing literature in greater detail. First, we will briefly discuss the two proposed classes of volatility models in sections 2.2 and 2.3 respectively. In section 2.4 we present the pioneering work of Clark (1973) by introducing the Mixture of Distributions Hypothesis. Furthermore we summarize the subsequent studies that used his idea, among which are Tauchen and Pitts (1983) and Harris (1987). In section 2.5 we focus on empirical, econometrically oriented studies into the link between volatility and trading volume. We make a distinction between studies directly based on the MDH and studies only indirectly or even not linked to the MDH. In section 2.6 we further elaborate on the market microstructure literature and in particular on the Glosten and Milgrom (1985) model. We include this part of the finance literature as an introduction to the empirical analysis in Chapter 7, inspired by the work of Andersen (1996). Finally, section 2.7 contains concluding comments.

\subsection{AutoRegressive Conditional Heteroskedasticity Models}

In the 1960s several economists, see for instance Fama (1965) and Mandelbrot (1963), pointed out that the risk premia in stock markets may be time-varying. Variances and covariances of stock returns appeared not to be stable through time. This had a big impact on the existing asset pricing theories like for instance the Capital Asset Pricing Model (CAPM): changing risk premia lead to changing expected returns. Time-varying second moments also had a big impact on the option pricing literature: the process that describes the volatility of the underlying asset now had to be modelled stochastically. The conditional heteroskedastic class of volatility models, introduced by the path-breaking papers of Engle (1982) and Bollerslev (1986), implies that forecasts based on all available information are more efficient than forecasts not using this information. In the (G)ARCH model the conditional variance $h_{t}$ of a series of stock returns can be represented by a simple function of all information known at time $t-1$. The general structure of the conditional variance in a (G)ARCH model can then be summarized as follows: 
$h_{t}=E_{t-1}\left(\varepsilon_{t}^{2}\right)=\alpha_{0}+\sum_{j=1}^{p} \beta_{j} h_{t-j}+\sum_{i=1}^{q} \alpha_{i} \varepsilon_{t-i}^{2}, \quad \alpha_{0}>0, \quad \alpha_{1}, \beta_{1} \geq 0$ 2.2

where:

$\varepsilon_{t} \mid \Psi_{t-1} \sim D\left(0, h_{t}\right)$

Here $\Psi_{t-1}$ is the $\sigma$ field generated by $\left(\varepsilon_{t-1}, \varepsilon_{k-2}, \ldots\right\}$ containing all information available at time $t-1, y_{t}$ represents the rate of return defined as the difference in logarithmic value of two consecutive price observations, $\mu$ denotes the conditional mean, $h_{t}$ represents the conditional variance and $D$ can be any symmetric conditional distribution. Positive conditional volatility is ensured if the $\alpha_{i} \beta_{i} \geq 0$ and $\alpha_{0}>0$ and covariance stationarity is guaranteed if:

$$
\sum_{i=1}^{q} \alpha_{i}+\sum_{j=1}^{p} \beta_{i}<1
$$

Engle's ARCH model is now obtained for $p=0$. Most empirical studies however showed that a longer lag structure for the squared residuals in 2.2 was needed and therefore Bollerslev (1986) proposed the parsimonous, generalized GARCH $(p, q)$ model where $p \neq 0$. It turned out that the GARCH $(1,1)$ model captures the dependence in the conditional variance well enough. As is documented extensively in the empirical finance literature, empirical distributions exhibit leptokurtic behaviour and clusters of low and high volatility. The conditional heteroskedastic class of models has been shown to provide a very good fit for most financial return time series, see Bollerslev, Chou and Kroner (1992) for a detailed overview of the ARCH and GARCH literature. The main feature of this class of models is that an autoregressive structure on the conditional variance $h_{t}$ is imposed, parameterized as a linear function of past squared innovations and lagged conditional variances (in the generalized GARCH case), allowing volatility shocks to persist over time. This persistence captures the propensity of large absolute returns to cluster in time. Furthermore it favours the evidence often found in empirical studies that the assumption of conditional normality does not capture all of the excess kurtosis observed in high frequency stock returns. Plausible alternatives for the conditional normal distribution are

4 Note that the GARCH process is a discrete time process, but it will produce a correct estimate of the conditional variance when the underlying process is a diffusion process with stochastic volatility, provided the time interval is short enough, see Nelson (1992) for this result. 
the student $t$-distribution and the generalized error distribution (GED), see for instance Nieuwland (1993).

In order to guarantee positive conditional volatility we have to restrict the parameters in the estimation procedure. This can lead to some difficulties in the estimation of these models. To ensure positive volatility Nelson (1991) proposed the alternative Exponential GARCH (EGARCH) specification. Here the logarithm of $h_{t}$ is modelled instead of $h_{i}$ per ses. The $\operatorname{EGARCH}(p, q)$ model has the following conditional variance specification, see Andersen (1994):

$l n h_{t}=\alpha_{0}+\sum_{j=1}^{p} \beta_{j} \ln h_{t-j}+\sum_{i=1}^{q} \gamma_{i}\left(\theta \varepsilon_{t-i}+\gamma_{0}\left(\left|\varepsilon_{t-i}\right|-E\left|\varepsilon_{t}\right|\right)\right.$

This specification additionally captures the so-called leverage effect: the asymmetric response of volatility to positive and negative shocks in returns, see Hamilton (1994). If $\gamma_{i}>0$, Nelson's model implies that a deviation of $\| \varepsilon_{i-i} \mid$ from its expected value causes the variance of $\varepsilon_{t}$ to be larger than otherwise, which is in line with the ARCH and GARCH specifications. The leverage parameter $\vartheta$ allows this effect to be symmetric. If $\vartheta \neq 0$, a positive shock has a different effect on volatility than a negative shock in returns of the same magnitude. A number of studies, see for instance Engle and Ng (1993), have shown that there is evidence of a leverage effect: negative shocks seem to increase volatility more than positive shocks. This phenomenon is explained economically as follows: A lower stock price increases the debt to equity relation of a company and this again increases the risk of holding stocks of this company. Asymmetric responses to negative and positive innovations can also be captured by a simple modification of the GARCH specification, see Glosten, Jaganathan and Runkle (1989), who use the following $\operatorname{GARCH}(1,1)$ specification to describe the conditional variance:

$h_{t}=\omega+\alpha_{1} \varepsilon_{t-1}^{2}+\beta_{1} h_{t-1}+\vartheta \varepsilon_{t-1}^{2} S_{t-1}$

Here $S_{t-1}=1$ if $\varepsilon_{t-1} \geq 0$ and $S_{t-1}=0$ if $\varepsilon_{t-1}<0$. If the leverage effect holds, we expect $\vartheta$ to be negative. The non-negativity condition for conditional volatility is satisfied as long as $\beta_{1} \geq 0$ and $\alpha_{1}+\vartheta \geq 0$. In the empirical chapters of this thesis we will estimate several ARCH and GARCH models including those specifications with a leverage effect ${ }^{6}$.

5 This makes numerical optimization methods simpler and allows a more flexible class of dynamic models for the variance.

6 Other related nonlinear (G)ARCH specifications have been proposed, such as Geweke (1986) and Higgins and Bera (1992). 


\subsection{Stochastic Volatility Models}

A competing alternative for the conditional heteroskedastic class of models, where the changing variance is dependent on past squared residuals and past conditional variances, is offered by Stochastic Volatility (SV) models. This alternative way of modelling the time-varying variance is presented in Taylor (1986, 1994) and Harvey (1989) among several others. In SV models the variance is treated as an unobservable variable following a certain kind of linear stochastic process, often of an autoregressive nature. These models have their origin in the continuous time finance literature on several topics, like for instance portfolio diversification, see Merton (1990), and the pricing of options and other derivatives, see for instance Hull and White (1987) and Melino and Turnbull (1990). These diffusion models can be transformed into a discrete time model, see Taylor (1994) and Mahieu (1995), as follows:

$$
\ln P_{t+1}-\ln P_{t}=\gamma+\sigma_{t} \varepsilon_{t+1}
$$

Here $\varepsilon_{t+1}$ is an identically and independently distributed normal random variable with zero mean and unit variance. The logarithmic difference of prices $\left(P_{\mathrm{t}}\right)$ can be interpreted as the returns of the underlying stock. A frequently used specification of the volatility process is offered by an AR(1) process:

$h_{t+1}=\mu+\varphi h_{t}+\eta_{t+1}$

Here $h_{t} \equiv \ln \sigma_{t}$ and the persistence parameter $\varphi$ is restricted to be less than one in order to keep the volatility process to have the usual mean reverting properties. This model has been used in several empirical studies, see for instance Harvey and Shephard (1994) and Mahieu and Schotman (1996), and is mostly referred to as the lognormal model. Note that the error term $\eta_{t+1}$ is identically and independently normally distributed with mean zero and variance $\sigma_{\eta}^{2}$. The correlation between the error terms in both equations is equal to $\rho$. The estimation of these models is very computer intensive. We will discuss various estimation procedures of GARCH and SV models in the empirical chapters.

Taylor (1994) uses an economical framework to motivate temporal changes in the volatility process. He presents an information counting model in which returns are defined by a stochastic number of intra period price revisions, as in Clark (1973), Tauchen and Pitts (1983) and Harris (1986, 1987). Suppose there are $N_{1}$ price changes during a particular trading day $t$, each caused by an unexpected piece of information entering the stock market. Let event $i$ change the daily return $\left(y_{i}\right)$ on day $t$ by $\delta_{i t}$ :

7 It should furthermore be noted that the discrete models in 2.5 and 2.6 converge to continuous time models used in theoretical finance, see Nelson (1990) under which conditions. 
$y_{t}=\omega+\sum_{i=1}^{N_{t}} \delta_{i t} \quad \delta_{i t}-N\left(0, \sigma^{2}\right)$

Clark (1973) and Lamoureux and Lastrapes (1990a) show that when the number of daily information arrivals is large enough, a central limit theorem argument can be made resulting in:

$y_{i} \mid N_{i}-N\left(0, \sigma^{2} N_{t}\right)$

Note that the conditional variable $N_{t}$ is unknown and furthermore that the conditional variance is time-varying because of the random nature of the mixing variable. If we now rewrite 2.10 as:

$y_{t}=\sigma \sqrt{N_{t}} \varepsilon_{t}, \quad \varepsilon_{t} \sim N(0,1)$

and assume that the number of daily information arrivals follows an AR(1) process:

$N_{t}=\beta+\varphi N_{t-1}+\eta_{t}, \quad \eta_{t} \sim N\left(0, \sigma_{\eta}^{2}\right)$

It turns out that this is in fact equivalent to a SV model, see equations 2.5 and 2.6 , in which the volatility is represented by the unobservable and unknown number of information arrivals. Note that the choice of an AR(1) representation for the information variable is important here. Andersen (1996) motivates this choice as follows:

"A couple of considerations are relevant when selecting a dynamic representation for the information variable. First, casual empiricism suggests that news arrivals are positively correlated. When unanticipated news breaks on a given day, more detailed disclosures tend to follow over the next few days or weeks, and different interpretations of the circumstances leading to the event surface. This tends to keep the "story" in the headines for an extended period of time. Moreover, important changes in the tactical orientation of a company do typically play themselves out over longer periods (take-over battles and proxy fights), and these developments are revealed through periodic news updates. Second, and more importantly, judging from the success of modeling return volatility dynamics by means of ARCH processes, and in particular models closely related to the GARCH(1,1), it is clear that an information arrival process governing the dynamic features of return volatility must display a similar type of positive conditional dependency. Andersen (1994) develops a class of stochastic volatility models that are closely related to $\operatorname{GARCH}(1,1)$, termed Stochastic AutoRegressive Volatility (SARV) models. They satisfy the above criteria in that they allow for positive autocorrelation in news arrivals, and they serve as natural stochastic volatility generalizations of relevant ARCH models."

We have to bear in mind that the latent process possibly follows a MA(1) process or even an ARMA $(1,1)$ instead of the assumed AR(1), but in this thesis we 
restrict ourselves to using the process implied by the literature and proposed by Andersen $(1994,1996)$. In various empirical studies the process of information arrival has been proxied by several variables related to the information generating process, like trading volume, see for instance Lamoureux and Lastrapes (1990a) and the number of price changes, see Laux and $\mathrm{Ng}$ (1993). In line with the basic SV model we have to assume here that $N_{t}$ is always positive. Therefore we have to transform the specifications above to a lognormal SV model with an $\mathrm{AR}(1)$ volatility process:

$$
y_{t}=\sigma \varepsilon_{t} \exp \left(\frac{h_{t}}{2}\right), \quad \varepsilon_{t}-N(0,1)
$$

where $h_{1} \equiv \ln N_{i}$, see 2.12 , and therefore it can be interpreted as a volatility or a transformed information arrival process. Estimation of the SV model thus implicitly leads to an estimate of the unobservable information arrival process. In the next section we will show that GARCH and SV models are closely linked to Mixture of Distributions Hypothesis.

\subsection{The Mixture of Distributions Hypothesis}

Stock price changes, when sampled over fixed calendar time intervals, generally appear to be uncorrelated with each other, but the empirical distribution often suffers from substantial leptokurtosis in relation to the normal distribution. A first explanation for this phenomenon was given by Mandelbrot (1963), Fama (1965) and Mandelbrot and Taylor (1967), who advocated the viewpoint that stock price differences are best characterized by a member of the stablle family of distributions with infinite variance. These distributions usually give high values for the measured sample kurtosis, which is in accordance with most empirical studies. Clark (1973) proposed a competing theoretical framework for stock price movements and trading volume by introducing the Mixture of Distributions Hypothesis (MDH), which explores the idlea of using trading volume as a proxy for some unobservable, subordinated stochastic process of information arrival. He started his analysis by stating that the different behaviour of price changes on different days is due to the varying rate of information arrival. Trading days without any relevant informational events are accompanied by few trading activity and therefore a slowly evolving price process. On trading days, where unexpected news leads to changes in the expectations of traders, trading is more lively and the price process evolves much faster. The relation between the price process and the information arrival process is then described by a subordinated stochastic process. 
If we now assume that $T(t)$ denotes a positive stochastic process ${ }^{8}$, representing the varying rate of information arrival, then a new process $X(T(t))$ can be defined. This process is said to be subordinated to $X(t)$. The process of information arrival $T(t)$ is the directing process. Analogously, the distribution of $\Delta X(T(t))$ is subordinate to the distribution of $\Delta X(t)$, where $\Delta X(t)$ displays the role of the evolution, c.q. changes, in the underlying price process, i.e. returns, while $T(t)$ measures the varying rate of information arrival'. $X(T(t))$ is then the price process we actually observe. Clark (1973) shows that, if the intra-daily price increments $\Delta X(t)$ are independent with zero mean and a variance $\sigma^{2}$, then $n$ steps have zero mean and variance $n \sigma^{2}$. This implies that the variance of $\Delta(X(T(t))$ conditional on the rate of information arrival $\Delta(T(t))$ is:

$\operatorname{Var}\left((\Delta X(T(t)) \mid \Delta T(t)=n)=n \sigma^{2}\right)$

In order to be able to test his model and its main distributional implications, Clark (1973) decided to choose trading volume as a proxy for the unobservable speed of information arrival ${ }^{10}$. In Clark's terminology the distribution of $\Delta X(T(t))$ would have a distribution subordinate to that of the intra-daily price increments, and directed by the distribution of daily cumulative trading volume.

The mixture hypothesis has been studied and refined in several studies. Epps and Epps (1976) present a model, in which the variance of the price change of a single transaction is conditional upon the trading volume of this transaction. They assume there is a positive relationship between the extent to which traders disagree when they revise their reservation prices and the absolute value of the price change in the market price. In that context an increase in the disagreement among traders is associated with larger price changes. The relationship between volatility and volume arises then because trading volume is positively related to the extent to which traders disagree when they revise their reservation prices. Their empirical results support Clark's view that the variance of the change in logarithmic price changes depends on trading volume ${ }^{11}$. Westerfield (1977) provides empirical evidence for the subordinate model and finds that absolute price changes vary with the number of transactions during a trading day. The

This process has to be positive as the rate of information arrival cannot be negative.

It should be noted that Clark's (1973) primary goal was to explain why the probability distribution of the daily price changes is leptokurtotic. His opposing hypothesis is tested against alternatives of the stable class of distributions, see Mandelbrot (1963). The variance of Clark's subordinated process is finite for processes having increments with finite variance, and directed by a process with increments of finite mean.

10 Note that this is the result we use in equation 2.10 in section 2.3 .

11 Epps and Epps (1976) however point out that their empirical findings do not necessarily rule out the possibility that the change in logarithmic prices over fixed intervals of time has infinite variance. 
correlation between trading volume and absolute price changes then results because volume is also an increasing function of the number of within-day price changes. The idea of variable transaction times has been extended by Tauchen and Pitts (1983). In their model the correlation between absolute price changes and the volume of trade increases with the variance of the speed of information arrival. They furthermore point out that results can be very misleading, if trading volume is strongly trended, possibly caused by a sharp rise in the number of traders active in the market. The issue of detrending trading volume will be discussed in Chapters 5 through 7.

Based on the ideas of Clark (1973) and Tauchen and Pitts (1983), Harris (1987) presents a simple mixture of distributions model for the joint distribution of daily price changes and daily trading volume. He presents a framework in which he assumes that new unexpected information events result in price changes and trading activity. Harris (1987) argues that investors will revise their portfolios based on this newly entered information. In the resulting conditional mixture model he first assumes that post-event price changes and trading volumes are jointly independently and identically distributed with finite variance. The second assumption is that the number of information events occurring each trading day varies ${ }^{12}$. In his approach $n_{t}$ denotes the number of information events on day $t$. In this setting the total price change and trading volume for a given day $t$ are proportional to $n_{t}$ information events.

This set of assumptions implies that the joint distribution of price changes and trading volumes will be approximately normal conditional on the number of information events. Harris (1986) and Tauchen and Pitts (1983) show that assuming a symmetric distribution for the unexpected information leads to a zero correlation between price changes and trading volume conditional on $n_{4}$. Harris (1987) uses this result, which leads to a conditional covariance of daily price changes with daily trading volume equal to zero ${ }^{13}$. He symbolically represents his mixture model as follow's:

$$
\begin{aligned}
& d p_{t}=N\left(a n_{t}, b n_{t} \mid n_{t}\right) \\
& V_{t}=N\left(c n_{t}, d n_{t} \mid n_{t}\right)
\end{aligned}
$$

$\operatorname{Cov}\left(d p_{t}, V_{t} \mid n_{t}\right)=0$

12 Harris (1987) explicitly notes that this variation can be random, deterministic and seasonal respectively.

13. Under the assumption that investors are rational and use market prices to form their expectations, see Harris (1987) for further details on this subject. 
Here $a, b, c$ and $d$ are all positive parameters and:

$d p_{t}=\sum_{i=1}^{n_{i}} \delta_{i t}$

where $d p$, denotes the total daily price change on day $t$, measured as a logarithmic difference, $n_{t}$ denotes the number of information events on day $t$, whereas $\delta_{i t}$ denotes the $i$-th intra day price change on day $t$. The total price change and volume of trade, $V_{i r}$ for a given day $t$ represent the cumulation of price changes and corresponding volumes that occur as a result of $n_{t}$ new information events. As a consequence, the joint distribution of $d p_{l}$ and $V_{l}$ will be approximately bivariate normal conditional on the speed of information arrival $n_{t}$. In line with the mixture hypothesis, for a given $n$, applying the Central Limit Theorem $(C L T), d p$ is approximately normally distributed with a variance proportional to $n_{i}$. A varying rate of information arrival however implies that the CLT does not hold and therefore the distribution of $d p_{t}$ is subordinate to the distribution of $n_{\mathrm{t}}$.

Harris $(1986,1987)$ performs an indirect cross-security test of the $\mathrm{MDH}$ using both daily and transaction data. His conditional mixture model closely resembles the one earlier presented by Tauchen and Pitts (1983). Subsequently, based on the assumptions mentioned above, Harris (1987) presents a set of predictions with respect to the implied distributional patterns of daily price changes, daily volume of trade and the daily number of transactions. In addition, he presents some implications the MDH has for transaction data. At first Harris (1987) presents a number of predictions with respect to the main distributional features of price changes and trading volumes. He predicts that the marginal unconditional distributions of $d p_{t}$ and $V_{t}$ are skewed substantially, where price skewness is expected to be smaller than volume skewness. The frequently observed kurtosis in empirical investigations on daily price changes stems from the fact that, as a consequence of the mixing process documented before, the conditional variance of the price changes is proportional to the mixing variable $n_{i}$. The unconditional distribution therefore has fat tails and is possibly spiked, because it is an average of diffuse and compact densities, due to the variation in the rate of information arrival. In this setup price and volume skewness are a result of the fact that both the mean and variance of their conditional distribution are proportional to the mixing variable. Trading volume is expected to be right-skewed because its conditional density is an average of spiked densities near zero, in the case of a slowly evolving rate of information arrival, and diffuse densities for a high information rate centred far from zero. Price skewness is smaller because for price changes the conditional mean is small relative to its conditional variance.

The second set of predictions made by Harris (1987) concerns the correlation between price changes and trading volume. Based on the mixture model, he 
expects squared daily price changes to be positively correlated with trading volume due to the fact that, in line with the first set of predictions, the mean and variance of the conditional distributions of both variables are proportional to the same mixing variable. Both price changes, squared price changes and trading volume are likely to have relatively large values when the information process evolves quickly and consequently small numbers when the information process is slower. This results in a positive correlation between these variables, see also Harris (1986).

Harris (1987) then gives a number of predictions regarding the time series behaviour of price changes and trading volume. He argues that the variance of the price changes will not be stationary if the number of information events varies through time ${ }^{14}$. This follows directly from the above mentioned proportionality in the model setting. Furthermore he states that the time series of all variables are autocorrelated if there is evidence of autocorrelation in the mixing variable. In line with the second set of predictions this autocorrelation will be strongest for the volume series and weakest for the price changes series ${ }^{15}$. Assuming that transactions occur at a uniform rate in time, i.e. transactions directly follow the information event, Harris (1987) presents a fourth set of predictions based on his mixture model. Then, in a market place where the number of traders is fixed, the number of daily transactions, $N_{1}$, is proportional to the number of information events in time. This proportionality yields the prediction that the number of transactions is correlated in decreasing order with trading volume, squared price changes and price changes respectively ${ }^{16}$. As the variation in the number of transactions is directly linked to the variation of the mixing variable, we expect $N_{1}$ to have the same autocorrelation as $n_{1}$. If this model setting is correct this implies that the daily number of transactions is a good proxy for the time-varying information evolution rate. We will test this part of the $\mathrm{MDH}$ in the empirical chapters of the thesis.

The final set of predictions concerns the distribution of adjusted daily price changes, defined by:

$$
A_{t}=\frac{d p_{t}}{\sqrt{N_{t}}} \sim N\left(a \frac{1}{\sqrt{k}} \sqrt{n_{t}}, \frac{b}{k}\right)
$$

14 This non-stationarity in the variance was in fact the starting point of the discussion on the link between return volatility and trading volume, see Lamoureux and Lastrapes (1990a). As a result of the fact that the fraction of variation due to the varying character of the speed of information arrival is largest for the price change series.

16 Caused by the proportionality of the conditional mean of all variables to the same mixing variable. 
where $A$ denotes the adjusted return series created by dividing daily price changes by the square root of the daily number of transactions; $k$ denotes the constant of proportionality between $N_{t}$ and $n_{i}$. This exercise is quite interesting as daily variation due to $n_{i}$ in the conditional variance of price changes is eliminated. Hence, daily variation in the conditional mean will be smaller. This measure is expected to be more normally distributed than the unadjusted series and less affected by the mixing variable. In particular, it should be less kurtotic and skewed than the unadjusted price changes. Empirical results on a sample of stocks listed on the New York Stock Exchange (NYSE) largely supported the predictions made by Harris (1987). More specifically, a direct implication of this research is that the daily number of transactions in a given stock may be a good estimate of the varying rate of information arrival. Adjusting daily data by dividing price changes through the daily number of transactions leads to a rejection of the mixture hypothesis, which confirms that the wnobservable information evolution rate is related to the transaction generating process. Richardson and Smith (1994) conduct a direct test of the mixture model. They find some evidence against the MDH. Moreover, they implicitly document several stylized facts of the unobservable information flow. In the next section we will discuss the empirical studies based on or related to the $\mathrm{MDH}$.

\subsection{Empirical Tests of the MDH}

Tauchen and Pitts (1983) present a bivariate mixture model in which both daily price changes and daily trading volumes are endogenous variables, simultaneously directed by the information arrival process. They explicitly assume that the unobservable mixing variable is a random variable and therefore the unconditional distribution of price changes and volume is a mixture of independent bivariate normals, where the information flow is the mixing variable. It should however be noted that Tauchen and Pitts (1983) assume that the mixing variable is serially independent, whereas a number of other studies allow for a positive serial dependence in the mixing variable, see for instance Lamoureux and Lastrapes (1990a) ${ }^{17}$.

Among empirically oriented econometricians, Lamoureux and Lastrapes (1990a) (hereafter: LL90) broadened the discussion on the relationship between trading volume and stock return volatility by their statement that the time-varying features of the rate of information arrival possibly explain heteroskedasticity in stock returns. Using a typical univariate GARCH framework, they showed that

17 We will estimate a bivariate mixture model equivalent to the Tauchen and Pitts (1983) specification in Chapter 6 using a sample of intra-daily data. The mixing variable is modelled both static (serially independent) and dynamic (serially dependent). 
including contemporaneous trading volume as a weakly exogenous variable leads to lower persistence in conditional volatility. In this setup trading volume is explicitly used as a proxy for the unobservable rate of information arrival, largely inspired by the $\mathrm{MDH}$ and Clark (1973). Theoretically, assuming serial correlation in the speed of information, they show that this implies an autoregressive structure in the conditional variance, similar to the GARCH specification as proposed by Bollerslev (1987). LL90 use this as a motivation to explain GARCH in USA stock return data.

They were however criticized in the financial debate on this issue, see for instance Bollerslev, Chou and Kroner (1992). Their theory was based on the link between the mixture of distributions hypothesis and GARCH models. LL90 made the relatively strong assumption that trading volume is a suitable proxy for the unobservable information evolution rate. Market microstructure models, see for instance Kyle (1985), show that trading volume is possibly not just induced by new information entering the stock market. Liquidity motives may also explain a large part of the observed trading volume in the stock market ${ }^{18}$. Furthermore, the inclusion of contemporaneous trading volume possibly leads to simultaneity bias in the estimation procedure. According to the bivariate model for price changes and trading volume of Harris (1987), which seems plausible, the volatility of price changes and trading volume are determined by the unobservable information flow simultaneously. "Therefore, the results in LL90 could be subjected to a possible simultaneity bias ${ }^{19}$. It should however be noted that their study was extremely valuable as it was an often quoted starting point for several research projects into the relationship between volatility and trading volume on the one and a possible explanation of the GARCH phenomenon on the other hand.

An alternative univariate approach to provide the lacking economic motivation for GARCH in stock returns is offered by Laux and $\mathrm{Ng}$ (1993). They provide more direct evidence on the mixture of distributions hypothesis, using it as a possible economic motivation for GARCH. Their methodology is different to LL90 in several respects. A common feature in the financial literature is to decompose the volatility process into systematic and unique parts, e.g. in the Capital Asset Pricing Model (CAPM). Laux and Ng (1993) use this as an additional tool to explain GARCH. Furthermore they propose an alternative proxy for the rate of information arrival: the number of observed price changes per period. In their opinion this measure is more inclusive than trading volume because it even reflects information arrival that does not directly result in trading volume. Another advantage of choosing this proxy is that it is not as sensi-

18 Think for instance of large institutional investors who mainly trade for liquidity reasons.

19 Note that an inclusion of lagged volumes did not lower the persistence in volatiluty in LL90. 
tive to the precise definition of the scope of the market, especially because Laux and Ng (1993) use foreign exchange futures data. Price changes occur in all important financial markets in response to the arrival of new information, if at least the arbitrage mechanism is effective. Moreover, in contrast to LL90, Laux and $\mathrm{Ng}$ (1993) use intra-daily data, which has the advantage that we should be less concerned about the previously mentioned simultaneity bias. In brief time intervals, the reaction of investors to a new piece of information will probably take place in a later time period. In addition, it seems reasonable to assume that investors will only change their portfolios after price changes are sufficient to justify the corresponding transaction costs. The main result of their empirical analysis is that simple univariate GARCH models of foreign exchange futures are misspecified due to their failure to account for the fact that systematic and unsystematic volatilities have different persistence levels. Locke and Sayers (1993) try to explain the persistence in the variance structure of intra-daily S\&P 500 index future series by incorporating various proxy variables ${ }^{20}$. The proxies were contract volume, floor transactions, the number of price changes, executed order imbalance and a composite variable respectively. All proxies were able to explain a large part of the return variance. Correcting for the speed of information arrival by using the proxies did however not remove all serial dependence in the second moment.

Lamoureux and Lastrapes (1994) (hereafter LL94) responded to the before mentioned criticism by presenting a new version of their mixture model in which the assumption that trading volume is weakly exogenous is relaxed. In their specification, volume is endogenized in the model. A latent common factor restricts the joint density of volume and returns. In order to circumvent the computational burdensome maximum likelihood estimation technique, they employ a signal-extraction strategy. For each observation they estimate the values of the latent information process. Then the return series are adjusted by the estimated latent variable series. They find however that correcting for the serial dependence in the information arrival process does not eliminate GARCH persistence in variance, which is consistent with the findings of Locke and Sayers (1993). This implies that the mixture of distributions hypothesis does not fully explain the observed clusters in volatility.

In another direct test of the MDH, Jones, Kaul and Lipson (1994) argue that the positive relationship between volatility and trading volume may be the result of the positive relationship between volatility and the daily number of transactions. They find that average trade size (i.e. volume) has no information beyond that contained in the frequency of transactions. Therefore it seems appropriate

20 Locke and Sayers (1993) had the advantage to be able to use minute by minute data. It is therefore hardly possible to compare their results with those of LL90. 
to modify certain features of the standard MDH, see Andersen (1996) for a first but excellent approach. Richardson and Smith (1994) also perform a direct test of the mixture hypothesis. They find evidence against the MDH. In particular, they argue that volume may not be conditionally normal as in equation 2.16 , as proposed by Harris (1987). In that case linking price changes and trading volume to the same mixing variable via a bivariate conditional normal model specification may not be the correct specification. Their empirical evidence implies that volume should be skewed to the right conditional on the information flow. Possible candidates for the distribution of volume are then a lognormal distribution or a Poisson distribution. Whether this would significantly improve the fit is an empirical question. We try to answer this question in the empirical part (Chapter 7) by estimating a mixture model with a Poisson distribution for trading volume.

A branch of the literature only indirectly linked to the MDH is offered by Gallant, Rossi and Tauchen (1992) who apply a semi-nonparametric (SNP) estimation technique to daily data on the returns of the S\&P 500 Composite Stock Index and trading volume on the New York Stock Exchange ${ }^{21}$. Their extensive empirical analysis renders several consistent patterns: the existence of a positive correlation between conditional volatility and trading volume. Furthermore they find that large price shocks are followed by high trading volume. A possible explanation for this could be that high shocks in returns lead to uncertainty, which increases the probability of disagreement among traders. "This could result in more trading action, see for instance Epps and Epps (1976). Conditioning on lagged trading volume substantially weakens the leverage effect ${ }^{22}$. Finally, they show that conditioning on lagged volume yields a positive risk-return relationship. Another example of this literature is a study of Tauchen, Zhang and Liu (1993), who use another nonparametric - and nonlinear - estimation procedure. They find substantial evidence across their sample of stocks that trading volume responds non-linearly to price shocks and in such a way that it could not be detected using standard symmetric parametric models. The number of shares traded increases, independent of the sign of the shock. In the next section we will briefly discuss the market microstructure literature which is important for the understanding of the more empirically oriented modified mixture model of Andersen (1996).

21 In this thesis we mainly discuss those studies where the relationship between volatility and volume is placed in a context linked to the MDH.

22 For a detailed explanation of the leverage effect, see Hamilton (1994). 


\title{
2.6. The Market Microstructure Literature
}

Parallel to the developments in the mixture of distributions theory, a whole new area of research emerged in the last two decades: the market microstructure literature ${ }^{23}$. This branch of the literature suggests that several exponents of stock markets are related to the return volatility process, see OHara (1995) for an excellent overview of the most important publications. These factors are the number of transactions, trading polume, the bid-ask spread or the liquidity of the stock market. In this thesis we explicitly highlight the role of trading volume in understanding the behaviour of prices and volatility in stock markets. Karpoff (1987) provides an elaborate study on the empirical link between price movements and trading volume. The outcome of the bulk of price-volume studies is that there is a strong link between absolute price changes and trading volume. These empirical facts can not easily be explained in a theoretical framework. O'Hara (1995) however stresses the obvious relevance of volume:

\begin{abstract}
"One reason it is difficult to evaluate the link of price and volume is that it is not obvious what information volume, in itself, provides to the market. Just as traders can learn by watching prices, it seems likely they could learn by watching volume. In the extreme case, it is possible that volune alone could reveal whderlying information, with prices playing a redundant information role. A more likely scenario is that the conbination of price and tolume conld protide information to the market in much the same way that the sequence of prices reveraled information, ...."
\end{abstract}

OHara (1995) discerns two classes of market microstructure models. The first is mainly represented by the work of Demsetz (1968), who was one of the first researchers that gave attention to the underlying micro-foundations of security markets. This led most of the early studies in the direction of explaining and understanding the behaviour of agents and institutions on these markets. This class of models, called inventory models, was further developed by giving attention to the way market prices arise, given the nature of the order flow and the market mechanism. All of these approaches, see Garman (1976), Stoll (1978) and Cohen, Maier, Schwartz and Whitcomb (1981), emphasize the uncertainty in the order flow, which possibly results in inventory problems for the specialist or dealers and execution problems for the trader. A very important implication of inventory models is that transaction costs determine the bid-ask spread.

The other class of models proposed by O'Hara (1995), is represented by the information-based models. The assumption of asymmetric information, see

${ }^{23}$ It is an almost impossible task to give a brief overview of the market microstructure literature. We chose to cite those studies in subsequent chapters that are most relevant for our empirical research objectives. 
Glosten and Milgrom (1985) and Easly and O'Hara (1987), leads to the proposition that the nature of the order flow is not exogenous to the dealer's problem, and therefore the trade itself contains information ${ }^{24}$. Glosten and Milgrom (1985) use this insight in their model of the market maker's pricing decision. In particular it is assumed that, in competitive markets, informed agent's trades will reflect the information content as follows: informed investors sell in the case of bad news and buy in the case of good news. This implies that if the market maker receives a sell order of a trader, this could signal that this particular trader knows bad news. On the other hand it could mean that the trader is simply uninformed. The market maker does not know which of both scenarios is the case and therefore he adjusts his beliefs conditional on the type of trade that occurs. Glosten and Milgrom (1985) show in this model setting that the market maker eventually learns the informed trader's information. Their study is in fact an example of the changing focus of the market microstructure literature at that point in time. The central point of attention became analysing how the market maker learns from the order flow and how this affects the movement of prices and trading volume over time.

Subsequently, the information-based models were further developed by assuming that informed traders may have an incentive to act strategically to maximize their profits, see Kyle (1985). In this strategic model environment informed investors are allowed to time their trades or to choose their trade size. This can lead to a much different equilibrium when compared to the information-based models without strategic behaviour. An important feature of this type of models is that information may be incorporated into prices through time, given the strategic behaviour of an informed agent. An important drawback of the strategic models so far is that they assume that uninformed traders are not permitted to act strategically. This issue is addressed in several publications. The main representatives of this type of models are Admati and Pfleiderer $(1988,1989)$ and Foster and Viswanathan (e.g. 1990). Allowing uninformed traders to time their trades implies that uninformed investors could also induce price and volume patterns ${ }^{25}$. The information asymmetry in the class of information-based models induces trading activity, where the size and quantity of the trades reflect the disagreement among the market players. As a result there is a positive relation between volume and absolute price changes. An alternative classification of theoretical market microstructure models is given by Jones, Kaul and Lipson (1994), who divide the approaches into two subcategories: competitive

24 With respect to the bid-ask spread, a study of Copeland and Galai (1983) shows that information alone is sufficient to induce market spreads.

25 The majority of these studies concentrates on intra-daily dependencies and patterns in (squared) returns and trading volume, see for instance Admati and Pfleiderer (1989). Predictions for inter-day patterns are rarely made. 
and strategic models. In competitive models with an asymmetric information structure, see for instance Pfleiderer (1984) and Holthausen and Verrecchia (1990), the trading activity is positively related to the quality of the information obtained by informed trader $s^{26}$. In strategic models differences in the information structure also lead to trading, but an insider may chose to divide his trading action over a few trading days, so that he can not be "detected" as an informed trader, see for instance Kyle (1985) among others ${ }^{27}$.

Finally, Andersen (1996) combines the general results of the market microstructure theory with the MDH and the framework of stochastic volatility models. He explicitly makes a difference between trading volume induced by liquidity trading and trading volume induced by informed traders. The resulting Modified Mixture Model (MMM) is largely inspired by the before mentioned market microstructure model of Glosten and Milgrom (1985). Another interesting feature of this model is that it incorporates the concept of Stochastic Volatility, thereby assuming a dynamic structure for the latent, and possibly serially correlated, information process. The modified mixture model will be estimated in Chapter 7 .

\subsection{Concluding Comments}

In this chapter we introduced the discussion on the link between two important factors in the (dis-)equilibrium dynamics of stock markets: the return volatility and trading volume. The relationship between both variables is important in several aspects of financial economics. Throughout this thesis we mainly focus on the empirical investigation of this relationship, thereby using the insights of the MDH literature. This hypothesis states that the return volatility process and the dynamic process that generates trading volume are heavily linked together by the same latent information process. This has certain implications for the empirical distribution of both returns, volatility and trading volume. The MDH has been tested in several empirical studies. The results are mixed: some studies find weak evidence against the mixture hypothesis others confirm the predictions of the $\mathrm{MDH}$.

In the empirical chapters we present several models originated from or linked to the standard $\mathrm{MDH}$. Both univariate and bivariate model specifications are

26 It should be noted that Harris and Raviv (1993) show that even in a competitive market, with a perfectly symmetric information structure, there would be a positive relation between trading volume and volatility.

27 Holden and Subrahmaryam (1992) however show that trading volume is positively related to the quality of information in both groups of models. 
discussed. If we are to study the relationship between volatility and volume we however first have to specify the volatility process. We introduced two classes of models: conditional heteroskedastic models (GARCH) and Stochastic Volatility (SV) models. Both classes of models will serve as a tool to investigate the link between volatility and trading volume on stock markets. The more theoretically oriented market microstructure literature will be addressed in Chapter 7 , where we combine the standard MDH with a theoretical market microstructure model with an asymmetric information structure. A second but less important point of interest throughout this thesis is the choice of the estimation method. We will apply several different techniques: (Quasi) Maximum Likelihood Estimation, Simulated Maximum Likelihood Estimation and Bayesian Analysis using Markov Chain Monte Carlo. Although the choice of the estimation method is no primary goal, we obtain interesting results in the empirical section. 


\section{CHAPTER 3}

\section{German Stock Market Dynamics}

\subsection{Introduction}

The debate regarding the validity of empirical asset return distributions continues to be an issue of central concern in the financial economics literature, see for instance Bollerslev, Chou and Kroner (1992). These empirical distributional properties have been tested for stock indices returns by Baillie and DeGennaro (1990), French, Schwert and Stambaugh (1987), Chou (1988), Akgiray (1989), Jorion (1988), Nelson (1989, 1991) and Nieuwland (1993) among others. Probably the most important factor that has generated this considerable interest, is the fact that these distributional properties have a direct impact on the validity of theoretical models in financial economics. Many standard asset pricing models imply the martingale difference model in which price changes are uncorrelated and hence unpredictable in the mean. Furthermore, the Black and Scholes (1973) option pricing model assumes a continuous time stochastic process for the representation of the price behaviour in the form of a geometric Brownian motion where the log of the price relatives are assumed to be independent and identically normally distributed. Also, the assumptions on the distributional properties of the price process are critical in many tests of the efficient market hypothesis.

As is well documented in the literature, empirical distributions of asset returns exhibit leptokurtic behaviour and clusters of high and low volatility. The AutoRegressive Conditional Heteroskedasticity (ARCH) class of models, introduced by Engle (1982) and generalized (GARCH) by Bollerslev (1986), have been shown to provide a good fit for many financial return time series ${ }^{1}$. GARCH. imposes an autoregressive structure on the conditional variance, parametrized as a linear function of past squared innovations and lagged conditional variances, allowing volatility shocks to persist over time. This persistence captures the propensity of large absolute returns to cluster in time. Moreover it can explain the well documented non-normality and non-stability of empirical asset return distributions, see especially the pioneering work of Mandelbrot (1963)

1 See e.g. Bollerslev (1987), Lamoureux and Lastrapes (1990), Baillie and Bollerslev (1989). 
and Fama (1965). Studies by Baillie and Bollerslev (1989), Boothe and Glassman (1987), Hsieh (1989), Jorion (1988), Meese and Rogoff (1983) and Wolff (1987) provide extensive statistical evidence on US Dollar exchange rates and US stock market returns. Overall, the findings overwhelmingly favour the conclusion that the assumption of conditional normality does not capture all of the excess kurtosis observed in both high frequency stock and exchange rate returns. Several alternative conditional distributions have consequently been employed in the literature, for instance the student $t$-, normal Poisson, generalized error and normal-lognormal distributions, see Bollerslev, Chou and Kroner (1992) and Taylor (1994). Interestingly, this branch of the literature pays relatively little attention to observed statistical distributions of European asset returns. In this chapter we aim to provide extensive statistical evidence for German stock market indices. The observed leptokurtosis may be explained by several classes of models. Therefore, alternative time series processes and distributional specifications characterizing the German stock market are considered. We estimate GARCH models and GARCH-in-mean models for three German stock market indices, using weekly and monthly data over the 1973-1992 period. The GARCH-in-mean model, developed by Engle, Lilien and Robbins (1987) which we propose can be used in addressing questions regarding the risk-return tradeoff in a time series context where the conditional variance may be time varying. In addition to exploring normal conditional densities, the student $t$-distribution is employed. The research will form a body of evidence which can serve as a frame of reference for further research.

The main purpose of this chapter is to introduce the class of GARCH models and its empirical implications. We will estimate several univariate models which will serve as a benchmark in later chapters. The remainder is organized as follows. In section 3.2 we describe our data set and its summary statistics. The methodology and models employed to describe the patterns followed by German stock market indices returns are explained in section 3.3. Section 3.4 presents the main empirical results of this chapter. Section 3.5 contains our concluding comments and an outlook to the empirical objectives of Chapter 4 , where we introduce a multiplicative GARCH model for volatility and volume.

\section{3:2 Data and Summary Statistics}

Our data base contains three weekly and monthly German stock market indices. One of the indices used is the Datastream Total Market Index (DS-index DL), which contains all stocks quoted on the Frankfurt stock exchange. In addition, two alternative German stock market indices were included, the Frankfurter Allgemeine Zeitung (FAZ) index and the Commerzbank (COMM) index. The indices are value weighted and adjusted for stock dividends, capital modifica- 
tions and unusually large dividend payments. The data were obtained from Datastream, a UK incorporated data service company. Even though daily stock indices returns are available, we choose to employ weekly and monthly data in order to avoid issues surrounding the day-of-the-week effect with regard to stock return volatility (on which, see Hsieh (1989), for example). Our sample includes 1003 weekly and 231 monthly observations, ranging from 12th. January 1973 through 25th. March 1992.

In Tables 3.1 and 3.2 we present summary statistics for respectively weekly and monthly stock indices returns. Continuously compounded returns are used, defined as the difference in logarithmic value of the two consecutive observations. For the period analysed all stock returns are subject to substantial skewness and kurtosis. Under the normality hypothesis the corresponding measures would have asymptotic distributions of $N(0,6 / T)$ and $N(3,24 / T)$ respectively. Furthermore, to assess the distributional properties of stock index returns, the Bera Jarque (1982) normality test and the Kiefer Salmon (1983) Lagrange multiplier normality test are reported in the tables, where the latter represents a joint test using both skewness and kurtosis and the latter being a LM test for normal skewness (KS-1) and normal kurtosis (KS-2), respectively. Overall, the evidence presented suggests a consistent rejection of the normality hypothesis. Thus, in spite of the notion that leptokurtic unconditional densities of ARCH processes approach normality by temporal aggregation, see Diebold (1988) and Baillie and Bollerslev (1989), it appears that the monthly series used here may be characterized as highly leptokurtic.

In addition, Tables 3.1 and 3.2 report the Ljung-Box (LB) (1978) and Diebold (1988) test statistics for $k$-th order serial correlation in stock returns per se, absolute returns and squared returns respectively. As the choice of the appropriate lag length is somewhat arbitrary, both $\mathrm{LB}(50)$ and $\mathrm{LB}(25)$ were calculated. Diebold (1988) proposes an adjusted Ljung-Box test statistic to alllow for heteroskedasticity. Diebold (1988) showed that in the presence of ARCH effects, the Ljung-Box statistic has a larger empirical size than a nominal test size of $5 \%$ because the asymptotic variance of the autocorrelations under ARCH is larger than the null of Gaussian white noise. Diebold (1988) therefore proposed a for conditional heteroskedasticity adjusted Ljung-Box statistic which preserves the proper size.

2 The weekly quotes are Wednesday closing prices. Wednesdays were chosen because very few holidays occur on that day, and there is no problem of "weekend effects". When the quotes fall on a holiday or weekend (monthly quotes), the next business day is chosen.

3 Here $T$ is equal to the number of observations. 
Table 3.1 Summary Statistics of Weekly Returns $(12 / 1 / 73-25 / 3 / 92)$

\begin{tabular}{|c|c|c|c|}
\hline & Comm-index & FAZ-index & DS-index $D L$ \\
\hline Mean & 0.001 & 0.001 & 0.001 \\
\hline St. Dey. & 0.022 & 0.022 & 0.020 \\
\hline Skewness & $-0.840^{\circ:}$ & $-0.940^{\circ}$ & $-1.049^{\circ}$ \\
\hline Kurtosis & $8.611^{1.4}$ & $8.663^{\circ}$ & $9.344^{\prime \prime}$ \\
\hline Blitest & $1430.80^{* 1 *}$ & $1485.00^{\circ}$ & $1862.00^{* *}$ \\
\hline$\times 5-1$ & $117.57^{* *}$ & $147.37^{\circ}$ & $183.57^{* *}$ \\
\hline$k 5-2$ & $1313.20^{\circ}$ & $1337.61^{*}$ & $1678.41^{\circ}$ \\
\hline $\mathrm{LB}(50): R$ & $72.22^{*}$ & $74.64^{\prime \prime}$ & $88.22^{* *}$ \\
\hline$D(50) R$ & 44.94 & 44.70 & 47.53 \\
\hline$\angle B(50)$ IR & $648.03^{* *}$ & $60455^{* *}$ & $621.79^{\circ}$ \\
\hline $\operatorname{LB}(50) R^{2}$ & $285.67^{: n x}$ & $31097^{* *}$ & $332.57^{*}$ \\
\hline$L B(25) R$ & 28.47 & $47.11^{\prime \prime \prime}$ & $60.40^{\circ *}$ \\
\hline$D(25) R$ & 11.03 & 24.47 & 27.03 \\
\hline$L B(25) \| R$ & $213.09^{\circ}$ & $445.30^{* * *}$ & $460.02^{\circ \prime}$ \\
\hline $\operatorname{LB}(25) \mathbb{R}^{2}$ & $176.44^{\circ}$ & $241.87^{9 x}$ & $267.12^{\circ}$ \\
\hline Autocor $(1)$ & $\begin{array}{c}0.098 \\
(0.054)\end{array}$ & $\begin{array}{c}0.115 \\
(0.055)\end{array}$ & $\begin{array}{c}0.146 \\
(0.057)\end{array}$ \\
\hline Autocor (2) & $\begin{array}{c}0.111 \\
(0.057)\end{array}$ & $\begin{array}{c}0.109 \\
(0.058)\end{array}$ & $\begin{array}{c}0.131 \\
(0.062)\end{array}$ \\
\hline Autocor (3) & $\begin{array}{c}0.044 \\
(0.045)\end{array}$ & $\begin{array}{c}0.048 \\
(0.045)\end{array}$ & $\begin{array}{c}0.045 \\
(0.048)\end{array}$ \\
\hline Autocor (4) & $\begin{array}{l}-0.023 \\
(0.039)\end{array}$ & $\begin{array}{l}-0.013 \\
(0.039)\end{array}$ & $\begin{array}{l}-0.005 \\
(0.038)\end{array}$ \\
\hline Autocor (5) & $\begin{array}{l}-0.026 \\
(0.040)\end{array}$ & $\begin{array}{l}-0.035 \\
(0.041)\end{array}$ & $\begin{array}{l}-0.034 \\
(0.040)\end{array}$ \\
\hline
\end{tabular}

"denotes statistical significance at the $5 \%$ level, " denotes statisticall significance at the $1 \%$ level, whereas BJ-test gives the Bera-Jarque test for normality and KS-1 and KS-2 denote the Kiefer-Salmon Normality test for skewness and kurtosis. LB ( $p$ ) denotes the Ljung-Box statistic for serial correlation using $p$ lags. Autocor $(p)$ denotes the $p$-th order autocorrelation; robust standard errors are given in parentheses.

Any evidence on serial correlation in German stock returns using the standard Ljung-Box test vanishes, when allowing for conditional heteroskedasticity. In contrast to the monthly stock returns, weekly stock index returns exhibit substantial first (and second) order autocorrelation. The absolute and squared weekly stock returns are much more autocorrelated than the raw returns, which is indicative of a strong nonlinear dependence (conditional heteroskedasticity) and the aforementioned clustering phenomenon. One notes that for monthly stock index returns, only significant serial autocorrelations are presented in the absolute returns series, which could be indicative of no conditional heteroskedasticity. In Figure 3.1 we provide graphs of weekly prices, returns, absolute returns and squared returns respectively. These graphs indicate substantial variation in the FAZ index series. Note that this variation is significantly smaller for the period 1973-1983 than for the period 1982-1992.

4 The choice of the index to graph was made arbitrarily. Results are the same for the other indices. 
Table 3.2 Summary Statistics of Monthly Returns $(12 / 1 / 73-25 / 3 / 92)$

\begin{tabular}{|c|c|c|c|}
\hline & Comm-index & FAZ-index & DS-index DL \\
\hline Mean: & 0.004 & 0.005 & 0.005 \\
\hline St. Dev. & 0.050 & 0.048 & 0.047 \\
\hline Skewness & $-1.469^{\circ}$ & $-1.646^{3.4}$ & $-1.664^{\circ 6}$ \\
\hline Kurtosis. & $12.379^{* *}$ & $13.316^{4 *}$ & $14.020^{\prime \prime *}$ \\
\hline Bjutest & $921.65^{\circ *}$ & $1118.90^{\circ \circ}$ & $1265.11^{\circ *}$ \\
\hline KS-1 & $82.38^{* 0}$ & $103.46^{* *}$ & $10573^{\circ *}$ \\
\hline $\mathrm{KS}-2$ & $839.27^{* *}$ & $1015.40^{\circ}$ & $1159.38 *$ \\
\hline $\operatorname{LB}(50) R$ & 54.17 & 56.78 & 56.38 \\
\hline$D(50) R$ & 48.21 & 51.04 & 52.72 \\
\hline$L B(50) \mid R \|$ & $77.09^{\circ *}$ & $77.82^{n+1}$ & $83.50^{* 4}$ \\
\hline$L B(50) R^{2}$ & 24.83 & 20.25 & 19.04 \\
\hline $\mathrm{LB}(25) \mathrm{R}$ & 27.22 & 30.02 & 29.42 \\
\hline$D(25) R$ & 25.66 & 28.37 & 28.11 \\
\hline$L B(25)\|R\|$ & $43.19^{\circ}$ & $40.33^{\prime \prime}$ & $45.27^{\circ}$ \\
\hline $\operatorname{LB}(25) R^{2}$ & 14.99 & 10.01 & 10.80 \\
\hline \multirow[t]{2}{*}{ Autocor (1) } & 0.084 & 0.097 & 0.109 \\
\hline & $(0.068)$ & $(0.069)$ & $(0.068)$ \\
\hline \multirow[t]{2}{*}{ Autocor (2) } & 0.055 & 0.055 & 0.052 \\
\hline & $(0.065)$ & $(0.063)$ & $(0.064)$ \\
\hline \multirow[t]{2}{*}{ Autocor (3) } & 0.043 & 0.057 & 0.046 \\
\hline & $(0.073)$ & $(0.079)$ & $(0.077)$ \\
\hline \multirow[t]{2}{*}{ Autocor (4) } & -0.041 & -0.025 & -0.025 \\
\hline & $(0.077)$ & $(0.074)$ & $(0.077)$ \\
\hline \multirow[t]{2}{*}{ Autocor (5) } & -0.022 & -0.040 & -0.036 \\
\hline & $(0.058)$ & $(0.057)$ & $(0.059)$ \\
\hline
\end{tabular}

"denotes statistical significance at the $5 \%$ level, " denotes statisticall significance at the $1 \%$ lewel, whereas BJ-test gives the Bera-larque test for normality and KS-1 and KS-2 denote the Kiefer-Salmon normality test for skewness and kurtosis. LB (p) denotes the Ljung-Box statistic for serial correlation using $p$ lags. Autocor (p) denotes the $p$-th order autocorrelation; robust standard errors are given in parentheses.

The shifts of the FAZ index series due to both stock market crashes in the eighties can be clearly detected in these figures. The graph of the return series clearly shows that volatility comes in waves. That is, large (small) changes in share prices tend to be followed by large (small) changes and this phenomenon is more marked for higher frequency series. The absolute and squared return series confirm this. In order to remove possible first order autocorrelation and to differentiate between correlation effects and heteroskedasticity effects, the following OLS regression was fitted:

$R_{t}=\varphi_{0}+\varphi_{1} R_{t-1}+\varepsilon_{t}$ 

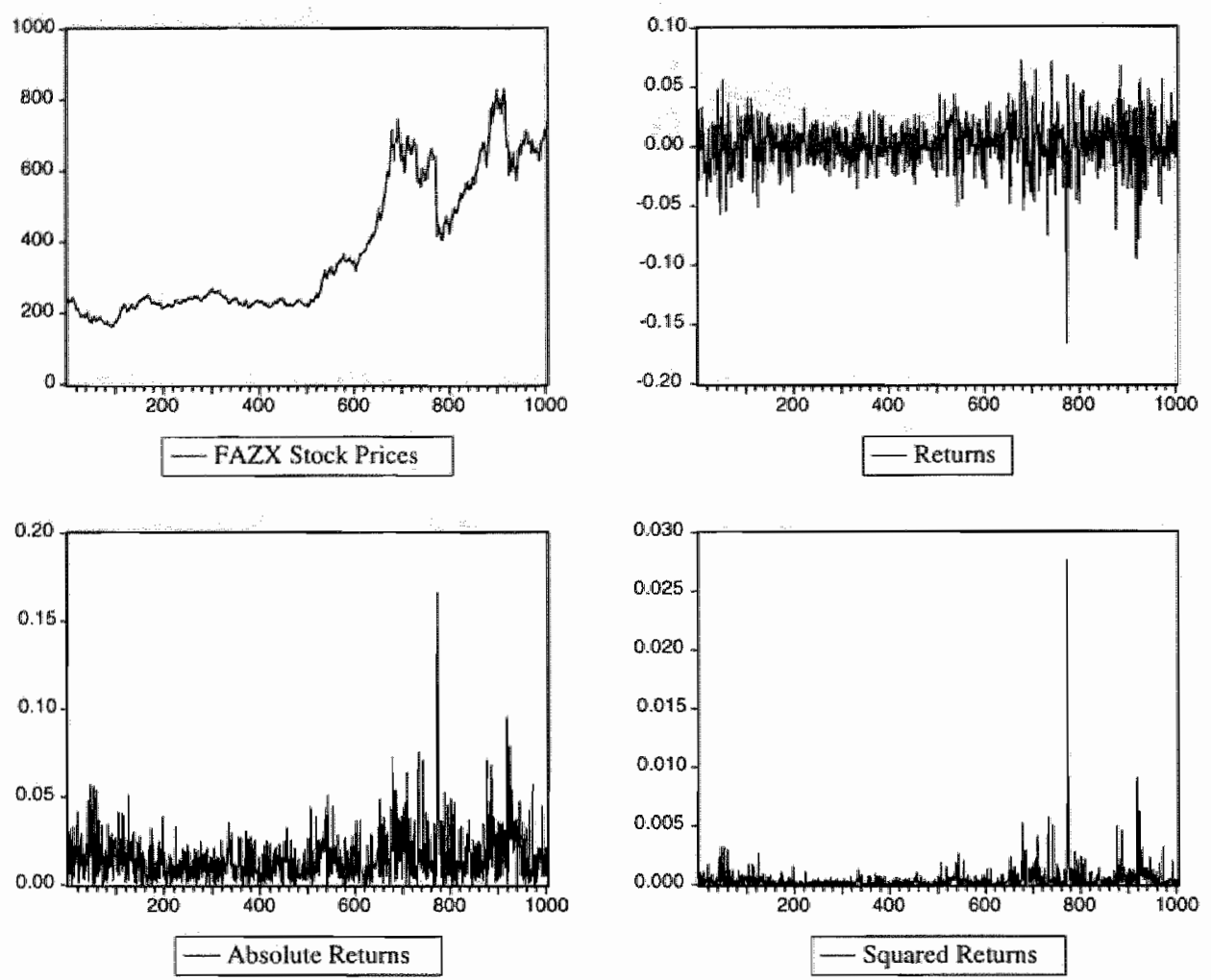

Figure 3.1 The FAZ index: Weekly Prices, Returns, Absolute Returns and Squared Returns.

We employ two different approaches to test for the presence of heteroskedasticity in German stock returns. First, the Lagrange multiplier tests for autoregressive conditional heteroskedasticity, see Breusch and Pagan (1979), are performed. The second test we apply is a nomparametric test based on finitestate homogeneous Markov chains, see Gregory (1989) ${ }^{5}$. Using Monte Carlo analysis Gregory (1989) concludes that under other distributions than the normal the LM test is biased towards the null hypothesis of no ARCH, and that the Markov Chain test is superior to the LM test in terms of better finite sample properties. Both tests only require estimation under the null hypothesis of no heteroskedasticity and are appropriate under all distributional assumptions ${ }^{6}$.

5 For a more detailed description on this test see Appendix A.

6 Weiss (1986) has shown that the proposed LM-test is appropriate for non-normal distributions provided some moment conditions are satisfied. The Markow chain test is completely distribution free. 
Table 3.3 ARCH Test Results for Weekly Returns

\begin{tabular}{lccc}
\hline & Commindex & FAZ-index & DS-index DL \\
\hline LM(1) & $53.82^{\circ *}$ & $59.20^{\circ *}$ & $68.52^{\circ *}$ \\
LM(2) & $106.88^{* *}$ & $123.44^{\circ *}$ & $141.98^{\circ *}$ \\
LM(5) & $113.05^{\circ *}$ & $129.69^{* *}$ & $154.62^{* *}$ \\
LRM1 & $24.25^{* *}$ & $22.93^{\circ *}$ & $18.40^{* *}$ \\
LRIM2 & $36.97^{* *}$ & $27.31^{\circ *}$ & $28.91^{\circ *}$ \\
LRM1M2 & $12.72^{* *}$ & 4.38 & $10.51^{* *}$ \\
\hline
\end{tabular}

Table 3.4 ARCH Test Results for Monthlly Returns.

\begin{tabular}{lccc}
\hline & Comm-index & FAZ-index & DS-index DL \\
\hline LM(1) & 0.02 & 0.02 & 0.05 \\
LM(2) & 0.02 & 0.04 & 0.85 \\
LM(5) & 0.43 & 0.29 & 1.03 \\
LRIM11 & 3.48 & 0.60 & 0.91 \\
LRIM2 & 3.53 & 1.02 & 1.68 \\
LRM1M2 & 0.05 & 0.42 & 0.77 \\
\hline
\end{tabular}

Notes for tables 3.3 and $3.4:$ "denotes statistical significance at the $5 \%$ levell, "denotes statistical significance at the $1 \%$ level. The $L M(p)$ test are computed as $T R^{2}$ from a regression of squared residuals an a constant and $p$ lags, and are asympttotically $x^{2}(p)$ distributed. LRIM1 is a Likelihood Ratio test of independence against a first order Markov Chain, and is distributed as $x^{2}(1)$. LRIM2 is a Likelihood Ratio test of independence against a second order Markov Chain , and is distributed as $X^{2}$ (3). LRM11M2 is a Likelihood Ratio test of a first order against a second order Markov Chain, and is distributed as $x^{2}$ (2).

The results of the LM and Markov chain tests for the presence of ARCH in stock returns are given in Tables 3.3 and 3.4. Overall, the evidence presented suggests a fairly consistent rejection of the hypothesis of no heteroskedasticity for weekly stock index return series. In contrast, for monthly stock returns we cannot reject the null hypothesis of no heteroskedasticity in all cases.

\subsection{Modelling German Stock Market Dynamics: Methodology}

In the academic literature it is agreed upon that empirical distributions of stock market returns exhibit fatter tails than one expects from a normal distribution, see for example Akgiray (1989) and Bollerslev et al. (1992). In Tables 3.1 and 3.2 we have provided evidence confirming this fact. All series, on a weekly and monthly basis, suffer from substantial leptokurtosis. These fat tails may be explained by several classes of models. In this chapter we will concentrate on two possible explanations. A first explanation suggests that German stock market index returns can be described by a normal distribution with timevarying parameters (see Hsieh (1989)). An alternative explanation can be offered by the possibility that German stock index returns are generated by a conditionally leptokurtic distribution. These considerations lead us to the maximum likelihood (ML) estimation of the following stochastic processes: 


$$
R_{t}=\varphi_{0}+\varphi_{1} R_{t-1}+\varepsilon_{t}, \quad \varepsilon_{t} \mid \Psi_{t-1} \sim D\left(0, h_{t}\right)
$$

$h_{i}=E_{i-1}\left(\varepsilon_{t}^{2}\right)=\alpha_{0}+\sum_{i=1}^{q} \alpha_{i} \varepsilon_{t-i}^{2}+\sum_{j=1}^{p} \beta_{j} h_{t-j}, \alpha_{0}>0, \alpha_{1}, \beta_{1} \geq 0$

where $\Psi_{t-1}$ is the $\sigma$-field generated by $\left(\varepsilon_{t-1}, \varepsilon_{t-2,}, \ldots\right\}, R$ represents the rate of return defined as the difference in logarithmic value of two consecutive price observations, $\varphi_{0}$ is a constant, $\varphi_{1}$ a parameter to correct for possible first order autocorrelation, $h_{t}$ represents the conditional variance and $D$ can be any symmetric distribution with zero mean and unit variance. The empirical distribution of variables generated by these processes are fat-tailed, compared to the normal distribution. The conditional variance, $h_{t}$, is a linear function of squared lagged residuals and lagged conditional variances. The fact that conditional variances are allowed to depend on past realized variances is particularly consistent with the actual volatility pattern of the stock market where there are both stable and unstable periods.

In the remainder of this chapter we restrict our attention to a $\operatorname{GARCH}(1,1)$ specification since it has been shown to be a parsimonious representation of conditional variance that adequately fits many economic time series, see Bollerslev, Chou and Kroner (1992). Equation 3.3 now reduces to:

$h_{t}=\alpha_{0}+\alpha_{1} \varepsilon_{t-1}^{z}+\beta_{1} h_{t-1}$

The GARCH(1,1) model, given by equations 3.2 and 3.4 , will be estimated under two different assumptions for the conditional error distribution. Most commonly used in GARCH applications is the standard normal distribution. Under conditional normality, the conditional standard deviation can be seen as the stochastic volatility of the process, see Taylor (1994). This has the unfavourable implication that the volatility during a particular time period is known at the end of the previous time period, thereby reducing the impact of news during the period on volatility to zero. An empirical feature of the assumption of conditional normality pertains to the frequent leptokurtosis of standardized GARCHnormal residuals. If conditional normality is appropriate, this phenomenon should not occur. The conditional density function for $\varepsilon_{t}$ under normality reads:

$f_{n}\left(\varepsilon_{t} \mid \Psi_{t-1}\right)=\left(\sqrt{2 \pi h_{t}}\right)^{-1} \exp \left(-\varepsilon_{t}^{2} / 2 h_{t}\right)$

The associated log-likelihood function then becomes:

$L L_{\mu}(\theta)=\frac{1}{2}\left(-T \ln 2 \pi-\sum_{t=1}^{T} \ln h_{t}-\sum_{t=1}^{T} \varepsilon_{t}^{2} / h_{t}\right)$ 
where $\theta$ is the parameter vector. As an alternative to conditional normality we propose a scaled student $t$-distribution. Theoretically and, as will become clear also empirically, this distribution should be preferred in most cases to a conditional normal distribution. The student $t$-distribution is conditionally fat-tailed, a feature which is able to explain - next to conditional heteroskedasticity - unconditional fat tails. Furthermore, it can be seen as a continuous variance mixture of normals, where the mixing variable follows an inverted Gamma-1 distribution. Such a mixture distribution is desirable when information arrives randomly during a time period, see Tauchen and Pitts (1983), because the random character of information is conveyed by the unobservable mixing variable. In this case the stochastic volatility for period $t$ is dependent on unexpected news, and is in general not equal to the conditional standard deviation for period $t$, which is known at the end of period $t-1$. Finally, the observed leptokurtosis of GARCH-residuals does not pose a problem under a conditional $t$-distribution. The conditional density function for $\varepsilon_{i}$ depends on a degrees of freedom parameter $(v)$ and is given by:

$f_{v}\left(\varepsilon_{t} \mid \Psi_{t-1}\right)=\frac{\Gamma\left(\frac{v+1}{2}\right)}{\sqrt{\pi} \Gamma\left(\frac{v}{2}\right)}\left((v-2) h_{t}\right)^{-\frac{1}{2}}\left(1+\frac{\varepsilon_{t}^{2}}{h_{\mathrm{t}}(v-2)}\right)^{\frac{-(v+1)}{2}}, v>2$

The associated log-likelihood function reads:

$$
\begin{aligned}
L L_{v}(\theta)= & T\left(\ln \Gamma\left(\frac{v+1}{2}\right)-\ln \Gamma\left(\frac{v}{2}\right)-\frac{1}{2} \ln (\pi(v-2))\right) \\
& +\sum_{t=1}^{T}\left(-\frac{1}{2} \ln h_{t}-\left(\frac{v+1}{2}\right) \ln \left(1+\frac{\mathcal{E}_{t}^{2}}{(v-2) h_{t}}\right)\right)
\end{aligned}
$$

Here $v$ is added to the parameter vector $\theta$. The scaled $t$-distribution approaches normality when $v \rightarrow \infty$. In addition, we estimate a GARCH(1,1)-in-mean model, initially developed by Engle, Lilien and Robbins (1987), which can be used in addressing questions regarding the risk-return tradeoff in a time series context where the conditional variance may be time-varying. In order to provide empirical evidence for a relationship between stock index returns and conditional variances of the underlying process, the following $\operatorname{GARCH}(1,1)$ in mean model was fitted:

$R_{t}=\varphi_{0}+\varphi_{1} R_{t-1}+\delta h_{t}+\varepsilon_{t}$

$h_{t}=\alpha_{0}+\alpha_{1} \varepsilon_{t-1}^{2}+\beta_{t} h_{t-1}$ 
Again, estimation will be performed under the normal and the student $t$-distribution. Prominent examples of applications of this model to stock index returns are French, Schwert and Stambaugh (1987), Chou (1988) and Baillie and DeGennaro (1990). Although the model has been used extensively, Backus, Gregory and Zin (1989) challenge the usefulness of GARCH in mean models by stating that there is no explicit theoretical relationship between the risk premium and the conditional variance. Furthermore, Pagan and Ullah (1988) point out that the estimates for the parameters in the conditional mean equation are not asymptotically independent from the estimates of the parameters in the conditional variance equation. Misspecification of the GARCH part of the model therefore leads to biases and inconsistencies in the mean part of the model. Consistent with the first criticism, Baillie and DeGennaro (1990) obtained insignificant estimates for $\delta$, in their analysis for daily and monthly return series. In the next section we present and discuss our estimation results for the GARCH and GARCH in mean models respectively.

\subsection{Estimation Procedures and Empirical Results}

Maximum likelihood estimates of the parameters and their heteroskedasticity consistent asymptotic standard errors were obtained by numerical methods using the Berndt, Hall, Hall and Hausman (1974) (BHHH) algorithm ${ }^{7}$. In Tables 3.5 through 3.8 , the estimation results are reported for the stochastic processes described in the previous section. First, we present results for weekly stock indices return series. Inspection of the tables reveals several notable facts. GARCH parameters and autocorrelations coefficients are highly significant for all weekly stock indices and model specifications. For the conditional $t$-distribution, the degrees of freedom parameter $(v)$ is significant in all cases. Note that the sum of the GARCH parameters, $\alpha_{1}+\beta_{1}$, is near unity for all indices, indicating near infinite persistence in the volatility shocks, or IGARCH behaviour, see Engle and Bollerslev (1986). In the case of exchange rates, as conjectured by Lamoureux and Lastrapes (1990b), this may be the result of not accounting for discrete shifts in regimes which affect the level of the unconditional variances, and, therefore can lead to misspecification of the GARCH model ${ }^{8}$. Comparing the results of the standard normal GARCH model to the results of the GARCH-t model, we notice that the volatility persistence has been reduced slightly. For the GARCH-in-mean model, all the estimated models result in statistically

7 All calculations were performed with the software package GAUSS.

\$ Lastrapes (1989) finds that persistence of exchange rate volatility decreases when regime shifts are accounted for, diminishing the likelihood of integrated-in-variance processes. 
insignificant $\delta$ coefficients ${ }^{9}$. Tables 3.7 and 3.8 present the estimation results for monthly stock index return series. It is interesting to note that the monthly estimates of the GARCH models demonstrate less significant parameter coefficients $\left(\alpha_{2}\right)$. This is consistent with the results of the ARCH tests in Tables 3.4, which indicates insignificant ARCH effects in monthly index return series. However, the $\beta_{1}$ estimates remain statistically significant for all indices. The degree of freedom parameter is again significant in all cases and, contrary to what one might expect, does not increase when moving from a weekly to a monthly time interval. This is surprising as the general notion in the financial economics literature suggests that conditional distributions of asset returns, when aggregated, approach normality, see for instance Drost and Nijman (1992) and Nieuwland (1993). If this were indeed true we would expect to see a significant increase in the value of $v$. This is however not the case. For the GARCH in mean model, the estimated $\delta$ coefficients are greater than those for weekly indices returns, but as before the GARCH in mean parameter remains insignificant. Given the results presented above, it is interesting to compare the relative fit of the alternative models. We employ generalized likelihood ratio tests to compare nested models. Such nested models can be tested using the generalized likelihood ratio:

$$
A=\frac{\operatorname{Sup}_{\theta \in \theta_{0}} L(\phi ; x)}{\operatorname{Sup}_{\theta \in \theta} L(\phi ; x)}
$$

of the maximized likelihood values under the null and under the encompassing parameter space, $\Theta$, which also includes the alternative hypothesis. Here, $\mathrm{L}(.$. Is the likelihood function, $\theta$ is the parameter vector and $x$ is the relevant set of observations. Under the null $\Theta_{0}$ the statistic $-2 \ln (\Lambda)$ has a Chi-square distribution with degrees of freedom equal to the difference in the number of parameters between the two models. Thus, the improvement in the likelihood indicates to what extent an enlarged specification helps in fitting the data.

Tables 3.9 and 3.10 present the generalized likelihood ratio tests to compare the relative fit of the models employed. All but nine of the $p$-values associated with the chi-square statistics are close to zero. Thus, the generalized likelihood ratio tests reject the simpler model in favour of the more complicated model in most of the cases. It is clear that for both weekly and monthly return series the student $t$-distribution is superior to the standard normal distribution. For the stock indices, the generalized likelihood ratio tests cannot reject the simpler GARCH model in favour of the more complicated GARCH-in-mean model in the $t$-distribution case.

\footnotetext{
The actual tables are omitted from the text. The results were almost identical to the simple
} GARCH model. 
Table 3.5 GARCH(1,1)-Normal Estimates of Weekly Returns

\begin{tabular}{|c|c|c|c|}
\hline & Comm-index & FAZ-index & DS-index DL \\
\hline$\varphi_{0}\left(.10^{3}\right)$ & $\begin{array}{c}0.871 \\
(0.599)\end{array}$ & $\begin{array}{c}0.982 \\
(0.585)\end{array}$ & $\begin{array}{c}0.786 \\
(0.555)\end{array}$ \\
\hline$\varphi_{i}$ & $\begin{array}{c}0.094^{*} \\
(0.040\}\end{array}$ & $\begin{array}{l}0.112^{\prime} \\
(0.038)\end{array}$ & $\begin{array}{l}0.136^{*} \\
(0.037)\end{array}$ \\
\hline$\alpha_{Q}\left(10^{3}\right)$ & $\begin{array}{c}0.008 \\
(0.006)\end{array}$ & $\begin{array}{c}0.008 \\
(0.007)\end{array}$ & $\begin{array}{c}0.006 \\
(0.005)\end{array}$ \\
\hline$\alpha$ & $\begin{array}{l}0.107^{\circ} \\
(0.045)\end{array}$ & $\begin{array}{l}0.101^{\prime} \\
(0.048)\end{array}$ & $\begin{array}{c}0.089^{\prime} \\
(0.039 y\end{array}$ \\
\hline$\beta_{i}$ & $\begin{array}{l}0.881^{\circ} \\
(0.051)\end{array}$ & $\begin{array}{l}0.884^{\circ} \\
(0.058)\end{array}$ & $\begin{array}{l}0.900^{\circ} \\
(0.047)\end{array}$ \\
\hline $\begin{array}{l}\alpha_{i}+\beta_{i} \\
L L\end{array}$ & $\begin{array}{c}0.988 \\
2506.0\end{array}$ & $\begin{array}{c}0.986 \\
2535.9\end{array}$ & $\begin{array}{c}0.988 \\
2599.6\end{array}$ \\
\hline
\end{tabular}

Table 3.6 GARCH(1,1) t-distribution Estimates of Weekly Returns

\begin{tabular}{lccc}
\hline & Comm-index & FAZ-index & DS-index DL \\
\hline$\varphi_{0}\left(.10^{3}\right)$ & 1.170 & 1.393 & 1.186 \\
& $(0.557)$ & $(0.547)$ & $(0.507)$ \\
$\varphi_{1}$ & 0.074 & 0.088 & 0.120 \\
$\alpha_{0}\left(10^{3}\right)$ & $(0.034)$ & $(0.033)$ & $(0.033)$ \\
& 0.011 & 0.011 & 0.009 \\
$\alpha_{1}$ & $(0.005)$ & $(0.005)$ & $(0.004)$ \\
& 0.104 & 0.098 & 0.089 \\
$\beta_{1}$ & $(0.031)$ & $(0.031)$ & $(0.033)$ \\
& 0.877 & 0.876 & 0.887 \\
$\alpha_{1}+\beta$, & $(0.037)$ & $(0.038)$ & $(0.033)$ \\
$v$ & 0.977 & 0.974 & 0.975 \\
$L$ & 8.735 & 9.086 & 8.281 \\
\hline
\end{tabular}

"and "denote statistical significance at the $1 \%$ and $5 \%$ level respectivelly. Hieteroskedasticity consistent standard errors are given in parentheses. LL denotes the log-Likelihood Value.

Table 3.7 GARCH $(1,1)$-Normal Estimates of Monthly Returns

\begin{tabular}{lccc}
\hline & Comm-index & FAZ-index & DS-index DL \\
\hline$\varphi_{0}\left(.10^{3}\right)$ & 2.231 & 3.178 & 2.977 \\
& $(2.990)$ & $(2.987)$ & $(2.745)$ \\
$\varphi_{\mathrm{r}}$ & 0.063 & 0.077 & 0.087 \\
$\alpha_{i}\left(.10^{3}\right)$ & $(0.062)$ & $(0.063)$ & $(0.061)$ \\
& 0.025 & 0.029 & 0.027 \\
$\alpha_{i}$ & $(0.042)$ & $(0.045)$ & $(0.045)$ \\
$\beta_{i}$ & 0.068 & 0.064 & 0.078 \\
& $(0.038)$ & $(0.041)$ & $(0.048)$ \\
$\alpha_{i}+\beta_{i}$ & $0.929^{*}$ & $0.930^{* *}$ & 0.918 \\
$\mathrm{~L}^{*}$ & $(0.017)$ & $(0.016)$ & $(0.021)$ \\
\hline
\end{tabular}

"and "denote statistical significance at the $1 \%$ and $5 \%$ level respectively. Heteroskedasticity consistent standard errors are given in parentheses. $\mathrm{L}$ denotes the log-Likelihood Value. 
Table 3.8 GARCH(1,1) t-distribution Estimates of Monthly Returns

\begin{tabular}{|c|c|c|c|}
\hline & Comm-index & FAZ-index & DS-index DL \\
\hline$\phi_{0}\left(10^{3}\right)$ & $\begin{array}{c}4.833 \\
(2.954)\end{array}$ & $\begin{array}{c}5.698 \\
(2.866)\end{array}$ & $\begin{array}{c}5.154 \\
(2.758)\end{array}$ \\
\hline$\varphi_{7}$ & $\begin{array}{c}0.043 \\
(0.062)\end{array}$ & $\begin{array}{c}0.063 \\
(0.063)\end{array}$ & $\begin{array}{c}0.071 \\
(0.059)\end{array}$ \\
\hline$a_{0}\left(10^{\overline{3}}\right)$ & $\begin{array}{c}0.050 \\
(0.027)\end{array}$ & $\begin{array}{c}0.052 \\
(0.028)\end{array}$ & $\begin{array}{l}0.047 \\
(0.026)\end{array}$ \\
\hline$a_{i}$ & $\begin{array}{c}0.034 \\
(0.020)\end{array}$ & $\begin{array}{c}0.031 \\
(0.022)\end{array}$ & $\begin{array}{c}0.033 \\
(0.022)\end{array}$ \\
\hline$\beta_{i}$ & $\begin{array}{c}0.943 \\
(0.019)\end{array}$ & $\begin{array}{l}0.941 \\
(0.023)\end{array}$ & $\begin{array}{l}0.940 \\
(0.023)\end{array}$ \\
\hline $\begin{array}{l}\alpha_{1}+\beta_{n} \\
v\end{array}$ & $\begin{array}{l}0.976 \\
6.326\end{array}$ & $\begin{array}{l}0.972 \\
6.446\end{array}$ & $\begin{array}{l}0.973 \\
6.391\end{array}$ \\
\hline
\end{tabular}

" and "denote statistical significance at the $1 \%$ and $5 \%$ level respectively. Heteroskedasticity consistent standard errors are given in parentheses. LL denotes the log-Likelihood Value.

In order to determine the adequacy of the statistical specification, the models are subjected to diagnostic checks on the standardized residuals:

$z_{t}=\frac{\hat{\varepsilon}_{t}}{\sqrt{\hat{h}_{t}}}$,

Here the residuals of the estimation of equation 3.2 are divided by the square root of the estimated conditional variance from equation 3.4. From Jensen's inequality it follows that the standardized residuals, $z_{i}$, should demonstrate less absolute skewness and should be thinner tailed than their unconditional counterparts. Any strong violation of this rule should be regarded as evidence of misspecification, see Hsieh (1989). The diagnostics for the estimated GARCH-t models are presented in Tables 3.11 and 3.12. Overall, the evidence presented suggests a less consistent rejection of the normality hypotheses as compared with the results of Tables 3.1 and 3.2. In addition, we find that in most cases the estimated summary statistics - BJ-test, KS-1 and KS-2 - are smaller than those reported in Tables 3.1 and 3.2 , thus supporting our model specification. In particular, for weekly stock indices the GARCH-t model is reasonably successful at removing excess kurtosis and skewness in all cases. However for the monthly indices, skewness and kurtosis of the standardized residuals are only marginally smaller than their raw data counterparts. In order to test for remaining heteroskedasticity, a residual based test of the models may be carried out by regressing:

$\frac{\left(\hat{\varepsilon}_{t}^{2}-\hat{h}_{t}^{2}\right)}{\hat{h}_{t}^{2}}$ on $\frac{1}{\hat{h}_{t}^{2}}$ 
Table 3.9 Generalized Likelinood Ratio Tests, Weekly Returns

\begin{tabular}{lcccc}
\hline & {$[a] x^{2}(1)$} & {$[b] x^{2}(1)$} & $(c] x^{2}(1)$ & {$[d] x^{2}(1)$} \\
\hline Commindex & 30.76 & 9.86 & 0.00 & 20.74 \\
FAZ-index & $(0.000)$ & $(0.002)$ & $(1.000)$ & $(0.000)$ \\
& 29.40 & 10.06 & 0.04 & 19.38 \\
DS-index & $(0.000)$ & $(0.002)$ & $(0.840)$ & $(0.000)$ \\
& 36.86 & 10.36 & 0.06 & 26.56 \\
& $(0.000)$ & $(0.001)$ & $(0.810)$ & $(0.000)$ \\
\hline
\end{tabular}

Table 3.10 Generalized Likelihood Ratio Tests, Monthly Returns

\begin{tabular}{lcccc}
\hline & {$\left[a \mid x^{2}(1)\right.$} & {$[b] x^{2}(1)$} & {$\left[c \mid x^{2}(1)\right.$} & {$[d] x^{2}(1)$} \\
\hline Comm-index & 26.16 & 0.30 & 0.52 & 26.38 \\
& $(0.000)$ & $(0.580)$ & $(0.470)$ & $(0.000)$ \\
FAZ-index & 30.72 & 0.36 & 0.58 & 30.94 \\
& $(0.000)$ & $(0.550)$ & $(0.450)$ & $(0.000)$ \\
DS-index & 28.56 & 0.34 & 0.44 & 28.76 \\
& $(0.000)$ & $(0.560)$ & $(0.510)$ & $(0.000)$ \\
\hline
\end{tabular}

Notes for tables 3.9 and 3.10: [a] GARCH normal model against GARCH $t$-distribution model; [b] GARCH normal model against GARCH in mean normal model; [C] GARCH t-distribution model against GARCH in mean $t$-distribution madel; [d] GARCH in mean normal model against GARCH in mean $t$-distribution model; $p$-values are given in parentheses.

Table 3.11 Diagnostics for the GARCH $t$-distribution Models, Weekly Returns

\begin{tabular}{|c|c|c|c|}
\hline & Comm-index & FAZ-index & $D S$-index DL \\
\hline Skewness & $-0.38^{*}$ & $-0.47^{*}$ & $-0.49^{* *}$ \\
\hline Kurtosis & $4.60^{* *}$ & $4.64^{\circ *}$ & $4.94^{\circ}$ \\
\hline BJ-test & $133.01^{* \prime}$ & $148.5 .4^{* *}$ & $196.38^{\circ}$ \\
\hline $\mathrm{KS}-1$ & $25.34^{* 4}$ & $36.65^{\circ}$ & $40.11^{\circ}$ \\
\hline $\mathrm{KS}-2$ & $107.67^{\circ}$ & $111.89^{* \mathrm{~m}}$ & $156.27^{*}$ \\
\hline $\mathbb{E}(\mathbb{\|})$ & $8.68^{\circ}$ & 9.26 & $5.82^{\circ}$ \\
\hline $\mathscr{H}(2)$ & $5.91^{* *}$ & $6.41^{* *}$ & $4.52^{\circ *}$ \\
\hline $\mathbb{F}(5)$ & $3.13^{* \prime}$ & $3.53^{* *}$ & $2.69^{\circ}$ \\
\hline
\end{tabular}

Table 3.12 Diagnostics for the GARCH t-distribution Models, Monthly Returns

\begin{tabular}{lccc}
\hline & Comm-index & FAZ-index & DS-index DL \\
\hline Skewness & $-1.20^{* *}$ & $-1.51^{* *}$ & $-1.39^{* *}$ \\
Kurtosis & $10.75^{* *}$ & $12.63^{* *}$ & $12.27^{* *}$ \\
BJ-test & $628.64^{* *}$ & $971.73^{* *}$ & $893.44^{* *}$ \\
KS-1 & $55.48^{* *}$ & $86.47^{* *}$ & $73.73^{* *}$ \\
KS-2 & $573.16^{* *}$ & $885.26^{* *}$ & $819.71^{* *}$ \\
F(1) & 0.06 & 0.02 & 0.03 \\
F(2) & 0.05 & 0.03 & 0.03 \\
$F(5)$ & 0.06 & 0.05 & 0.05 \\
\hline
\end{tabular}

Notes for tables 3.11 and 3.12: The BJ-test denotes the Bera Jarque test for normality; KS-1 and KS-2 pertain to the Kiefer Salmon normality test for skewness and kurtosis respectively. 
and one to two and one to five lags respectively, of the dependent variable and testing whether the estimated coefficients are significantly different from zero by a conventional F-test. The results are reported under $\mathrm{F}(1), \mathrm{F}(2)$ and $\mathrm{F}(5)^{16}$. These statistics follow $F(2,998), F(3,996)$ and $F(6,990)$ distributions for weekly returns and $F(2,226), F(3,224)$ and $F(5,218)$ for monthly series respectively. For the GARCH-t models, rejection of the null hypothesis of no heteroskedasticity occurs for all of the weekly stock index return series, whereas all of the monthly stock indices return series result in statistically insignificant test statistics at the $1 \%$ significance level.

\subsection{Concluding Comments}

In this chapter we have extensively studied the statistical properties of German stock market returns. These properties are important for the pricing of stock options and for international asset pricing models. GARCH models may be used to further understand the relationship between volatility and expected returns.

Our empirical results clearly indicate that for both weekly and monthly series the student $t$-distribution is superior to the standard normal distribution. GARCH-t models fit the data significantly better than the standard normal GARCH using a variety of goodness-of-fit diagnostics. In particular, the estimated GARCH-t models appear to be reasonably successful in accounting for both leptokurtosis and conditional heteroskedasticity from German stock return movements. This does not preclude the possibility that the GARCH- $t$ model for German stock indices is misspecified, but it is the best alternative considered. Furthermore it shows that the estimated GARCH in mean models do not support a statistically significant relationship between a stock index return and its own volatility. Moreover, the empirical finding of near integrated GARCH behaviour suggests that the conditional variances have very long memories and are highly sensitive to initial conditions. Exponential GARCH models may perform better in this respect ${ }^{11}$.

10 See Domowitz and Hakkio (1985) and Pagan and Hall (1983) for a discussion of the general principles leading to such a test statistic.

11 In the Student's Autoregressive model with dynamic heteroskedasticity (STAR) the memory of the conditional variance is as long as the sample while the question of unit roots does not arise. The STAR model has been developed by Spanos (1991, 1992) and successfully applied to exchange rate data by MeGuirk, Robertson and Spanos (1993). The STAR approach seems to be a promising alternative specification to GARCH and may be at fruitful route for further research into the dynamics of German stock market returns. 
Many issues in asset pricing and portfolio allocation decisions can only be meaningfully analysed in a multivariate context. The evidence presented suggests that for multivariate analysis, the assumption of multivariate conditional normality fails to be a reasonable working hypothesis. This issue requires further investigation in future research. The class of GARCH models introduced in this chapter will be evaluated in the next chapter for individual stocks in Germany.

\section{Appendix A Markov Chaïns}

This appendix outlines the construction of the ARCH test based on finite-state Markov chains. A detailed description of the construction of this test can be found in Gregory (1989). Basically this test is equivalent to a test for independence in a two way contingency table. The first step in the construction of the test is to obtain the squared residuals from the estimation of equation 3.1 in the main text. The next step is to apply a discretization rule, by which the squared residuals are divided into different categories or states. The rule applied here is to mark the residuals as being high or low, with the sample median as the boundary. This results in a two-state definition: the squared residuals are either low (state 1) or high (state 2). Next it is assumed that the squared residuals, which now have either state 1 or 2, possesses the Markov property that says that the probability distribution of the squared residuals conditional on its entire past, equals the probability distribution conditional on its first previous value only:

$P\left(e_{t}^{2}=j \mid e_{t-1}^{2}=i, e_{t-2}^{2}, e_{t-3, \ldots}^{2}\right)=P\left(e_{t}^{2}=j \mid e_{t-1}^{2}=i\right)=\lambda_{i j t}$

Where $i, j=1,2$ and $\mathrm{t}=2, \ldots, \mathrm{T}$; $\lambda_{i j l}$ is the probability of being in state $j$ at time $t$, coming from state $i$ at time $t-1$. The transition probabilities are assumed to be time independent, allowing us to define the squared residuals as a homogeneous first order Markov chain. A second order homogeneous Markov chain will also be used to test for second order ARCH effects. In this case the transition probabilities are defined as:

$\lambda_{i j k}=P\left(e_{t}^{2}=k \mid e_{t-1}^{2}=j, e_{t-2}^{2}=i\right) \quad i, j=1,2 \quad t=2, \ldots ., T$

$\lambda_{i j j}$ is the probability of being in state $k$ at time $t$, coming from state $j$ at time $t-1$ and from state $i$ at time $t-2$. The maximum likelihood (ML) estimates of the transition probabilities for the first order Markov chain are determined as $\lambda_{i j}=$ $n_{i j} /\left(n_{i j 1}+n_{i j 2}\right)$, where $n_{i j k}$ is the number of transitions from $i$ to $j$ to $k$. For the first order Markov chain the log-likelihood value at the ML estimates becomes:

$L L M 1=\sum_{i=1}^{2} \sum_{i=1}^{2} n_{i j} \ln \left(n_{i j} / N_{i}\right)$ 
where $N_{i}$ is the number of times that state $i$ is observed. For the second order Markov chain the log-likelihood value at the ML estimates becomes:

$L L M 2=\sum_{i=1}^{2} \sum_{j=1}^{2} \sum_{k=1}^{2} n_{i j k} \ln \left(n_{i j k} / N_{i j}\right)$

where $N_{i j}$ is the number of times that a transition from $i$ to $j$ occurs. These likelihoods are necessary to develop tests for the null hypothesis of homoskedasticity. If this hypothesis were true then there would be no serial dependence in the squared residuals and the transition probabilities would be without any structure. This means that the probability of observing a current state $k$, is independent of previous realizations ( $i$ and $j$ ). This translates into:

$H_{0}: \lambda_{i j}=\lambda_{j}$

when testing independence against a first order Markov chain, and into:

$H_{0}: \lambda_{i j k}=\lambda_{k}$

when testing independence against a second order Markov chain. The ML estimates under both null hypotheses are: $\lambda_{j}=N_{j} / N ; N$ is the total number of observations used in the estimation procedure. The log-likelihood vallue under the null at the ML estimates is:

$L L 1=\sum_{i=1}^{2} N_{i} \ln \left(N_{i} / N\right)$

Two likelihood ratio tests can now be constructed: the first one is a test of independence against a first order homogeneous Markov chain:

$L R(I / M 1)=-2(L L 1-L L M 1) \sim \chi^{2}(1)$

The second one is a test of independence against a second order homogeneous Markov chain:

$L R(I / M 2)=-2(L L 1-L L M 2)-\chi^{2}(3)$

These are the tests that are used in the main text. 


\section{CHAPTER 4}

\section{A Multiplicative GARCH Model for Volatility and Volume}

\subsection{Introduction}

In a recent paper Adrian Pagan (1993) stated: "to get GARCH you need to begin with $G A R C H$ ". This adage in fact indicates that economic models which are able to generate or explain the empirically observed General Autoregressive Conditional Heteroskedastic (GARCH) phenomenon, are very difficult to find. In this chapter we use one of the few theoretical models that potentially could explain GARCH as a starting point in our analysis. This model, based on the Mixture of Distributions Hypothesis (MDH), is subjected to extensive scrutiny and we question some of the implications of this modell. In our opinion, the sources of GARCH have to be identified if we are to make any progress in the time-series modelling of financial data, but we doubt whether the standard mixture model is the appropriate vehicle to do so. More specifically, we think that the motivation to use trading volume as an explanatory variable in GARCH processes, which is based on the MDH, should be further investigated. Nevertheless there appears to be an empirical relationship between daily trading volume and conditional volatility, where trading volume can be interpreted as a time-vary= ing scale factor for volatility.

Diebold (1986) and Diebold and Nerlove (1989) postulated that autocorrelation in the time-varying information flow to the market leads to the well-known time series dependencies in conditional volatility that are fitted by GARCH models. This discussion in fact started with papers of Clark (1973) and Tauchen and Pitts (1983) on subordinated stochastic processes and was followed by Harris' $(1986,1987)$ tests of the mixture of distributions hypothesis. More recently the mixture of distributions theory has been put forward by Lamoureux and Lastrapes (1990a) to explain GARCH in individual stock returns on the New York Stock Exchange (NYSE). This theory is based upon the assumption that daily stock returns are generated from a mixture of distributions, where the rate of information arrival is the stochastic mixing variable. One of the implications of this theory is that stock returns are characterized by a time-varying conditional variance, see Epps and Epps (1976) and Tauchen and Pitts (1983). In 
this framework the mixture hypothesis posits that autocorrelation in the timevarying rate of information arrival leads to the volatility dependencies captured by GARCH-models. Large flows of information tend to be followed by large flows of information and quiet times tend to be followed by quiet times. Since the rate of information arrival is unobservable, several studies used proxies to create empirical testability. One could think of daily trading volume, see Lamoureux and Lastrapes (1990a) (henceforth L\&L) or the dailly number of transactions, see Harris (1987) or even the number of daily price changes, see Laux and $\mathrm{Ng}$ (1993). Lamoureux and Lastrapes (1994) have recently taken a latent variable approach combined with a signal extraction filter to circumvent the before mentioned observability problem. In this chapter we will take a different and fully parametric approach.

In order to provide some validation for the $\mathrm{MDH}$, we present evidence for predictions made by the $\mathrm{MDH}$, using daily stock returns, trading volumes and the number of transactions of 30 stocks listed on the Frankfurt Stock Exchange, where the last two variables represent proxies for the stochastic rate of information arrival. But, although most of the predictions made by the MDH are corroborated by the empirical results, we show that the $\mathrm{MDH}$ builds on assumptions that are quite questionable. The thirty stocks we use, together comprise the well-known German DAX Index. In the first part of our empirical analysis we corroborate the findings of Lamoureux and Lastrapes (1990a) that trading volume is a sufficient statistic for the history of squared returns if it is included additively as a proxy for information arrival in the conditional variance equation of a GARCH specification. However, a serious drawback of conditioning on contemporaneous volume is the possibility of simultaneity bias, which may be the result of a strong contemporaneous correlation between trading volume and price data, see Bollerslev, Chou and Kroner (1992). This issue, together with other problems related to the $\mathrm{MDH}$, causes us to come up with an alternative framework. We will propose a multiplicative relationship between trading volume and the conditional variance of stock returns following a modelling approach presented by Koedijk, Nissen, Schotman and Wolff (1994), and Brenner, Harjes and Kroner (1994). We interpret this model as being loosely inspired by the standard $\mathrm{MDH}$, where we relax or replace some of the original assumptions. As a result we obtain a multiplicative model that assumes a somewhat different role for trading volume than the MDH as in Lamoureux and Lastrapes (1990a).

This chapter is organized as follows: Section 4.2 will give a detailed description of the mixture of distributions hypothesis, its predictions as put forward by Harris (1987), and how it could motivate GARCH estimation. In section 4.3 we

1 'Deutscher Aktienlndex' 
will discuss the limitations of applying the standard mixture of distributions hypothesis and in particular the value of choosing proxies for the rate of information arrival. Our approach starts with the elimination of the unobservable stochastic mixing variable, the rate of information arrival, from the analysis. Having done that we present our multiplicative model based on an adapted version of the $\mathrm{MDH}$. The empirical analysis is contained in sections 4.4 through 4.7. In section 4.4 we provide summary statistics and in section 4.5 we present results for the additive GARCH specification used by Lamoureux and Lastrapes (1990a). In section 4.6 these results are compared with results of the multiplicative specification mentioned above. Various distributions for the conditional error terms will be considered. Moreover, we discuss the interpretation of daily trading volume and the daily number of transactions in GARCH estimation, when both variables are no longer proxies for information arrival. In section 4.7 we eventually estimate the multiplicative model in a weekly context in order to investigate whether the relationship between volatility and volume can also be found in lower frequencies. Section 4.8 presents concluding comments, interpretation of empirical results and suggestions for further research.

\subsection{The Mixture of Distributions Hypothesis and GARCH}

As mentioned before one would like to know what the sources of the observed conditional. heteroskedasticity for financial returns are, or to put it in other words: how can the presence of GARCH be explained. A simple but elegant explanation for daily returns is offered by the mixture of distributions hypothesis. In an environment where a series of events takes place daily, each of which generates information relevant to the pricing of a given stock, every single event, accompanied by new information, leads to price changes and to the adjustment of portfolios through trading activities. The mixture of distributions hypothesis basically relies on the following two assumptions. The first one is that the post-event price changes and corresponding volumes of trade are jointly independently distributed with finite variance, see Harris (1987). The second assumption is that the number of events occurring each day is stochastic. This seems plausible since the arrival and frequency of new information cannot be forecast. For the time being we will follow L\&L (1990a) and assume that daily stock returns are generated from a mixture of distributions and that the rate of daily information arrival is the stochastic mixing variable ${ }^{2}$. Now let $\delta_{\text {it }}$ denote the $i$-th intra-day equilibrium price increment in day $t$. This implies that the daily price increment, $\varepsilon_{i}$, is given by:

2 In section 4.3 we will question some of the assumptions in the strict version of the $\mathrm{MDH}$ and Lamoureux and Lastrapes" (1990a) use of the MDH. 
$\varepsilon_{t}=\sum_{i=1}^{n_{t}} \delta_{i t}$

The random variable $n_{t}$, the number of events on day $t$, is the stochastic mixing variable. It represents the stochastic rate at which new information enters into the market. In this setup it is clear that $\varepsilon_{t}$ is drawn from a mixture of distributions, where the variance of each distribution depends upon information arrival time. L\&Liassume at this point that:

$$
\delta_{i t} \stackrel{\text { i.i.d. }}{\sim} D\left(0, \sigma^{2}\right)
$$

In the stochastic process described above, see Clark (1973), Harris (1987) and $\mathrm{L} \& \mathrm{~L}, \varepsilon_{i}$ is subordinate to $\delta_{i}$ and $n_{t}$ is the directing variable. If the number of information events occurring every day is sufficiently large, then use of the Central Limit Theorem (CLT) results in:

$\varepsilon_{t} \mid n_{t} \sim N\left(0, \sigma^{2} n_{t}\right)$

However, it is by no means certain that the number of information arrivals on any given day is large enough to invoke the results from the CLT. This problem could be avoided by choosing the following assumption:

$$
\delta_{i t} \stackrel{\text { i.i.d. }}{\sim} \quad N\left(0, \sigma^{2}\right)
$$

In this setup 4.3 always holds regardless of the value of $n_{t}$. Note however that 4.3 has very serious implications. The only source of conditional heteroskedasticity for daily stock returns is represented by variation in the number of information arrivals. In this framework the number of information arrivals is the only conditioning variable. This is too restrictive in our view because one should include all relevant information available at time $(t-1)$, not just the rate of information arrival. In section 4.3 we remedy this problem. Let us, however, proceed on the path taken. GARCH could enter the analysis in the following way. The GARCH effects found in stock return series may be a result of time dependence in the evolution rate of equilibrium returns during a trading day. Let us now, analogous to L\&L, assume that the numbers of events on day $t$ are serially correlated ${ }^{3}$. This can be expressed by equation 4.5 :

$$
n_{t}=k+\sum_{j=1}^{p} \beta_{j} n_{t-j}+u_{t}
$$

3 See Chapter 2 for a motivation for this assumption. 
Here $k$ denotes a constant in an AR(p) model, and it is assumed that $u_{t}$ is white noise. Innovations to the stochastic mixing variable, $n_{t}$, persist according to an autoregressive structure. If we now define:

$\Omega_{t}=E\left(\varepsilon_{t}^{2} \mid n_{t}\right)$

then consequently, according to 4.3:

$\Omega_{t}=E\left(\varepsilon_{t}^{2} \mid n_{t}\right)=\sigma^{2} n_{t}$

where the second equality holds if the mixture model is valid. It now follows that:

$\Omega_{t}=\sigma^{2} k+\sum_{j=1}^{q} \beta_{j} \Omega_{t-j}+\sigma^{2} u_{t}$

In essence, equation 4.8 states that the conditional variance of the daily price changes is a function of lagged conditional variances and a white noise term. This type of persistence could possibly be picked up by a GARCH process and it is exactly the specification L\&L use to motivate their additive GARCH-Volume model.

We would like to point out that the link between 4.8 and a full fledged GARCH model is not at all straightforward. The Generalized ARCH model of Bollerslev (1986) allows the conditional variance of a time series to depend upon past squared residuals and past realized conditional variances of the process. It can be interpreted as a reduced form of a more complicated, generally unknown dynamic structure for time-varying conditional second order moments. In its basic form, which has been so successfully employed for time series of high frequency financial returns, a $\operatorname{GARCH}(p, q)$ model can be given by:

$R_{t}=\varphi_{0}+\varphi_{1} R_{t-1}+\varepsilon_{t}, \varepsilon_{t} \mid \Psi_{t-1} \sim D\left(0, h_{t}\right)$

$h_{t}=E_{t-1}\left(\varepsilon_{t}^{2}\right)=\alpha_{0}+\sum_{i=1}^{q} \alpha_{i} \varepsilon_{t-i}^{2}+\sum_{j=1}^{p} \beta_{j} h_{t-j}, \quad \alpha_{0}>0, \quad \alpha_{1}, \beta_{1} \geq 0$

where $\Psi_{t-1}$ is the $\sigma$-field generated by $\left\{\varepsilon_{l-1}, \varepsilon_{t-2}, \ldots\right\}, R_{t}$ represents the rate of return defined as the difference in logarithmic value of two consecutive observations, $\varphi_{0}$ is a constant, $\varphi_{1}$ a parameter to correct for possible autocorrelation, $h_{t}$ represents the conditional variance and $D$ denotes a symmetric distribution. The empirical distribution of variables generated by these processes are fat-tailed, compared to the normal distribution. In the remainder of this chapter, we re- 
strict our attention to a GARCH(1,1) specification ${ }^{4}$, since it has been shown to provide a parsimonious representation of the conditional variance, thoroughly tested in a lot of empirical papers, see the survey by Bollerslev, Chou and Kroner (1992). Equation 4.10 therefore reduces to:

$$
h_{t}=\alpha_{0}+\alpha_{1} \varepsilon_{t-1}^{2}+\beta_{1} h_{t-1}
$$

Before any empirical analysis based on 4.8 can be undertaken, one more problem has to be addressed. The conditional variance specification in 4.8 is taken conditional on $n_{t}$ known. Unfortunately, however, $n_{t}$ is unobservable. One therefore has to choose a proxy for $n_{t}$. Daily trading volume seems to be a logical choice, according to the sequential information model of Copeland (1976) and the mixture model by Epps and Epps (1976). At this point we would like to mention that we believe that trading volume really displays a measure of diversity of interpretation or disagreement of daily information that flows into the market. Whether the MDH is valid or not, we believe that trading volume contains valuable information about the disequilibrium dynamics on stock markets. As is mentioned above, Lamoureux and Lastrapes (1990a) jump from 4.8 to the following specification for the conditional variance:

$$
\begin{aligned}
& R_{t}=\varphi_{0}+\varphi_{1} R_{t-1}+\varepsilon_{t}, \quad \varepsilon_{t} \mid\left(V_{t}, \varepsilon_{t-1}, \varepsilon_{t-2}, \ldots . .\right) \sim N\left(0, h_{t}\right) \\
& h_{t}=\alpha_{0}+\alpha_{1} \varepsilon_{t-1}^{2}+\beta_{1} h_{t-1}+\gamma V_{t}
\end{aligned}
$$

where the conditional variance is parameterized as a function of past squared innovations, the lagged conditional variance, $h_{t-1,}$ and the contemporaneous volume of trade, $V_{t}$ added as a proxy for the stochastic mixing variable ${ }^{5}$. In this model $\gamma$ should be significantly different from zero and positive, in order to comply with the MDH. Furthermore, if the uneven flow of information explains the presence of GARCH effects, the volatility persistence, measured by the sum of $\alpha_{1}$ and $\beta_{1}$, should become significantly smaller than in the simple GARCH $(1,1)$ model". The estimation results in $\mathrm{L} \& \mathrm{~L}$, for a sample of individual NYSE stocks, substantiate these claims. In section 4.5 we will estimate this model specification and a specification where the daily number of transactions, $N_{t}$, is included as an alternative proxy for the speed of information arrival. Equation 4.13 then contains a parameter $\delta$, which measures whether $N_{t}$ can explain the heteroskedasticity in the stock return series.

With $p$ and $i$ equal to one

5 Under the assumption that volume is weakly exogenous in the sense of Engle, Hendry and Richard (1983), see also Lamoureux and Lastrapes (1990a).

6. Another proxy for the mixing variable we will use is the daily number of transactions. This is motivated by the results of Harris' (1987) tests of the mixture of distributions hypothesis. 


\subsection{Limitations and Alternatives of the $\mathrm{MDH}$}

Inspection of the MDH and the L\&L model based on the MDH, as given in section 4.2 , immediately reveals a serious problem. The conditional distribution of the daily equilibrium price increments is unknown. One can use the Central Limit Theorem, to show that this should be a normal distribution, but only if the number of information arrivals on any given day is large enough. There is however no way of knowing this. One way to circumvent this problem is just to assume, see 4.4 , that the intra-daily price increments are normally distributed. This solution is not completely satisfying either. The second problem concerns the distribution of $n_{t}$ itself. Theoretically this should be a discrete distribution, e.g. Poisson, but it is also possible that a continuous distribution could give a close enough approximation. Thirdly, in the mixture hypothesis, the number of information arrivals is the only source of possible conditional heteroskedasticity. In our opinion, as stated earlier, this is far too restrictive. The fourth problem, which is related to the model L\&L propose, is the jump from equation 4.8 to 4.13 , which is only straightforward if:

$$
u_{t}=\varepsilon_{t-1}^{2}
$$

and if daily trading volume or the daily number of transactions are indeed valid proxies for the rate of information arrival. Finally, the L\&L specification is possibly subjected to a simultaneity bias, see for instance Bollerslev, Chou and Kroner (1992). The problems mentioned above motivate us to come up with a different model.

In $L \& L$, the innovations to the mean equation 4.12 were conditionally normal, where trading volume, proxying information arrival, was contained in the information set. One can only assume this, however, if the intra-day equilibrium price increments are normally distributed, or if the Central Limit Theorem can be invoked. Since we are not certain that trading volume or the number of transactions are indeed appropriate proxies for the number of information arrivals, and since we cannot know whether the daily number of information arrivals is large enough to use the CLT, our first approach is to integrate the number of information arrivals out of the problem. This procedure leads to an alternative for conditional normality, the conditional student $t$-distribution. Theoretically and empirically, this distribution of conditional innovations gives better results than the conditional normal distribution, see for instance Baillie and DeGennaro (1990) and Nieuwland (1993) for stock index returns. A conditional student tdistribution is able to explain unconditional fat tails. It can also be seen as a continuous variance mixture of normals, where the mixing variable follows an Inverted Gamma-1 Distribution (henceforth IGD). Tauchen and Pitts (1983) show that this mixture distribution is especially desirable when information 
arrives randomly during a time period, because the random character of information is conveyed by the unobservable stochastic mixing variable, $n_{t}$, in our approach. The stochastic volatility is now dependent on unexpected news. In contrast to the conditional normal distribution, the observed leptokurtosis of GARCH-residuals does not pose a problem under a conditional student $t$-distribution? Therefore, we assume that the rate of information arrival, the stochastic mixing variable, follows an IGD.

The result that a mixture of normal distributions, where the mixing variable follows an IGD, leads to a student $t$-distribution, is well established. The relevance of a student $t$-distribution in a GARCH context was first documented by Bollerslev (1987). He proposed the conditional distribution to permit a distinction between conditional heteroskedasticity and a conditional leptokurtic distribution, either of which could account for the observed unconditional leptokurtosis in high-frequency financial data. Here we explicitly postulate the stochastic rate of information arrival as the mixing variable, whereas Bollerslev (1987) leaves this variable undetermined. The IGD assumption is also motivated by the fact that in the mixture of distributions hypothesis, presented in Section 4.2, the conditional variance is only dependent on $n_{t}$, and we often find IGD's as priors for standard deviations in the Bayesian literature. For our analysis the IGD assumption is essential, but given the motivations above it is a sensible assumption. We start from the assumption that:

$$
\delta_{i t} \mid \Psi_{t-1} \quad-\quad N\left(0, \sigma_{t}^{2}\right)
$$

This allows us to have multiple sources of conditional. heteroskedasticity, as we shall see later on. The intra-day equilibrium price increments have the same conditional distribution within one day, but are able to have different distributions across days ${ }^{8}$. The conditional variance is a function of the elements in the information set, $\Psi_{\mathrm{t}-\mathrm{1}}$. This leads to:

$$
\varepsilon_{t} \mid n_{t}, \Psi_{t-1} \quad \sim \quad N\left(0, \sigma_{t}^{2} n_{t}\right)
$$

The conditional distribution of the daily innovations becomes:

$$
p\left(\varepsilon_{t} \mid \sqrt{n_{t}}, \Psi_{t-1}\right)=\frac{1}{\sqrt{2 \pi} \sigma_{t} \sqrt{n_{t}}} \exp \left(-\frac{\varepsilon_{t}^{2}}{2 \sigma_{t}^{2} n_{t}}\right)
$$

In addition the empirical results in Chapter 3 motivate the choice of the t-distribution.

In essence this means that we assume that the conditional variance of the intra-daily equilibrium price increments is constant during the day, and variable across days. 
To be precise, we assume that the square root of the rate of information arrival follows an inverted gamma distribution:

$p\left(\sqrt{n_{t}}\right)=\frac{2}{\Gamma(\alpha) \gamma^{\alpha} n_{t}^{\alpha+0.5}} \exp \left(-\frac{1}{\gamma n_{t}}\right)$

We also know that:

$p\left(\varepsilon_{t}, \sqrt{n_{t}} \mid \Psi_{t-1}\right)=p\left(\varepsilon_{t} \mid \sqrt{n_{t}}, \Psi_{t-1}\right) \cdot p\left(\sqrt{n_{t}}\right)$

$p\left(\varepsilon_{t} \mid \Psi_{t-1}^{r}\right)=\int_{0}^{\infty} p\left(\varepsilon_{t}, \sqrt{n_{t}} \mid \Psi_{t-1}\right) d \sqrt{n_{t}}$

As it turns out (see Appendix B):

$p\left(\varepsilon_{\mathrm{t}} \mid \Psi_{t-1}\right)=\frac{\Gamma\left(\frac{v+1}{2}\right)}{\Gamma\left(\frac{1}{2}\right) \Gamma\left(\frac{v}{2}\right) \sigma_{t}}\left(1+\frac{\varepsilon_{t}^{2}}{\sigma_{t}^{2}}\right)-\left(\frac{v+1}{2}\right)$

This in fact is a student $t$-distribution with $v$ degrees of freedom, which is in accordance with the observed leptokurtosis in the stock returns. If we let the conditional variance process be a GARCH process, 4.21 becomes:

$p\left(\varepsilon_{t} \mid \Psi_{t-1}\right)=\frac{\Gamma\left(\frac{v+1}{2}\right)}{\Gamma\left(\frac{1}{2}\right) \Gamma\left(\frac{v}{2}\right) \sqrt{h_{t}}}\left(1+\frac{\varepsilon_{t}^{2}}{h_{t}}\right)-\left(\frac{v+1}{2}\right)$

Since we have integrated out the number of information arrivals, we do not necessarily need a proxy for the information evolution rate. The information that is contained in $n_{t}$ is subsumed by the degrees of freedom parameter $v$ (see Appendix B). In this context, trading volume or the number of transactions have no explicit role as a proxy for the rate of information arrival. Any significant inclusion of volume, the number of trades or any other proxy in the conditional variance equation, may suggest that volume and the number of trades provide information over and above the information they provide as a proxy in the conditional normal case. As stated earlier, these proxies could then be viewed as a measure of the diversity of interpretation of the arrived information by the 
market players, in the sense that it displays disagreement about the received information among investors"

We do have to point out that the IGD assumption is just one of many possible distributional assumptions. Since the conditional variance, in the set-up above, is determined by the variables in the information set, and lagged trading volume is in the information set, we can make the conditional variance a function of trading volume, without interpreting trading volume as a proxy for information arrival. Instead we use trading volume as a scale factor for conditional volatility. In particular, we employ a multiplicative model originally developed by Koedijk, Nissen, Schotman and Wolff (1994) (KNSW), and Brenner, Harjes, and Kroner (1994) for modelling the short term interest rate. For our purpose the model is specified as follows ${ }^{10}$ :

$$
\begin{aligned}
& R_{t}=\varphi_{0}+\varphi_{1} R_{t-1}+e_{t^{\prime}} \\
& e_{t} \mid\left(V_{t-1}, e_{t-1^{\prime}} e_{t-2}, \ldots\right) \sim t\left(0, h_{t}, v\right) \\
& \sqrt{h_{t}}=\sigma_{t} V_{t-1}^{\gamma}, \quad e_{t}=\sqrt{h_{t}} \varepsilon_{t}
\end{aligned}
$$

Here trading volume, $V_{t}$, can be interpreted as a time-varying scale factor for volatility, $e_{t}$ is the innovation term ${ }^{11}$ and $\varepsilon_{t}$ student $t$-distributed with zero mean and unit variance:

$\varepsilon_{t} \sim t(0,1, v)$

This specification differs from Lamoureux and Lastrapes (1990a) through the multiplicative relationship between $\sigma_{i}$ and trading volume. Here the variance is generated by the before mentioned GARCH $(1,1)^{12}$ process:

$\sigma_{t}^{2}=\alpha_{0}+\alpha_{1}\left(\sigma_{t-1} \varepsilon_{t-1}\right)^{2}+\beta_{1} \sigma_{t-1}^{2}$

9 A major implication of Epps and Epps (1976) is that trading volume is generated by disagreement on the value of public information. Laux and $\mathrm{Ng}$ (1993) use the daily number of price changes as a proxy for information arrival. In our opinion the number of price quotes could be an appropriate alternative.

10. $V_{1}$ could be replaced here by the number of transactions again, where $\delta$ displays the exponent.

11 Note that $\varepsilon_{1}$ in equation 4.26 is not equal to the $\varepsilon_{1}$ in equation 4.12 .

12 Note that we have to rescale the innovation term in the GARCH(1,1) process for $\sigma_{i}^{2}$. 
Substituting 4.27 in the first part of 4.25 and solving for $\sigma_{t}^{2}$, the conditional variance equation can then be rewritten as (see Appendix $A$ ):

$h_{t}=\alpha_{0} V_{t-1}^{2 \gamma}+\left(\frac{V_{t-1}}{V_{t-2}}\right)^{2 \gamma}\left(\alpha_{1} e_{t-1}^{2}+\beta_{1} h_{t-1}\right)$

A special case of this specification is the GARCH(1,1) model, which holds if $\gamma$ is equal to zero. We expect $\gamma$ to be significantly different from zero and positive, given the established empirical relationship between conditional volatility and trading volume. This multiplicative specification will be estimated and compared with estimates of the additive GARCH specification proposed by $L \& L$. In our specification the possibility of simultaneity bias is eliminated as the conditional variance depends on lagged values of trading volume. In order to further compare our results with the findings of Lamoureux and Lastrapes, we additionally estimate this specification with contemporaneous trading volume. Equation 4.28 then becomes:

$h_{t}=\alpha_{0} V_{t}^{2 \gamma}+\left(\frac{V_{t}}{V_{t-1}}\right)^{2 \gamma}\left(\alpha_{1} e_{t-1}^{2}+\beta_{1} h_{t-1}\right)$

In this setup, contemporaneous trading volume reappears in the conditional variance equation. Although this again implies a possible simultaneity bias, we believe that this bias is less probable given the inclusion of a lagged volume component and the multiplicative specification itself. Finally, in order to capture possible leverage effects in stock returns we estimated an EGARCH model, which was originally proposed by Nelson (1991). We use the following specification, see Taylor (1994):

$R_{t}=\varepsilon_{t} h_{t}, \quad h_{t}=\sigma_{t} V_{t}^{\gamma}, \quad \varepsilon_{t}-N(0,1)$

$\ln \sigma_{t}^{2}=\omega+\varphi \varepsilon_{t-1}+\delta\left(\left|\varepsilon_{t-1}\right|-\sqrt{2 / \pi}\right)+\beta \ln \sigma_{t-1}^{2}$

Asymmetries in the variance equation are reflected in the parameters $\varphi$ and $\delta$, where the total leverage effect is equal to $\varphi / \delta$, see Taylor (1994). In the next section we will present our estimation results, where most specifications will be estimated under a normal and a student $t$-distribution of conditional error terms. 


\subsection{Data and Summary Statistics}

The empirical analysis is performed on a sample of 30 stocks listed on the Frankfurt Stock Exchange in Germany. These stocks comprise the DAX, the well-known German stock market index. We thus have chosen heavily traded, liquid stocks where the variation in daily trading volume is considerable, such that these stocks appear to be a natural testing ground for our model. The period under investigation starts January 10th 1989 and ends July 31st 1992, totalling 886 daily observations. The data were obtained from the Deutsche Finanzdatenbank (DFDB) in Karlsruhe, Germany. For each stock, daily returns, measured as the logarithmic difference between two consecutive observations, corresponding daily volumes of trade and the daily number of transactions were collected. Prices and volumes are adjusted for stock splits, stock dividends, cash dividends and capital modifications.

Table 4.1 Cross-Security Medians of Summary Statistics of Daily Data of a Sample of 30 Stocks listed on the Frankfurt Stock Exchange, January 10, 1989 - July 31, 1992.

\begin{tabular}{|c|c|c|}
\hline Summary Statistic & Daily Data & Adjusted Data \\
\hline Sample Size & 886 & 886 \\
\hline$R_{1}$ skewness & -1.01 & -0.53 \\
\hline$R_{1}$ kurtosis & 18.96 & 13.03 \\
\hline$V$, skewness & 3.05 & \\
\hline$V_{1}$ kurtosis & 19.42 & \\
\hline $\operatorname{Cor}\left(R_{t} V_{v}\right)$ & 0.14 & \\
\hline $\operatorname{Cor}\left(R_{t}^{2}, V_{t}\right)$ & 0.15 & \\
\hline Autocor (1) $R_{t}$ & 0.00 & 0.02 \\
\hline Autocor (1) $R_{t}^{2}$ & 0.15 & 0.18 \\
\hline Autocor (1) V & 0.50 & \\
\hline Autocor (1) $N_{1}$ & 0.68 & \\
\hline $\operatorname{Cor}\left(R_{i,} N_{1}\right)$ & 0.12 & \\
\hline $\operatorname{Cor}\left(\mathbb{R}_{p}^{2}, N_{1}\right)$ & 0.18 & \\
\hline $\operatorname{Cor}\left(V_{n}, N_{n}\right)$ & 0.77 & \\
\hline
\end{tabular}

$R_{i}$ denotes daily returns; $V$ denotes trading volume; $N_{t}$ denotes the number of transactions; $\operatorname{Cor}(\ldots, \ldots)$ denotes the correlation coefficient; Autocor (1).. denotes the first order autocorrelation coefficient.

Inspection of summary statistics of the investigated data set reveals that the hypothesis of an unconditional normal distribution can be rejected for the majority of stocks in this sample. In particular, see Table 4.1 , cross-security median skewness and kurtosis values of daily returns are -1.01 and 18.96 respectively. In addition, daily trading volume also displays substantial skewness and kurtosis. This in fact is consistent with the predictions made by the MDH, see Harris $(1986,1987)$. We also observe significant first order autocorrelations for the squared price changes, daily trading volume and the number of daily trades. Furthermore these variables are highly correlated and virtually all statistics are consistent with the predictions given by the $\mathrm{MDH}$. 
Table 4.2 GARCH Normal Estimates

\begin{tabular}{|c|c|c|c|c|c|c|c|}
\hline & $\varphi_{0}\left(10^{3}\right)$ & $\varphi_{1}$ & $a_{0}\left(.10^{3}\right)$ & $\alpha_{1}$ & $\beta_{i}$ & $\alpha_{1}+\beta_{1}$ & 4 \\
\hline ALL & 0.292 & $0.121^{\circ}$ & 0.004 & $0.163^{\circ}$ & $0.851^{* *}$ & 1.014 & 2492.8 \\
\hline BAS & -0.084 & 0.080 & 0.036 & $0.157^{\circ "}$ & $0.656^{* *}$ & 0.813 & 2590.4 \\
\hline$B A Y$ & 0.205 & 0.069 & 0.014 & $0.246^{\prime \prime}$ & $0.719^{* *}$ & 0.965 & 2611.1 \\
\hline$H Y P^{3}$ & 0.267 & 0.544 & 0.122 & 0.155 & 0.344 & 0.499 & 2456.6 \\
\hline VER & -0.070 & 0.043 & 0.065 & 0.690 & $0.347^{\prime \prime}$ & 0.957 & 2434.4 \\
\hline BMW & 0.239 & $0.141^{\circ}$ & 0.025 & 0.257 & $0.697^{* 4}$ & 0.954 & 2460.1 \\
\hline CBK & -0.254 & 0.035 & 0.014 & 0.077 & $0.874^{\circ *}$ & 0.951 & 2444.9 \\
\hline CON & 0.183 & 0.043 & 0.025 & 0.060 & $0.870^{4 *}$ & 0.930 & 2273.9 \\
\hline DAl & 0.152 & 0.062 & 0.007 & 0.063 & $0.916^{4 "}$ & 0.979 & 2433.6 \\
\hline DEG & 0.508 & 0.091 & 0.194 & 0.366 & 0.035 & 0.401 & 2388.7 \\
\hline$B A B$ & -0.383 & 0.042 & 0.547 & $0.077^{4 \cdot 2}$ & 0.000 & 0.077 & 2039.8 \\
\hline DBK & -0.018 & 0.052 & 0.015 & $0.098^{\circ}$ & $0.838^{* *}$ & 0.936 & 2540.5 \\
\hline DRB & 0.230 & $0.118^{\circ}$ & 0.041 & 0.105 & $0.684^{* *}$ & 0.789 & 2556.9 \\
\hline HEN & -0.073 & 0.018 & 0.126 & $0.084^{\circ}$ & 0.250 & 0.334 & 2547.7 \\
\hline HOE & -0.064 & $0.162^{\circ}$ & 0.042 & 0.395 & $0.482^{\circ}$ & 0.877 & 2572.8 \\
\hline KAR & 0.497 & 0.068 & 0.037 & 0.310 & $0.634^{\circ}$ & 0.944 & 2415.1 \\
\hline KAU & 0.355 & 0.081 & 0.045 & $0.292^{\prime *}$ & $0.607^{\circ *}$ & 0.899 & 2391.4 \\
\hline LIN & -0.171 & $0.173^{\circ *}$ & 0.055 & $0: 236$ & $0.512^{* *}$ & 0.748 & 2584.1 \\
\hline LUF & -0.367 & 0.018 & 0.318 & $0.044^{\circ}$ & 0.233 & 0.277 & 2164.5 \\
\hline MAN & 0.069 & $0.123^{\circ}$ & 0.059 & $0.128^{\circ}$ & $0.735^{24}$ & 0.863 & 2269.3 \\
\hline MMN & 0.111 & $0.126^{\circ}$ & 0.161 & $0.167^{\circ}$ & $0.429^{\prime \prime}$ & 0.596 & 2250.4 \\
\hline MET & 0.036 & 0.089 & 0.183 & 0.081 & 0.401 & 0.482 & 2272.9 \\
\hline PRE & 0.846 & $0.131^{\circ *}$ & 0.048 & 0.084 & $0.761^{\circ}$ & 0.845 & 2337.5 \\
\hline RWE & 0.938 & 0.171 & 0.026 & 0.320 & $0.671^{* a}$ & 0.991 & 2430.3 \\
\hline $\mathrm{SCH}$ & 0.188 & $0.096^{*}$ & 0.010 & $0.078^{\circ}$ & $0.853^{* *}$ & 0.931 & 2699.7 \\
\hline SIE & 0.243 & 0.022 & 0.011 & 0.058 & $0.882^{\prime \prime}$ & 0.940 & 2583.1 \\
\hline $\mathrm{THY}$ & 0.448 & 0.031 & 0.022 & 0.066 & $0.860^{\circ *}$ & 0.926 & 2371.0 \\
\hline VEB & 1.414 & 0.009 & 0.021 & 0.395 & 0.646 & 1.041 & 2461.2 \\
\hline VIA & 0.835 & 0.049 & 0.078 & 0.085 & $0.641^{* 0}$ & 0.726 & 2379.5 \\
\hline WW & 0.140 & 0.048 & 0.053 & $0.053^{\prime \prime}$ & $0.802^{* *}$ & 0.855 & 2264.5 \\
\hline Median & 0.186 & 0.075 & 0.042 & 0.117 & 0.664 & 0.888 & - \\
\hline
\end{tabular}

See equations 4.9 and 4.10 for a detailed description of the parameters presented above. "denotes statistical significance at the $5 \%$ level, "denotes statistical significance at the $1 \%$ level; $L$ denotes the Log. Likelihood value. For a list of explained company abbreviations see Appendix C.

If we adjust the return data by dividing them through the square root of the daily number of transactions, see the procedure mentioned in Harris (1987) and Chapter 2, median values for skewness and kurtosis drop slightly. Normality, however, can still be rejected. In the following sections we will present empirical results for the models outlined in section 4.2. First, we will discuss empirical results of the simple GARCH $(1,1)$ model, without incorporating proxies for the rate of information arrival, under two possible distributions of conditional error terms, the normal distribution and the student $t$-distribution. One also obtains this model as a restricted version of the multiplicative model in equation 4.28 when both $\gamma$ and $\delta$ are restricted to be zero. Then various specifications including trading volume or the number of transactions in the conditional variance equation, both additively and multiplicatively, are estimated, compared, and several hypotheses are tested. 
Table 4.3 GARCH tistibution Estimates

\begin{tabular}{|c|c|c|c|c|c|c|c|c|}
\hline & (e. $\left(10^{3}\right)$ & $\Phi_{1}$ & $\alpha_{1}\left(10^{3}\right)$ & $a_{i}$ & $\beta_{1}$ & $u$ & $\alpha_{1}+\beta_{3}$ & $\mathrm{LL}$ \\
\hline ALL & 0.015 & 0,068 & 0.008 & $0.124^{\circ \prime}$ & $0.858^{\circ}$ & $3.979^{\circ}$ & 0.982 & 25743 \\
\hline BAS & 0.306 & 0.032 & 0.013 & $0.161^{\circ \circ}$ & $0.787^{*}$ & $4066^{* x}$ & 0.948 & 26793 \\
\hline BAY & -0.239 & 0.025 & 0.008 & $0.161^{*}$ & $0.810^{\circ}$ & $4.549^{\circ *}$ & 0.971 & 2679.0 \\
\hline HYP & 0.103 & 0.031 & 0.003 & 0.032 & $0.952^{*}$ & $4.234^{* *}$ & 0.984 & 2587.2 \\
\hline VER & 0.015 & 0.013 & 0.042 & 0.204 & $0.651^{\prime \prime}$ & $4.074^{\circ}$ & 0.855 & 25209 \\
\hline BMW & -0.103 & 0.098 & 0.015 & $0.195^{\text {.8x }}$ & $0.762^{*}$ & $4.448^{\circ *}$ & 0.957 & 2556.5 \\
\hline$C B K$ & .0 .013 & -0.014 & 0.005 & 0.067 & $0.915^{\circ}$ & $4401^{\circ}$ & 0.982 & 2560.3 \\
\hline CON & 0.151 & 0.027 & 0.017 & $0.078^{\prime \prime}$ & $0.880^{\circ \prime}$ & $4.788^{\prime \prime}$ & 0.958 & 2312.2 \\
\hline DAl & -0.134 & 0.048 & 0.001 & $0.038^{\omega *}$ & $0.955^{* *}$ & $5.114^{* 0}$ & 0.993 & 2531.4 \\
\hline DEG & -0.363 & 0.080 & 0.093 & $0.167^{\prime \prime}$ & 0.490 & $3.617^{\circ 6}$ & 0.657 & 2513.6 \\
\hline$B A B$ & -0.770 & 0.017 & 0.278 & $0.284^{*}$ & 0.252 & $3.205^{k-k}$ & 0.536 & 2245.4 \\
\hline DBK & 0.287 & 0.026 & 0.003 & 0.077 & $0.908^{\prime \prime}$ & $5.193^{\circ}$ & 0.985 & 2657.2 \\
\hline DRB & 0.244 & 0.043 & 0.002 & $0.031^{*}$ & $0.960^{\prime \prime}$ & $3.978^{\circ \cdot}$ & 0.991 & 2674.2 \\
\hline HEN & 0.374 & -0.006 & 0.035 & $0.148^{* *}$ & $0.649^{* *}$ & $3.9116^{\circ *}$ & 0.797 & 2705.2 \\
\hline $\mathrm{HOE}$ & .0 .461 & $0.085^{\circ}$ & 0.019 & $0.244^{* \prime}$ & $0.689^{\prime \prime}$ & $4.068^{* *}$ & 0.933 & 2671.0 \\
\hline KAR & -0.007 & $0.904^{\circ}$ & 0.071 & $0.134^{\circ}$ & $0.563^{* *}$ & $4.519^{*}$ & 0.697 & 2518.1 \\
\hline $\mathrm{KAU}$ & 0.016 & 0.063 & 0.086 & $0.272^{i *}$ & $0.431^{\cdots}$ & $5.002^{21 t}$ & 0.703 & 2460.2 \\
\hline LNN & .0 .210 & $0.117^{\circ \circ}$ & 0.036 & $0.321^{*}$ & $0.517^{\ldots 2}$ & $3.716^{* 11}$ & 0.838 & 2747.3 \\
\hline LUF & -0.578 & 0.004 & 0.134 & $0.097^{\circ}$ & $0.614^{\cdots}$ & $3.669^{\circ "}$ & 0.711 & 2257.9 \\
\hline MAN & 0.143 & 0,092 & 0.018 & 0.119 & $0.837^{\prime \prime \prime}$ & $3.698^{\circ \prime \prime}$ & 0.956 & 2447.7 \\
\hline MMN & 0.287 & 0.067 & 0.003 & 0.021 & $0.969^{* 11}$ & $3.831^{\circ *}$ & 0.990 & 2397.6 \\
\hline MET & -0.435 & $0.078^{\circ}$ & 0.076 & $0.189^{\circ}$ & $0.574^{* i n}$ & $4.283^{*}$ & 0.763 & 2417.2 \\
\hline PRE & 0.346 & $0.085^{\circ}$ & 0.013 & 0.096 & $0.872^{\circ *}$ & $4.149^{\circ \prime}$ & 0.968 & 2410.0 \\
\hline RWE & 0.060 & 0.045 & 0.001 & 0.037 & $0.956^{2 *}$ & $3.618^{\prime \prime \prime}$ & 0.993 & 2583.2 \\
\hline $5 \mathrm{CH}$ & 0.182 & $0.075^{\circ}$ & 0.010 & $0.081^{\circ}$ & $0.856^{* *}$ & $3.865^{\circ}$ & 0.937 & 2774.0 \\
\hline SIE & 0.117 & -0.007 & 0.117 & $0.042^{*}$ & $0.946^{\circ *}$ & $5.216^{*}$ & 0.988 & 2688.0 \\
\hline THY & 0.458 & 0.022 & 0.003 & $0.043^{* \prime}$ & $0.946^{* *}$ & $5.171^{\circ}$ & 0.989 & 2432.2 \\
\hline VEB & 0.212 & 0.013 & 0.001 & 0.039 & $0.956^{* *}$ & $3.836^{* *}$ & 0.995 & 2587.1 \\
\hline $\mathrm{V} \mid \mathrm{A}$ & 0.087 & 0.012 & 0.002 & 0.028 & $0.965^{\circ *}$ & $3.462^{*}$ & 0.993 & 2552.6 \\
\hline VW & 0.471 & 0.035 & 0.009 & 0.042 & $0.927^{\circ}$ & $5.051^{\circ}$ & 0.969 & 2363.9 \\
\hline Median & 0.016 & 0.035 & 0.013 & 0.108 & 0.848 & 4112 & 0.963 & \\
\hline
\end{tabular}

See equations 4.9 and 4.10 for a description of the parameters presented abowe. " denotes statistical significance at the $5 \%$ level, " denotes statistical significance at the $1 \%$ level; u represents the degrees of freedom parameter of the associated GARCH $t$-model, $L$ denotes the Log-Likelihood value.

Maximum Likelihood estimates of the various parameters and their heteroskedasticity consistent asymptotic standard errors were obtained by numerical methods using the Berndt, Hall, Hall and Hausmann (1974) (BHHF) algorithm.

\subsection{An Additive Specification}

In Tables 4.2 through 4.7 , estimation results are reported for various additive models. We first estimate a simple GARCH model under a normal distribution of conditional errors. Consistent with our approach in Chapter 3 , we additionally estimate this model under a student $t$-distribution. 
Table 4.4 GARCH Normal + Contemporaneous Volume Estimates

\begin{tabular}{|c|c|c|c|c|c|c|c|c|}
\hline & $\varphi_{0}\left(10^{3}\right)$ & $\varphi_{1}$ & $\alpha_{0}\left(10^{3}\right)$ & $\alpha_{1}$ & 3 & $\times\left(10^{3}\right)$ & $\alpha_{1}+\beta_{1}$ & $\mathrm{LL}$ \\
\hline$\overline{A L L}$ & -0.448 & 0.052 & 0.000 & 0.149 & 0.125 & $4.140^{\circ *}$ & 0.274 & 2523.1 \\
\hline BAS & -0.777 & 0.008 & 0.000 & 0.091 & 0.000 & $0.445^{\circ}$ & 0.091 & 2681.1 \\
\hline BAY & -0.819 & 0.001 & 0.000 & 0.117 & 0.000 & $0.332^{4.4}$ & 0.117 & 26505 \\
\hline HYP & -0.616 & 0.011 & 0.019 & 0.101 & 0.000 & $3.806^{k .4}$ & 0.101 & 2524.6 \\
\hline VER & -0.790 & 0.002 & 0.051 & 0.233 & 0.000 & $2.611^{\circ}$ & 0.233 & 2456.1 \\
\hline BMW & -0.992 & 0.071 & 0.000 & 0.099 & 0,000 & $2.501^{* *}$ & 0.099 & 2520.6 \\
\hline$C B K$ & -1.074 & 0.028 & 0.000 & 0.022 & 0,000 & $0.865^{\ldots *}$ & 0.022 & 2564.8 \\
\hline CON & -1.337 & 0.048 & 0.127 & 0.081 & 0,000 & $1.520^{\mathrm{mx}}$ & 0.081 & 2299.7 \\
\hline $\mathrm{DA}$ & -0.153 & 0.062 & 0.007 & 0.063 & $0.916^{\circ}$ & 0.000 & 0.979 & 24336 \\
\hline DEG & -0.462 & 0.048 & 0.021 & 0.402 & 0.000 & $3.905^{\circ *}$ & 0.402 & 2438.2 \\
\hline$B A B$ & -1.783 & 0.006 & 0.000 & $0.075^{\circ}$ & 0.000 & $7.759^{\prime \prime *}$ & 0.075 & 2227.5 \\
\hline DBK & -0.700 & 0.002 & 0.000 & 0.039 & 0.000 & $0.379^{* *}$ & 0.039 & 2604.8 \\
\hline DRB & -0.759 & 0.036 & 0.000 & 0.035 & 0.000 & $0.895^{* x}$ & 0.035 & 2682.6 \\
\hline HEN & -0.752 & 0.034 & 0.008 & $0.082^{\circ}$ & 0.000 & $4.021^{1 * x}$ & 0.082 & 2658.7 \\
\hline HOE & -1.004 & 0.073 & 0.000 & 0.108 & 0.000 & $0.539^{\prime \prime *}$ & 0.108 & 2651.1 \\
\hline KAR & -0.590 & 0.058 & 0.000 & 0.053 & 0.000 & $4.485^{\circ}$ & 0.053 & 2493.3 \\
\hline KAU & -0.831 & 0.023 & 0.000 & 0.117 & 0.000 & $5.212^{i * *}$ & 0.117 & 2455.9 \\
\hline LIN & -0.577 & 0.077 & 0.000 & 0.134 & 0.000 & $5.246^{1 * *}$ & 0.134 & 2668.1 \\
\hline LUF & -2.067 & 0.015 & 0.035 & 0.032 & 0.000 & $3.939^{\circ *}$ & 0.032 & 2263.8 \\
\hline MAN & -0.862 & $0.107^{*}$ & 0.000 & $0.112^{\circ}$ & 0.000 & $2.743^{4 *}$ & 0.112 & 2412.6 \\
\hline MMN & -0.841 & 0.048 & 0.000 & 0.088 & 0.000 & $0.850^{4 *}$ & 0.088 & 2395.9 \\
\hline MET & -0.853 & 0.039 & 0.027 & $0.080^{\circ}$ & 0.050 & $6.946^{* \prime}$ & 0.130 & 2329.9 \\
\hline PRE & -0.679 & 0.082 & 0.049 & 0.100 & 0.000 & $2.140^{\circ}$ & 0.100 & 2381.0 \\
\hline RWE & -0.293 & 0.061 & 0.000 & 0.099 & 0.000 & $1.374^{\prime \prime}$ & 0.099 & 2491.6 \\
\hline $\mathrm{SCH}$ & -0.300 & $0.110^{\circ}$ & 0.011 & $0.205^{\circ}$ & 0.011 & $0.130^{* *}$ & 0.216 & 2738.7 \\
\hline SIE & -0.592 & 0.054 & 0.000 & 0.129 & 0.000 & $0.289^{\circ *}$ & 0.129 & 2634.0 \\
\hline THY & -1.029 & 0.033 & 0.000 & $0.941^{\prime}$ & 0.000 & $0.719^{\circ *}$ & 0.141 & 2436.5 \\
\hline VEB & -0.480 & 0.035 & 0.000 & 0.094 & 0.000 & $0.618^{*}$ & 0.094 & 2497.5 \\
\hline VIA & -0.586 & 0.009 & 0.000 & $0.045^{* *}$ & 0.000 & $1.714^{\circ}$ & 0.045 & 2484.4 \\
\hline WW & -1.483 & 0.020 & 0.000 & 0.037 & 0.000 & $0.686^{*}$ & 0.037 & 2333.1 \\
\hline Median & -0.768 & 0.038 & 0.000 & 0.097 & 0.000 & 1.617 & 0.100 & - \\
\hline
\end{tabular}

See equations 4.12 and 4.13 for a description of the parameters abowe; y represents the volume parameter, $U$ represents the degrees of freedom parameter and $L$ denotes the Log-Likelihood value.

In Table 4.2 results for the normal specification are reported. "The GARCHparameters, $\alpha_{1}$ and $\beta_{1}$, are statistically significant in 12 and 23 cases respectively ${ }^{13}$. Table 4.3 presents results for the student $t$-distribution. Here, $\alpha_{1}$ is significant in 19, and $\beta_{1}$ in 28 out of 30 cases. The degree of freedom parameter, $v_{r}$ is significant at the $1 \%$ level in every single case. Median estimates for the persistence in volatility $\left(\alpha_{1}+\beta_{1}\right)$ are 0.888 for the normal model and 0.963 for the student $t$-model. This results favours the hypothesis that there is time-varying volatility in daily German stock returns. Furthermore it confirms our believe that a $t$-distribution generally provides a better fit.

13 It should be noted that, in the normal case, an IGARCH specification should be estimated for ALL and VEB, as the sum of the GARCH-parameters exceeds unity. 
Table 4.5 GARCH t-distribution + Contemporaneous Volume Estimates

\begin{tabular}{|c|c|c|c|c|c|c|c|c|c|}
\hline & $\varphi_{0}\left(10^{3}\right)$ & $\Phi$ & $a_{0}\left(.10^{3}\right)$ & $\alpha_{1}$ & $\beta_{3}$ & $y\left(.10^{3}\right)$ & $u$ & $\alpha_{1}+\beta_{1}$ & LL \\
\hline ALL. & -0.406 & 0.050 & 0.017 & $0.217^{\circ}$ & 0.204 & 2.905 & $4.394^{* *}$ & 0.421 & 2575.1 \\
\hline BAS & -0.777 & 0.015 & 0.000 & 0.103 & 0,000 & 0.437 & $6.225^{*}$ & 0.103 & 2708.2 \\
\hline BAY & -0.246 & 0.025 & 0.005 & $0.185^{\circ .3}$ & 0.774 & 0.012 & $4.610^{* *}$ & 0.956 & 2680.5 \\
\hline HYP & -0.414 & 0.019 & 0.056 & $0.099^{\circ}$ & 0.000 & $2.468^{\circ}$ & $4.910^{* *}$ & 0.099 & 2597.8 \\
\hline WER & -0.281 & 0.003 & 0.052 & $0.282^{* *}$ & 0.344 & $0.789^{\circ . *}$ & $4.286^{\circ "}$ & 0.626 & 2525.1 \\
\hline BMW & -0.926 & 0.056 & 0.000 & 0.126 & 0.000 & $2.354^{4 * *}$ & $5.000^{* *}$ & 0.126 & 2576.4 \\
\hline CBK & -0.922 & -0.031 & 0.006 & 0.049 & 0.000 & $0.794^{* *}$ & $7.118^{* *}$ & 0.049 & 2588.1 \\
\hline CON & -1.167 & 0.014 & 0.087 & $0.146^{\circ *}$ & 0.000 & $1.789^{\prime \prime *}$ & $5.169^{* *}$ & 0.146 & 2337.3 \\
\hline DAI & -0.240 & 0.456 & 0.000 & $0.038^{\circ}$ & $0.960^{\circ \circ}$ & 0.000 & $5.079^{\circ}$ & 0.998 & 2531.5 \\
\hline DEG & 0.925 & 0.040 & 0.021 & $0.120^{\circ *}$ & 0.000 & $4.037^{* 2}$ & $4.950^{\circ}$ & 0.120 & 2547.5 \\
\hline$B A B$ & -1.743 & -0.009 & 0.000 & $0.140^{\circ}$ & 0.000 & $6.595^{\circ *}$ & $4.922^{\circ}$ & 0.140 & 2313.9 \\
\hline DBK & -0.369 & 0.010 & 0.016 & 0.084 & 0.000 & $0.303^{* *}$ & $6.061^{* 2}$ & 0.084 & 2657.2 \\
\hline DRB & -0.481 & 0.016 & 0.000 & 0.076 & 0.000 & $0.830^{\circ *}$ & $6.498^{*}$ & 0.076 & 2719.0 \\
\hline HEN & -0.262 & -0.022 & 0.030 & 0.107 & 0.000 & $2.947^{* *}$ & $4.627^{*}$ & 0.107 & 2724.4 \\
\hline HOE & -0.768 & 0.059 & 0.000 & $0.223^{\circ}$ & 0.175 & 0.366 & $5.260^{\prime 2}$ & 0.398 & 2693.7 \\
\hline KAR & -0.522 & 0.065 & 0.023 & 0.054 & 0.000 & $3.627^{* 0}$ & $5.984^{\circ}$ & 0.054 & 2544.4 \\
\hline KAU & -0.628 & 0.034 & 0.010 & $0.170^{\circ}$ & 0.037 & $4.248^{* *}$ & $6.285^{* *}$ & 0.207 & 2492.3 \\
\hline LIN & -0.531 & 0.097 & 0.006 & 0.294 & 0.046 & $3.580^{\circ}$ & $4.275^{\circ}$ & 0.340 & 2766.2 \\
\hline LUF & .1 .650 & -0.009 & 0.041 & $0.047^{*}$ & 0.000 & $3.643^{* *}$ & $5.051^{*}$ & 0.047 & 2314.8 \\
\hline MAN & -0.470 & 0.093 & 0.000 & $0.190^{* *}$ & 0.000 & $2.430^{\circ *}$ & $4.540^{\circ *}$ & 0.190 & 2487.4 \\
\hline $\mathbb{M} M \mathbb{N}$ & -0.716 & 0.044 & 0.000 & $0.090^{\circ}$ & 0.000 & $0.807^{* *}$ & $5.970^{\circ *}$ & 0.090 & 2443.3 \\
\hline MET & -0.797 & 0.064 & 0.051 & $0.149^{\circ *}$ & 0.000 & $4.810^{* *}$ & $5.107^{* *}$ & 0.1149 & 2445.6 \\
\hline PRE & -0.686 & 0.059 & 0.046 & $0.128^{*}$ & 0.000 & $2.237^{*}$ & $4.252^{\circ}$ & 0.128 & 2427.0 \\
\hline RWE & -0.562 & 0.052 & 0.000 & $0.150^{\circ}$ & 0.000 & $1.237^{* *}$ & $4.091^{* \prime}$ & 0.150 & 2586.1 \\
\hline $\mathrm{SCH}$ & -0.162 & 0.072 & 0.010 & $0.207^{* *}$ & 0.106 & $1.150^{\circ}$ & $4.347^{* *}$ & 0.313 & 2788.1 \\
\hline S俛 & -0.433 & 0.033 & 0.012 & $0.181^{\circ}$ & 0.000 & 0.237 & $5.707^{* *}$ & 0.150 & 2688.0 \\
\hline THY & .0 .933 & 0.017 & 0.000 & $0.131^{\circ}$ & 0.000 & $0.718^{* *}$ & $7.133^{* x}$ & 0.131 & 2458.4 \\
\hline VEB & -0.465 & 0.028 & 0.005 & 0.131 & 0.000 & $0.551^{\circ *}$ & $4.218^{* *}$ & 0.131 & 2587.1 \\
\hline VIA & -0.680 & -0.005 & 0.000 & $0.102^{\circ *}$ & 0.000 & $1.502^{\circ}$ & $4.223^{*}$ & 0.102 & 2582.2 \\
\hline WN & -1.057 & 0.031 & 0.023 & $0.066^{\circ}$ & 0.000 & $0.556^{*}$ & $6.890^{\circ}$ & 0.066 & 2383.2 \\
\hline Median & -0.628 & 0.031 & 0.006 & 0.130 & 0.000 & 1.646 & 5.026 & 0.130 & - \\
\hline
\end{tabular}

See equations 4.12 and 4.13 for a description of the parameters above; yrepresents the wolume parameter, urepresents the degrees of freedom parameter and $L L$ denotes the Log-Likelihood value.

Table 4.4 shows the results for a GARCH $(1,1)$ normal model including daily trading volume additively in the conditional variance equation as a proxy for the unobservable rate of information arrival (the $\mathrm{L} \& \mathrm{~L}$ model) ${ }^{14}$. It is rather striking to see that median values of $\beta_{1}$ drop to zero and median values of the sum of the GARCH-parameters also severely decrease from 0.888 (Table 4.2) to 0.100 (Table 4.4). Especially the significance of $\beta_{1}$ decreases: it remains significant in only one case. The volume parameter, $\gamma$, is significant in 28 out of 30 cases. Results for the GARCH $t$-distribution model, see Table 4.5, confirm this results. Here, the sum of the GARCH parameters decreases from 0.963 (Table 4.3 ) to 0.130 (Table 4.5 ).

14 Actual values of the daily number of transactions and daily trading volume in equation 4.13 are divided by $10^{3}$ and $10^{6}$ respectively. 
Table 4.6 GARCH Normal + Number of Transactions Estimates

\begin{tabular}{|c|c|c|c|c|c|c|c|c|}
\hline & $\varphi_{0}\left(10^{3}\right)$ & $\varphi_{1}$ & $\alpha_{0}\left(10^{3}\right)$ & $\alpha_{1}$ & $\beta_{1}$ & $0\left(10^{3}\right)$ & $\alpha_{1}+\beta_{1}$ & $\mathrm{LL}$ \\
\hline AlLL & -0.332 & 0.071 & 0.010 & 0.139 & 0.149 & $0.408^{\circ}$ & 0.288 & 2502.7 \\
\hline BAS & -0.499 & 0.038 & 0.013 & 0.093 & 0.000 & $0.166^{\circ}$ & 0.093 & 2649.8 \\
\hline BAY & +0.479 & 0.028 & 0.013 & 0.206 & 0.021 & $0.140^{\circ}$ & 0.227 & 2628.8 \\
\hline HYP & -0.172 & 0.036 & 0.035 & 0.220 & 0.000 & $0.679^{\circ}$ & 0.220 & 2502.0 \\
\hline VER & -0.007 & 0.033 & 0.007 & 0.452 & $0.244^{*}$ & $0.342^{\circ}$ & 0.696 & 2524.8 \\
\hline BMW & -0.605 & 0.079 & 0.025 & 0.105 & 0.000 & $0.550^{\circ *}$ & 0.105 & 2563.0 \\
\hline CBK & -0.341 & 0.010 & 0.014 & 0.027 & 0.000 & $0.313^{* *}$ & 0.027 & 2555.7 \\
\hline $\operatorname{CON}$ & -1.146 & 0.042 & 0.035 & $0.075^{\prime \prime}$ & 0.000 & $0.725^{\circ *}$ & 0.075 & 2322.6 \\
\hline DAl & -0.838 & $0.107^{*}$ & 0.031 & $0.182^{\prime \prime}$ & 0.000 & $0.156^{* *}$ & 0.182 & 2450.4 \\
\hline DEG & 0.181 & 0.063 & 0.049 & 0.448 & 0.000 & $0.554^{* *}$ & 0.448 & 2414.1 \\
\hline$B A B$ & -0.791 & 0.004 & 0.008 & 0.085 & 0.000 & $1.764^{* *}$ & 0.085 & 2178.4 \\
\hline DBK & -0.306 & 0.038 & 0.024 & 0.090 & 0.000 & $0.104^{*}$ & 0.090 & 2591.9 \\
\hline DRB & -0.392 & 0.066 & 0.013 & 0.049 & 0.000 & 0.344 & 0.049 & 2651.0 \\
\hline HEN & -0.484 & 0.023 & 0.016 & $0.080^{\prime \prime}$ & 0.000 & $0.690^{\circ *}$ & 0.080 & 26292 \\
\hline HOE & -0.556 & $0.102^{*}$ & 0.014 & 0.152 & 0.000 & $0.207^{* *}$ & 0.152 & 2619.0 \\
\hline KAR & -0.018 & $0.115^{\circ}$ & 0.030 & 0.125 & 0.036 & $0.672^{* *}$ & $0.16 \|$ & 2450.4 \\
\hline $\mathrm{KAU}$ & -0.316 & 0.068 & 0.032 & 0.118 & 0.000 & $0.823^{* *}$ & 0.118 & 2429.5 \\
\hline LIN & -0.342 & 0.894 & 0.023 & 0.130 & 0.000 & $0.757^{* *}$ & 0.130 & 2617.6 \\
\hline LUF & -1.126 & 0.009 & 0.032 & 0.043 & 0.000 & $1.158^{* *}$ & 0.043 & 2259.5 \\
\hline MAN & -0.070 & $0.111^{\circ}$ & 0.025 & $0.085^{\circ}$ & $0: 000$ & $0.655^{* *}$ & 0.085 & 2361.8 \\
\hline MMN & -0.101 & 0.059 & 0.020 & 0.077 & 0.000 & $0.352^{* *}$ & 0.077 & 2367.0 \\
\hline MET & -0.360 & 0.071 & 0.113 & 0.063 & 0.004 & $0.936^{*}$ & 0.067 & 2295.4 \\
\hline PRE & 0.129 & $0.112^{\star}$ & 0.040 & 0.218 & 0.041 & $0.508^{*}$ & 0.259 & 2372.6 \\
\hline RWE & 0.274 & 0.098 & 0.025 & 0.208 & 0,000 & $0.393^{*}$ & 0.208 & 2464.2 \\
\hline $\mathrm{SCH}$ & -0.197 & 0.105 & 0.000 & 0.167 & 0.000 & 0.296 & 0.167 & 2731.7 \\
\hline SIE & -0.111 & 0.070 & 0.022 & 0.179 & 0.000 & $0.087^{\circ}$ & 0.179 & 2626.0 \\
\hline THY & -0.181 & 0.036 & 0.043 & $0.103^{*}$ & 0.000 & $0.272^{\circ}$ & 0.103 & 2414.5 \\
\hline VEB & 0.156 & 0.050 & 0.021 & 0.110 & 0.000 & $0.226^{\circ}$ & 0.110 & 2478.7 \\
\hline VIA & 0.056 & 0.018 & 0.048 & 0.051 & 0.000 & $0.474^{\circ}$ & 0.051 & 2428.7 \\
\hline WN & -0.905 & 0.031 & 0.045 & 0.036 & 0.000 & $0.228^{\circ}$ & 0.036 & 2331.7 \\
\hline Me & -0.324 & 0.061 & 0.025 & 0.108 & 0.000 & 0.401 & 0.108 & - \\
\hline
\end{tabular}

See equations 4.12 and 4.13 for a detaled description of the parameters presented above; $\delta$ represents the volume parameter, $L L$ denotes the Log-Likelihood value.

The volume parameter is significant in 24 cases and the degrees of freedom parameter remains significant in every case. It is quite interesting to see that $\alpha_{1}$ remains significant where the volume parameter $\gamma$ is not significant ${ }^{15}$. Estimates of a restricted model, where the GARCH-parameters $\alpha_{1}$ and $\beta_{1}$ are restricted to be zero, are also estimated, but for reasons of space these tables are omitted.

15 In addition we estimated GARCH models for the German index (DAX). A simple GARCH$(1,1)$ model with a conditional t-distribution showed significant parameter values. Including daily aggregated market volume or aggregated market transactions in the conditional variance equation did not improve empiricall results. Apparently, the relationship between volume and volatility is stronger at the individual stock level than at an aggregated level. Furthermore the estimation of an additive model, where volume's exponent is estimated, yields an estimated exponent that is not significantly different from one in the majority of cases. 
Table 4.7 GARCH t-distribution + Number of Transactions Estimates

\begin{tabular}{|c|c|c|c|c|c|c|c|c|c|}
\hline & $\varphi_{0}\left(.10^{3}\right)$ & $\varphi_{1}$ & $C_{o}\left(.10^{3}\right)$ & $\alpha_{1}$ & $\beta_{i}$ & $8\left(10^{3}\right)$ & $u$ & $a_{1}+\beta_{i}$ & $\mathrm{LL}$ \\
\hline $\mathbb{A L L}$ & 0.015 & 0.068 & 0.008 & 0.124 & $0.858^{\circ}$ & 0.000 & $3.979^{\circ}$ & 0.982 & 2574.3 \\
\hline BAS & -0.570 & 0.038 & 0.001 & $0.231 "$ & 0.244 & 0.112 & $4.866^{\circ}$ & 0.475 & 2691.4 \\
\hline BAY & -0.260 & 0.025 & 0.004 & $0.181^{* *}$ & $0.779^{\circ}$ & 0.008 & $4.587^{* 4}$ & 0.960 & 2680.0 \\
\hline$H_{Y P}$ & .0 .274 & 0.032 & 0.063 & $0.109^{*}$ & 0.000 & $0.524^{\circ *}$ & $4.507^{* *}$ & 0.109 & 2592.0 \\
\hline VER & -0.231 & 0.006 & 0.044 & $0.273^{* *}$ & 0.341 & 0.240 & $4.250^{\circ *}$ & 0.614 & 2524.8 \\
\hline$B M W$ & -0.333 & 0.094 & 0.000 & $0.294^{n *}$ & $0.483^{\circ}$ & 0.190 & $4.497^{*}$ & 0.777 & 2563.0 \\
\hline CBK & -0.335 & 0.015 & 0.018 & 0.057 & 0.000 & $0.286^{* *}$ & $6.851^{\circ *}$ & 0.057 & 2584.7 \\
\hline CON & .1 .066 & 0.017 & 0.022 & $0.125^{* *}$ & 0.000 & $0.730^{n a+}$ & $6.106^{\circ *}$ & 0.125 & 2347.5 \\
\hline DAI & -0.144 & 0.048 & 0.001 & $0.036^{* *}$ & $0.959^{* *}$ & 0.000 & $5.110^{* *}$ & 0.995 & 2531.6 \\
\hline DEG & .0 .603 & 0,062 & 0.035 & $0.116^{*}$ & 0.000 & $0.675^{\circ}$ & $4.283^{\prime \prime}$ & 0.116 & 2535.6 \\
\hline $\mathrm{BAB}$ & -9.016 & 0.008 & 0.006 & $0.132^{*}$ & 0.000 & $1.411^{\circ}$ & $4.639^{\circ}$ & 0.132 & 2302.9 \\
\hline DBK & -0.078 & 0.033 & 0.030 & $0.130^{\circ}$ & 0.000 & $0.089^{* *}$ & $5.686^{* *}$ & 0.130 & 2644.3 \\
\hline DRB & $=0.111$ & 0.037 & 0.012 & 0.107 & 0.000 & 0.310 & $5.355^{\circ}$ & 0.107 & 2705.0 \\
\hline$H E N$ & -0.065 & 0.018 & 0.032 & $0.102^{*}$ & 0.000 & $0.506^{\circ 4}$ & $4.639^{\circ}$ & 0.102 & 2719.2 \\
\hline HOE & -0.603 & $0.081^{*}$ & 0.000 & $0.285^{* 0}$ & $0.452^{\circ *}$ & $0.081^{\circ}$ & $4.531^{\circ}$ & 0.737 & 2684.4 \\
\hline KAR & -0.277 & $0.086^{\circ}$ & 0.039 & 0.089 & 0.000 & $0.603^{\circ}$ & $5.108^{\circ *}$ & 0.089 & 2535.5 \\
\hline KAU & $=0.290$ & 0.055 & 0.025 & $0.213^{* *}$ & 0.081 & $0.645^{\circ *}$ & $5.602^{\circ *}$ & 0.294 & 2482.7 \\
\hline $\mathbb{L N}$ & -0.286 & $0.116^{* *}$ & 0.016 & 51 & 0.318 & 0.261 & $40^{\circ *}$ & 0.669 & 2753.3 \\
\hline LUF & -1.167 & 0.011 & 0.023 & 0.046 & 0.000 & 1.167 & $5.016^{\circ}$ & 0.046 & 2310.5 \\
\hline MAN & -0.104 & $0.100^{* *}$ & 0.026 & $0.180^{* 8}$ & 0.000 & $0.533^{* *}$ & $4.054^{*}$ & 0.180 & 2474.1 \\
\hline MMMN & -0.242 & 0.053 & 0.017 & 0.096 & 0.000 & $0.331^{* *+}$ & $5.021^{* *}$ & 0.0916 & 2430.4 \\
\hline MET & -0.485 & $0.079^{\circ}$ & 0.071 & $0.151^{*}$ & 0.000 & $0.784^{* *}$ & $4.530^{\circ}$ & 0.151 & 2438.1 \\
\hline PRE & -0.380 & 0.079 & 0.033 & 0.161 & 0.000 & $0.635^{* \ldots}$ & $4.110^{\circ *}$ & 0.161 & 2423.1 \\
\hline RWE & -0.315 & 0.060 & 0.019 & 0.176 & 0.000 & $0.398^{* *}$ & $3.657^{* *}$ & 0.176 & 2576.7 \\
\hline $5 \mathrm{CH}$ & -0.134 & 0.067 & 0.000 & $0.166^{\circ}$ & 0.000 & $0.305^{* *}$ & $4.081^{\circ *}$ & 0.166 & 2790.7 \\
\hline $5 \mathrm{SE}$ & -0.115 & 0.010 & 0.001 & $0.034^{* *}$ & $0.958^{\circ}$ & 0.000 & $5.222^{\circ *}$ & 0.992 & 2662.0 \\
\hline THY & $\cdot 0.386$ & 0.028 & 0.018 & $0.117^{* *}$ & 0.000 & $0.300^{\circ}$ & $6.362^{\circ *}$ & 0.117 & .2448 .6 \\
\hline VEB & -0.192 & 0.032 & 0.025 & $0.145^{*}$ & 0.000 & $0.202^{* \prime \prime}$ & $3.928^{\circ *}$ & 0.145 & 2574.3 \\
\hline VIA & -0.435 & 0.013 & 0.029 & $0.141^{\circ \circ}$ & 0.000 & $0.455^{\circ}$ & $3.509^{* *}$ & 0.141 & 2556.7 \\
\hline WW & -0.560 & 0.039 & 0.041 & 0.061 & 0.000 & $0.206^{* \prime \prime}$ & $6.658^{* *}$ & 0.061 & 2385.6 \\
\hline Median & -0.288 & 0.039 & 0.021 & 0.131 & 0.000 & 0.308 & 4.613 & 0.148 & - \\
\hline
\end{tabular}

See equations 4.12 and 4.13 for a description of the parameters above; 8 represents the wolume parameter, $u$ represents the degrees of freedom parameter and $L L$ denotes the Log-Likelihood value.

However, it is interesting to note that the volume parameter is statistically significant at the $1 \%$ level in every single case in the restricted specification. A brief comparison of the estimates of both specifications (restricted and unrestricted) shows that $\gamma$ tends to rise in value as well. The results above confirm the findings of Lamoureux and Lastrapes (1990a), who estimate an additive specification with a normal distribution of conditional error terms. Consistent with their results, we find a sharp decrease in the persistence of volatility when including trading volume in the conditional variance equation. Likelihood ratio tests show that the student $t$-distribution model outperforms the normal model in almost every single case. Tables 4.6 and 4.7 provide estimation results for the additive specification including the daily number of transactions as a proxy. Results are more or less the same: GARCH-parameters drop in value and significance, the transactions parameter, $\delta_{v}$ is significant in the majority of cases. 
Table 4.8 GARCH t-distribution + Lagged Volume Estimates (Multiplicative)

\begin{tabular}{|c|c|c|c|c|c|c|c|c|c|}
\hline & $\varphi_{0}\left(10^{3}\right)$ & $\varphi_{i}$ & $a_{0}\left(10^{3}\right)$ & $a_{1}$ & $\beta_{4}$ & $y$ & 0 & $\alpha_{1}+\beta_{3}$ & LL \\
\hline $\mathrm{ALL}$ & 0.064 & 0.065 & 0.290 & 0.084 & $0.9112^{\circ}$ & $0.225^{\circ}$ & $4.280^{\circ}$ & 0.996 & 2581.1 \\
\hline BAS & -0.243 & 0.045 & 0.743 & 0.100 & $0.867^{* *}$ & $0.179^{\circ}$ & $4.363^{\circ *}$ & 0.967 & 2683.2 \\
\hline BAY & -0.161 & 0.023 & 0.555 & $0.119^{\circ}$ & $0.859^{* * *}$ & $0.155^{\circ}$ & $4.750^{\circ *}$ & 0.978 & 2681.6 \\
\hline HYP & 0.081 & 0.033 & 0.108 & 0.023 & $0.970^{\circ *}$ & $0.106^{*}$ & $4.443^{\prime \prime \prime}$ & 0.993 & 2590.2 \\
\hline VER & 0.108 & 0.009 & 0.053 & 0.018 & $0.978^{1 * *}$ & $0.095^{\circ}$ & $4.240^{\circ *}$ & 0.996 & 2527.1 \\
\hline BMW & 0.092 & $0.101^{\mathrm{ma}}$ & 0.952 & $0.116^{\circ}$ & $0.853^{* *}$ & $0.205^{\circ}$ & $4.744^{24}$ & 0.968 & 2562.7 \\
\hline CBK & 0.093 & -0.018 & 0.188 & 0.039 & $0.953^{* *}$ & $0.141^{* *}$ & $4.528^{\circ \prime \prime}$ & 0.992 & 2564.0 \\
\hline CON & 0.201 & 0.026 & 0.927 & $0.055^{\circ}$ & $0.925^{* *}$ & $0.130^{\circ}$ & $4.978^{\circ *}$ & 0.980 & 2317.8 \\
\hline DAl & -0.163 & 0.048 & 0.040 & $0.028^{8 *}$ & $0.968^{\circ *}$ & $0.144^{\circ}$ & $5.179^{10}$ & 0.996 & 2535.1 \\
\hline DEG & -0.279 & 0.075 & 1.131 & 0.051 & $0.907^{7.6}$ & 0.066 & $3.636^{*}$ & 0.958 & 2515.7 \\
\hline BAB & -0.724 & 0.017 & $3.692^{\circ "}$ & $0.153^{*}$ & 0.202 & $0.237^{*}$ & $3.307^{* 4}$ & 0.355 & 2255.7 \\
\hline DBK & 0.286 & 0.027 & 0.167 & 0.058 & $0.933^{m \circ}$ & 0.089 & $5.292^{* 4}$ & 0.991 & 2658.3 \\
\hline DRB & 0.252 & 0.040 & 0.069 & $0.021^{\circ}$ & $0.974^{\circ}$ & $0.139^{\circ}$ & $4.063^{\circ}$ & 0.994 & 2678.6 \\
\hline HEN & 0.382 & 0.008 & 32.48 & $0.133^{*}$ & $0.679^{* *}$ & 0.043 & $3.910^{\circ}$ & 0.812 & 2705.5 \\
\hline HOE & -0.396 & $0.087^{\circ}$ & 1.635 & $0.173^{*}$ & $0.761^{* *}$ & $0.160^{\circ *}$ & $4.176^{\circ}$ & 0.931 & 2674.6 \\
\hline KAR & 0.019 & $0.104^{* *}$ & $6.004^{*}$ & $0.109^{*}$ & $0.639^{* *}$ & 0.102 & $4.626^{* *}$ & 0.748 & 2519.7 \\
\hline KAU & 0.048 & 0.059 & $8.466^{\circ}$ & $0.226^{* *}$ & $0.480^{* *}$ & 0.093 & 5.01 & 0.706 & 2461.8 \\
\hline LN & -0.211 & $0.117^{* *}$ & $3.568^{* * i}$ & $0.324^{* *}$ & $0.514^{* *}$ & 0.007 & $3.720^{\circ}$ & 0.838 & 2747.3 \\
\hline LUF & -0.457 & -0.004 & 0.349 & $0.014^{\circ}$ & $0.980^{* *}$ & $0.115^{\circ}$ & “" & 94 & 2263.9 \\
\hline MAN & 0.104 & $0.088^{*}$ & 1.223 & $0.083^{\circ}$ & $0.884^{* *}$ & $0.191^{* *}$ & "'m & 0.967 & 2452.6 \\
\hline$M M N$ & 0.227 & 0.070 & 0.001 & 0.011 & $0.987^{* *}$ & $0.218^{* *}$ & $3.996^{* *}$ & 0.997 & 2403.8 \\
\hline MET & -0.413 & $0.080^{\circ}$ & 7.450 & $0.173^{* *}$ & $0.594^{* *}$ & 0.037 & 4.3 & 0.767 & 2417.5 \\
\hline PRE. & 0.281 & $0.083^{\circ}$ & 0.720 & 0.067 & $0.915^{* *}$ & 0.1 & 4.2 & 0.982 & 2412.3 \\
\hline RWE & 0.077 & 0.050 & 0.049 & 0.022 & $0.973^{\circ * x}$ & $0.143^{* *}$ & 3.7 & 0.995 & 2587.1 \\
\hline $5 \mathrm{CH}$ & -0.162 & 0.072 & 0.010 & 0.2 & 0.106 & $1.150^{\circ}$ & 4.3 & 0.313 & 2788.7 \\
\hline SIE & 0.911 & -0.006 & 0.054 & $0.029^{* 2}$ & $0.965^{* *}$ & 0.094 & $5.333^{* *}$ & 0.994 & 2680.7 \\
\hline THY & 0.429 & 0.032 & 0.265 & $0.039^{* *}$ & $0.952^{* *}$ & $0.178^{\circ}$ & $5.283^{*}$ & 0.991 & 2437.4 \\
\hline VEE & 0.254 & 0.023 & 0.029 & 0.022 & $0.974^{* *}$ & $0.170^{\circ}$ & $3.942^{\circ *}$ & 0.996 & 2592.4 \\
\hline VIA & 0.115 & 0.012 & 0.004 & 0.010 & $0.987^{* *}$ & 0.109 & $3.638^{* *}$ & 0.997 & 2556.0 \\
\hline W & 0.479 & 0.035 & 0.643 & 0.033 & $0.945^{\circ *}$ & 0.098 & $5.077^{\circ}$ & 0.978 & 2365.4 \\
\hline Median & 0.087 & 0.043 & 0.452 & 0.057 & 0.920 & 0.134 & 4.294 & 0.981 & - \\
\hline
\end{tabular}

See equations $4.23-4.28$ for a description of the parameters presented above; $\gamma$ represents the volume parameter, $L$ represents the degrees of freedom parameter and $L L$ denotes the Log-Likelihood value.

The degrees of freedom parameter in the GARCH $t$-model is significant in every case again. The empirical results so far lead to the conclusion that both volume and the number of transactions can serve as a proxy for the unobservable flow of information. Likelihood Ratio tests furthermore clearly indicate that the student $t$-distribution for conditional errors provides a better fit than the normal distribution. In the next section we will present results for the multiplicative GARCH model propose earlier. We will report results for the GARCH( 1,1$)$ tdistribution models including daily trading volume ${ }^{16}$.

16 The estimation results in Tables 4.2-4.7 clearly show that daily trading volume and the daily number of transactions provide similar results. This of course could be expected because of the large correlation between both proxies, see Table 4.1. For reasons of space and in order to protect the reader from an overkill of estimation results, we decided to choose trading volume as the proxy under at-distribution of conditional errors. 
Table 4.9 GARCH t-distribution + Contemp. Volume Estmates (Multiplicative)

\begin{tabular}{|c|c|c|c|c|c|c|c|c|c|}
\hline & $\varphi_{0}\left(10^{3}\right)$ & $\varphi_{1}$ & $a_{0}\left(10^{3}\right)$ & $\alpha_{1}$ & $\beta_{1}$ & Y. & $u$ & $\alpha_{8}+\beta_{3}$ & $\mathrm{LL}$ \\
\hline ALL & $-0.705^{\circ}$ & 0.031 & 0.958 & $0.098^{* *}$ & $0.904^{\prime *}$ & $0.640^{\circ}$ & $8.511^{* 0}$ & 1.002 & 2638.2 \\
\hline BAS & $-0.851^{\prime \prime}$ & -0.032 & 4739 & $0.109^{\circ}$ & $0.867^{\prime \prime}$ & $0.727^{\circ}$ & $13.317^{\circ}$ & 0.976 & 2760.7 \\
\hline$B A Y$ & $-0.662^{\circ}$ & -0.058 & 1.814 & $0.121^{\text {sit }}$ & $0.876^{*}$ & $0.731^{\circ}$ & $13.369^{\circ}$ & 0.997 & 2749.9 \\
\hline HYP & -0.545 & -0.013 & 0.632 & 0.048 & $0.949^{\prime \prime x}$ & $0.440^{\circ}$ & $6.203^{* 0}$ & 0.997 & 2636.7 \\
\hline VER & -0.693 & -0.031 & 1.740 & 0.082 & $0.915^{\text {"** }}$ & $0.475^{\circ}$ & $6.381^{\circ}$ & 0.997 & 2575.4 \\
\hline BMW & $-0.973^{\prime \prime}$ & 0.010 & $5279^{\circ}$ & $0.137^{*}$ & $0.850^{40}$ & $0.631^{\circ}$ & $8.532^{\text {*x }}$ & 0.987 & 2637.5 \\
\hline$C B K$ & $-1.083^{t+3}$ & -0.058 & 2.063 & $0.077^{\circ}$ & $0.916^{* *}$ & $0.603^{\circ *}$ & $10.450^{\prime *}$ & 0.993 & 26413 \\
\hline CON & $-1.485^{* .8}$ & -0.011 & 6.979 & $0.077^{* 4}$ & $0.9111^{\text {in }}$ & $0.458^{* *}$ & $8.111^{\circ}$ & 0.988 & 2374.0 \\
\hline DAl & $-1.153^{n: x}$ & -0.025 & 0.645 & $0.087^{*}$ & $0.913^{\circ \prime}$ & $0.728^{\circ}$ & $10.270^{* *}$ & 1.000 & 2607.4 \\
\hline $\mathrm{DEG}$ & $-0.987^{* *}$ & 0.0115 & $13.550^{\circ}$ & $0.078^{\circ}$ & $0.869^{\prime \prime \prime}$ & $0.490^{\circ *}$ & $5.287^{* *}$ & 0.947 & 2562.8 \\
\hline BAB & $-1.863^{6 \cdot i n}$ & -0.032 & 42.532 & $0.088^{\circ}$ & $0.827^{*}$ & $0.594^{* *}$ & $71^{* x}$ & 0.915 & 2321.6 \\
\hline DBK & $-0.663^{*}$ & -0.031 & 0.871 & $0.082^{\circ}$ & $0.915^{\circ *}$ & $0.700^{\circ}$ & $9.544^{* 4}$ & 0.997 & 2718.7 \\
\hline$D R B$ & $-0.877^{a+k}$ & 0.008 & 7.718 & 0.084 & $0.872^{\circ}$ & $0.636^{\circ}$ & $9.248^{* 4}$ & 0.956 & 2742.7 \\
\hline HEN & -0.268 & -0.051 & 2.729 & 0.047 & $0.936^{\mathrm{m}=}$ & $0.451^{\circ}$ & $4.667^{* 4}$ & 0.983 & 2741.1 \\
\hline HOE & $-0.959^{\text {:ix }}$ & -0.006 & 5.458 & $0.089^{\circ}$ & $0.884^{* *}$ & $0.601^{*}$ & $7338^{4 x}$ & 0.973 & 27264 \\
\hline KAR & -0.561 & 0.045 & 5.075 & $0.075^{\circ}$ & $0.906^{* *}$ & $0.558^{* \prime}$ & $7.044^{* t}$ & 0.981 & 2569.7 \\
\hline KAU & $-0.801^{\circ}$ & -0.012 & 12.160 & $0.107^{*}$ & $0.853^{* *}$ & $0.550^{\circ}$ & $7.981^{\text {th }}$ & 0.960 & 2515.6 \\
\hline LIN & $-0.658^{*}$ & 0.064 & 6.502 & $0.163^{*}$ & $0.810^{\circ *}$ & $0.630^{* *}$ & $3^{m * n}$ & 0.973 & 2804.4 \\
\hline LUF & $-1.761^{\text {tan }}$ & .0 .023 & $18.420^{\circ}$ & $0.079^{\circ}$ & $0.886^{*}$ & $0.545^{\circ}$ & $38^{m * t}$ & 0.964 & 2332.9 \\
\hline MAN & $-0.956^{\circ}$ & 0.055 & 6.507 & $0.086^{\prime \prime}$ & $0.895^{\mathrm{*2}}$ & $0.676^{* *}$ & $76^{i: n}$ & 0.981 & 251.4 .6 \\
\hline MMN & $-0.927^{*}$ & 0.017 & 4.205 & 0.050 & $0.936^{* *}$ & $0.762^{* *}$ & $7.767^{\text {m.* }}$ & 0.985 & 2474.2 \\
\hline MET & $-1.154^{\circ}$ & 0.013 & 15.97 & $0.100^{\circ}$ & $0.853^{* *}$ & $0.444^{* *}$ & $6.139^{\text {11** }}$ & 0.953 & 2457.9 \\
\hline PRE & $-0.9111^{\circ}$ & 0.011 & 5.937 & $0.099^{\circ *}$ & $0.890^{\circ-2}$ & $0.54 \|^{\text {i }}$ & $5.564^{4 .}$ & 0.989 & 2466.2 \\
\hline RWE & -0.576 & .0 .027 & 0.001 & $0.082^{\text {"ne }}$ & $0.913^{\circ}$ & $0.645^{10 *}$ & $6.735^{* * *}$ & 0.995 & 2662.1 \\
\hline $\mathrm{SCH}$ & -0.162 & 0.072 & 0.010 & $0.207^{* *}$ & 0.106 & $1.150^{\circ}$ & $4.347^{*}$ & 0.313 & 2788.1 \\
\hline SIE & $-0.795^{\circ}$ & $-0.061^{\prime \prime}$ & 0.631 & $0.089^{\prime \prime}$ & $0.910^{* *}$ & 0.794 & $10.280^{\circ+}$ & 0.999 & 2755.3 \\
\hline THY & $.0 .792^{\circ}$ & -0.037 & $4.278^{\circ}$ & $0.089^{* * 4}$ & $0.898^{* *}$ & $0.758^{\mathrm{ma}}$ & 24.580 & 0.988 & 2518.9 \\
\hline VEB & -0.540 & 0.004 & 0.838 & $0.072^{*}$ & $0.926^{\circ}$ & $0.708^{4.4}$ & $6.629^{* *}$ & 0.998 & 2659.1 \\
\hline VIA & $-0.795^{\circ}$ & -0.044 & 3.313 & 0.083 & $0.905^{\circ}$ & $0.678^{\prime \prime *}$ & $5.978^{k *}$ & 0.988 & 2626.2 \\
\hline WW & $-1.382^{*}$ & -0.023 & 3.705 & $0.094^{* 4}$ & $0.899^{\prime \prime}$ & $0.714^{a t}$ & $8.513^{\circ}$ & 0.993 & 2430.3 \\
\hline Median & .0 .826 & -0.013 & 4.242 & 0.087 & 0.899 & 0.634 & 7. 191 & 0.988 & $m$ \\
\hline
\end{tabular}

See equations $4.23-4.28$ for a description of the parameters presented above; $y$ represents the volume parameter, $u$ represents the degrees of freedom parameter and $L L$ denotes the Log-Likelihood value.

In the previous section we mentioned the possibility of a simultaneity bias in the additive specification. In the next section we circumvent this problem by estimating a multiplicative relation between volatility and trading volume in a GARCH context. 
Table 4.10 EGARCH Normal + Contemporaneous Volume Estimates (Multiplicative)

\begin{tabular}{|c|c|c|c|c|c|c|c|}
\hline & $\omega$ & $\Phi$ & $\delta$ & $\beta$ & 4 & $\varphi / 0$ & $L$ \\
\hline$\overline{\mathrm{ALL}}$ & -2.780 & -0.069 & $0.497^{* \circ}$ & $0.664^{* *}$ & $0.582^{\circ}$ & -0.139 & 2547.8 \\
\hline BAS & $-0.794^{* *}$ & $-0.085^{\circ}$ & $0.275^{* *}$ & $0.910^{* \circ}$ & $0.814^{6 *}$ & -0.311 & 2739.3 \\
\hline$B A Y$ & $-0.437^{x i}$ & $-0.075^{n}$ & $0.335^{* *}$ & $0.950^{\circ}$ & $0.774^{\circ *}$ & -0.224 & 2729.1 \\
\hline HYP & -1.775 & -0.010 & $0.469^{\prime *}$ & $0.784^{* *}$ & $0.485^{\circ \alpha}$ & .0 .021 & 2540.8 \\
\hline VER & $-1.166^{* 4}$ & -0.058 & $0.547^{m *}$ & $0.857^{\circ *}$ & $0.457^{* *}$ & -0.105 & 2519.0 \\
\hline BMW & $-0.380^{* *}$ & $-0.101^{\circ}$ & $0.239^{\circ *}$ & $0.955^{\circ}$ & $0.688^{\prime \prime \prime}$ & -0.421 & 2598.6 \\
\hline CBK & $-0.753^{* *}$ & -0.048 & $0.232^{\prime \prime}$ & $0.911^{* *}$ & $0.638^{24}$ & -0.205 & 2585.5 \\
\hline $\mathrm{CON}$ & -1.776 & $-0.126^{*}$ & $0.200^{* *}$ & $0.773^{* *}$ & $0.323^{11 *}$ & -0.631 & 2312.9 \\
\hline DAI & $-0.095^{\circ}$ & $-0.058^{* t}$ & $0.162^{*}$ & $0.989^{* \pi}$ & $0.775^{\text {in }}$ & .0 .360 & 2560.4 \\
\hline DEG & $-1.623^{*}$ & -0.201 & $0.417^{\mathrm{m} *}$ & $0.800^{\circ *}$ & $0.503^{* *}$ & -0.482 & 2470,6 \\
\hline $\mathrm{BAB}$ & $-1.800^{\circ}$ & $-0.165^{\prime \prime}$ & $0.164^{*}$ & $0.762^{*}$ & $0.666^{\circ *}$ & -1.010 & 2239.4 \\
\hline DBK & $-0.318^{* *}$ & -0.018 & $0.230^{* 1}$ & $0.963^{\circ *}$ & $0.807^{* *}$ & -0.080 & 2670.0 \\
\hline DRB & $-1.395^{* *}$ & -0.084 & $0.268^{* *}$ & $0.839^{\prime \prime}$ & $0.702^{* *}$ & -0.312 & 2709.2 \\
\hline HEN & -3.166 & -0.096 & $0.253^{\prime \prime}$ & $0.632^{* *}$ & $0.475^{* *}$ & -0.378 & 2652.4 \\
\hline HOE & $-1.432^{\circ}$ & -0.048 & $0.338^{\mathrm{mo}}$ & $0.833^{\circ *}$ & $0.611^{\circ}$ & -0.142 & 2682.4 \\
\hline KAR & $-0.365^{\circ}$ & $-0.104^{\circ}$ & $0.150^{\prime \prime 2}$ & $0.956^{\circ}$ & $0.657^{\circ}$ & -0.716 & 2539.3 \\
\hline KAU & $-1.087^{\circ}$ & -0.079 & $0.322^{\circ \prime}$ & $0.867^{\circ *}$ & $0.555^{\prime \prime \prime}$ & -0.245 & 2485.4 \\
\hline LIN & -0.750 & -0.006 & $0.323^{\circ}$ & $0.912^{\circ}$ & $0.787^{*}$ & -0.018 & 2715.6 \\
\hline LUF & $-1.131^{\circ}$ & $-0.116^{\circ}$ & 0.107 & $0.853^{\circ}$ & $0.440^{\circ}$ & -1.082 & 2268.6 \\
\hline MAN & -1.612 & -0.038 & $0.256^{\circ \circ}$ & $0.799^{*}$ & $0.702^{* *}$ & -0.148 & 2421.9 \\
\hline MMN & $-1.222^{\circ}$ & $-0.187^{\circ}$ & $0.157^{* \circ}$ & $0.851^{* \prime}$ & $0.842^{* *}$ & -1.186 & 2439.5 \\
\hline $\mathrm{MET}$ & $-2.985^{\circ}$ & -0.172 & 0.133 & $0.622^{\circ}$ & $0.406^{n+1}$ & -1.291 & 2334.1 \\
\hline PRE & $-2.011^{* 0}$ & $-0.167^{\circ}$ & $0.299^{\circ}$ & $0.750^{* *}$ & $0.462^{* 12}$ & -0.559 & 2409.4 \\
\hline RWE & $-0.230^{\circ}$ & $-0.097^{\circ}$ & $0.276^{*}$ & $0.973^{\circ}$ & $0.784^{n \cdot 4}$ & -0.351 & 2614.2 \\
\hline $\mathrm{SCH}$ & $-0.688^{\prime}$ & -0.051 & $0.276^{* *}$ & $0.922^{\circ}$ & $0.5722^{\cdots-2}$ & -0.186 & 27649 \\
\hline $5 \mathrm{IE}$ & $-0.468^{\circ}$ & -0.050 & $0.322^{\circ}$ & $0.946^{* *}$ & $0.868^{\prime \prime *}$ & -0.156 & 2705.2 \\
\hline THY & $-0.722^{*}$ & $-0.104^{*}$ & $0.246^{\circ}$ & $0.913^{* *}$ & 0.743 & -0.421 & 2477.9 \\
\hline VEB & $-0.499^{\circ}$ & $-0.112^{\circ}$ & $0.327^{*+}$ & $0.940^{* *}$ & $0.855^{k+4}$ & -0.341 & 2595.4 \\
\hline VIA & $-1.046^{\circ *}$ & $-0.217^{* *}$ & $0.403^{* *}$ & $0.877^{*}$ & $0.799^{\circ .4}$ & -0.538 & 2569.9 \\
\hline W & $.0 .451^{\circ}$ & $-0.117^{* *}$ & $0.165^{* *}$ & $0.944^{* *}$ & $0.758^{\circ *}$ & -0.710 & 2354.3 \\
\hline
\end{tabular}

See equations 4.30 and 4.31 for a detailed description of the parameters presented above; $y$ represents the volume parameter, $L$ denotes the Log-Likelihood value.

\subsection{A Multiplicative Specification}

Table 4.8 contains estimation results of the multiplicative specification as in equation 4.28 including lagged daily trading volume, thereby circumventing the simultaneity problem of the additive model ${ }^{17}$. Rather interestingly, here GARCH-parameters remain significant and do not drop in value in this setting. As we expected, the volume parameter $\gamma$ is statistically significant in most cases 4.20 , which indicates that trading volume can be interpreted as a time-varying scale factor for conditional volatility.

17 Values of trading volume and the number of transactions are divided by their mean in order to scale them before estimating the multiplicative specification. 
Table 4.11 GARCH Restricted Modele Is -distribution Estimates

\begin{tabular}{lccccc}
\hline & $\varphi_{0}(10)^{3}$ & $\alpha_{0}$ & $\alpha_{0}(10)^{3}$ & $\gamma$ & $v$ \\
\hline Median (LM) & -0.021 & 0.039 & 0.281 & 0.144 & 3.588 \\
Median (CM) & -0.727 & -0.001 & 0.256 & 0.460 & 4.735 \\
\hline
\end{tabular}

Vepresents the trading volume parameter, whe degrees of freedom parameter, $L M$ denotes the lagged model and CM denotes the contemporanecus model.

Table 4.12 Diagnostics GARCH Multiplicative Model Contemporaneous Volume

\begin{tabular}{ll}
\hline Statistic & Cross-Security Medians \\
\hline Skewness & -0.56 \\
Kurtosis & 8.49 \\
$\mathbb{F}(1)$ & 2.75 \\
$\mathbb{F}(2)$ & 2.53 \\
$F(5)$ & 1.27 \\
\hline
\end{tabular}

For an explanation of the F-test, see text and Chapter 3.

A direct comparison of the additive L\&L model with contemporaneous volume and our multiplicative model with lagged volume is not possible ${ }^{18}$. If we replace lagged volume by contemporaneous volume in equation 4.28 , see Table 4.9 , we find $\gamma$ to be significant in every single case. The GARCH parameters remain significant again, which gives reason to believe that volume plays an important role over and above its function as a possible proxy for information arrival.

Results for the multiplicative EGARCH models including contemporaneous volume are contained in Table 4.10 . In general there appears to be a very mild asymmetry or leverage effect as the relevant parameters $\varphi$ and $\delta$ are both significant at the $5 \%$ level in only 13 cases. The volume parameter remains significant. In Table 4.11 we report median estimates of the restricted versions $\left(\alpha_{1}=\beta_{1}=0\right)$ of the lagged and contemporaneous specification. The volume parameter is significant in almost every case in the lagged case and for every single stock in the contemporaneous case. It should be noted that all models have also been estimated including the daily number of transactions instead of daily trading volume. Results were approximately the same for every specification estimated. Casual inspection of the log-likelihoods reveals that the multiplicative GARCH model using contemporaneous trading volume provides the best fit ${ }^{19}$.

18 It should be noted here that estimating the additive specification with lagged trading volumes did not provide one significant estimate for the volume parameter. This is consistent with the results of Lamoureux and Lastrapes (1990a).

19 Formal testing using a likelihood ratio test is however not possible as most models are non-nested. For reasons of space we decided to subject the model with the highest likelihood values to diagnostic checks, i.e. the GARCH multiplicative model with contemporaneous trading volumes. 
Table 4.13 Weekly Aggregated Volume Median Estimates

\begin{tabular}{lllll}
\hline & $\alpha_{1}$ & $\beta_{1}$ & $\gamma$ & $\alpha_{1}+\beta_{1}$ \\
\hline AM & 0.083 & 0.000 & 1.199 & 0.095 \\
MM & 0.158 & 0.782 & 0.688 & 0.923 \\
\hline
\end{tabular}

AM denotes additive model and MM denotes multiplicative model.

In order to determine the adequacy of this specification, the model is subjected to diagnostic checks on the standardized residuals. Results presented in Table 4.12 show that there is a much less consistent rejection of the normality hypothesis as compared to the results presented in Table 4.1. In particular, the crosssecurity median skewness and kurtosis values drop to -0.56 and 8.49 respectively. In almost all individual cases, skewness and kurtosis values support our model specification. In order to test for remaining heteroskedasticity, a residualbased test of this model is carried out by regressing squared estimated residuals on one to five lags ${ }^{20}$. The estimated coefficients of this regression are then subjected to a convential F-test, see Domowitz and Hakkio (1985) and Pagan and Hall (1983). The results are reported under $F(1), F(2)$ and $F(5)$. These statistics follow $\mathrm{F}(2,881), \mathrm{F}(3,879)$ and $\mathrm{F}(6,873)$ distributions respectively. Median values of this test statistics are all statistically insignificant at the 5\% level, implying that there is no heteroskedasticity left in the residuals.

\subsection{Lower Frequency (Weekly) Results}

Estimation results so far were conducted on a sample of daily returns and daily trading volumes. In Chapter 3 we saw that GARCH effects are often present in lower frequency stock (index) returns too. We therefore would like to know whether the multiplicative model we present in section 4.3 gives significant results in a weekly context $t^{21}$. We are particularly interested in the question whether the relationship between volatility and trading volume is less strong in the lower frequency case. Moreover we would like to know whether the same differences appear between additive and multiplicative model results respectively. In these models the distribution of intra-weekly price increments and the distribution of the weekly rate of information arrival are mixed. We use the additive specification in equation 4.13 and the multiplicative specification in equation 4.28 , both under a normal distribution of conditional error terms ${ }^{22}$. The

20 We introduce this test statistic in Chapter 3.

2! In Chapter 3 we saw that GARCH effects tend to fade away in many cases when using monthly frequencies. We therefore decided to use weekly aggregated volumes and weekly returns.

22 We also estimated this model under a student $t$-distribution, but Log-Likelihood values did not improve and the value of the degree of freedom parameter $v$ indicated a normal distri- 
sample of weekly returns and weekly aggregated trading volumes contains 178 observations, where the period under investigation remains the same. In Table 4.13 estimation results for this specification are reported ${ }^{23}$.

The results in Table 4.13 clearly show that the relationship between volatility and trading volume also exists in a weekly context. In the additive specification, consistent with earlier results, the sum of the GARCH parameters in the lower frequency case is very low. In the multiplicative specification however, the persistence in volatility remains high despite the inclusion of trading volume. The volume parameter is significant in 20 cases in the additive model and in every single case in the multiplicative model. These results on the one hand indicate that the research into the relationship between volatility and trading volume on stock markets should not be restricted to just intra-daily and daily samples. On the other hand Table 4.13 provides evidence that the results for daily stock returns are also valid in a lower frequency environment.

\subsection{Interpretation of Results and Concluding Comments}

We have shown that the characteristics of daily German stock returns and the corresponding daily trading volume and number of transactions, generally confirm the predictions generated by the mixture of distributions hypothesis. In order to provide some intuition into the sources of GARCH we used a pragmatic version of the MDH as a starting point in our discussion. In particular, we tried to explain why previous studies used daily trading volume or the number of transactions in a GARCH process as a proxy for the rate of information arrival. We have demonstrated that the MDH related framework of L\&L has several drawbacks, such as an unobservable mixing variable, a single source of conditional heteroskedasticity, a questionable move from theory to a testable specification, and a possible simultaneity bias in the resulting specification.

We contribute to the existing literature by integrating the unobservable mixing variable out of the problem, allowing for multiple sources of conditional heteroskedasticity, and reinterpreting trading volume as a time-varying scale factor for conditional volatility, thereby removing the possibility of a simultaneity bias. Empirical results confirm our expectation that there is a strong link between trading volume and stock return volatility. The major empirical result

23

bution.

3 For reasons of space and simplicity we decided to report median estimates. Individual results are available upon request. In addition we estimated a lagged specification, but as we expected the relationship between lagged weekly trading volume and weekly volatility does not exist. 
in this paper is that daily trading volume and the daily number of transactions have explanatory power over and above their use as a proxy for information arrival, and can be used as a scale factor for volatility. An intuitive and appealing explanation for this phenomenon could be that trading volume and the number of trades display a measure of diversity of interpretation of information, or alternatively a measure of disagreement about received information among investors in the German stock market. Disagreement among traders and investors possibly leads to more trading activity in the stock market and maybe to more uncertainty among traders. Therefore, incorporating daily trading volume as a time-varying scale factor for volatility possibly accounts for this phenomenon. In addition, we find that the persistence in volatility does not drop in value in the multiplicative specification, whereas our additive specification results show a considerable drop in persistence.

Useful suggestions for further research are the incorporation of alternative distributions for the mixing variable. Moreover, one could think of alternative additive specifications where volume's exponent is different from one. In this chapter we included trading volume as a time-varying scale factor in a univariate GARCH model. It should be noted that the standard mixture hypothesis of Clark (1973) states that both price changes and trading volume are governed by the same latent mixing variable simultaneously. Therefore a bivariate modelling approach with prices and trading volume both influenced by the information process could be more appropriate. In this setting trading volume does not have a role as a proxy for information arrival, but is in fact endogenized as in the Lamoureux and Lastrapes (1994) paper. This bivariate modelling framework is economically more appealing and furthermore in line with the standard mixture hypothesis. Chapters 6 and 7 will therefore concentrate on these models in greater detail. In the next chapter we however first present the univariate approach shown in this chapter in a stochastic volatility framework and subsequently compare it with our univariate GARCH results.

\section{Appendix A Derivation of the Multiplicative GARCH Model}

As in Koedijk, Nissen, Schotman en Wolff (1994) we use an AR(1) in the mean specification, assuming conditional volatility of the following form:

$$
\begin{array}{lcc}
R_{t}=\varphi_{0}+\varphi_{1} R_{t-1}+e_{t}, \quad e_{t} \mid\left(V_{t}, e_{t-1}, e_{t-2}, \ldots\right) \sim t\left(0, h_{t}, v\right) \\
\sqrt{h_{t}}=\sigma_{t} V_{t-1}^{\gamma}, \quad \text { so } \quad h_{t}=\sigma_{t}^{2} V_{t-1}^{2 \gamma}
\end{array}
$$

The prediction error $e_{i}$ is defined as: 
$e_{t}=\sqrt{h_{t}} \varepsilon_{t}, \quad \varepsilon_{t} \sim t(0,1, v)$

Substituting for $h_{i}$ yields:

$e_{t}=\sigma_{t} V_{t-1}^{\gamma} \varepsilon_{t}$

We now assume that $\sigma_{t}$ is generated by a $\operatorname{GARCH}(1,1)$ process:

$\sigma_{t}^{2}=\alpha_{0}+\alpha_{1}\left(\varepsilon_{t-1}^{*}\right)^{2}+\beta_{1}\left(\sigma_{t-1}\right)^{2}$

where:

$\varepsilon_{t-1}^{\prime \prime}=\sigma_{t-1} \varepsilon_{t-1}$

and subsequently:

$$
\varepsilon_{t-1}^{*}=\frac{e_{t-1}}{V_{t-2}^{\gamma}} \Rightarrow\left(\varepsilon_{t-1}^{*}\right)^{2}=\frac{e_{t-1}^{2}}{V_{t-2}^{2 \gamma}}
$$

If we now substitute $\mathrm{A} 5$ in $\mathrm{A} 2$ and solve for $\sigma_{t}$ we get:

$h_{t}=\alpha_{0} V_{t-1}^{2 \gamma}+\alpha_{1}\left(\varepsilon_{t-1}^{*}\right)^{2} V_{t-1}^{2 \gamma}+\left(\frac{V_{t-1}}{V_{t-2}}\right)^{2 \gamma}\left(\beta_{1} h_{t-1}\right)$

Substituting A7 in A8 leads to the following specification for the conditional variance:

$h_{i}=\alpha_{0} V_{t-1}^{2 \gamma}+\left(\frac{V_{t-1}}{V_{t-2}}\right)^{2 \gamma}\left(\alpha_{1} e_{t-1}^{2}+\beta_{1} h_{t-1}\right)$

If we alternatively assume conditional volatility of the form:

$\sqrt{h_{t}}=\sigma_{i} V_{t}^{\gamma}$

A9 becomes:

$h_{t}=\alpha_{0} V_{t}^{2 \gamma}+\left(\frac{V_{t}}{V_{t-1}}\right)^{2 \gamma}\left(\alpha_{1} e_{t-1}^{2}+\beta_{1} h_{t-1}^{2}\right)$

Both $\mathrm{A} 9$ and $\mathrm{A} 11$ are estimated in the empirical section. 


\section{Appendix B Integrating out the Mixing Variable}

We know that:

$p\left(\varepsilon_{t} \mid \sqrt{n_{t}}, \Psi_{t-1}\right)=\frac{1}{\sqrt{2 \pi} \sigma_{t} \sqrt{n_{t}}} \exp \left(-\frac{\varepsilon_{t}^{2}}{2 \sigma_{t}^{2} n_{t}}\right)$

We assume:

$p\left(\sqrt{n_{t}}\right)=\frac{2}{\Gamma(\alpha) \gamma^{\alpha} n_{t}{ }^{\alpha+0.5}} \exp \left(-\frac{1}{\gamma n_{t}}\right)$

Now, we make the following change of parameters:

$\alpha=v / 2 \quad, \quad \gamma=2 /(v \beta)$

This leads to:

$p\left(\sqrt{n_{t}}\right)=\frac{2}{\Gamma\left(\frac{v}{2}\right)}\left(\frac{v \beta}{2}\right)^{\frac{v}{2}} \frac{1}{\left(\sqrt{n_{t}}\right)^{p+1}} \exp \left(\frac{-v \beta}{2 n_{t}}\right)$

The joint distribution, which can be obtained by multiplying the conditional distribution in B1 by the marginal distribution in B4, becomes:

$p\left(\varepsilon_{t} \sqrt{n_{t}} \mid \Psi_{t-1}\right)=\frac{\sqrt{2}}{\Gamma\left(\frac{1}{2}\right) \Gamma\left(\frac{v}{2}\right) \sigma_{t}}\left(\frac{v \beta}{2}\right)^{\frac{\nu}{2}} \frac{1}{\left(\sqrt{n_{t}}\right)^{0+2}} \exp \left(-\frac{1}{2}\left(\frac{v \beta}{n_{t}}+\frac{\varepsilon_{t}^{2}}{\sigma_{t}^{2} n_{t}}\right)\right)$

Now, consider the following changes in variables and parameters:

$c=\frac{\sqrt{2}}{\Gamma\left(\frac{1}{2}\right) \Gamma\left(\frac{v}{2}\right) \sigma_{t}}\left(\frac{v \beta}{2}\right)^{\frac{v}{2}}, \quad y=\sqrt{n_{t}}, \quad a=\frac{\varepsilon_{t}^{2}}{\sigma_{t}^{2}}+v \beta$

The distribution of the innovation terms conditional on the information set can now be solved from:

$p\left(\varepsilon_{t} \mid \Psi_{t-1}\right)=c \int_{a}^{\infty} y^{-(0+2)} \exp \left(-\frac{a}{2 y^{2}}\right) d y$

With another change of variable: $x=a / 2 y^{2}$, we obtain:

$p\left(\varepsilon_{t} \mid \Psi_{t-1}\right)=c a^{\left(-\frac{v+1}{2}\right)_{2}\left(\frac{v-1}{2}\right)} \exp (-x) d x$ 
The integrall in $B 7$ is nothing but the gamma function, so after evaluating this function, replacing the new variables by the old variables and setting $\beta=1 / \nu$, we eventually obtain:

$$
p\left(\varepsilon_{t} \mid \Psi_{t-1}\right)=\frac{\Gamma\left(\frac{v+1}{2}\right)}{\Gamma\left(\frac{1}{2}\right) \Gamma\left(\frac{v}{2}\right) \sigma_{t}}\left(1+\frac{\varepsilon_{t}^{2}}{\sigma_{t}^{2}}\right)-\left(\frac{v+1}{2}\right)
$$

This a student $t$-distribution with $v$ degrees of freedom. The distribution conditional on $h_{t}$ can be derived analogously, by replacing $\sigma^{2}$ by $h_{\text {: }}$.

\section{Appendix C List of Company Abbreviations}
ALL: Allianz
BAS: BASF
BAY: Bayer
HYP: Bayerische Hypothekbank
VER: Bayerische Vereinsbank
BMW: $\quad$ BMW
CBK: Commerzbank
CON: Continental
DAI: Daimler-Benz
DEG: Degussa
BAB: Deutsche Babcock
DBK: Deutsche Bank
DRB: Dresdner Bank
HEN: Henkel
HOE: Hoechst
KAR: Karstadt
KAU: Kaufhof
LUF: Lufthansa
LIN: Linde
MMN: Mannesmann
PRE: Preussag
SCH: Schering
THY: "Thyssen
MAN: MAN
MET: Metallgesellschaft
RWE: RWE
SIE: Siemens
VEB: VEB
VIA: VIAG
VW: Volkswagen 


\section{CHAPTER 5}

\section{Embedding Volume as a Scale Factor in a SV Model}

\subsection{Introduction}

In the last decade the econometric society has paid growing attention to the link between price changes, the volatility of these price changes and trading volume. Most of these studies build on the models presented by Clark (1973), Epps and Epps (1976) and Tauchen and Pitts (1983). The main topics of analysis are the possible non-linearities and asymmetries in financial return series and its possible relation with the amount traded on the markets concerned. As documented extensively in Chapters 3 and 4 , the structure in second order moments is captured in the GARCH literature, see Bollerslev, Chou and Kroner (1992). The asymmetric response of volatility to return shocks has been studied by Nelson (1991), who proposed the EGARCH specification. Econometric studies into the link between volatility and trading volume are offered by Gallant, Rossi and Tauchen (1992, 1993), Tauchen, Zhang and Liu (1993), LeBaron (1993), Lamoureux and Lastrapes (1990a, 1994) and Andersen (1996).

The various parametric techniques used in these studies vary from including trading volume exogenously in the conditional variance equation of a GARCH model, see Lamoureux and Lastrapes (1990a) to combining the mixture of distributions hypothesis $(\mathrm{MDH})$ with a stochastic volatility model, see Andersen (1994). In addition severable semi-nonparametric, see Gallant, Rossi and Tauchen $(1992,1993)$, and completely non-parametric approaches, see Tauchen, Zhang and Liu (1993), have been used to investigate the interrelationships between stock price volatility, trading volume and the leverage effect. The general upshot from the economic strand in the literature is that (a) the MDH at least requires some modification, (b) the leverage effect at best is present but short-lived at the individual asset level, see Gallant, Rossi and Tauchen (1993), Engle and Lee (1993), and Tauchen, Zhang and Liu (1993), and (c) trading volume possibly responds in a non-linear fashion to shocks in the price process.

In our view the role of trading volume needs more attention in this discussion. Can it be used as a proxy for information arrival, see Lamoureux and Lastrapes 
(1990a), is it possible to explain GARCH by a stochastic rate of information arrival proxied by trading volume, the number of transactions or even the daily number of price changes (see Laux and $\mathrm{Ng}$ (1993))? In this chapter we try to shed some light on these questions. Although we acknowledge the fact that possible non-linearities are present, see for instance LeBaron (1993), we first estimate some simple linear Vector AutoRegressive (VAR) models in order to capture some of the basic dependencies between stock returns, the volatility of stock returns and trading volume. Consequently, as we find no firm conclusion in a VAR context, we propose a new role for trading volume. In particular, we construct a Stochastic Volatility (SV) model in which trading volume acts as a scale factor for the conditional volatility of the stock return process. Furthermore considerable attention is given to the asymmetric effects from shocks in returns to conditional volatility. Several sign bias tests are performed in order to investigate whether there is a difference in the impact of positive and negative shocks in returns on the volatility. The SV model we use in our estimations is rearranged to capture these asymmetries. We apply Quasi Maximum Likelihood methods to estimate these models. Moreover we will estimate several other models, i.e. GARCH and EGARCH specifications. These models are equal to the models estimated in Chapter 4 and they serve as a benchmark for our new SV model specification.

Our approach is rather eclectic in the sense that parts of our analysis have appeared elsewhere, but other parts are quite novel. We will refer to links with the existing literature where appropriate, and compare our results to the findings mentioned earlier. The remainder of this Chapter is organized as follows. In section 5.2 we will introduce the concept of stochastic volatility by presenting a basic and simple stochastic variance model. In addition, we will discuss several theoretical and empirical studies in this relatively new research area. In section 5.3 we will present our new SV model which includes trading volume as a scale factor and takes into account the previously mentioned leverage effect. Furthermore we will discuss the possible estimation techniques in greater detail here. Before estimating our new SV model, we first describe our data set and present summary statistics in section 5.4. Sign bias tests are performed in section 5.5 and Granger causality tests and several linear VAR models in section 5.6. In section 5.7 we present the results of an extensive investigation into the empirical evidence of our Stochastic Volatility, GARCH- and EGARCH-models. Finally, Section 5.8 concludes and gives some suggestions for further research.

1. It should be noted that we concentrate on the link between volatility and volume. The discussion on the appropriate estimation techniques is beyond the scope of this research. 


\subsection{The Concept of Stochastic Vollatility}

In (G)ARCH models, documented extensively in the previous chapters, the changing variance is dependent on past squared residuals and past variances. An alternative way of modelling time-varying variances is presented in Taylor (1986), Harvey (1989), Harvey and Shephard (1994) and Harvey, Ruiz and Shephard (1994). In their approach, the variance is treated as an unobservable variable following a certain kind of linear stochastic process, often of an autoregressive nature. These models are known in the finance literature as stochastic volatility or stochastic variance (SV) models ${ }^{2}$. These models have the advantage that, if formulated in continuous time, they are consistent with the theoretical framework within which the valuation of all kinds of options has been investigated ${ }^{3}$. One of the other strengths of these models in relation to the GARCH class of models, is their ability to estimate multivariate series, without the unfavourable implication of the exploding number of parameters in a multivariate GARCH context. Their major disadvantage relative to GARCH models is however that it is very difficult to write down an expression for the exact likelihood function, which leads to problems in estimating the SV model. In this section we discuss the estimation of stochastic volatility models in greater detail. The basic stochastic variance model can be represented as follows:

Let us assume that the time series we are interested in, $y_{t}$, can be described by a Gaussian white noise process with unit variance $\left(\varepsilon_{l}\right)$, multiplied by the standard deviation $\left(\sigma_{t}\right)$. The following specification then arises:

$$
y_{t}=\sigma_{t} \varepsilon_{t} \quad t=1, \ldots, T, \quad \varepsilon_{t} \sim N I D(0,1)
$$

In a stochastic variance model for equation 5.1, the natural logarithm of $\sigma_{t}^{2}$, often denoted as $h_{\mathrm{f}}$ is modelled as an unobserved component that behaves stochastically ${ }^{4}$. A simple model for $h_{t}$ is the AR(1) process, see Harvey (1989) and Harvey, Ruiz and Shephard (1994). This leads to the following well-known specification for the stochastic variance:

$h_{t}=\gamma+\varphi h_{t-1}+\eta_{t}, \quad \eta_{t}-N I D\left(0, \sigma_{\eta}^{2}\right)$

2 Taylor (1994) uses the expression "autoregressive random variance model" (ARV) to describe the stochastic volatility modells.

3. See Hull and White (1987) for a generalisation of the Black and Scholes option pricing formula.

4. Working with logarithms has the advantage that volatility is always positive. For early references, see Taylor (1986). If the AR(1) parameter $\varphi<1$, we know that $h_{s}$ is strictly stationary, with mean $\gamma_{h}$ and wariance $\sigma_{k}^{2}=\sigma_{\eta}^{2} /\left(I-\varphi^{2}\right)$, see Harvey (1989). In that case, the stock return series $y_{s}$ is the product of two strictly stationary processes and therefore it must be strictlly stationary too. 
where $\eta_{i}$ is generated independently of $\varepsilon_{t}^{5}$. As we stated above, the time-varying conditional variance is the natural logarithm of the variance in 5.1 and therefore:

$$
\sigma_{t}^{2}=\exp ^{\hbar_{i_{t}}}, \sigma_{t}=\exp ^{0.5 h_{t}}
$$

Substituting for $\sigma_{t}$ in equation 5.1 yields:

$y_{t}=\exp ^{0.5 h_{t}} \varepsilon_{t}$

If we now transform the original series by squaring them and taking the natural logarithm we eventually get:

$\ln y_{i}^{2}=h_{t}+\ln \varepsilon_{t}^{2}$

If $\varepsilon_{k}$ follows a standard normal distribution, the mean and variance of the second term of the right-hand side of 5.5 are -1.27 and $\pi^{2} / 2$ (4.93) respectively. The resulting model can be presented in a linear state space format:

$\ln y_{t}^{2}=h_{t}+\xi_{t}$

$h_{t}=\gamma+\varphi h_{t-1}+\eta_{t}$

where $\xi_{t}=\ln \varepsilon_{t}^{2}$. The estimation of SV models receives considerable attention lately. Since the model above is not conditionally Gaussian, it is difficult to write down the exact likelihood in a stochastic volatility framework, which complicates the estimation procedure considerably relative to GARCH models. Formally, Maximum Likelihood (ML) estimation requires a method to find the solution of a high-dimensional integral, that arises due to the latent character of the transition (volatility) equation and the non-normality of $\xi_{i}$. Simulation studies can circumvent this problem, see Jacquier, Polson and Rossi (1994) and Kim and Shephard (1994), but these techniques are very computer intensive and rather time consuming?. The estimation of SV models has also been carried out by the Generalised Method of Moments (GMM), see for instance Chesney and Scott (1989) and Melino and Turnbull (1990). Another approach is to approximate the error distribution of $\xi_{t}$ by a normal distribution and consequently apply the usual Kalman filter techniques. The studies of Harvey and Shephard

5 Note that equation 5.2 is the natural discrete time approximation to the continuous time Ornstein-Uhlenbeck process used in finance theory, see Harvey, Ruiz and Shephard (1994).

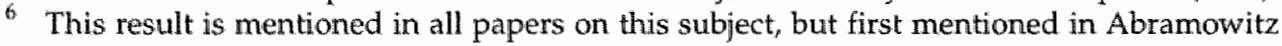
and Stegun (1970).

7 In Chapter 6 we apply a simulated maximum likelihood (SML) approach based on the work of Danielsson and Richard (1993). 
(1994), Mahieu and Schotman (1996) and Ruiz (1994) are excellent examples of estimating these models by Quasi-Maximum Likelihood (QML) based on the Kalman filter.

The results reported in Ruiz (1994) furthermore indicate that the relative efficiency of the QML estimator when compared to estimators based on the GMM is quite high. In particular, it is shown that the QML procedure functions well for sample sizes typically encountered in financial economics and is usually preferred to the GMM procedure. Although QML estimation procedures may give less efficient parameter estimates compared to exact methods, we decide to use QML as it is a simple and straightforward way of estimating the hyperparameters of a SV model. In our opinion, it at the very least is able to provide preliminary information on a suitable model specification. In the next section we will modify the state space model by including daily trading volume as a scale factor in the measurement equation. We will furthermore account for possible leverage effects by adjusting the linear state space format.

\subsection{Stochastic Volatility and Volume}

Many researchers have focused on the sources of conditional heteroskedasticity in asset markets. One of these approaches that has been studied thoroughly, mentioned in Chapter 4, is the Mixture of Distributions Hypothesis (MDH), introduced by Clark (1973) and extended by several authors. This theory explicitly states that a daily return observation is drawn from a mixture of distributions. Let $y_{t}$ be the (daily) return on a financial asset. Moreover, suppose that this return consists of a number of intra daily price increments $\delta_{i t}$ :

$y_{t}=\sum_{i=1}^{n_{t}} \delta_{i t}$

where $n_{t r}$ analogous to the model presented in Chapter 4 , is an integer variable that represents the number of price increments occurring on day $t$. The $\delta_{i t}$ are i.i.d. with mean zero and variance $\sigma^{2}$. If we consider the price increments as information events, as for example in Harris (1987), $n$, determines the rate of information arrival. In this interpretation $n$ is the stochastic mixing variable. In the current setting $n_{t}$ is the directing process for the subordinated process $y_{p}$. In other words, it governs the number of information arrivals which in turn determine daily returns. ${ }^{8}$. For example, if the number of information events is high, a lot of information pieces enter the market. Clark (1973) shows that, provided

8 For a detailed discussion on the MDH see Chapter 2. 
that the number of information arrivals is large, a central limit theorem argument can be made that results in:

$y_{p} \mid n_{t} \sim N\left(0, \partial^{2} n_{t}\right)$

As noted in Chapter 4 the conditioning variable $n_{t}$ in 5.9 is unobservable. Therefore, a suitable proxy for $n_{t}$ has to be chosen. Consistent with our approach in the previous chapter and various empirical studies, e.g. Lamoureux and Lastrapes (1990a, 1994), we will use daily trading volume as a proxy for the directing process. More specifically, we will make a direct link to the Stochastic Volatility models presented in section 5.2 . In particular, we rewrite 5.9 as:

$y_{t}=\sigma \sqrt{n_{t} \varepsilon_{t}}, \quad \quad \varepsilon_{t}-N(0,1)$

Let us in addition assume that the rate of information arrival follows an $\mathrm{AR}(1)$ process:

$n_{t}=\beta+\varphi n_{t-1}+\eta_{t} \quad \eta_{t} \sim N\left(0, a_{\eta}^{2}\right)$

Note that this is in fact a SV model in which the volatility is represented by the number of information arrivals. We furthermore need the assumption that $n_{t}$ is always positive. One way to ensure this is to let $h_{t}$ be the natural logarithm of $n_{t}$. In this case we retrieve the following lognormal SV model with autoregressive volatility:

$\begin{array}{ll}y_{t}=\sigma \varepsilon_{t} \exp \left(\frac{h_{t}}{2}\right), & \varepsilon_{t}-N(0,1) \\ h_{t}=\beta+\varphi h_{t-1}+\eta_{t}, & \eta_{t} \sim N\left(0, \sigma_{\eta}^{2}\right)\end{array}$

From 5.12 and 5.13 we see that SV models are closely linked to the MDH. The variable $h_{4}$ can either be interpreted as volatility or as a transformed information arrival process. Estimation of these models thus leads to an estimate of the information arrival process. From 5.9 we see that the number of information arrivals $n_{t}$ is multiplicatively related to the conditional variance of the $y_{*}$. 
In Chapter 4, we suggested to introduce trading volume as a scale factor for the conditional volatility. In this section we will follow the same procedure by constructing a linear state space model in which trading volume acts as a scale factor for volatility. In particular, we will estimate a stochastic volatility model in which trading volume is included in the measurement equation of the state space model. In addition, we also account for possible leverage effects by allowing correlation between measurement and transition equation disturbances.

Let $y_{t}$ be the return on a given individual stock at time $t_{n}$, the conditional volatility be represented by $h_{t}$ and the corresponding daily trading volume by $V_{t}$ then the augmented measurement equation of the basic stochastic volatility model incorporating volume as a scale factor can be given as:

$$
\begin{array}{ll}
y_{t}=\sigma \varepsilon_{t} \exp \left(\frac{h_{t}}{2}\right) V_{t}^{\lambda_{y}} & \varepsilon_{t}-\operatorname{NID}(0,1) \\
h_{t+1}=\beta+\varphi h_{t}+\eta_{t} & \eta_{t} \sim N\left(0, \sigma_{\eta}^{2}\right)
\end{array}
$$

In this model specification trading volume is included in the measurement equation as a scale factor for the conditional volatility $h_{t}$. The innovations in the measurement and transition equation are contemporaneously correlated by a parameter $\rho$. Moreover we assume that:

$$
\operatorname{Corr}\left(\varepsilon_{t}, \eta_{t-k}\right)=\operatorname{Corr}\left(\varepsilon_{t-k^{\prime}} \eta_{t}\right)=0 \quad \text { for } k \neq 0
$$

The volatility process is assumed to be $\mathrm{AR}(1)$, but of course other specifications are possible. Assuming a negative contemporaneous correlation between both innovations can be interpreted as a leverage effect: negative shocks in stock prices increase volatility more than positive shocks. Note that we shifted the volatility one period ahead in 5.15. Equations 5.14 and 5.15 can be linearized again by squaring the return series and taking logarithms. The model we use in the empirical section can then be specified in a linear state space format as follows:

$$
\begin{aligned}
& \ln y_{t}^{2}=h_{t}+\lambda_{y} \ln V_{t}+\beta_{y}+\xi_{y, t} \\
& h_{t \rightarrow 1}=\varphi h_{t}+\eta_{t}
\end{aligned}
$$

Here the constant parameter $\beta_{y}$ incorporates both the constants $\sigma$ from 5.14 and $\beta$ from 5.15 , without the loss of generality. The vector of innovations is distributed as: 


$$
\left(\begin{array}{c}
\xi_{y_{w} t} \\
\eta_{t}
\end{array}\right)=\operatorname{IID}\left(0_{n}\left[\begin{array}{cc}
\sigma_{\xi}^{2} & \rho \sigma_{\xi} \sigma_{n} \\
\rho \sigma_{\xi} \sigma_{n} & \sigma_{\eta}^{2}
\end{array}\right]\right)
$$

Note that trading volume is included in the measurement equation 5.17 now. The state space model presented above cannot be estimated via the well-known Kalman filter technique directly, since inference about the correlation parameter is invalid. The problem is that by squaring observations, valuable information about the sign of the correlation coefficient is lost. Harvey and Shephard (1994) (HS94) develop a method to estimate $\rho$ within the state space formulation. They show that the sign of the observations are ancillary statistics. This implies that we can condition on the signs in order to find an estimate for $\rho$. We follow the HS94 approach and find the following linear state space model\%:

$$
\begin{aligned}
& \ln y_{t}^{2}=h_{t}+\lambda_{y} \ln V_{t}+\beta_{y}+\xi_{y, t} \\
& h_{t+1}=\varphi h_{t}+s_{t} k+\eta_{t}^{*} \\
& \left(\begin{array}{c}
\xi_{y_{t} t} \\
\eta_{t}^{*}
\end{array}\right) \mid s_{t}=I I D\left(0,\left[\begin{array}{cc}
\sigma_{\xi}^{2} & v s_{t} \\
v s_{t} & \sigma_{\eta}^{2}-k^{2}
\end{array}\right]\right)
\end{aligned}
$$

where $\nu=\operatorname{Cov}_{*}\left(\eta_{i}, \xi_{i}\right)$, is the conditional covariance where we condition on positive observations. The measurement equation does not change when compared to 5.17 , but the new transition equation is now dependent on the sign of the observations:

$s_{t}= \begin{cases}1 & \text { if } y_{t}>0 \\ -1 & \text { if } y_{t} \leq 0\end{cases}$

Define $k$ to be the expectation of $\eta_{t}$ conditional on $s_{f}$ :

$$
k=E\left(\eta_{t} \mid s_{t}=1\right)=-E\left(\eta_{t} \mid s_{t}=-1\right)
$$

Harvey and Shephard (1994) introduced this model, however without incorporating trading volume as a scale factor for volatility. 
The last equivalence follows since the joint distribution of the error terms, see Harvey, Ruiz and Shephard (1994) for further details; is assumed to be symmetric. Using the ancillarity features of the sign parameters $s_{t}$ we eventually obtain the following state space model:

$$
\begin{aligned}
& \ln y_{t}^{2}=h_{t}+\lambda_{y} \ln V_{t}+\beta_{y}+\xi_{y, t} \\
& h_{t+1}=\left(\varphi-\frac{v s_{t}}{\sigma_{\xi}^{2}}\right) h_{t}+s_{t}\left(k+\frac{v}{\sigma_{\xi}^{2}}\left(\ln y_{t}^{2}-\lambda_{y} \ln V_{t}-\beta_{y}\right)\right)+\eta_{t}^{*}
\end{aligned}
$$

with the following disturbances:

$$
\left(\begin{array}{c}
\xi_{y, t} \\
\eta_{t}^{*}
\end{array}\right)=\operatorname{IID}\left(0,\left[\begin{array}{cc}
\sigma_{\xi}^{2} & 0 \\
0 & \sigma_{\eta}^{2}-k^{2}-\frac{v^{2}}{\sigma_{\xi}^{2}}
\end{array}\right]\right)
$$

Analogous to HS94 we assume that $\mathcal{E}_{t}$ and $\eta_{t}$ are bivariate normal with correlation parameter $\rho$. This means that:

$$
\begin{aligned}
& k=E\left[\eta_{t} \mid s_{t}=1\right]=-E\left[\eta_{t} \mid s_{t}=-1\right]=0.7979 \rho \sigma_{\eta} \\
& v=\operatorname{Cov}\left[\eta_{t}, \xi_{t} \mid s_{t}=1\right]=-\operatorname{Cov}\left[\eta_{t}, \xi_{t} \mid s_{t}=-1\right]=1.1061 \rho \sigma_{\eta}
\end{aligned}
$$

Inserting the values for $k$ and $v$ in 5.26 yields the linear state space model with trading volume and accounting for the leverage effect:

$$
\begin{aligned}
& \ln y_{t}^{2}=h_{t}+\lambda_{y} \ln V_{t}+\beta_{y}+\xi_{y, t}, \quad \xi_{y, t} \sim N\left(0, \pi^{2} / 2\right) \\
& h_{t+1}=\left(\varphi-0.2242 \rho \sigma_{\eta} s_{t}\right) h_{t}+s_{t} \sigma_{\eta} \rho\left(0.7979+0.2242 \ln y_{t}^{2}\right)+\eta_{t}^{+}
\end{aligned}
$$

Here $\eta_{i}^{+}$is distributed as:

$$
\eta_{t}^{*}-N\left(0, \sigma_{\eta_{p}}^{2}\left(1-0.8846 \rho^{2}\right)\right)
$$


The final model presented in 5.30 and 5.31 will be estimated by Quasi-Maximum Likelihood (QML), where $\sigma_{\xi}^{2}$ is set equal to $\pi^{2} / 2$. Before we estimate the model, we will first present the data set, perform several sign tests, Granger Causality tests and estimate linear VAR models as a motivation for our research into the volume-volatility relationship.

\subsection{Data Description and Summary Statistics}

The empirical analyses in this chapter are based on a sample of individual common stocks listed on the New York Stock Exchange (NYSE) $)^{10}$. This sample was used in a recent study by Lamoureux and Lastrapes (1994). The period under investigation starts January 3rd, 1967, and ends December 30th, 1987, spanning 5274 daily observations". The stocks we used in our empirical investigation are Alcoa, Amoco, Bristol-Myers, Champion and Hewlett-Packard respectively ${ }^{12}$. For each stock daily returns, measured as the logarithmic difference between two consecutive observations, and daily trading volumes were collected for the specified period. Trading volume is measured as the number of shares traded in a day ${ }^{13}$. Besides these five individual stocks we add daily returns of the S\&P 500 composite stock index and the corresponding daily volume of shares traded on the NYSE to our data set. The S\&P 500 is a value-weighted, arithmetic index of prices of common stocks. The S\&P series we obtained is identical to the data used in Gallant, Rossi and Tauchen (1992), though we restrict the sample size to the same period as the individual stock series. Daily volume is measured as the total number of shares that are traded in a day for all stocks that make up the S\&P 500. Returns and trading volumes of all series are adjusted for stock splits, stock-and cash dividends and capital modifications.

As Gallant, Rossi and Tauchen (1992), we are mainly interested in modelling the short-run patterns in conditional heteroskedasticity and volumes. Therefore we adjusted our data for systematic calendar effects, like weekends and holidays.

10 We are gratefull to Christopher Lamoureux and William Lastrapes for providing the data set. The total data set contains data for ten stocks. For reasons of space however, we decided to present results for five most actively traded stocks from this set. Results for the remaining stocks do not differ substantially from the results presented.

11 On Wednesdays in the last months of 1968, markets were closed due to paperwork backlog. We corrected for this phenomenon using non-trading day dummies.

12 These stocks will be abbreviated in the Tables as follows: ALC (Alcoa), AMO (Amoco), BRI (Bristol-Myers), CHA (Champion) and HEW (Hewlett-Packardy.

1. In Chapter 4 we saw that other measures for trading activity and intensity as for instance the daily number of transactions or the number of price quotes did not seem to make a big difference. We therefore decided to take trading volume measured as the numbers of shares traded on a given day. 
In order to adjust both price and volume data, we perform a two stage adjustment process in which systematic effects and deterministic trends are first removed from the mean and subsequently from the variance. In particular, we correct for day-of-the-week, non-trading day and month-of-the-year effects. In addition, we include filters for linear and quadratic trends. This procedure is similar to the adjustment procedure used in Gallant, Rossi and Tauchen (1992). We first regress logarithmic price differences and volume on the before mentioned set of adjustment variables. The resulting least squared residuals are taken from the mean equation to construct a variance equation. Then we standardize the residuals from the mean equation. This procedure results in adjusted price change and volume series with the same mean and variances as in the original sample. Figure 5.1 shows return and volume series of Hewlett Packard, for both original and adjusted series. Note that the adjustment procedure does not seem to affect the return series much. The unadjusted volume series exhibits a clear trend in level. This deterministic trend is successfully removed in the lower right panel of Figure 5.1.

Tables 5.1 through 5.3 provide summary statistics for the adjusted return series $\left(y_{1}\right)$, the adjusted $\log$ volume series $(\ln V)$ and a transformed return series ( $\ln$ $\left.y_{t}^{2}\right)$, respectively. The latter series are used as a proxy variable for volatility in the VAR and SV models that we will estimate in the next section. Table 5.1 shows that the adjusted return series feature slight negative skewness, considerable kurtosis and, measured by the Ljung-Box statistic, substantial serial correlation. Correcting for heteroskedasticity, by using the Diebold statistic (Diebold, 1988), reduces serial correlation considerably. All series are furthermore significantly first-order autocorrelated. Table 5.2 presents summaries for the adjusted log volume series. Clearly, the normality hypothesis can be rejected here too. In addition, this series is persistently autocorrelated and serially correlated. The transformed return series in Table 5.3 are substantially negatively skewed, implied by the log transformation and related to the so called "inlier" problem" The variance of each transformed series is higher than that of a log-chi-squared (equal to $\pi^{2} / 2$ ). Other features are again the significant serial correlation and persistent autocorrelation. For all three series the hypothesis of a unit root, as indicated by the augmented Dickey-Fuller test statistic, can be clearly rejected.

14 See for instance Mahieu and Schotman (1996). 
Table 5.1 Summary Statistics Adjusted Stock Retums

\begin{tabular}{|c|c|c|c|c|c|c|}
\hline & ALC & AMO & BRI & $\mathrm{CHA}$ & HEW & S\&P 500 \\
\hline Mean & 0.052 & 0.064 & 0.060 & 0.046 & 0.078 & 0.024 \\
\hline St. dev. & 1.754 & 1.588 & 1639 & 2.023 & 2,074 & 1.140 \\
\hline Skewness & -0.535 & -0.578 & .0396 & 0.008 & -0.716 & -0.513 \\
\hline Kurtosis & 17.398 & 20.459 & 28.380 & 6.596 & 17.305 & 17.043 \\
\hline Minmum & -25.386 & -26.564 & -27.806 & -18.403 & -31.677 & -18.025 \\
\hline Maximum & 14.422 & 8.494 & 20.325 & 12.011 & 10.883 & 7.696 \\
\hline $\operatorname{ADF}(4)$ & $-37.041^{\circ}$ & $-34118^{*}$ & $-33225^{\prime}$ & -32694 & $-32.353^{\prime \prime}$ & $-31.593^{\prime \prime}$ \\
\hline LB (25) & $195.5^{\circ}$ & $104.7^{*}$ & $125.1^{\circ}$ & $65.9^{\prime \prime}$ & $57.3^{x}$ & $2234^{\circ}$ \\
\hline LB (50) & $254.4^{*}$ & $152.1^{\circ}$ & $152.0^{*}$ & $95.9^{\prime \prime}$ & $99.4^{\circ}$ & $274.6^{\circ}$ \\
\hline$D(25)$ & 43.1 & 42.0 & 31.8 & $47.3^{\prime \prime}$ & 27.6 & $729^{\prime \prime}$ \\
\hline$D(50)$ & $79.4^{\circ}$ & 75.3 & 50.6 & 72.7 & 56.1 & $107.7^{\circ}$ \\
\hline $\mathrm{AC}$ (1) & $0.163^{\prime \prime}$ & $0.110^{\circ}$ & $0.070^{\circ}$ & $0.074^{*}$ & $0.059^{\circ}$ & $0.193^{\circ}$ \\
\hline$A C(2)$ & -0.018 & -0.034 & -0.059 & 0.005 & -0.025 & -0.014 \\
\hline$A C(3)$ & -0.042 & -0.026 & -0.058 & -0.006 & -0.013 & 0.003 \\
\hline$A C(4)$ & -0.029 & -0.033 & -0.040 & -0.017 & -0.010 & -0.010 \\
\hline$A C(5)$ & -0.016 & -0.026 & 0.044 & -0.009 & 0.014 & 0.002 \\
\hline $\mathrm{AC}(10)$ & -0.013 & -0.002 & -0.052 & -0.005 & -0.001 & -0.017 \\
\hline$A C(20)$ & -0.012 & -0.021 & 0.010 & 0.002 & -0.026 & -0.010 \\
\hline
\end{tabular}

Table 5.2 Summary Statistics Adjusted Log Trading Volumes

\begin{tabular}{|c|c|c|c|c|c|c|}
\hline & $A L C$ & AMO & $\mathrm{BR}$ & $\mathrm{CHA}$ & HEW & $58 P 500$ \\
\hline Mean & 6.111 & 6.570 & 6.250 & 5.958 & 6.119 & 8.347 \\
\hline St. dev. & 1.381 & 1.386 & 1.347 & 1.503 & 1.744 & 0.478 \\
\hline Skewness & -0.183 & -0.328 & -0.091 & -0.161 & -0.271 & 0.233 \\
\hline Kurtosis & 4.529 & 6.914 & 6.700 & 4.677 & 6.915 & 2.741 \\
\hline Minimum & -3.760 & -5.296 & -3.691 & -3.565 & -11.227 & -6.173 \\
\hline Maximum & 13.244 & 14.189 & 15.229 & 12.852 & 15.962 & 10.153 \\
\hline $\operatorname{ADF}(4)$ & $-20.560^{\circ}$ & $-16.033^{*}$ & $-17.721^{\circ}$ & $-19.211^{*}$ & $-18.890^{\circ}$ & $-10.106^{\circ}$ \\
\hline LB (25) & $5364.7^{*}$ & $7645.3^{\circ}$ & $9627.3^{\circ}$ & $6342.9^{\circ}$ & $8865.0^{\circ}$ & $530193^{2}$ \\
\hline LB (50) & $7864.2^{*}$ & $9534.7^{*}$ & $12701.5^{*}$ & $7844.3^{*}$ & $12622.0^{\circ}$ & $87710.8^{\prime \prime}$ \\
\hline$D(25)$ & $3988.6^{\circ}$ & $4623.4^{\circ}$ & $4986.6^{\circ}$ & $4303.8^{*}$ & $6010.5^{\circ}$ & $34428.3^{\prime}$ \\
\hline$D(50)$ & $4645.5^{\circ}$ & $6778.7^{\circ}$ & $7127.3^{\circ}$ & $5592.6^{*}$ & $8951.4^{*}$ & $60729.3^{\circ}$ \\
\hline$A C(1)$ & $0.383^{\circ}$ & $0.544^{\circ}$ & $0.452^{\prime \prime}$ & $0.371^{\prime \prime}$ & $0.447^{\circ}$ & $0.837^{\circ}$ \\
\hline$A C(2)$ & $0.309^{*}$ & $0.456^{\circ}$ & $0.364^{\prime \prime}$ & $0.322^{*}$ & $0.379^{\circ}$ & $0.759^{\circ}$ \\
\hline $\mathrm{AC}(3)$ & $0.260^{\circ}$ & $0.416^{\circ}$ & $0.342^{\prime \prime}$ & $0.294^{*}$ & $0.328^{\circ}$ & $0.734^{\circ}$ \\
\hline$A C(4)$ & $0.249^{\circ}$ & $0.420^{\circ}$ & $0.325^{\prime \prime}$ & $0.264^{4}$ & $0.312^{\circ}$ & $0.717^{\prime}$ \\
\hline$A C(5)$ & $0.217^{\prime \prime}$ & $0.390^{\circ}$ & $0.317^{4}$ & $0.260^{\circ}$ & $0.275^{\circ}$ & $0.703^{\circ}$ \\
\hline$A C(10)$ & $0.184^{\prime \prime}$ & $0.332^{\circ}$ & $0.273^{\prime \prime}$ & $0.250^{\circ}$ & $0.264^{*}$ & $0.640^{\circ}$ \\
\hline$A C(20)$ & $0.136^{\circ}$ & $0.313^{\circ}$ & $0.217^{\circ}$ & $0.155^{*}$ & $0.226^{*}$ & $0.577^{\circ}$ \\
\hline
\end{tabular}

Notes for tables 5.1 and 5.2: Sample period Januany 1967 -December 1987:5.274 observations. ALC = Alcoa; $\mathrm{AMO}=\mathrm{Amoca} ; \mathrm{BRI}=\mathrm{Bristol}-\mathrm{Myers} ; \mathrm{CHA}=$ Champion; HEW = Hewlett-Packard; S\&P $500=$ Standard and Poors Composite Stock Index. The adjustment procedure is explained in the text. ADF(4) denotes the augmented Dickey-Fuller test with 4 lags. The $1 \%$ Mackinnon critical value for rejection of the hypothesis of a unit root is -3.43 . LB $(p)$ denotes the Ljung-Box statistic for serial correlation using $p$ autocorrelations; $D(p)$ is the Diebold (1988) heteroskedasticity corrected serial correlation test, and AC(p) denotes the sample autocorrelation at lag $p$. "denotes statistical significance at the $1 \%$ level, using heteroskedasticity consistent standard errors. 

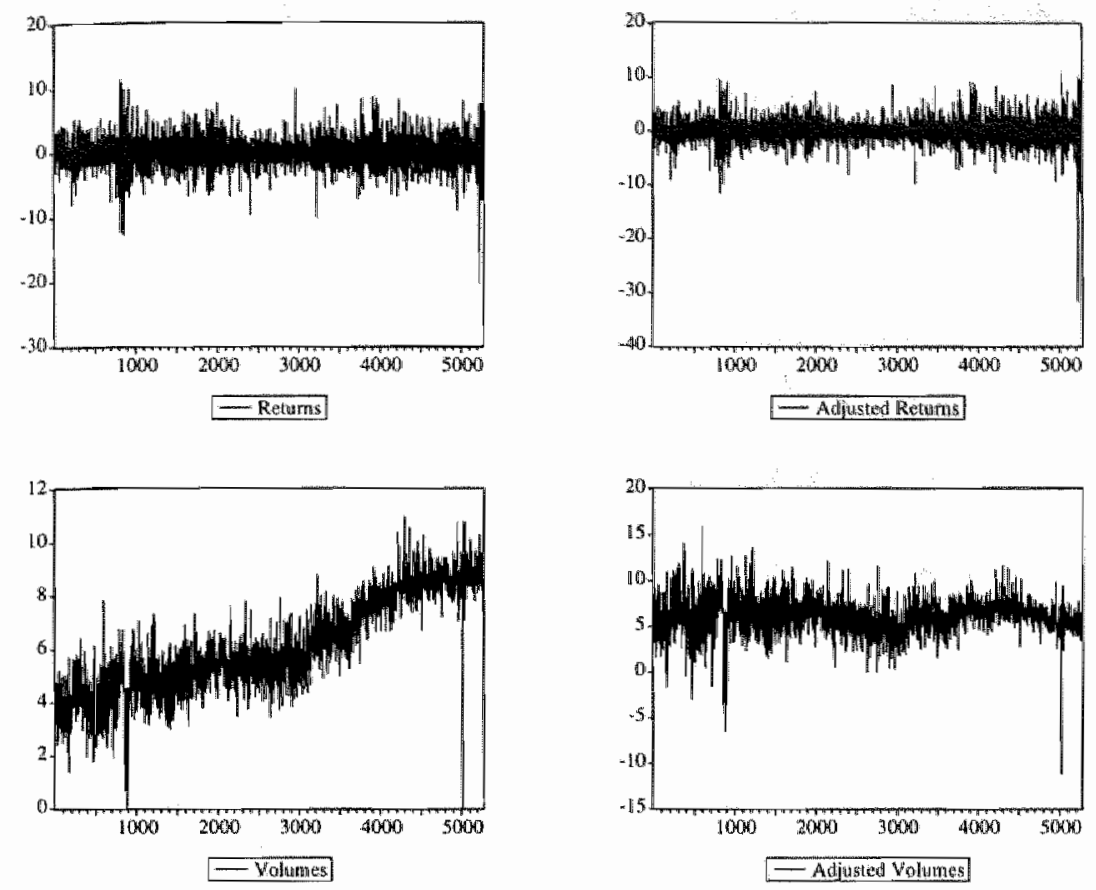

Figure 5.1 Unadjusted and Adjusted Returns (\%) and Volumes for Hewlett Packard 1967-1987.

\subsection{Sign Bias Tests}

In the empirical literature on stock price behaviour some attention is given to the observation that positive and negative innovations to the return process have different impacts on the conditional volatility. This so-called leverage effect was initially described by Black (1976), who found that negative shocks had a much bigger impact on conditional volatility than positive shocks. The explanation Black gives is that a negative shock to a firm's stock price increases its leverage and thereby the expected variance of its stock. Glosten, Jagannathan and Runkle (1993) argue that the leverage effect is related to expected future cash flows. Assume that discount rates are constant. If an unexpected negative shock in expected future cash flows occurs, which decreases the stock price, and the variance of future expected cash flows does not change proportionally, the variance of future expected cash flows per dollar of the stock price will rise. Consequently, future returns will be more volatile. 
Table 5.3 Sunmary Statistics log Squared Adjusted Returns

\begin{tabular}{|c|c|c|c|c|c|c|}
\hline & ALC & AMO & BAI & $\mathrm{CHA}$ & HEW & $58 \mathrm{P} 500$ \\
\hline Meăin & 9919 & -10.139 & -9.947 & -9496 & -9.543 & -10.574 \\
\hline St. dev. & 2.474 & 2.529 & 2.356 & 2.431 & 2511 & 2361 \\
\hline Skewness & -1.082 & -1.248 & -1.125 & -1.133 & -1.358 & -1.265 \\
\hline Kurtosis & 4.828 & 6.117 & 5.059 & 5309 & 6.748 & 6.048 \\
\hline Minimum & -23.926 & -26.629 & -22.748 & -25.894 & -28.070 & $\cdot 27.453$ \\
\hline Maximum & -2.742 & -2.659 & -2.560 & -3385 & -2.299 & -3.427 \\
\hline $\operatorname{ADF}(4)$ & $-26.052^{\prime \prime}$ & $-23.991^{*}$ & $-27.101^{\circ}$ & $-26.944^{\circ}$ & $-26.113^{\circ}$ & -26.806 \\
\hline$\angle B(25)$ & $620.0^{*}$ & $1570.7^{\prime \prime}$ & $455.9^{\prime \prime}$ & $420.0^{\circ}$ & $767.6^{\circ}$ & $637.8^{\circ}$ \\
\hline$\angle B(50)$ & $9138^{\prime \prime}$ & $2532.3^{\prime \prime}$ & $696.2^{*}$ & $533.2^{\prime \prime}$ & $1146.2^{*}$ & $1025.7^{\circ}$ \\
\hline $\mathbb{D}(25)$ & $588.5^{\circ}$ & $1483.6^{*}$ & $455.3^{\prime \prime}$ & $375.2^{*}$ & $760.2^{\circ}$ & $637.3^{*}$ \\
\hline$D(50)$ & $878.8^{\prime \prime}$ & $2437.6^{\circ}$ & $681.3^{\circ}$ & $49 \| 3^{\prime \prime}$ & $1136.4^{\circ}$ & $1013.9^{\circ}$ \\
\hline$A C(1)$ & $0.107^{\circ}$ & $0.167^{\circ}$ & $0.127^{*}$ & $0.105^{\circ}$ & $0.139^{\circ}$ & $0.078^{*}$ \\
\hline$A C(2)$ & $0.087^{*}$ & $0.151^{*}$ & $0.102^{*}$ & $0.078^{*}$ & $0.106^{\circ}$ & $0.057^{\prime \prime}$ \\
\hline$A C(3)$ & $0.076^{\prime \prime}$ & $0.121^{\circ}$ & $0.066^{\circ}$ & $0.072^{\circ}$ & $0.075^{\circ}$ & $0.043^{*}$ \\
\hline$A C(4)$ & $0.084^{\prime \prime}$ & $0.102^{\circ}$ & $0.050^{\circ}$ & $0.083^{\prime \prime}$ & $0.079^{\circ}$ & $0.066^{\circ}$ \\
\hline$A C(5)$ & $0.089^{\prime \prime}$ & $0.139^{\circ}$ & $0.075^{\circ}$ & $0.061^{\circ}$ & $0.089^{\prime \prime}$ & $0.093^{\circ}$ \\
\hline$A C(10)$ & $0.063^{\circ}$ & $0.108^{\circ}$ & $0.055^{\circ}$ & $0.061^{\prime \prime}$ & $0.087^{\circ}$ & $0.051^{\circ}$ \\
\hline$A C(20)$ & 0.040 & $0.100^{*}$ & $0.051^{*}$ & $0.049^{\prime \prime}$ & $0.069^{\circ}$ & 0.066 \\
\hline
\end{tabular}

Sample period lanuary 1967 - December 1987; 5274 observations. $A L C=$ Alcoa; $A M O=A m o c o ; B R I=$ Bristol-Myers; CHA $=$ Champion; HEW = Hewlett-Packard; $5 \&$ P $500=$ Standard and Poors Composite Stock Index. The adjustment procedure is explained in the text. ADF(4) denotes, the augmented Dickey-Fuller test with 4 lags. The $1 \%$ Mackirnon critical value for rejection of the hypothesis of a unit root is -3.43 . LB(p) denotes the Ljung-Box statistic for serial correlation using $p$ autocorrelations: $D(p)$ is the Diebold (1988) heteroskedasticity corrected serial correlation test, and $A C(p)$ denotes the sample autocorrelation at lag $p$. "denotes statistical significance at the $1 \%$ level, using heteroskedasticity consistent standard errors.

Campbell and Hentschel (1992) develop a model in which they explicitly stress the link from conditional volatility to expected returns. Large pieces of news (either good or bad) on future dividends lead to falls in stock prices because they increase the required rate of return on stock prices. The drop in stock prices is damped in case of good news (higher future dividends) or amplified in case of bad news (lower future dividends). In contrast, small news events lead to lower volatility, lower required rates of return and higher stock prices. Campbell and Hentschel (1992) call the link between (conditional) volatility and required rates of return volatility feedback. Although the causality between stock prices and volatility differs among the studies in this paragraph, they all stress the importance of correlation between volatility and stock returns. Engle and $\mathrm{Ng}$ (1993) compare several models of the ARCH class with respect to their abilities to capture the leverage effect. The main tool they used is the news impact curve (NIC) that plots the conditional volatility $\left(h_{i}\right)$ against the innovation $\left(\varepsilon_{t-1}\right)$ in the return series $y_{t}$. They also introduced some diagnostic tests based on the signs of a time series. The tests can be performed both on the residuals of a volatility model as well as on the original data $y_{t}$. In the latter case let us define $\varepsilon_{t} \equiv y_{p}-m_{y}$ and $v_{t} \equiv \varepsilon_{t} / s_{y}$, where $m_{y}$ and $s_{y}$ are the unconditional mean and standard deviation of $y_{k}$, respectively. Also define: 
$\delta\left(\varepsilon_{t-1}\right)= \begin{cases}1 & \text { if } \varepsilon_{t-1}<0 \\ 0 & \text { otherwise, }\end{cases}$

and $\delta^{+}\left(\varepsilon_{i-1}\right)$ analogously. The Sign Bias Test (SBT) is given by the t-statistic on $\alpha_{1}$ of the following least squares regression:

$\varepsilon_{t}^{2}=\alpha_{0}+\alpha_{1} \delta^{-}\left(\varepsilon_{t-1}\right)+u_{t}$

This test examines the impact of negative and positive shocks to the squared innovations. The Negative Sign Bias Test (NSBT) and Positive Sign Bias Test (PSBT) test whether the size of the innovations matters conditional on the innovations being negative or positive, respectively. Note that it does not matter if we have either $\varepsilon_{t}^{2}$ or $v_{t}^{2}$, because the constant scaling factor in the definition does not influence the regression results. Here, the relevant regressions are:

NSBT: $\quad \varepsilon_{t}^{2}=\alpha_{0}+\alpha_{1} \delta\left(\varepsilon_{t-1}\right) \varepsilon_{t-1}+u_{t}$

PSBT: $\quad \varepsilon_{t}^{2}=\alpha_{0}+\alpha_{1} \delta^{+}\left(\varepsilon_{t-1}\right) \varepsilon_{t-1}+u_{t}$

Engle and $\mathrm{Ng}$ (1993) also report a Joint Sign Bias Test (JSBT) in which all three individual tests are combined. It is an LM test for adding three extra variables under a model for the conditional volatility. In case of a diagnostic test for the raw data this test is equal to a standard $F$-test. The regression for the JSBT is:

$$
\varepsilon_{t}^{2}=\alpha_{0}+\alpha_{11} \delta^{-}\left(\varepsilon_{t-1}\right)+\alpha_{12} \delta^{-}\left(\varepsilon_{t-1}\right) \varepsilon_{t-1}+\alpha_{13} \delta^{+}\left(\varepsilon_{t-1}\right) \varepsilon_{t-1}+u_{t}
$$

The test statistic is then given by $T$ times the $R$-squared from this regression and is asymptotically chi-squared distributed with 3 degrees of freedom. Results are reported in Table 5.4 for the raw data. We use the residuals from an autoregression of order 15 in order to dellete all possible serial dependence in $y_{1}$. There appears to be no strong evidence for an asymmetric effect of innovations to volatility as is shown by the insignificant coefficients $\alpha_{1}$ in the SBT. The NSBT and PSBT results show that a size effect seems to be present: large negative/positive shocks have more effect on volatility than smaller shocks. These results remain qualitatively the same when we include the logarithm of lagged volume ( $\left.\ln V_{\mathrm{b}-1}\right)$ as an extra regressor in the autoregression. Although the leverage effect is considered to be an intrinsic characteristic of stock prices, Gallant, Rossi and Tauchen (1992) have shown that the leverage effect arises predominantly as a tail phenomenon in the distribution of the S\&P 500 index series from 1928 to 1987 . When large negative (and positive) shocks are deleted from the sample, the evidence for existence of the leverage effect vanishes quickly. This can be tentatively shown by doing the following regression: 
Table 5.4 Sign Bias Tests

\begin{tabular}{|c|c|c|c|c|c|c|c|}
\hline & & $\mathrm{ALC}$ & AMO & $\mathrm{BRI}$ & $\mathrm{CHA}$ & HEW & S\&P \\
\hline \multirow[t]{2}{*}{ SBT } & $a_{1}$ & 0.164 & 0.258 & 0.153 & 0.015 & 0.273 & 0.162 \\
\hline & $t$ & 0.494 & 0.862 & 0.405 & 0.056 & 0.573 & 1.215 \\
\hline \multirow[t]{2}{*}{ NSBT } & $a_{i}$ & -1.994 & -1.725 & -2.875 & -0.654 & -1.556 & -1.265 \\
\hline & $t$ & -12.251 & -11.036 & -15.623 & -6.026 & -8.485 & -13.071 \\
\hline \multirow[t]{2}{*}{ PSBT } & $\alpha_{1}$ & 0.665 & 0.474 & 1.752 & 0.514 & 0.759 & 0.263 \\
\hline & $t$ & 2.249 & 3.137 & 9.312 & 4.885 & 3.985 & 2.668 \\
\hline ISET & $\mathbb{M}$ & 231.9 & 177.0 & 465.6 & 92.9 & 123.8 & 250.5 \\
\hline \multicolumn{8}{|c|}{ Volume; Corrected Series } \\
\hline \multirow[t]{2}{*}{ SBT } & $\alpha_{i}$ & 0.188 & 0.351 & 0.189 & 0.163 & -0.314 & 0.154 \\
\hline & $t$ & 0.562 & 1.163 & 0.499 & 0.619 & 0.658 & 1.117 \\
\hline \multirow[t]{2}{*}{ NSBT } & $a_{i}$ & -1.932 & -1.722 & -2.858 & -0.667 & -1.561 & -1.266 \\
\hline & $t$ & -12.514 & -11.093 & -15.540 & -6.194 & -8.531 & -12.752 \\
\hline \multirow[t]{2}{*}{ PSBT } & $\alpha_{1}$ & 0.656 & 0.445 & 1.755 & 0.468 & 0.752 & 0.225 \\
\hline & $t$ & 4.120 & 2.889 & 9.261 & 4.358 & 3.931 & 2.164 \\
\hline ISBT & $L M$ & 237.7 & 173.8 & 459.7 & 87.7 & 123.8 & 232.0 \\
\hline
\end{tabular}

Results are based on residuals of an AR(15) (upper part) and an AR(15) plus lagged trading volume (lower parth. SBT denotes the Sign Bias Test, N5BT the Negative Sign Bias Test, PSBT the Positive Sign Bias Test and JSBT the Joint Sign Bias Test. See text for a detailed description of the regression equations. Reported are the coefficients on the sign variables together with the t-statistic (SBT, NSBT, PSBT) and the LM test statistic $\left(X^{2}(3)\right.$ distributed) for JSBT. See Table 5.1 for a description of the series.

$$
\left|y_{t}\right|=\alpha_{0}+\alpha_{1}\left|y_{t-1}\right|+\beta\left(y_{t-1} \delta\left(y_{t-1}\right)\right)+u_{t}
$$

Here we use the absolute value of the return series for describing volatility. Thus, an autoregressive process of order 1 is fitted to the data in which negative returns are treated differently than positive returns through the term $y_{t-1} \delta\left(y_{t-1}\right)$. The coefficient $\beta$ captures the leverage effect. The null hypothesis of no leverage is that $\beta$ is equal to zero. In the upper part of Table 5.4 we present results from doing the regressions in 5.37 for the five stock return series and the S\&P 500 index. The cut-off points in the first column are determined from the unconditional standard deviations $s_{y}$ of the $y_{1}$, series. Thus, at a particular $c$, we restrict our sample to those points $\left(y_{t}, y_{t-1}\right)$ that obey the following restrictions:

$$
\left|y_{t}\right|<s_{y} c \text { and }\left|y_{t-1}\right|<s_{y} c
$$

When $c=\infty$, the whole sample $y_{1}(t=1, \ldots, T)$ is used. From the table we see that the coefficients $\beta$ are generally insignificant for lower cutoff points. Furthermore, a significant leverage effect can only be found in three series (Alcoa, Amoco and the S\&P 500). In case of Alcoa only two observations (related to the October 1987 crash) cause $\beta$ to become significantly negative. Results for the S\&P 500 index series suggest that this series seems to have a significant leverage 
effect. In addition, Gallant, Rossi and Tauchen (1992) provide evidence for the disappearance of the leverage effect once trading volume is taken into account. They perform a similar regression as 5.37 in which the asymmetry coefficient is now a linear function of trading volume:

$$
\left|y_{t}\right|=\alpha_{0}+\alpha_{1}\left|y_{t-1}\right|+\left(\gamma_{0}+\gamma_{1} \ln V_{t-1}\right) y_{t-1} \delta^{-}\left(y_{t-1}\right)+u_{t}
$$

The lower part of Table 5.5 shows the results from this regression. The regression coefficients:

$\beta=\hat{\gamma}_{0}+\hat{\gamma}_{1} \ln V_{t-1}$

are evaluated at the volumes:

$$
\ln V_{t-1}=m_{V}+k s_{V}, \quad(k=-2,-1,0,1,2),
$$

In conclusion, the exercises above show that evidence for a leverage effect is rather mixed. Engle and Lee (1993) provide evidence that there is no leverage effect in the long run in a model where conditional volatility is decomposed into a permanent and a transitory component. In our data series it seems that there is a sigmificant leverage effect for the S\&P 500 index, however not for individual stock series. Furthermore, the interactions with volume are not clear from the regression results. LeBaron (1993) indicates that a parametric model that jointly describes stock returns and trading volume, needs to possess some nonlinear features. Nevertheless, in some of the models we estimate below we will allow the possibility of a link between the conditional variance and innovations to the return process. It remains an open question, though, to what extent results from this link can be interpreted as a leverage effect. 
Table 5.5 Leverage Regressions

\begin{tabular}{|c|c|c|c|c|c|c|c|}
\hline$c$ & & ALC & AMO & $\mathrm{BRH}$ & $\mathrm{CHA}$ & HEW & S\&P 500 \\
\hline \multirow[t]{3}{*}{1} & $B$ & 0.015 & 0.014 & 0.025 & 0.019 & $=0.007$ & -0.007 \\
\hline & $s(\beta)$ & 0.019 & 0.021 & 0019 & 0.019 & 0.020 & 0.019 \\
\hline & $T$ & 4888 & 4821 & 4897 & 4837 & 4843 & 4850 \\
\hline \multirow[t]{3}{*}{2} & $\beta$ & 0.004 & -0.048 & 0.022 & -0.017 & -0.004 & -0.068 \\
\hline & $s(B)$ & 0.020 & 0.020 & 0.018 & 0.019 & 0.019 & 0.018 \\
\hline & $T$ & 5229 & 5229 & 5233 & 5232 & 5228 & 5222 \\
\hline \multirow[t]{3}{*}{3} & $B$ & 0.024 & -0.049 & 0.032 & 0.000 & 0.021 & -0.067 \\
\hline & $s(\beta)$ & 0.020 & 0.020 & 0.018 & 0.019 & 0.019 & 0.018 \\
\hline & $T$ & 5263 & 5265 & 5260 & 5267 & 5269 & 5266 \\
\hline \multirow[t]{3}{*}{4} & $B$ & 0.036 & -0.057 & 0.002 & -0.001 & 0.000 & -0.041 \\
\hline & $s(\beta)$ & 0.020 & 0.020 & 0.020 & 0.019 & 0.019 & 0.018 \\
\hline & $T$ & 5269 & 5271 & 5269 & 5273 & 5270 & 5272 \\
\hline \multirow[t]{3}{*}{5} & $B$ & 0.021 & -0.049 & -0.006 & -0.001 & 0.004 & -0.064 \\
\hline & $s(\beta)$ & 0.020 & 0.019 & 0.019 & 0.019 & 0.019 & 0.019 \\
\hline & $T$ & 5271 & 5273 & 5270 & 5273 & 5273 & 5273 \\
\hline \multirow[t]{3}{*}{$\infty$} & $\beta$ & -0.042 & -0.049 & 0.003 & -0.001 & 0.004 & -0.064 \\
\hline & $5(\beta)$ & 0.019 & 0.019 & 0.019 & 0.019 & 0.019 & 0.019 \\
\hline & $T$ & 5273 & 5273 & 5273 & 5273 & 5273 & 5273 \\
\hline $\mathrm{k}$ & & ALC & AMO & BAI & CHA & HEW & S\&P 500 \\
\hline \multirow[t]{2}{*}{-2} & $\beta$ & 0.086 & 0.069 & 0.080 & -0.070 & -0.014 & -0.019 \\
\hline & $s(\beta)$ & 0.036 & 0.042 & 0.036 & 0.035 & 0.040 & 0.029 \\
\hline \multirow[t]{2}{*}{-1} & $\beta$ & 0.031 & 0.023 & 0.050 & -0.039 & -0.007 & -0.038 \\
\hline & $s(\beta)$ & 0.026 & 0.030 & 0.026 & 0.025 & 0.029 & 0.023 \\
\hline \multirow[t]{2}{*}{0} & $\beta$ & -0.026 & -0.023 & 0.020 & -0.008 & 0.000 & -0.058 \\
\hline & $s(\beta)$ & 0.020 & 0.021 & 0.020 & 0.020 & 0.021 & 0.019 \\
\hline \multirow[t]{2}{*}{1} & $\beta$ & -0.081 & -0.068 & -0.010 & 0.022 & 0.007 & -0.077 \\
\hline & $s(\beta)$ & 0.021 & 0.020 & 0.020 & 0.021 & 0.020 & 0.020 \\
\hline \multirow[t]{2}{*}{2} & $\beta$ & -0.137 & -0.114 & -0.040 & 0.052 & 0.014 & -0.097 \\
\hline & $s(\beta)$ & 0.029 & 0.028 & 0.026 & 0.030 & 0.027 & 0.025 \\
\hline
\end{tabular}

The upper part of this table shows the asymmetry coefficient and its standard error in the least squares regression in equation 5.37, which is truncated to points $\left(y_{t}, y_{i, j}\right)$ that have both elements to be smaller than $c s_{y}$ in absolute walue. $T$ is the number of observations used in the regression. The lower part of the table presents the asymmetry coefficients $\beta$, (see equation 5.40 ), from the regression in equation 5.39 evaluated at volumes $m_{y}+k s_{y,}$ with $m_{y}$ and $s_{y}$ the unconditional mean and standard deviation of $I n v_{p, t}$. See Table 5.1 for the description of the series.

\subsection{VAR Models and Granger Causality Tests}

A useful way to get a preliminary insight in the joint dynamics of a system of time series is to estimate Vector AutoRegressive (VAR) models. These models were first introduced by Sims (1980). A VAR(p) model can be written as:

$x_{t}=\gamma+\sum_{i=\mathcal{I}}^{p} A_{i} x_{t-i}+u_{t r} \quad t=1, \ldots, T$.

The vector $x_{i}=\left(x_{1 t}, \ldots, x_{k}\right)^{\prime}$ is a $(K \times 1)$ vector that stacks $K$ time series. The $(K \times 1)$ vector $\gamma$ is a vector with intercepts and the $(K \times K)$ matrices $A_{i}(i=1, \ldots, p)$ contain 
further coefficients. The $K^{\prime}$-dimensional innovation process $u_{t}=\left(u_{\eta}, \ldots, u K_{t}\right)^{\prime \prime}$ has the properties $\mathrm{E}\left(u_{i}\right)=0, \mathrm{E}\left(u_{i} u_{i}^{\prime}\right)=\Sigma$, and $\mathrm{E}\left(u_{i} u_{p}^{\prime}\right)=0$ for $t \neq s$. Estimation of the VAR processes can be done by performing Least Squares on each of the $K$ time series, see Lütkepohl (1991). The data series we use in our estimation procedure are the percentage return $\left(y_{t}\right)$, the daily logarithmic volume $\left(\ln V_{i}\right)$ and a proxy variable for volatility $\left(\ln y_{t}^{2}\right)$. The volatility series is motivated by the log-square transformation of returns that is used for estimating our stochastic volatility models in the next section ${ }^{15}$.

In a VAR analysis we can investigate the linear dynamic interactions between the variables above. Note that our results can be indicative only due to the exact non-linear relationship between returns and volatility. Our focus will be on the relation that trading volume has on the return and volatility process. Interesting questions that can be raised in this context are related to, among others, the lead/lag relations of volume, and the impact of shocks in volume on stock returns and volatility. As we will show in the remainder of this chapter, there is strong evidence for heteroskedasticity in the volatility series. This could lead to inconsistent estimation of the variance-covariance matrix of the parameters, see Hamilton (1994). This matrix is particularly important for the analysis in this section. We performed a Newey-West procedure in order to correct the covariance matrix of the parameters. We experimented with the appropriate lag length, but results did not change substantially. The results below are based on a lag length of 30 in the Newey-West procedure.

A first important step in every VAR analysis is the determination of the order $p$. Lütkepohl (1991) discusses several ways how this can be done properly. We decided to use two methods: a likelihood-based criterion and the Akaike Information Criterion (AIC). The likelihood method compares the log-likelihood values in a sequential testing procedure. Suppose we know the maximum order $M$ of the VAR. Subsequently we estimate the VAR for $p=M, \ldots, 1$ and compute the following test statistic:

$$
\lambda(p)=T\left(\ln \left|\tilde{\Sigma}_{H}(M-p)\right|-\ln \left|\Sigma_{H}^{\infty}(M-p+1)\right|\right) .
$$

where $\Sigma_{H}(p)$ is the heteroskedastic-consistent maximum likelihood estimator of $\Sigma$ for the VAR(p) model. The null hypothesis for this test is that the matrix $A_{p}$ is zero conditional on $A_{\mathrm{p}+1}, \ldots, A_{M}$ being zero. The test statistic has an asymptotic $\chi^{2}\left(K^{2}\right)$ distribution. For our series we set $M=20$ and we test at a $1 \%$ significance 
level ${ }^{16}$. The AIC method for determining the order of a VAR model is computed as follows, see Lütkepohl (1991):

$$
A I C(p)=\ln \left|\tilde{\Sigma}_{H}(p)\right|+\frac{2 p K^{2}}{T}
$$

The optimal order for $p$ is found by minimizing AIC( $p)$. In Table 5.6 we present the optimal orders for the trivariate VAR models, with $x_{t}=\left(y_{1}, \ln y_{t}{ }^{2}, \ln V_{1}\right)^{2}$. Remarkably, all orders are around 10 , which corresponds with 10 trading days ${ }^{17}$. Our findings are in fact consistent with results in LeBaron (1993) and Hiemstra and Jones (1994), who also perform VAR analyses on systems that include daily trading volume. In the analyses below we chose the largest order for each VAR system.

The optimal orders found for all VAR models prohibit the presentation of the estimated coefficients. Furthermore ${ }_{i}$ in higher order $(p>1)$ VARs the dynamic interactions among the variables cannot be readily inferred by simply looking at the parameters. A more useful technique for analysing the dynamics is to compute impulse response functions (IRFs).

Table 5.6 Optimal VAR Orders

\begin{tabular}{lllllll}
\hline & ALC & AMO & BRI & CHA & HEW & S\&P 500 \\
\hline Likelihood & 10 & 13 & 11 & 11 & 11 & 12 \\
AIC & 9 & 12 & 10 & 10 & 10 & 11 \\
\hline
\end{tabular}

All entries are orders $p$ for the VAR(p) model. Likelihood refers to the likelihood based test procedure described in the text. The significance level is $1 \%$. A/C is the Aikake information Criterion also described in the text.

An IRF traces the response of the endogenous variables in the system to shocks in the errors. In particular, it separates the determinants of the endogenous variables into shocks or innovations identified with specific variables in the system. It then traces the effect on current and future values of the endogenous variables of one standard deviation shocks to the innovations. If the errors in the system equations are uncorrelated the interpretation of the IRFs is straightforward. However, it is almost never the case that these errors are uncorrelated as they have a common component which cannot be identified. An arbitrary, but often used, method of dealing with this problem is to attribute all of the effect of

\footnotetext{
16 We repeated this procedure for a $5 \%$ significance level, but the results were identical to the ones reported.

17 This could be caused by a remaining seasonality (a two-week effect?) in the series.
} 
any common component to the variable that comes first in the VAR model ${ }^{18}$. Changing the order of the equations in the VAR can dramatically change the IRFs and therefore care should be given to interpreting the impulse response functions.

We present IRFs for the trivariate VAR systems for the following order of variables: volume, returns and volatility (see Appendix A). The order we have selected is chosen arbitrarily. We computed the IRFs for all remaining order combinations, but there was no significant influence on results and therefore we decided to only give results for one order. In Figure 5.2 we present the IRFs for volatility and volume, implied by the trivariate VAR and the accompanying VAR orders. For all stocks a shock in trading volume has a substantial, positive effect on cumulative volatility. If we look closer however we see for Champion, Bristol Myers and the S\&P 500 a one time increase in volatility, but in the following days this effects slowly fades away or remains constant ${ }^{19}$. The effect of a shock in volatility to trading volume is less strong, although present (both negatively or positively) for all stocks.

In Figure 5.3 we present IRFs for returns and volume. A shock in trading volume also has an impact on the return level, but it varies across stocks. A response of volume to a shock in returns gives the same mixed results: for Alcoa, Amoco and the S\&P 500 series a shock in the return level leads to a higher cumulative volume level, whereas the opposite is the case for Bristol Myers and Hewlett Packard. These results more or less confirm our VAR estimations. It is however interesting to find a difference between the reaction on a individual level (stocks) and an aggregate level (index).

Another way of investigating the dynamic properties of a VAR system is to test for Granger Causality, see Hamilton (1994). A variable $y$ is said to be Granger caused by $z$ if $z$ helps in the predictions of $y$, or equivalently if the coefficients on the lagged $z$ 's are statistically significant. In a VAR system a test for Grangercausality amounts to a joint test for zero coefficients in the $A_{i}(i=1, \ldots, p)$ matrices. For example, if we want to test if the second variable in a VAR Granger causes the first variable, the null hypothesis is that elements $(1,2)$ in every $A_{i}$ are equal to zero. The test statistic turns out to be a standard $F$-test with degrees of free-

18 The errors are orthogonalized by a Choleski decomposition so that the covariance matrix of the innovations is lower triangular.

19 As mentioned before the ordering of the variables in the VAR model is of importance and therefore we investigated the effect of a different ordering schedule. We made the IRFs for all combinations (6) of volume, volatility and returns. For some series we see more or less pronounced positive or negative responses, but generally the direction of the response remains the same. 
dom equal to $p$ and $T-p K-1$. Again we took account of possible heteroskedasticity in the data when we compute the test statistics. In Tables 5.7 and 5.8 we report results for Granger-Causality tests for the bivariate relationship between tracling volume and stock returns on the one hand and trading volume and stock volatility on the other hand. We use several lag structures in order to test for consistency of the test statistics. Table 5.7 shows that, in most cases, returns Granger cause trading volume. This phenomenon diminishes with a longer lag structure. Trading volume does not Granger cause stock returns at a $1 \%$ significance level in any case.

When we perform Granger-Causality tests for volume and volatility, see Table 5.8 , we find different results. Volatility again Granger causes volume in a large number of cases and for some stocks (AMO, HEW and the S\&P 500) volume actually Granger causes volatility. Furthermore it is an interesting feature that the $F$-statistics in both Tables for the S\&P 500 series are large relative to those for the individual stocks. It seems that Granger-Causality is (slightly) present in the stock market more at an aggregate and less at an individual level. In conclusion we can say that sizeable dynamic effects related to trading volume seem to be present in the VAR systems we analysed.

Summarizing: the trivariate VAR models do not give us any economic rationale about why trading volume should be incorporated. They merely indicate that volume is an important variable in a model that tries to describe the way stock returns and return volatility behave. A nonlinear framework might be a fruitful way to proceed, see for instance Gallant, Rossi and Tauchen (1992) and LeBaron (1993). We will continue with the construction of various models in which trading volume is a scale factor for conditional volatility, both in a Stochastic Volatility and a (E)GARCH setting. 
Table 5.7 Granger Causality Tests: Volume and Retums

\begin{tabular}{|c|c|c|c|c|c|c|}
\hline & ALC & AMO & BRI & CHA & HEW & $58 P 500$ \\
\hline$F(1) R V$ & $9.14^{*}$ & $8.36^{\circ}$ & 2.68 & $11.40^{\circ}$ & 0.13 & $57.77^{\circ}$ \\
\hline$F(1) \cup R$ & 0.51 & 0.01 & 0.70 & 5.98 & 0.00 & 0.01 \\
\hline $\mathrm{F}(2) \mathrm{RV}$ & $6.00^{\circ}$ & $6.13^{\circ}$ & 2.73 & $5.46^{\circ}$ & 0.03 & $84.38^{\circ}$ \\
\hline$F(2) \vee R$ & 0.42 & 0.32 & 0.49 & 3.5 .1 & 0.35 & 1.78 \\
\hline$F(3) R V$ & $5.16^{\circ}$ & $5.64^{\circ}$ & 2.13 & $3.94^{\circ}$ & 0.01 & $51.49^{\circ}$ \\
\hline$F(3) \cup R$ & 0.28 & 0.28 & 0.34 & 2.62 & 0.66 & 1.24 \\
\hline$F(4), R V$ & $4.32^{*}$ & $4.59^{\circ}$ & 1.95 & 3.25 & 0.05 & $45.30^{\circ}$ \\
\hline$F(4) W R$ & 1.03 & 0.33 & 0.35 & 2.09 & 1.02 & 0.95 \\
\hline$F(5) R W$ & $4.14^{\circ}$ & $387^{*}$ & 1.53 & 2.68 & 0.15 & $38.93^{\circ}$ \\
\hline$F(5)$ VR & 0.87 & 0.26 & 0.28 & 2.27 & 1.00 & 2.10 \\
\hline E(10) RV & $2.45^{\circ}$ & 2.21 & 2.20 & 1.81 & 0.57 & $20.83^{\circ}$ \\
\hline$F(10)$ VR & 0.83 & 0.46 & 0.31 & 1.37 & 0.87 & 1.08 \\
\hline$F(20) R V$ & $2.08^{\circ}$ & 1.88 & 1.63 & 1.51 & 0.51 & $11.20^{\circ}$ \\
\hline$F(20)$ VR & 0.74 & 0.81 & 0.56 & 1.27 & 0.83 & 1.21 \\
\hline F(50) RV & 1.42 & 1.14 & 1.07 & 1.31 & 0.67 & $5.19^{\circ}$ \\
\hline$F(50)$ VR & 0.75 & 1.13 & 0.80 & 1.40 & 0.83 & 1.36 \\
\hline
\end{tabular}

$F(p)$ denotes the F-statistic for $p$ lags. VR denotes the nuill hypothesis: Volumes do not Granger cause Returns; RV denotes the null hypothesis: Returns do not Granger cause Volume. "denotes statistical significance at the $1 \%$ level.

Table 5.8 Granger-Causality Tests: Volume and Volatility

\begin{tabular}{|c|c|c|c|c|c|c|}
\hline & $\mathrm{ALC}$ & $\mathrm{AMO}$ & BRI & $\mathrm{CHA}$ & HEW & $58 \mathrm{P} 500$ \\
\hline$F(1) R 2 V$ & $10.58^{\circ}$ & $20.57^{\circ}$ & 3.15 & 2.59 & $40.24^{*}$ & 2.33 \\
\hline$F(1) V R 2$ & 2.69 & $22.36^{\circ}$ & 0.03 & 0.37 & $26.27^{\circ}$ & $11.02^{\prime \prime}$ \\
\hline$F(2) R 2 V$ & $6.20^{\circ}$ & $16.90^{\circ}$ & $16.68^{\circ}$ & $5.89^{*}$ & $21.79^{\circ}$ & $106.79^{\circ}$ \\
\hline$F(2) \vee R 2$ & 1.86 & $7.15^{*}$ & 0.33 & 1.04 & $15.99^{*}$ & $7.18^{\circ}$ \\
\hline$F(3) R 2 V$ & $5.38^{m}$ & $12.89^{\circ}$ & $11.82^{\circ}$ & 5.88 & $13.95^{\circ}$ & $59.06^{\circ}$ \\
\hline$F(3) \cup R 2$ & 1.83 & $4.04^{*}$ & 1.77 & 1.25 & $9.07^{*}$ & $431^{\circ}$ \\
\hline$F(4) R 2 V$ & $4.46^{\prime \prime}$ & $10.99^{\circ}$ & $10.62^{\circ}$ & $4.63^{*}$ & $11.37^{\prime \prime}$ & $41.66^{\circ}$ \\
\hline$F(4)$ VR2 & 1.69 & 2.64 & 2.29 & 1.41 & $7.15^{*}$ & 3.30 \\
\hline$F(5) R Z V$ & $3.80^{\circ}$ & $8.87^{\circ}$ & $10.18^{\circ}$ & $3.78^{\circ}$ & $9.04^{*}$ & $31.20^{\circ}$ \\
\hline$F(5) \vee R 2$ & 1.53 & 1.80 & 1.61 & 2.74 & $5.19^{\prime \prime}$ & 2.56 \\
\hline F(10) R2V & 2.13 & $4.72^{\circ}$ & $5.60^{\circ}$ & 1.80 & $5.55^{\circ}$ & $15.47^{\circ}$ \\
\hline$F(10)$ WR2 & 1.45 & 2.03 & 1.61 & $2.42^{*}$ & $2.41^{\circ}$ & 1.44 \\
\hline$F(20) R 2 V$ & 1.63 & $3.12^{\circ}$ & $3.25^{\circ}$ & 1.15 & $3.31^{\circ}$ & $8.29^{\prime \prime}$ \\
\hline$F(20)$ VR2 & 1.39 & 1.17 & 1.53 & $2.16^{\circ}$ & 1.64 & 1.04 \\
\hline $\mathrm{F}(50) \mathrm{RZV}$ & 1.11 & $1.88^{\circ}$ & $1.89^{\circ}$ & 0.95 & $1.75^{\circ}$ & $3.94^{\circ}$ \\
\hline$F(50)$ WR2 & 1.11 & 0.97 & 0.99 & $1.54^{*}$ & 1.18 & 0.90 \\
\hline
\end{tabular}

$F(p)$ denotes the F-statistic for $p$ lags. VRZ denotes the null hypothesis: Volumes do not Granger cause Volatility; R2V denotes the null hypothesis: Volatility does not Granger cause Volume. "denotes statistical significance at the $1 \%$ level. 


\subsection{Estimation Results}

In this section we will first present estimation results for the Stochastic Volatility models mentioned in sections 5.2 and 5.3. For reasons of comparison with results in the previous chapters we will furthermore include GARCH and EGARCH estimation results for this data set ${ }^{20}$. Like Harvey and Shephard (1994) we estimate all models on the residuals of an $\mathrm{AR}(1)$ of $y_{i}$ in order to remove any first-order serial correlation from the return series. In Tables 5.9 through 5.12 we provide QML estimation results for the various SV models. We start our presentation in Table 5.9 with the QML estimation results of a basic SV model without the leverage effect and trading volume incorporated in the model. Results are in concordance with typical results from financial time series estimated in a SV framework: high persistence in the volatility $(\varphi)$ and a low signal-to-noise ratio $\left(\sigma_{\eta}{ }^{2} / \sigma_{\xi}{ }^{2}\right)$. Note that all estimates of $\varphi$ and $\sigma_{\eta}{ }^{2}$ are statistically significant at the $1 \%$ level. Including the leverage effect in the basic model through $\rho$, see equations $5.30-5.32^{21}$, leads to rather mixed results. In particular, see Table 5.10, only two series (S\&P 500 and Bristol Myers) exhibit a significant leverage effect $(\rho)$. Tables 5.11 and 5.12 show results after including trading volume in the measurement equation with and without incorporating the leverage effect respectively. Note that $\lambda_{y}$ is statistically significant at the $1 \%$ level for all series in both specifications. Significant leverage effects are found in three cases now, but changes in the leverage parameter between the specifications without and with leverage are not of the same sign, i.e. including trading volume does not influence leverage parameters in a uniform way. Incorporating trading volume clearly enhances the fit, measured by the log-likelihood, of the basic SV model significantly. In every case likelihood ratio statistics are larger than the critical value 6.63 at a $1 \%$ significance level. The persistence in volatility is not influenced by including trading volume in the transition equation.

The main conclusion of these four Tables is obvious: trading volume is an important variable in a SV model. In addition we computed several diagnostic test statistics using one-step ahead standardized prediction errors from the models. The Ljung-Box test statistics show that for some series the AR(1) dynamic specification for the conditional variance is not sufficient. Furthermore, kurtosis remains high. The sign bias tests show that sign effects are in general not present in the one-step ahead prediction errors of the Kalman filter.

20. The multiplicative GARCH and EGARCH models in Chapter 4 serve as a first benchmark here.

21 Here $\lambda_{y}$ is restricted to zero. 
Table 5.9 A Basic Stochastic Volatility Model

\begin{tabular}{lcccccc}
\hline & ALC & ANO & BRI & CHA & HEN & S\&P 500 \\
\hline$B_{y}$ & -0.666 & -0.865 & -0.757 & -0.303 & -0.316 & -1.335 \\
& $(0.358)$ & $(1.995)$ & $(0.254)$ & $(0.136)$ & $(0.365)$ & $(0.642)$ \\
$\varphi$ & 0.982 & 0.995 & 0.973 & 0.916 & 0.974 & 0.992 \\
& $(0.003)$ & $(0.007)$ & $(0.002)$ & $(0.004)$ & $(0.002)$ & $(0.004)$ \\
$\sigma_{n i}$ & 0.130 & 0.082 & 0.163 & 0.340 & 0.190 & 0.085 \\
& $(0.004)$ & $(0.004)$ & $(10.006)$ & $(0.014)$ & $(0.006)$ & $(0.002)$ \\
Log L & -12017 & -11951 & -12070 & -12264 & -12237 & -11691 \\
\hline Min & -8.409 & -5.557 & -6.555 & -5.831 & -8.183 & -5.95 \\
Max & 2.712 & 3.110 & 2.853 & 2.481 & 2.688 & 3.096 \\
Skew & -1.264 & -1.221 & -1.365 & -1.280 & -1.542 & -1.286 \\
Kurt & 6.155 & 5.430 & 6.078 & 5.451 & 7.624 & 5.726 \\
LB(10) & 5.340 & 19.677 & 25.070 & 15.045 & 19.153 & 16.770 \\
LB (20) & 16.370 & 27.261 & 38.373 & 23.744 & 28.647 & 28.785 \\
SBT & 0.865 & -0.678 & 0.968 & 1.529 & 1.253 & 1.068 \\
NSBT & -1.507 & 1.694 & -1.813 & 1.800 & 0.098 & -0.052 \\
PSBT & 0.009 & -0.102 & -1.024 & -1.795 & -0.840 & -0.191 \\
JSBT & 3.066 & 3.834 & 3.552 & 4.511 & 2.684 & 2.468 \\
\hline
\end{tabular}

Heteroskedasticity-consistent standard errors are given in parentheses. Min, Max, Skew and Kurt are the minimum, maximum, skewness and kurtosis, respectively, af the standardized one-step prediction errors. $L B$ (p) denotes the Ljung-Box statistics with p lags. SBT, NSBT, PSBT and ISBT are sign bias test (see text). See Table 5.1 for a description of series.

Table 5.10 Stochastic Volatility with Leverage

\begin{tabular}{lcccccc}
\hline & ALC & AMO & BRI & CHA & HEW & S\&P 500 \\
\hline$\beta_{y}$ & -0.661 & -0.879 & -0.781 & -0.307 & -0.327 & -1.313 \\
& $(0.350)$ & $(1.898)$ & $(0.282)$ & $(0.144)$ & $(0.399)$ & $(0.393)$ \\
$p$ & 0.981 & 0.995 & 0.977 & 0.924 & 0.977 & 0.986 \\
& $(0.005)$ & $(0.001)$ & $(0.006)$ & $(0.033)$ & $(0.006)$ & $(0.003)$ \\
$\sigma_{n}$ & 0.132 & 0.081 & 0.152 & 0.322 & 0.176 & 0.120 \\
& $(0.020)$ & $(0.010)$ & $(0.023)$ & $(0.093)$ & $(0.029)$ & $(0.018)$ \\
$\rho$ & 0.070 & -0.023 & -0.307 & -0.096 & -0.113 & -0.481 \\
& $(0.121)$ & $(0.260)$ & $(0.109)$ & $(0.067)$ & $(0.092)$ & $10.149)$ \\
Log L & -12017 & -11951 & -12065 & -12263 & -12236 & -11680 \\
\hline Min & -8.404 & -5.547 & -6.619 & -5.861 & -8.283 & -5.961 \\
Max & 2.720 & 3.109 & 2.874 & 2.454 & 2.680 & 2.970 \\
SKew & -1.264 & -1.221 & -1.368 & -1.283 & -1.544 & -1.305 \\
Kurt & 6.159 & 5.43 & 6.092 & 5.461 & 7.642 & 5.770 \\
LB(10) & 5.209 & 19.990 & 24.977 & 1.4 .770 & 18.391 & 19.398 \\
LB (20) & 15.768 & 27.517 & 38.373 & 23.014 & 38.503 & 31.033 \\
SBT & 0.712 & -0.704 & 0.938 & 1.356 & 1.241 & 1.126 \\
NSBT & -1.541 & 1.690 & -1.692 & -1.762 & 0.089 & -0.210 \\
PSBT & 0.013 & -0.099 & -0.931 & -1.751 & -0.796 & -0.366 \\
JSBT & 3.016 & 3.827 & 3.019 & 4.460 & 2.615 & 2.072 \\
\hline
\end{tabular}

Heteroskedasticity-consistent standard errors are given in parentheses. Min, Max, Skew and Kurt are the minimum, maximum, skewness and kurtosis, respectively, of the standardized one-step prediction errors. LB (o) denotes the Ljung-Box statistics with $p$ lags. SBT, NSBT, PSBT and JSBT are sign bias test (see tex1). See Table 5.1 for a description of series. 
Table 5.11 Stochastic Volatility and Volume

\begin{tabular}{|c|c|c|c|c|c|c|}
\hline & ALC & AMO & BRI & CHA & HEV & S8P 500 \\
\hline $\boldsymbol{B}_{y}$ & $\begin{array}{c}1.594 \\
(0.396)\end{array}$ & $\begin{array}{c}-3.263 \\
(1.650)\end{array}$ & $\begin{array}{r}2.814 \\
(0.505)\end{array}$ & $\begin{array}{r}-1.980 \\
(0.275)\end{array}$ & $\begin{array}{l}-2.215 \\
(0.587)\end{array}$ & $\begin{array}{r}-11.014 \\
(1.997)\end{array}$ \\
\hline$\varphi$ & $\begin{array}{c}0.983 \\
(0.004)\end{array}$ & $\begin{array}{c}0.995 \\
(0,002)\end{array}$ & $\begin{array}{c}0.985 \\
(0,002)\end{array}$ & $\begin{array}{r}0.957 \\
(0.008)\end{array}$ & $\begin{array}{c}0.989 \\
(0.003)\end{array}$ & $\begin{array}{r}0.994 \\
(0.001)\end{array}$ \\
\hline$a_{r_{n}}$ & $\begin{array}{l}0.125 \\
(0.018)\end{array}$ & $\begin{array}{c}0.077 \\
(0.008)\end{array}$ & $\begin{array}{c}0.125 \\
(0.010)\end{array}$ & $\begin{array}{r}0.229 \\
(0.028)\end{array}$ & $\begin{array}{c}0.104 \\
(0.015)\end{array}$ & $\begin{array}{c}0.092 \\
(0.004)\end{array}$ \\
\hline $\log L$ & $\begin{array}{c}0.152 \\
(0.029) \\
-11998\end{array}$ & $\begin{array}{c}0.366 \\
(0.035) \\
-11855\end{array}$ & $\begin{array}{c}0.331 \\
(0.033) \\
-11992\end{array}$ & $\begin{array}{c}0.282 \\
(0.029) \\
-12193\end{array}$ & $\begin{array}{c}0.312 \\
(0.027) \\
-12121\end{array}$ & $\begin{array}{c}1.160 \\
(0.153) \\
-11626\end{array}$ \\
\hline Min & -8.500 & -5.545 & -6.438 & -6.147 & -8.322 & -5.984 \\
\hline Max & 2.578 & 2.726 & 2.330 & 2.086 & 2.458 & 2.162 \\
\hline Skew & -1.284 & -1.295 & -1.423 & -1.324 & -1.610 & -1.338 \\
\hline Kurt & 6.239 & 5.667 & 6.210 & 5.571 & 7.83 .4 & 5.798 \\
\hline$L E(10)$ & 5.433 & 11.900 & 18.845 & 7.997 & 10.572 & 17.127 \\
\hline$\angle B(20)$ & 18.007 & 19.199 & 33.877 & 18.091 & 29.227 & 25.599 \\
\hline SET & 0.492 & -0.478 & 1.627 & 2.496 & 0.306 & 1.884 \\
\hline NSBT & -1.627 & 1.615 & -1.769 & -1.132 & 0.024 & -0.023 \\
\hline PSBT & -0.014 & -0.064 & -1.509 & -1.616 & -0.167 & -0.818 \\
\hline ISBT & 3.322 & 6.612 & 3.840 & 6.493 & 0.167 & 6.410 \\
\hline
\end{tabular}

Table $\mathbf{5 . 1 2}$ Stochastic Volatility and Volume with Leverage

\begin{tabular}{|c|c|c|c|c|c|c|}
\hline & $\mathrm{ALC}$ & AMO & $\mathrm{BRI}$ & $\mathrm{CHA}$ & HEW & S\&P 500 \\
\hline$\beta_{y}$ & $\begin{array}{l}-1.595 \\
(0.399)\end{array}$ & $\begin{array}{l}-3.306 \\
(1.731)\end{array}$ & $\begin{array}{l}-2.848 \\
(0.510)\end{array}$ & $\begin{array}{l}-2.010 \\
(0.288)\end{array}$ & $\begin{array}{l}-2.209 \\
(0.650)\end{array}$ & $\begin{array}{r}-11.505 \\
(1.285)\end{array}$ \\
\hline$\phi$ & $\begin{array}{c}0.983 \\
(0.004)\end{array}$ & $\begin{array}{c}0.996 \\
(0.001)\end{array}$ & $\begin{array}{c}0.986 \\
(0.002)\end{array}$ & $\begin{array}{c}0.962 \\
(0.007)\end{array}$ & $\begin{array}{c}0.990 \\
(0.003)\end{array}$ & $\begin{array}{c}0.985 \\
(0.005)\end{array}$ \\
\hline$\sigma_{n}$ & $\begin{array}{c}0.125 \\
(0.018)\end{array}$ & $\begin{array}{c}0.074 \\
(0.006)\end{array}$ & $\begin{array}{c}0.125 \\
(0.011)\end{array}$ & $\begin{array}{c}0.213 \\
(0.027)\end{array}$ & $\begin{array}{c}0.096 \\
(0.014)\end{array}$ & $\begin{array}{c}0.127 \\
(0.021)\end{array}$ \\
\hline$p$ & $\begin{array}{c}0.015 \\
(0.128)\end{array}$ & $\begin{array}{l}-0.136 \\
(0.303)\end{array}$ & $\begin{array}{l}-0.338 \\
(0.135)\end{array}$ & $\begin{array}{c}-0.199 \\
(0.082)\end{array}$ & $\begin{array}{r}-0.185 \\
(0.116)\end{array}$ & $\begin{array}{c}-0.514 \\
(0.124)\end{array}$ \\
\hline$A_{y}$ & $\begin{array}{c}0.152 \\
(0.029)\end{array}$ & $\begin{array}{c}0.366 \\
(0.035)\end{array}$ & $\begin{array}{c}0.331 \\
(0.033)\end{array}$ & $\begin{array}{c}0.285 \\
(0.029)\end{array}$ & $\begin{array}{c}0.311 \\
(0.027)\end{array}$ & $\begin{array}{r}1.228 \\
(0.155)\end{array}$ \\
\hline $\log L$ & -11998 & -11855 & -11987 & -12190 & -12120 & -11604 \\
\hline Mins & .8 .499 & -5.565 & .6 .493 & -5.898 & -8.350 & -5.972 \\
\hline Max & 2579 & 2.706 & 2.345 & 2.390 & 2.455 & 1.979 \\
\hline Skew & -1.284 & -1.296 & -1.426 & -1.330 & -1.613 & -1.365 \\
\hline Kurt & 6240 & 5.667 & 6.226 & 5.597 & 7.853 & 5.881 \\
\hline LB $(10)$ & 5.431 & 12.326 & 18.660 & 7.101 & 10.492 & 18.855 \\
\hline $18(20)$ & 17.985 & 19.658 & 33.627 & 17.994 & 28.762 & 28.374 \\
\hline $5 \mathrm{BT}$ & 0.509 & 0.575 & 1.375 & 2.212 & 0.564 & 1.608 \\
\hline NSBT & -1.633 & 1.623 & -1.643 & -1.483 & 0.015 & -0.283 \\
\hline PSBT & -0.013 & -0.094 & -1.489 & -1.382 & -0.171 & -0.944 \\
\hline JSBT & 3.341 & 7.359 & 3.436 & 4.994 & 0.625 & 3.563 \\
\hline
\end{tabular}

Notes for tables 5.11 and 5.12: Heteroskedasticity-consistent standard errors are given in parentheses. Min, Max, Skew and Kurt are the minimum, maximum, skewness and kurtosis, respectively, of the standardized one-step prediction errors. LB ( $p$ ) denotes the Ljung-Box statistics with plags. SBT, NSBT, PSBT and ISBT are sign bias test (see text). See Table 5.11 for a description of series. 
As mentioned in the previous sections, we propose to use trading volume as a scale factor for volatility. In Chapter 4 we presented a multiplicative GARCH model, where volume was interpreted as a scale factor too. For reasons of comparison we decided to include estimation results for a multiplicative GARCH and EGARCH model. The multiplicative GARCH model we estimate has the following specification:

$y_{t}=\mu+\varepsilon_{i}, \quad \varepsilon_{t}-N\left(0, h_{t}\right)$

$h_{t}=\beta_{0} V_{t}^{2 \gamma}+\left(\frac{V_{t}}{V_{t-1}}\right)^{2 \gamma}\left(\beta_{1} \varepsilon_{t-1}^{2}+\beta_{2} h_{t-1}\right)$

This model is motivated by earlier work on interest rates by Koedijk, Nissen, Schotman and Wolff (1994) and Brenner, Harjes and Kroner (1993) ${ }^{22}$. Table 5.13 presents GARCH estimation results for the Gallant, Rossi and Tauchen (1992) and Lamoureux and Lastrapes (1994) data set. The parameter $y$ that is related to the volume variables is significant in every case with the exception of Champion. Note that the persistence of conditional volatility, measured by the sum of $\beta_{1}$ and $\beta_{2}$, remains thigh. This contrasts sharply with results in Lamoureux and Lastrapes (1990a) ${ }^{23}$. We therefore conclude again that the high persistence in volatility may be interpreted as evidence that trading volume may be interrelated to returns and volatility in a more complicated way than just a linear relationship. These results confirm the investigation into a multiplicative relationship between volume and volatility in Chapter 4 . In order to capture possible leverage effects in GARCH-like models we also estimated an EGARCH model, see Nelson (1991). The multiplicative EGARCH specification we use has the following conditional variance equation:

$\ln \sigma_{t}^{2}=\omega+\varphi \varepsilon_{t-1}+\delta\left(\left|\varepsilon_{t-1}\right|-\sqrt{\frac{2}{\pi}}\right)+\beta \ln \sigma_{t-1}^{2}$

The results in Table 5.14 show that high persistence in volatility is confirmed here also. The total leverage effect in this specification is equal to $\varphi / \delta$, see Taylor (1994) and corroborates the findings on the leverage effect in the E(GARCH) estimation results of Chapter 4.

22 See Chapter 4 for a detailed discussion on the derivation of this model specification.

${ }^{23}$ It should be noted that Lamoureux and Lastrapes (1990a) use an additive model, which leads to a decrease in persistence of conditional volatility. In Chapter 4 we saw that this was also the case for the German data set. 
Table 5.13 Multiplicative GARCH and Volume Results

\begin{tabular}{lcccccc}
\hline & ALC & AMO & BRI & CHA & HEW & S\&P \\
\hline$H^{*}$ & 0.024 & 0.033 & 0.050 & 0.001 & 0.025 & -0.004 \\
$B_{0}$ & $(0.022)$ & $(0.024)$ & $(0.028)$ & $(0.047)$ & $(0.016)$ & $(0.009)$ \\
& 0.061 & 0.036 & 0.173 & 0.213 & 0.219 & 0.053 \\
$B_{1}$ & $(0.025)$ & $(0.014)$ & $(0.078)$ & $(0.109)$ & $(0.072)$ & $(0.016)$ \\
& 0.060 & 0.076 & 0.084 & 0.078 & 0.072 & 0.077 \\
$B_{2}$ & $(0.014)$ & $(0.017)$ & $(0.020)$ & $(0.024)$ & $(0.013)$ & $(0.077)$ \\
& 0.928 & 0.922 & 0.892 & 0.900 & 0.910 & 0.923 \\
& $(0.017)$ & $(0.016)$ & $(0.028)$ & $(0.033)$ & $(0.016)$ & $(0.006)$ \\
& 0.081 & 0.122 & 0.078 & 0.041 & 0.140 & 0.678 \\
Log L & $(0.013)$ & $(0.018)$ & $(0.015)$ & $(0.058)$ & $(0.011)$ & $(0.039)$ \\
& -9787 & -8864 & -9295 & -10739 & -10440 & -7261 \\
\hline
\end{tabular}

Table 5.14 Multiplicative EGARCH Riesults

\begin{tabular}{lcccccc}
\hline & ALC & AMO & BRI & CHA & HEW & S\&P \\
\hline$\omega$ & 0.039 & 0.014 & 0.066 & 0.052 & 0.034 & 0.000 \\
& $(0.014)$ & $(0.005)$ & $(0.025)$ & $(0.017)$ & $(0.009)$ & $(0.001)$ \\
& 0.003 & -0.024 & -0.025 & -0.039 & -0.025 & -0.074 \\
$\delta$ & $(0.006)$ & $(0.012)$ & $(0.020)$ & $(0.013)$ & $(0.009)$ & $(0.007)$ \\
& 0.179 & 0.155 & 0.235 & 0.169 & 0.146 & 0.119 \\
$B$ & $(0.032)$ & $(0.031)$ & $(0.044)$ & $(0.031)$ & $(0.021)$ & $(0.010)$ \\
& 0.974 & 0.990 & 0.952 & 0.971 & 0.983 & 0.990 \\
$\gamma$ & $(0.012)$ & $(0.003)$ & $(0.019)$ & $(0.010)$ & $(0.005)$ & $(0.002)$ \\
& 0.084 & 0.172 & 0.157 & 0.127 & 0.137 & 0.721 \\
& $(0.014)$ & $(0.020)$ & $(0.018)$ & $(0.013)$ & $(0.010)$ & $(0.036)$ \\
$\log \mathrm{L}$ & 0.017 & -0.155 & -0.106 & -0.231 & -0.171 & -0.622 \\
\hline
\end{tabular}

Notes for tables 5.13 and 5.14: Heteroskedasticity consistent standard errors are given in parentheses. see Table 5.1 for description of series.

The (E)GARCH results here confirm the stochastic volatility results made earlier. Trading volume seems to enhance the fit and for some stocks there is evidence of a leverage effect.

\subsection{Interpretation of Results and Concluding Comments}

In this chapter we performed an empirical analysis of the relationship between stock return volatility and trading volumes. Summary statistics, several sign bias tests, Granger Causality tests and VAR estimations clearly demonstrated that there is a strong dependence between stock returns, return volatility and trading volume. This dependence is not necessarily of a linear nature, like for instance in the VAR models and therefore we shifted our attention to Stochastic Volatility and (E)GARCH models. We showed that a Stochastic Volatility model naturally arises when we assume that returns are generated by the mixture of distributions hypothesis (MDH). A natural implication of the SV model we 
derive, is that daily trading volume can act as a time-varying scale factor for conditional volatility. The model we estimate eventually, explicitly incorporates daily trading volume in the measurement equation of a univariate SV model. Our empirical results are based on a sample of five individual stocks listed on the New York Stock Exchange and the S\&P 500 composite stock index.

All models were estimated by Quasi Maximum Likelihood estimation. Results show that including volume leads to a much better fit of the models, implying that trading volume is a significant scale factor for the conditional volatility: the role we hypothesized for trading volume is empirically confirmed. We do not find, however, that the persistence of conditional volatility diminishes once volume has been added to the model. This confirms the results of Chapter 4 where we conducted the same analysis using a GARCH framework. It may be that trading volume interacts with stock returns in a more diverse way. No conclusive evidence was found for the leverage effect, although some simple tests show that it is predominantly a tail phenomenon. Only for the S\&P 500 index we find a significant leverage effect that is much less dominated by a few outlying observations.

It should be noted that the point that sets the analysis in Chapters 4 and 5 apart from previous univariate studies in this area, is the introduction of contemporaneously observed trading volume in a multiplicative manner. The results are generally encouraging: both in the Harvey, Ruiz and Shephard (1994) stochastic volatility models with a multiplicative (log-additive) volume term and the (E)GARCH model with multiplicative volume, we obtain the result that the volume coefficient is unanimously, highly significant. Furthermore we clearly showed that, in contrast to the main findings of Lamoureux and Lastrapes (1990a), the estimated volatility persistence measures remain very high after the introduction of the volume component in the specification.

Analogous to our GARCH estimation results in Chapter 4 we should be cautious in interpreting the univariate estimation results. The findings need to be digested in further research projects and translated into reasonable hypotheses regarding the structure of the joint system, and thus serve as inspiration for further, more structurally based explorations of these important issues in finance. Furthermore we know that the mixture hypothesis explicitly points to a bivariate specification: both trading volume and volatility are influenced simultaneously, under various assumptions, by the same unobservable information flow. Andersen (1996) states that we need a bivariate system specification, if we want to understand the relationship between return volatility and trading volume. The evidence in the bivariate VAR analysis so far is however inconclusive. We therefore shift our attention in the next chapters to bivariate specifications with a strong link to the existing $\mathrm{MDH}$ literature. 


\section{Appendix A Impulse Response Functions}

\section{Volatility and Volume}
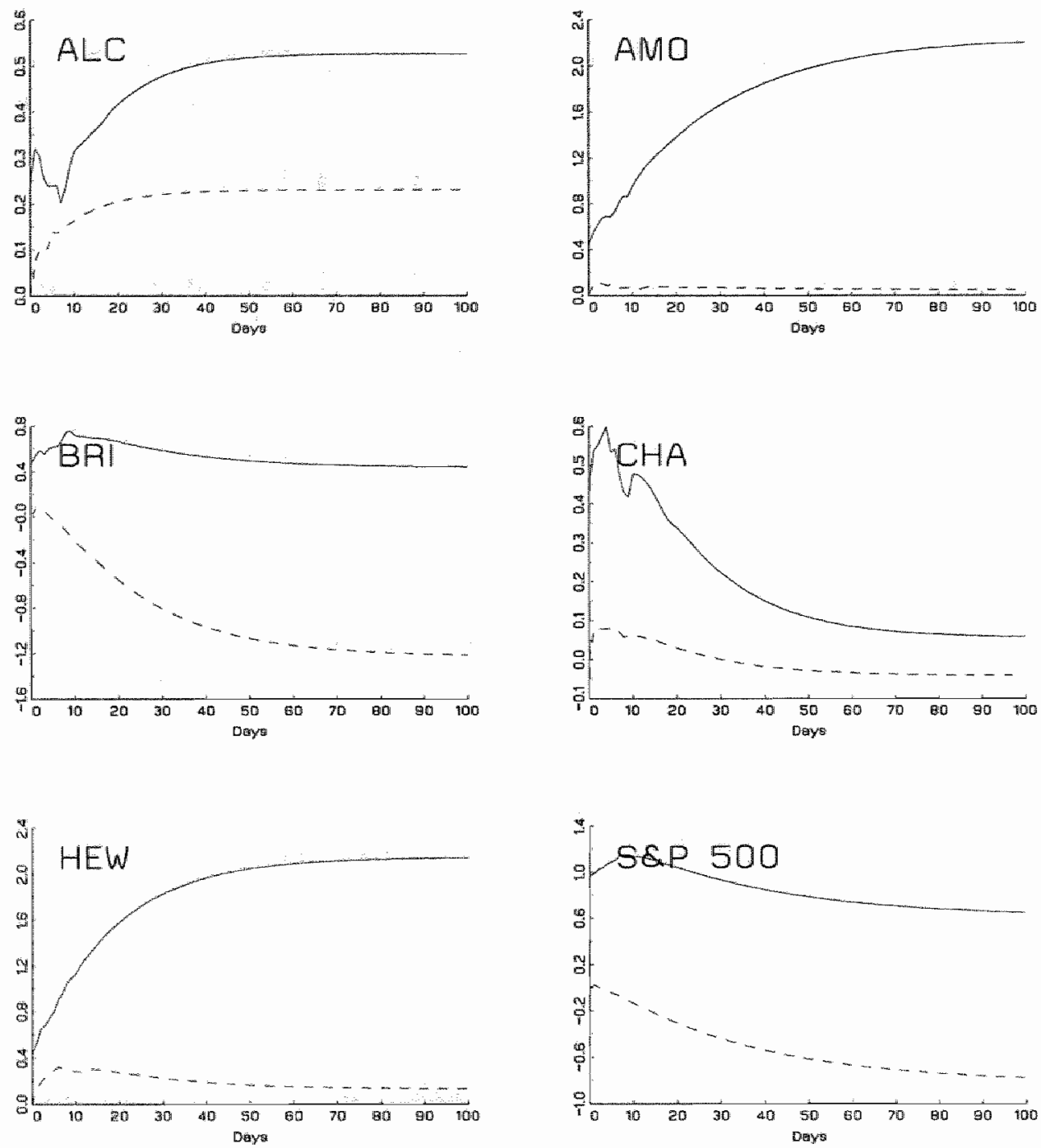

Figure 5.2 IRF functions: the response of volume to a shock in volatility (dashed) and the response of volatility to a shock in volume (solid). See text for a description of the graphs. 

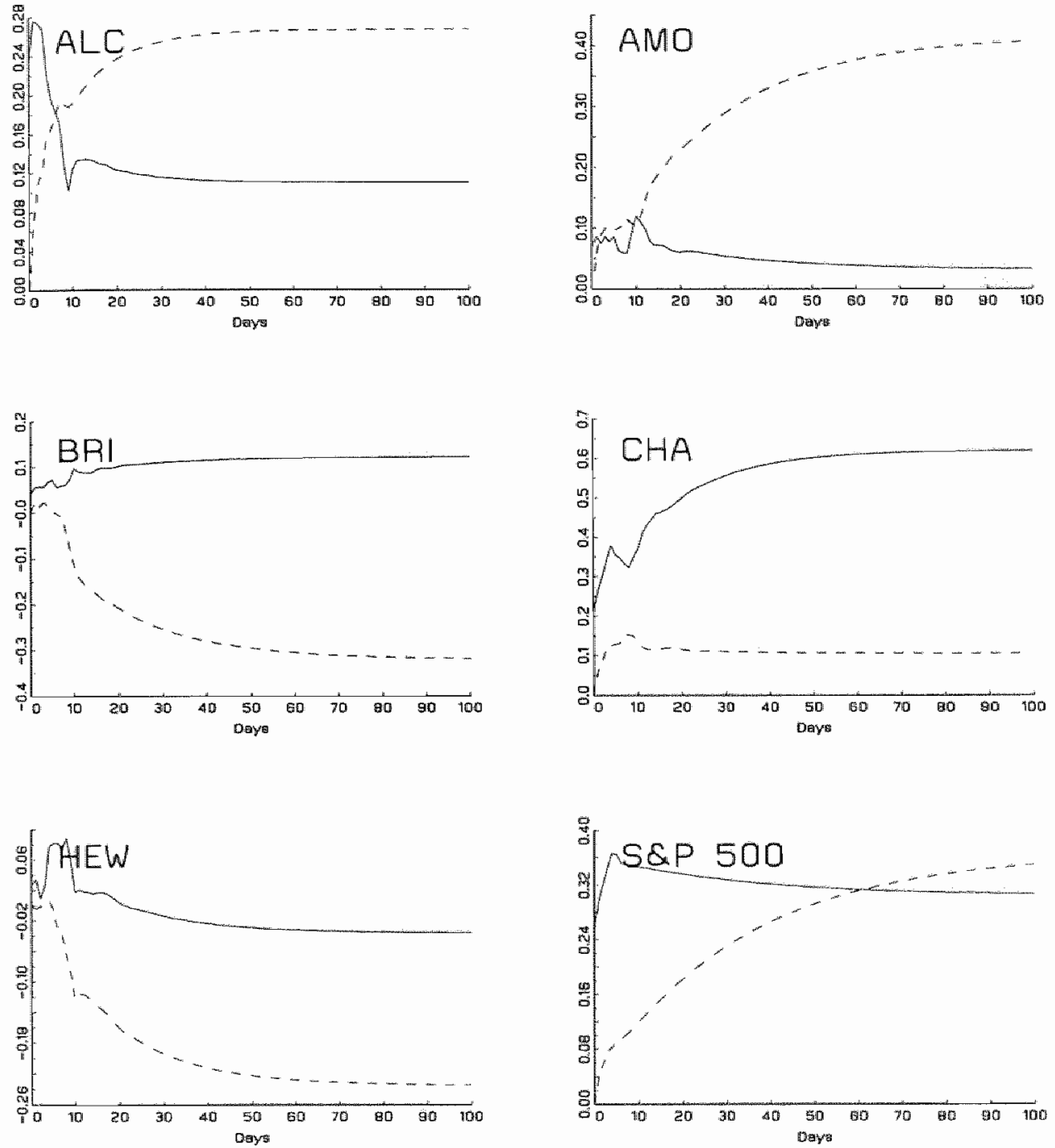

Figure 5.3 IRF functions: the response of volume to a shock in returns (dashed) and the response of returns to a shock in volume (solid). See text for a description of the graphs. 


\section{CHAPTER 6}

\section{Volatility and Volume: an Intra-Daily Perspective}

\subsection{Introduction}

In the previous chapters we explicitly proposed to give trading volume a role as scale factor for conditional volatility. In the empirical analysis so far we used daily return and volume series. The main conclusion is that there is definitely a strong link between trading volume and (conditional) volatility. Granger Causality tests and impulse response functions of estimated VAR-models in the previous chapter furthermore indicated that trading volume probably plays an important role in the disequilibrium dynamics of financial markets. An interesting question in this chapter is whether this phenomenon can be confirmed for intra-daily stock return and volume data. This is particularly interesting as the financial literature pays a lot of attention to market microstructure related models that try to explain the relationship between both variables during and across days, see for instance Kyle (1985), Admati and Pfleiderer (1988 and 1989) and Foster and Viswanathan $\left(1990,1991,1993 \mathrm{a}\right.$ and 1993b) ${ }^{1}$. The main objective is however to present and estimate various models, both univariate for hourly price changes and bivariate for hourly price changes and hourly trading volume.

In the first part of this chapter we extend our analysis of daily price and volume data by investigating whether trading volume can also act as a scale factor for volatility using intra-daily data. In the second part we focus on several mixture models, introduced by Clark (1973) and discussed and further developed by Tauchen and Pitts (1983) (TP83) and Harris (1987). These approaches all have in common that they assume that the latent information variable, influencing both volumes and returns, is serially independent. This is in fact not consistent with the observed dynamics of financial markets, see Taylor (1994) for an overview article and Chapters 3 through 5 . In the empirical sections of this chapter we

1 See Chapter 2 for a list of papers on this subject. 
estimate both univariate and bivariate specifications with a serially dependent mixing variable. In addition we estimate these specifications with an independent mixing variable too, serving as a natural benchmark for the dynamic specification.

The time series characteristics of intra-daily data in general differ from data of other frequencies, because several systematic effects can play a dominant role. Jain and Joh (1988) for instance, showed for the New York Stock Exchange that there is a strong systematic effect in trading volume during the trading day. Volume tends to be high in the opening and closing hours, however most shares are traded in the opening hour. Volume declines monotonically until the fourth hour and increases again in the fifth and sixth hour. Higher volumes may occur in the first trading hour because investors transact on information gathered during the overnight period and in the hours before the stock market opens. The higher volumes in the last hour may reflect the closing or hedging of positions by investors and brokers who are not able to monitor their positions in the overnight period. Jain and Joh (1988) were able to use a data set of several years, which gave them the opportunity to present results specified for different week days. Average trading volume proved to be lowest on Monday, goes up monotonically from Monday to Wednesday, and eventually declines Thursday and Friday. This result is independent of the choice of the trading hour. In our analysis we will restrict ourselves to intra-dlaily patterns as a result of the relatively short sample period.

We start our empirical research by presenting summary statistics for the intradaily data set containing a subset of five stocks listed on the Amsterdam Stock Exchange. After adjusting the data for the systematic effects described above we first perform Granger Causality tests in order to get first insight into the link between returns, return volatility and trading volume. An important feature of this chapter is however to estimate the models presented in previous chapters of this thesis as well as the mixture specifications mentioned above. We start with the estimation of a stochastic volatility model for hourly return series using the framework discussed in Chapter 5. This estimation procedure will be extended by assuming that volume acts as a scale factor for conditional volatility in an intra-daily framework. We estimate these specifications with and without a leverage effect respectively. Subsequently, we estimate a univariate mixture model for daily price changes, introduced by Clark (1973). We will both estimate a static version of this model, where the latent mixing variable is assumed to be serially independent, and a dynamic version, where the mixing variable is a serially correlated random variable. A rather new approach in this chapter is the estimation of a bivariate stochastic volatility model for intra-daily price changes and trading volume based on the TP83 model. They use a simple 
Walrasian equilibrium framework to derive a bivariate normal distribution for trading volume and price changes conditional on the daily flow of information. The key difference here is that in contrast to the approach in previous chapters we now assume that price changes and trading volume are influenced by the latent information process simultaneously. This specification is also estimated with both a static and a dynamic version. The static models are estimated applying Maximum Likelihood techniques and equivalent to the model in Tauchen and Pitts (1983). In order to estimate the dynamic mixture models we have to solve the problem that the likelihood functions are given by high-dimensional integrals. Since these integrals cannot be solved by standard integration methods, a direct application of maximum likelihood is infeasible. We instead use a simulated maximum likelihood (SML) approach, introduced by Danielsson and Richard (1993) and further discussed in Danielsson (1994) and Liesenfeld (1996). This approach relies on Monte Carlo techniques to estimate the high-dimensional integrals.

The remainder of this chapter contains the following sections: in section 6.2 we will describe the data set and the construction of the hourly returns and trading volumes in greater detail. Furthermore we present summary statistics for returns and volumes and Granger Causality test in this section. In section 6.3 we present the univariate stochastic volatility models where trading volume acts as a scale factor. In addition, we test whether a specification incorporating a leverage effect improves results. In section 6.4 we discuss the univariate and bivariate mixture models of Clark (1973) and Tauchen and Pitts (1983). Section 6.5 presents empirical results for these models. Finally, section 6.6 concludes.

\subsection{Data Description and Summary Statistics}

The data set used in the empirical section has been provided by Beursdata, an affiliate of the Amsterdam Stock Exchange ${ }^{2}$. We received a data set containing intra-daily information on a sample of 25 stocks, all part of the well-known Amsterdam Exchange indeX (AEX). This was in fact the first stage of our stock selection procedure. In our opinion, mainly for reasons of liquidity, the other listed stocks in Amsterdam do not have the requirements for useful intra-daily research. A closer inspection of average daily trading volume and free float figures of the remaining stocks leads to the conclusion that we have to focus on a few liquid, heavily traded stocks. For that reason we decided to choose the five largest stocks of the AEX-index.

2 At this stage I would like to thank Beursdata, Martin Misseyer (Free University of Amsterdam) and Thomas Tschugmell (University of Aachen) for providing the raw data set. 

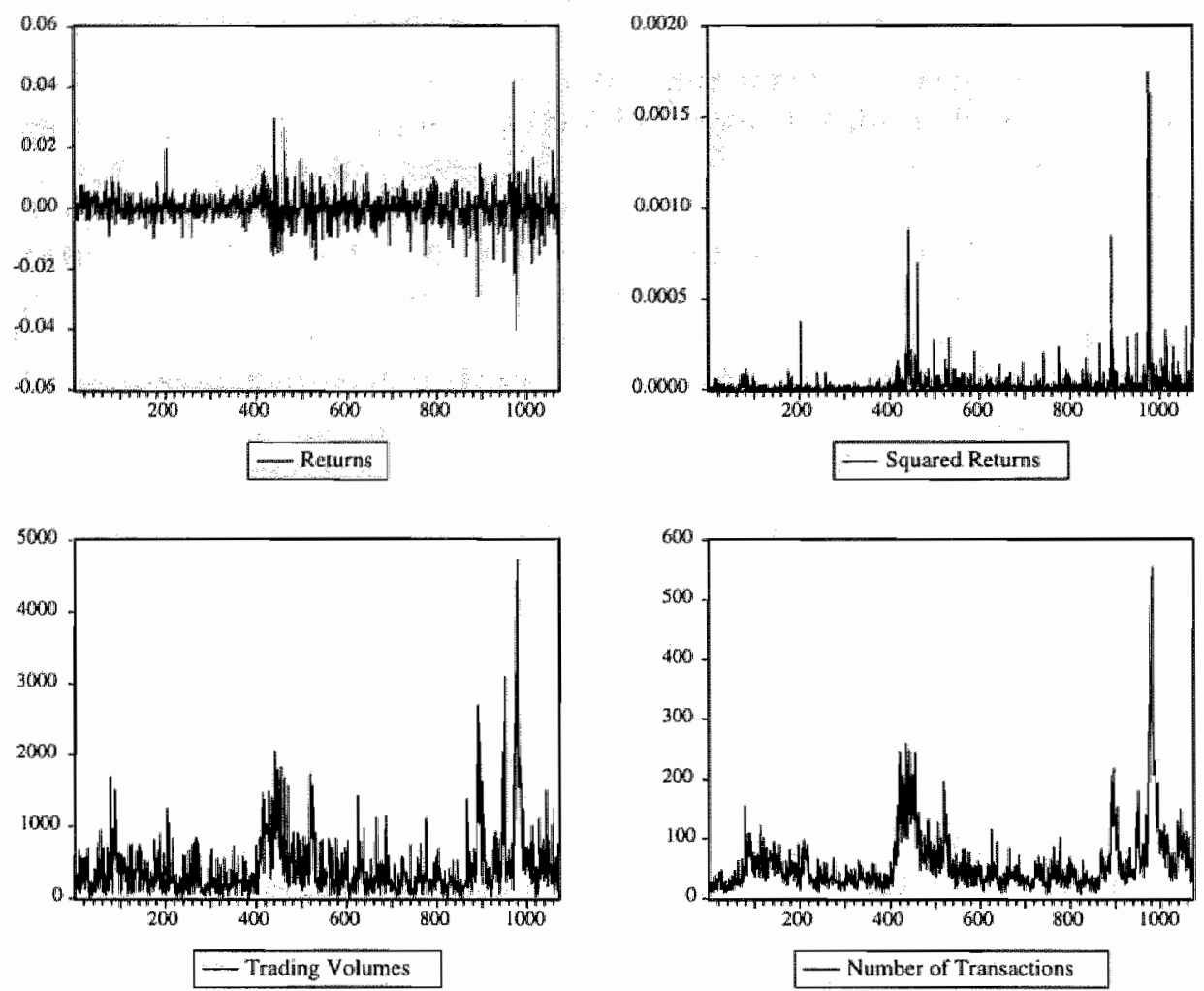

Figure 6.1 Hourly Returns, Squared Returns, Trading Volume (in thousands) and the Number of Transactions for Philips.

These stocks are respectively: $\mathrm{ABN}-\mathrm{AMRO}, \mathrm{ING}$, Philips, Royal Dutch and Unilever. ${ }^{3}$. The next step in the construction of the intra-daily data set for the empirical analysis was the choice of the time interval (in minutes). In order to avoid too much noise in the data this interval should not be too small, especially not in a relatively small stock market as the Amsterdam Stock Exchange. Following a number of intra-daily studies, we decided to use a 60-minutes interval, see for instance Jain and Joh (1988). In Appendix A we explain the construction of the 60-minutes interval data in greater detail. The stock market in Amsterdam officially opens at 9.30 a.m. and closes at 16.30 p.m., which results in seven

3 "These stocks have an aggregated market walue of more than $50 \%$ of the Dutch stock market The other stocks in the index are considerably smaller. Furthermore it should be noted that these stocks were the five largest stocks in the research period. 
hourly periods during the trading day. The period under investigation starts April 7 th, 1995, and ends November 14th, 1995. This implies a research period of more than seven months, 153 trading days with seven periods per day and 1071 observations. For each stock, hourly returns and hourly trading volumes were collected for the specified period. The return series were calculated as the logarithmic difference between hourly prices and the volume series as the number of shares traded per hour. In Figure 6.1 we graph the raw hourly returns, squared returns, trading volumes and the number of transactions for the most active stock in the sample period: Philips. The graphs clearly show the relationship between uncertainty in the stock market and activity in the stock market (trading volume) ${ }^{4}$. Furthermore we can see that the graphs of volume and the number of transactions are similar.

Table 6.1 gives a quick overview of some general features of the stocks in our sample. This table shows us that average hourly trading volume is at least 8 million guilders ( $A B N-A M R O$ ). The Philips share had a very dynamic period: despite of the fact that the aggregate market value of Royal Dutch is much larger, Philips clearly had the highest hourly turnover, trading volume and number of price changes respectively. It is clear that trading volume and the number of official price changes are heavily correlated in an intra-daily perspective. This gives us an indication that the price change process and trading volume are closely linked.

Table 6.2 presents returns, volatility (measured as squared returns) and trading volume (the number of traded shares) for the five selected stocks for every trading hour during the day. There is little evidence of systematic effects in the return series. The often quoted predictions for patterns in volatility and trading volume, see for instance Admati and Pfleiderer $(1988,1989)$, are however confirmed here. The volatility series have distinct $U$-shaped patterns where (mainly) overnight volatility and volatility in the first trading hour are high. Overnight volatility can be expected to be above average as these large stocks are listed on several stock markets across the world. The volume series exhibits a similar pattern. Here, the largest number of shares are traded in the last trading hour and clearly a $U$-shaped pattern is present during the day. As stated earlier one of the objectives of the empirical analysis in this chapter is to investigate whether the relationship between conditional volatility and trading volume, documented extensively in a daily framework in the previous chapter, can also be found on an intra-daily basis.

4 Philips announced in October 1995 that they expected profits to decrease considerably. In the trading days after this trading volume and volatility were very high. 
Table 6.1 Hourly Tumover, Trading Volume and the Number of Official Price Changes

\begin{tabular}{lccccc}
\hline & ABN-AMRO & ING & Philips & Royal Dutch & Unilever \\
\hline $\begin{array}{l}\text { Turnover }\left(10^{3}\right) \\
\text { Mean }\end{array}$ & 8757 & 8763 & 29054 & 20982 & \\
Maximum & 114000 & 127000 & 284000 & 174000 & 11368 \\
Minimum & 91 & 178 & 242 & 386 & 385 \\
& & & & & \\
Volume $\left(10^{3}\right)$ & 140 & 99 & 422 & 108 & 56 \\
Mean & 1726 & 1371 & 4730 & 874 & 752 \\
Maximum & 2 & 2 & 4 & 2 & 2 \\
Minimum & & & & & \\
& & & & & \\
Number & 27 & 25 & 58 & 30 & 22 \\
Mean & 137 & 79 & 554 & 105 & 110 \\
Maximum & 5 & 5 & 7 & 5 & 3 \\
Minimum & 0.58 & 0.59 & 0.84 & 0.72 & 0.72 \\
Vol, Number) & & & & & \\
\hline
\end{tabular}

Turnover denotes the mean hourly turnover in Dutch guilders, Volume denotes the mean hourly number of shares traded, Number denotes the mean number of official price changes and Cor(vol, Number) denotes the correlation between hourly trading volume and hourly price changes.

Therefore we adjusted our data for systematic intra-daily effects following the procedure of Gallant, Rossi and Tauchen (1992). In particular, we adjust for socalled "hour-of-the-day"-effects (including the overnight period) using dummy variables for each period ${ }^{5}$. In addition, we remove the autocorrelation of the first order by including an AR(1) component in the dummy regression for price changes. In the adjustment process the systematic effects were first removed from the mean and subsequently from the variance of both the return and the trading volume series. The adjustment was necessary as trading volume had a autocorrelation function with peaks every seven lags because of the fact that trading volume today is heavily correlated with trading volume yesterday in the same trading hour (the $U$-curve). Because the sample period was relatively short (153 trading days) we did not correct for systematic daily effects as in Gallant, Rossi and Tauchen (1992).

In Table 6.3 and 6.4 we present summary statistics for the adjusted return and trading volume series. The adjusted return series in this table feature considerable skewness and kurtosis. In contrast to daily return series, see Chapter 5, there seems to be no serial correlation in intra-daily returns, especially after correcting for the presence of heteroskedasticity by using the Diebold Statistic.

5 See Chapter 5 for a description of the adjustment procedure. 
Table 6.2 Mean Returns, Mean Wolatility and Mean Wolumes per Trading Period

\begin{tabular}{|c|c|c|c|c|c|}
\hline & ABN-AMRO & $\mathbb{I N G}$ & Philips & Royal Outch & Unilever \\
\hline \multicolumn{6}{|l|}{ Returns } \\
\hline Period 1 & -0.028 & 0.052 & 0.124 & 0.058 & 0.035 \\
\hline Period 2 & -0.0115 & 0.014 & 0.004 & -0.008 & -0.022 \\
\hline Period 3 & 0.005 & .0 .003 & -0.009 & -0.002 & -0.017 \\
\hline Period 4 & 0.026 & 0.004 & .0 .038 & 0.002 & 0.002 \\
\hline Period 5 & -0.010 & 0.021 & -0.019 & 0.014 & 0.002 \\
\hline Period 6 & -0.022 & -0.002 & -0.043 & 0.000 & 0.004 \\
\hline Period 7 & 0.153 & 0.056 & 0.072 & .0 .032 & -0.010 \\
\hline \multicolumn{6}{|l|}{ Volatility } \\
\hline Period 1 & 0.248 & 0.212 & 1.775 & 0.417 & 0.264 \\
\hline Periad 2 & 0.122 & 0.064 & 0.187 & 0.038 & 0.043 \\
\hline Period 3 & 0.045 & 0.050 & 0.212 & 0.023 & 0.030 \\
\hline Period 4 & 0.049 & 0.043 & 0.256 & 0.018 & 0.024 \\
\hline Period 5 & 0.055 & 0.042 & 0.211 & 0.020 & 0.027 \\
\hline Period 6 & 0.048 & 0.046 & 0.264 & 0.037 & 0.029 \\
\hline Periad 7 & 0.111 & 0.110 & 0.450 & 0.073 & 0.074 \\
\hline \multicolumn{6}{|l|}{ Volume } \\
\hline Period 1 & 129192 & 102103 & 520014 & 124587 & 65024 \\
\hline Periad 2 & 151322 & 102644 & 379061 & 106654 & 57507 \\
\hline Periad 3 & 139027 & 94018 & 351834 & 96015 & 50720 \\
\hline Periad 4 & 110681 & 701118 & 279001 & 72953 & 40852 \\
\hline Period 5 & 104105 & 72949 & 338677 & 72516 & 39601 \\
\hline Period 6 & 133938 & 106882 & 435110 & 102363 & 48929 \\
\hline Period 7 & 211099 & 146965 & 653408 & 179096 & 87360 \\
\hline
\end{tabular}

Returns are multiplied with 100. Volatility measures are based on these scaled returns. Period $p$ denotes the $p$-th trading hour. The overnight period is incorporated in the first trading period, see Appendix $A$ for further details.

Furthermore intra-daily returns do not seem to be autocorrelated when aggregated over 60 minutes. The normality hypothesis can be rejected for the volume series too. Trading volume is heavily (serially) autocorrelated as well. Our objective is to investigate whether stock return volatility and trading volume can be modelled in an intra-daily fashion the same way as in the previous chapter, where Stochastic Volatility and GARCH models were estimated. The Granger Causality tests are able to give us a brief overview of the dynamics between trading volume and volatility during the trading day. Table 6.5 presents F-statistics of Granger Causality tests for returns and volume and volatility and volume respectively. Several conclusions can be drawn from these tables: first, there is almost no causality (in the Granger sense) between returns and volume. Volatility does however seem to cause trading volume in some cases (ABN-AMRO, ING and Philips), whereas trading volume also has an impact on volatility in three cases (Philips, Royal Dutch and Unilever). The Philips stock is an exception here again: there is a very turbulent relationship in both directions. The main result of these tests is that the dynamic relationship can not easily be captured and that is uncertain which direction(s) it has. 
Table 6.3 Summary Statistics Adjusted Hourly Returns:

\begin{tabular}{|c|c|c|c|c|c|}
\hline & ABN-AMRO & $\mathbb{N N G}$ & Phillips & Royal Dutch & Unilever \\
\hline Mean & 0.016 & 0.020 & 0.013 & 0.005 & .0 .001 \\
\hline S.D. & 0.310 & 0.285 & 0.692 & 0.299 & 0.264 \\
\hline Minimum & -2.775 & -1.023 & .7 .202 & -1.658 & -1.275 \\
\hline Maximum & 1.042 & 2323 & 2.498 & 1.360 & 1.105 \\
\hline Skewness & -0.81 & 0.48 & -1.84 & -0.01 & -0.98 \\
\hline Kurtosis & 9.88 & 7.82 & 18.68 & 5.29 & 5.76 \\
\hline$A C(1)$ & -0.0199 & 0.0115 & -0.0343 & -0.0161 & -0.0486 \\
\hline$A C(2)$ & 0.0348 & 0.0253 & 0.01123 & 0.0502 & $-0,0210$ \\
\hline$A C(B)$ & 0.0043 & 0.0149 & 0.0465 & 0.0046 & 0.0119 \\
\hline$A C(4)$ & 0.0491 & -0.0026 & 0.0780 & 0.0352 & -0.0547 \\
\hline$A C(5)$ & 0.0475 & 0.0200 & 0.0450 & 0.0389 & -0.0328 \\
\hline$A C(6)$ & $0.0613^{\circ}$ & $0.0617^{\circ}$ & -0.0508 & -0.0299 & $0.0786^{*}$ \\
\hline$A C(7)$ & 0.0232 & -0.0210 & 0.0475 & -0.0262 & -0.0133 \\
\hline$A C(8)$ & 0.0369 & 0.0104 & 0.0047 & -0.0376 & -0.0046 \\
\hline$A C(9)$ & -0.0143 & 0.0430 & $-0.0836^{\circ}$ & -0.0197 & -0.0081 \\
\hline$A C(10)$ & 0.0020 & -0.0262 & -0.0152 & 0.0218 & $=0.0333$ \\
\hline ARCH(20) & 19.65 & 16.56 & $89.40^{\circ}$ & 15.58 & $76.66^{\prime \prime}$ \\
\hline$L B(25)$ & 29.15 & 30.81 & $39.33^{*}$ & 22.97 & 33.35 \\
\hline $\operatorname{LB}(50)$ & 55.62 & 51.91 & $90.18^{*}$ & 43.04 & 52.63 \\
\hline$D(25)$ & 28.91 & 29.21 & 26.76 & 22.14 & 27.31 \\
\hline$D(50)$ & 53.33 & 50.98 & 59.24 & 42.08 & 48.79 \\
\hline
\end{tabular}

AC( $)$ ) denotes the autocorrelation with $p$ lags, ARCH( $\rho)$ denotes the ARCH test for heteroskedasticity with $p$ lags, $L B(p)$ denotes the Ljung-Box statistic for serial correlation with $p$ lags. D $(p)$ denotes the Diebold statistic for serial correlation with $p$ lags.

Table 6.4 Summary Statistics Adjusted Hourly Trading Volumes

\begin{tabular}{|c|c|c|c|c|c|}
\hline & ABN-AMRO & ING & Phillips & Royal Dutch & Unilever \\
\hline Mean & 139909 & 99377 & 422393 & 107726 & 557037 \\
\hline S.D. & 122281 & 87565 & 457440 & 85996 & 51627 \\
\hline Minimum & -18459 & -19077 & -52540 & -35150 & -7733 \\
\hline Maximum & 404577 & 224405 & 4714999 & 725010 & 596179 \\
\hline Skewness & 3.03 & 3.27 & 3.95 & 1.91 & 3.03 \\
\hline Kuntosis & 24.22 & 30.20 & 27.06 & 10.26 & 21.13 \\
\hline $\operatorname{AC}(1)$ & $0.3654^{*}$ & $0.3293^{\circ}$ & $0.7023^{*}$ & $0.3711^{* *}$ & $0.3763^{\circ}$ \\
\hline$\triangle C(2)$ & $0.3004^{* *}$ & $0.2449^{*}$ & $0.6416^{*+1}$ & $0.2876^{* *}$ & $0.2541^{* *}$ \\
\hline$A C(B)$ & $0.2952^{\prime \prime}$ & $0.2482^{* 4}$ & $0.5558^{8 * *}$ & $0.2394^{* * 4}$ & $0.1909^{*}$ \\
\hline$A C(4)$ & $0.2206^{\prime \prime}$ & $0.2174^{\circ}$ & $0.5226^{\circ *}$ & $0.2508^{\circ}$ & $0.1707^{*}$ \\
\hline$A C(5)$ & $0.1768^{\prime *}$ & $0.2062^{\circ}$ & $0.4711^{4 \cdot 1}$ & $0.2529^{\circ}$ & $0.1299^{* *}$ \\
\hline$A C(6)$ & $0.1534^{\prime \prime \prime}$ & $0.1959^{\circ}$ & $0.4311^{* *}$ & $0.2820^{\circ *}$ & $0.1135^{\prime \prime}$ \\
\hline$A C(7)$ & $0.1325^{4 \prime \prime}$ & $0.1719^{\circ}$ & $0.3770^{\circ}$ & $0.2399^{\circ}$ & $0.0745^{* *}$ \\
\hline $\mathrm{AC}(\mathrm{B})$ & $0.0976^{4 * t}$ & $0.1242^{\circ 4}$ & $0.3301^{* *}$ & $0.2023^{\circ \prime}$ & $0.1061^{*}$ \\
\hline $\operatorname{AC}(9)$ & $0.1269^{\circ *}$ & $0.1729^{\circ *}$ & $0.3072^{* *}$ & $0.1761^{\prime *}$ & 0.0539 \\
\hline$A C(10)$ & $0.0832^{\circ}$ & $0.1617^{*}$ & $0.2820^{\circ}$ & $0.1557^{*}$ & 0.0600 \\
\hline $\operatorname{LE}(25)$ & $576.36^{\circ}$ & $680.29^{* *}$ & $2922.78^{\circ}$ & $806.91^{\circ}$ & $387.16^{* *}$ \\
\hline $\operatorname{LB}(50)$ & $632.86^{2 x}$ & $855.35^{*}$ & $3367.81^{\circ *}$ & 824.44 & $433.44^{* 2}$ \\
\hline$D(25)$ & $284.85^{* \alpha}$ & $454.74^{\circ *}$ & $729.17^{* *}$ & $476.24^{* m}$ & $2 \| 4.61^{*}$ \\
\hline$D(50)$ & $348.67^{*}$ & $646.83^{\prime \prime}$ & $95654^{* *}$ & $494.81^{\prime \prime}$ & $273.32^{\circ *}$ \\
\hline
\end{tabular}

$\mathrm{AC}(p)$ denotes the autocorrelation with $p$ lags, $L B(p)$ denotes the Ljung-Box statistic for serial correlation with plags, $D(p)$ denotes the Diebold statistic for serial correlation with plags. 
Table 6.5 Granger Causality Tests

\begin{tabular}{|c|c|c|c|c|c|}
\hline & ABN-AMRO & ING & Philips & Royal Dutch & Unileve \\
\hline \multicolumn{6}{|c|}{$\begin{array}{l}\text { Retums and } \\
\text { Volume }\end{array}$} \\
\hline F(1)WR & 0.03 & 0.86 & $10.50^{\circ}$ & $4.56^{\circ}$ & $4.00^{\circ}$ \\
\hline$F(1) R V$ & 1.90 & $14.57^{* *}$ & $3.95^{\circ}$ & $6.38^{\prime \prime}$ & 0.08 \\
\hline$F(2)$ R & 0.93 & 0.3 .4 & $5.21^{\circ}$ & 2.84 & 2.61 \\
\hline$F(2) R V$ & 1.09 & $8.48^{11 *}$ & $5.69^{\circ}$ & 2.37 & 0.01 \\
\hline$F(5)$ VR & 1.49 & 0.79 & $2.42^{\circ}$ & 1.18 & $2.79^{\circ}$ \\
\hline$F(5) R V$ & 1.85 & $4.93^{\prime * *}$ & $7.56^{\circ "}$ & 1.25 & 0.31 \\
\hline$F(10) V R$ & $1.96^{\circ}$ & 0.61 & $2.08^{\circ}$ & 9.16 & 9.81 \\
\hline F(10)RV & 1.70 & $3.66^{\circ *}$ & $4.07^{\circ}$ & 1.07 & 0.79 \\
\hline$F(20) \mathrm{WR}$ & 1.17 & 0.61 & $2.25^{* *}$ & 1.06 & 1.43 \\
\hline$F(20) R V$ & $2.01^{\circ}$ & $2.43^{\circ *}$ & $2.82^{* *}$ & 0.75 & 0.80 \\
\hline$F(50)$ VR & 1.36 & 0.73 & $2.06^{\circ}$ & 0.93 & 1.00 \\
\hline $\mathrm{F}(50) \mathrm{RV}$ & $1.46^{\circ}$ & $1.39^{\circ}$ & $1.74^{* *}$ & 1.04 & 0.75 \\
\hline
\end{tabular}

Volatility and

volume

\begin{tabular}{|c|c|c|c|c|c|}
\hline F(1)VR2 & 0.13 & 1.44 & $45.10^{\prime \prime *}$ & $11.23^{* *}$ & $19.13^{* *}$ \\
\hline$F(1) R 2 V$ & $9.31^{\text {mit }}$ & $5.62^{*}$ & $15.79^{\prime \prime *}$ & 3.72 & 3.08 \\
\hline$F(2) \vee R 2$ & 0.81 & 0.88 & $25.83^{* *}$ & $5.61^{* *}$ & $7.86^{* *}$ \\
\hline$F(2) R 2 V$ & $8.22^{* *}$ & $5.46^{* *}$ & $13.82^{* x}$ & 2.97 & 1.82 \\
\hline F(5) VR2 & 0.62 & 1.41 & $11.33^{* *}$ & $2.42^{*}$ & $9.38^{\circ *}$ \\
\hline$F(5) R 2 V$ & $6.02^{* *}$ & $3.30^{* *}$ & $19.78^{\circ "}$ & 1.76 & 1.64 \\
\hline F(10) VR2 & 1.01 & 1.46 & $8.40^{* *}$ & 1.61 & $5.25^{* *}$ \\
\hline$F(10) R 2 V$ & $5.05^{* *}$ & $3.91^{\mathrm{**}}$ & $9.86^{* \prime \prime}$ & 1.82 & 1.70 \\
\hline F(2ONR2 & 0.85 & 0.80 & $4.97^{* *}$ & 1.26 & $2.83^{m *}$ \\
\hline$F(20) R 2 W$ & $2.94^{* *}$ & $1.78^{*}$ & $5.56^{* \prime \prime}$ & 1.29 & $1.67^{*}$ \\
\hline F(5O)VR2 & 1.15 & 0.83 & $5.15^{\circ *}$ & 0.95 & $1.43^{\prime \prime}$ \\
\hline$F(50) R 2 W$ & 1.13 & 1.08 & $3.59^{\circ 4}$ & 1.15 & 0.90 \\
\hline
\end{tabular}

F(p)VR denotes the F statistic for the null hypothesis that volume does not Granger cause returns; $F(p) R Y$ denotes the $F$ statistic for the null hypothesis that returns do not Granger cause wolume; F(p)WR2 denotes the $F$ statistic for the null hypothesis that volume does not Granger cause volatility: F( $p) R 2 V$ denotes the $F$ statistic for the null hypothesis that volatility does not Granger cause volume. "and " denote significance at the $5 \%$ and $1 \%$ level respectively; $p$ denotes the number of lags. 
Table 6.6 Summary Statistics Adjusted Transformed Returns

\begin{tabular}{|c|c|c|c|c|c|}
\hline 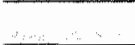 & ABN-AMRO & ING & Philips & Royal Dutch & Unilever \\
\hline Mean & -4.018 & -4.286 & -2.693 & -3.987 & -4.368 \\
\hline S.D. & 2.471 & 2.877 & 2.469 & 2.589 & 2.506 \\
\hline Minimum & -13.338 & -19.424 & -22.472 & -21.190 & -17.119 \\
\hline Maximum & 2.041 & 1.686 & 3.949 & 1.011 & 0.486 \\
\hline Skewness & 0.99 & -2.02 & -1.23 & 1.78 & -1.27 \\
\hline Kurtosis & 3.70 & 8.96 & 7.53 & 7.66 & 5.28 \\
\hline$A C(1)$ & $0.0868^{*}$ & $0.0809^{*}$ & $0.0892^{\circ}$ & 0.0348 & $0.0629^{\circ}$ \\
\hline$A C(2)$ & 0.0011 & $0.0614^{*}$ & $0.1090^{\circ}$ & -0.0008 & $0.0689^{\circ}$ \\
\hline$A C(3)$ & 0.0199 & 0.0467 & $0.1564^{* x}$ & -0.0120 & -0.0022 \\
\hline$A C(4)$ & 0.0005 & 0.0195 & $0.11417^{* *}$ & -0.0138 & -0.0086 \\
\hline$A C(5)$ & -0.0005 & -0.0221 & 0.0601 & -0.0005 & $0.0819^{\circ}$ \\
\hline$A C(6)$ & 0.0179 & 0.0148 & $0.1078^{*}$ & 0.0270 & -0.0083 \\
\hline $\mathrm{AC}(7)$ & -0.0252 & -0.0231 & $0.1136^{* 0}$ & $0.0615^{\circ}$ & 0.0038 \\
\hline$A C(B)$ & 0.0038 & 0.0342 & 0.0494 & -0.0243 & 0.0232 \\
\hline$A C(9)$ & 0.0142 & 0.0048 & $0.1089^{\circ "}$ & 0.0163 & 0.0048 \\
\hline$A C(10)$ & -0.0366 & 0.0247 & 0.0379 & -0.0059 & 0.0247 \\
\hline$\angle B(25)$ & 33.60 & 30.59 & $216.65^{\circ}$ & 24.66 & $48.57^{\prime *}$ \\
\hline $\mathrm{LB}(50)$ & 64.58 & 53.41 & $307.34^{* *}$ & 48.43 & $91.78^{\circ}$ \\
\hline$D(25)$ & 34.42 & 28.70 & $205.04^{\circ}$ & 26.44 & $47.81^{\circ *}$ \\
\hline$D(50)$ & 65.07 & 53.37 & $298.30^{\circ}$ & 50.46 & $95.10^{* *}$ \\
\hline
\end{tabular}

S.D. denotes standard deviation, $A C(p)$ denotes the autocorrelation with $p$ lags, LB(p) denotes the LjungBox statistic for serial correlation with $p$ lags, $D(p)$ denotes the Diebold statistic for serial correlation with $\rho$ lags.

\subsection{Univariate Stochastic Volatility Models}

In this section we discuss and estimate the stochastic volatility models presented in Chapter 5. It is interesting to investigate whether the degree of persistence in volatility using these models remains high for intra-daily stock return data. We estimate the intra-daily stochastic volatility models with and without incorporating the leverage effects. In Table 6.6 we present summary statistics for the transformed return series, which in general are substantially negatively skewed, implied by the log transformation and related to the "inlier" problem. If we compare the serial correlation and autocorrelation statistics of the intra-daily series with the results for daily series, we see that there is a substantial difference: except for the Philips stock returns these correlations do not have any significant values. In addition, we find partially insignificant ARCH test results in Table 6.3, which implies that we are likely to find much less persistence in the stochastic volatility models for intra-daily data?

6 Rather weak evidence of the leverage effect was found in the daily analysis so far.

* Although we expect higher frequency returns to have a larger persistence in volatility, it should be noted that the sample period is relatively short ( 153 trading days). This period is probably too short to find substantial evidence of persistence in volatility. ARCH tests for 
For reasons of comparison we first estimate the basic stochastic volatility model for adjusted stock returns without including the leverage effect and hourly trading volume. This leads to the following stochastic volatility specification, also used in Chapter 5:

$$
\begin{array}{ll}
\ln y_{t}^{2}=h_{t}+\beta_{y}+\xi_{y, t}, & \xi_{y}-N\left(0, \pi^{2} / 2\right) \\
h_{t+1}=\varphi h_{i}+\eta_{t}, & \eta_{t} \sim N\left(0, \sigma_{n}^{2}\right)
\end{array}
$$

Analogous to the analysis in the previous chapter, the distribution of the error term in the measurement equation, $\xi_{y t}$, is assumed to be normal, see Abramowitz and Stegun (1970). Consistent with our approach in Chapter 5 we estimated all stochastic volatility models on the residuals of an $\operatorname{AR}(1)$ of $y_{t}$ in order to remove any first-order correlation effects from the return series. QuasiMaximum Likelihood estimation results based on the Kalman filter for a model without trading volume and leverage are presented in Table 6.7. The results indicate that we do not find a high degree of persistence in the volatility of hourly returns. The values of $\varphi$ are extremely low for most stocks if we compare these results with those in studies for daily returns, but as expected the Philips stock is an exception ( $\varphi$ is close to one). Equations 6.1 and 6.2 do not take into account a leverage effect. Therefore we estimate the correlation parameter within the state-space formulation ${ }^{8}$. This results in the following state-space equation, see Harvey and Shephard (1994):

$$
\begin{aligned}
& \ln y_{t}^{2}=h_{t}+\beta_{y}+\xi_{y, t}, \quad \xi_{y} \sim N\left(0, \pi^{2} / 2\right) \\
& h_{t+1}=\left(\varphi-0.2242 \rho \sigma_{\eta} s_{t}\right) h_{t}+s_{t} \sigma_{\eta \eta} \rho\left(0.7979+0.2242 \ln y_{t}^{2}\right)+\eta_{t}^{*} \\
& \eta_{t}^{*}-N\left(0, \sigma_{\eta}^{2}\left(1-0.8846 \rho^{2}\right)\right)
\end{aligned}
$$

Results for the model above are presented in Table 6.8. The leverage parameter, $\rho$, is statistically significant in only one case (Philips). The other parameter estimates are not influenced by the inclusion of the leverage effect. This is con-

the daily data did not render any significant results, except for the Philips stock.

For a more detailed discussion on this model, see Chapter 5 . In addition we estimated several GARCH models, including the multiplicative specification of Chapters 4 and 5 . Results indicated that the volume exponent parameter $(\gamma)$ was statistically significant in all cases. 
sistent with the estimation results for claily stock returns in the USA, presented in the previous chapter. The next step in our empirical analysis is to include hourly trading volumes as a time-varying scale factor for conditional volatility using a model specification in a linear state space format (presented in section 5.3). We first estimate a specification without specifying the leverage parameter:

$$
\begin{aligned}
& \ln y_{t}^{2}=h_{t}+\beta_{y}+\gamma \ln V_{t}+\xi_{y, t}, \quad \xi_{y} \sim N\left(0, \pi^{2} / 2\right) \\
& h_{t+1}=\varphi h_{t}+\eta_{t}, \quad \eta_{t} \sim N\left(0, \sigma_{\eta}^{2}\right)
\end{aligned}
$$

In this specification the simple and straightforward stochastic volatility model results for $\gamma=0$. In Table 6.9 we provide parameter estimates for this model. The volume parameter, $\gamma$, is statistically significant at the $1 \%$ level in every single case. Except for the Philips case, the degree of persistence in volatility however remains low. Log-Likelihood values clearly indicate that the incorporation of trading volume in the measurement equation of the model significantly improves results. In addition, we estimated equations 6.6 and 6.7 incorporating the possibility of a leverage effect. In this specification we use the same transition equation and correlation structure as in 6.4 and 6.5. Analogous to the model without including trading volume in the measurement equation, we do not find substantial evidence of the leverage effect, see Table $6.10^{\circ}$. Comparing the estimation results with the daily results in the previous chapter, it shows that there is a similar relationship between hourly trading volume and hourly conditional volatility. Including trading volume, acting as a scale factor for conditional volatility, improves the fit significantly. The persistence in volatility is low, but including trading volume in the measurement equation does not decrease the persistence too much. This is consistent with our findings in Chapter 5.

In the next section we focus on the theoretically more appealing bivariate modelling approach, mainly based on the mixture of distributions hypothesis. In the bivariate specification, price changes and trading volume are both simultaneously influenced by a latent information variable. It is interesting to see whether we can improve our empirical analysis by estimating a bivariate model for price changes and trading volume. This modelling approach was first introduced in a univariate setting by Clark (1973) and further developed by Tauchen and Pitts (1983) and Harris (1987). In their models it is assumed that the latent mixing variable is serially independent.

Likelihood-Ratio tests show that adding the correlation parameter in the specification does not really improve results. In addition, most parameters are not affected by the inclusion of the extra leverage parameter. 
Table 6.7 Stochastic Volatility Model Estimates

\begin{tabular}{llllll}
\hline & ABN-AMRO & ING & Philips & Royal Dutch & Unilever \\
\hline$\beta_{8}$ & $-4.0166^{* *}$ & $-4.2880^{\circ *}$ & $-2.7197^{* *}$ & $-3.9878^{* *}$ & $-4.3698^{* *}$ \\
$\Phi$ & $0.3798^{* *}$ & $0.2359^{* *}$ & $0.9716^{* *}$ & 0.1272 & $0.4038^{* *}$ \\
$\sigma_{n}$ & $1.0058^{* *}$ & $1.7696^{* *}$ & $0.2032^{* *}$ & $1.3176^{* *}$ & $1.0412^{\circ *}$ \\
$L_{L}^{*}$ & -2479.7 & -2625.2 & -2451.9 & -2532.4 & -2494.9 \\
\hline
\end{tabular}

For a description of the parameters see text. LL denotes the Log-Likelihood values. "denotes statistical significance at the $5 \%$ level, " denotes statistical significance at the $1 \%$ level.

Table 6.8 Stochastic Volatility Model Estimates with Leverage

\begin{tabular}{llllll}
\hline & ABN-AMRO & ING & Philips & Royal Dutch & Unilever \\
\hline$\beta_{y}$ & $-4.0134^{* *}$ & $-4.2910^{* *}$ & $-2.6576^{* *}$ & $-3.9859^{* *}$ & $-4.3697^{* *}$ \\
$\Phi$ & $0.3679^{* *}$ & $0.1876^{* *}$ & $0.9468^{* *}$ & 0.1286 & $0.4025^{*}$ \\
$\sigma_{n}$ & $1.0162^{* *}$ & $1.7974^{* *}$ & $0.3054^{* *}$ & $1.3186^{* *}$ & $1.0413^{* *}$ \\
$\rho$ & 0.0422 & -0.1498 & $-0.4529^{* *}$ & 0.0597 & $-0.0109^{*}$ \\
$L_{L}$ & -2479.6 & -2641.2 & -2450.2 & -2532.3 & -2494.9 \\
\hline
\end{tabular}

For a description of the parameters see text. LL denotes the Log-Likelihood values. "denotes statistical significance at the $5 \%$ level, " denotes statistical significance at the $1 \%$ level.

Table 6.9 Stochastic Volatility and Volume Model Estimates

\begin{tabular}{llllll}
\hline & ABN-AMRO & ING & Philips & Royal Dutch & Unilever \\
\hline$\beta_{y}$ & $-10.4444^{* *}$ & $-11.4949^{* *}$ & $-5.2674^{* *}$ & $-10.0616^{* *}$ & $-11.1899^{* *}$ \\
$\varphi$ & $0.3587^{* *}$ & $0.2054^{*}$ & $0.9893^{* *}$ & 0.0865 & $0.1911^{* *}$ \\
$\sigma_{n}$ & $0.9226^{*}$ & $1.6971^{* *}$ & $0.0561^{*}$ & $1.2217^{* *}$ & $0.9187^{* *}$ \\
$Y^{*}$ & $0.5577^{* *}$ & $0.6452^{* *}$ & $0.9391^{* *}$ & $0.5399^{* *}$ & $0.6480^{* *}$ \\
\hline$L$ & -2460.0 & -2622.0 & -2392.7 & -2511.9 & -2458.0 \\
\hline
\end{tabular}

For a description of the parameters see text. LL denotes the Log-Likelihood values. "denotes statistical significance at the $5 \%$ level, " denotes statistical significance at the $1 \%$ level.

Table 6. 10 Stochastic Volatiility and Volume Model Estimates with Leverage

\begin{tabular}{|c|c|c|c|c|c|}
\hline & ABN-AMRO & ING & Philips & Royal Dutch & Unilever \\
\hline$\beta_{y}$ & $-10.4342^{* 4 "}$ & $-11.5116^{\prime * *}$ & $-15.0551^{*}$ & $-10.1115^{\circ}$ & $-11.1880^{\circ}$ \\
\hline$\Phi$ & $0.3559^{\circ}$ & 0,1394 & $0.8174^{* *}$ & 0.0943 & $0.1917^{\circ}$ \\
\hline$O_{i 1}$ & $0.9255^{\circ}$ & $1.7300^{\circ *}$ & $0.3748^{\circ}$ & $1.2262^{* *}$ & $0.9206^{\prime \prime}$ \\
\hline$\rho$ & 0.0121 & -0.1744 & $-0.6640^{n=}$ & 0.1146 & -0.0118 \\
\hline$y$ & $0.5569^{\circ i n}$ & $0.6465^{\circ *}$ & $0.9847^{\circ}$ & $0.5445^{\circ}$ & $0.6478^{*}$ \\
\hline LL & -2460.0 & -2621.5 & -2388.7 & -2511.6 & -2458.0 \\
\hline
\end{tabular}

For a description of the parameters see text. $L L$ denotes the Log-Likelihood values. "denotes statistical significance at the $5 \%$ level, " denotes statistical significance at the $1 \%$ level.

We relax this assumption in the next section by presenting estimation results for univariate and bivariate models with both a static and a dynamic structure in the mixing variable ${ }^{10}$.

10 Taylor (1994) shows that the univariate mixture models can be regarded as a Stochastic Volatility model , see also Chapters 2 and 5 . 


\subsection{Univariate and Bivariate Mixture Models}

A basic assumption of the standard mixture model introduced by Clark (1973)", is that the daily price change on speculative markets is the sum of a random number of stochastically independent within-day price changes, see for instance Chapters 2 and 4. The standard univariate mixture model for price changes has the following specification:

$d p_{t} \mid I_{t}=N\left(\mu_{d p}, \sigma_{d p}^{2} I_{t}\right)$

$\ln I_{t} \sim N\left(\gamma, v^{2}\right)$

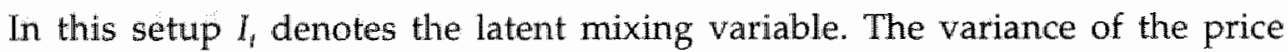
changes $\left(d p_{1}\right)$ conditional on the latent information variable is proportional to the information variable. Note that Clark (1973) assumes that the mixing variable is lognormally distributed and serially independent. In Chapter 4 we assumed that trading volume can be seen as a proxy for this information arrival process. Here we assume that price changes and trading volume are both influenced by same mixing variable. This is consistent with the approach of Tauchen and Pitts (1983) (henceforth TP83), who further developed this concept by interpreting the random mixing variable as the number of unobservable, relevant price information pieces.

In the TP83 model daily price changes and daily volumes are endogenous variables, simultaneously directed by this exogenous information arrival process, represented by $I_{i}$. In a simple sequential Walrasian framework, TP83 and Harris (1987) derive a bivariate normal distribution for both variables, conditional on the daily number of information arrivals. Here the traders' demand for a single security is a linear function of the difference between their reservation prices and the current market price. Within a trading day a series of price relevant information events takes place, which leads the traders to adjust their portfolios of stocks. Applying the equilibrium condition, the resulting change in the market clearing price is the average of all trader's' reservation price changes ${ }^{12}$. If the reservation price changes are centred around zero and independently and identically distributed with respect to all traders and all events, it is possible to show that the price change and corresponding trading volume are uncorrelated, see also Harris (1987) for this result. It follows that post event price changes and trading volumes are realizations of i.i.d. random variables for all events.

11 See Chapters 2 and 4 for a detailed discussion on the mixture of distributions hypothesis.

12 Tauchen and Pitts (1983) discuss this equilibrium in further detail. Their model implies that trading volume is only caused by the arrival of new information. 
In a daily context the total price change $d p_{t}$ and the trading volume $V_{t}$ for at given day $t$, is the sum over price changes and volume during the trading day. Each of the price changes and trading volumes are induced by an information event. Assuming a large number of intra-daily information events, TP83 show that the joint distribution of daily price changes and trading volume follows a bivariate normal distribution conditional on the daily number of information events $I_{t}$. They symbolically represent their bivariate mixture model as follows:

$$
\begin{aligned}
& d p_{t} \mid I_{t}=N\left(\mu_{d p}, \sigma_{d p}^{2} I_{t}\right) \\
& V_{t} \mid I_{t}=N\left(\mu_{v} I_{t}, \sigma_{v}^{2} I_{t}\right)
\end{aligned}
$$

with $\operatorname{Cov}\left(d p_{t}, V_{t} \mid I_{t}\right)=0$,

Here $\sigma_{d p}, \mu_{\mathrm{v}}$ and $\sigma_{\mathrm{v}}$ are positive parameters. If we now assume that the unobservable information variable $I_{t}$ is a random variable, it follows that the unconditional joint distribution of $d p_{t}$ and $V_{t}$ is a mixture of independent bivariate normals with $I_{t}$ as the mixing variable. It is important to note that we transform Clark's and Tauchen and Pitts' theory into an intra-daily context: the total price change in one trading hour is the sum of intra-hourly price changes and volumes. This shows the importance of examining a sample with heavily traded stocks as we need sufficient information arrivals per trading hour ${ }^{13}$. As shown by TP83 and Harris (1987) the empirical implications of the bivariate mixture given in 6.10 are fully consistent with a number of regularities inherent in daily price change and trading volume series in financial markets, see for instance the results in Chapter 4. Clark (1973) and TP83 assume that the information rate variable $I_{l}$ is serially independent, whereas a number of other studies in this field allow for a positive serial dependence in the mixing variable, which leads - since the dynamic properties of the mixing variable determine the dynamics of price changes and volumes - to a serial correlation in price changes ${ }^{14}$. Thus the observed persistence in the volatility of price changes may be the result of the corresponding time series behaviour of the mixing variable. In order to estimate the model in 6.10 it is necessary to specify the stochastic process which is governing the latent mixing variable $I_{t}$. Clark (1973) and TP83 explicitly assume that the latent variable is serially independent and lognormally distributed. They find that this distribution provides a better fit

13 This implies that we assume that $t$ denotes the $t$-th trading hour of a total of 1 to $T(=1071)$ trading hours in the sample period of 153 trading days.

14 See for instance Lamoureux and Lastrapes (1990a, 1994), Gallant, Hsieh and Tauchen (1991), Danielsson and Richard (1993), Andersen (1994) and Liesenfeld (1996). 
than the Poisson distribution ${ }^{15}$. In order to retain the lognormal specification and to incorporate a serially independent information arrival process we again assume the following stochastic process for $\ln \left(I_{i}\right)=h_{i}$ :

$$
h_{t}=\gamma+\varphi h_{t-1}+v \varepsilon_{t} \quad \varepsilon_{t} \sim N(0,1)
$$

As in the transition equation in SV models, $\varphi$ denotes the persistence parameter. Combining the static model in 6.10 with the dynamic properties of the mixing variable in 6.11 we eventually get the following dynamic bivariate specification for the mixture model of TP83'

$$
\begin{aligned}
& d p_{t} \mid I_{t}=N\left(\mu_{d p}, e^{h_{t}}\right) \\
& V_{t} \mid I_{t}=N\left(\mu_{v} e^{h_{t}}, \sigma_{v}^{2} e^{h_{t}}\right)
\end{aligned}
$$

Here $h_{t}$ follows the stochastic process in 6.11 which is fully compatible with the stochastic volatility models in Taylor (1994) and Mahieu and Schotman (1996). We estimate the standard model in 6.10 both assuming an independent and a dependent process for $h_{i}$.

\subsection{Estimation Results for the Mixture Models}

In Tables 6.11 through 6.14 we present estimation results for all model specifications discussed in the previous section. In Table 6.11 and 6.12 we start with the univariate mixture model for price changes as proposed by Clark (1973), see 6.8 and 6.9. In the static version $(\varphi=0)$ where the mixing variable is serially independent and lognormally distributed, the likelihood is made up of the product of $T$ one dimensional integrals. This can be evaluated by a quadrature rule. Hence it is possible to apply maximum likelihood (ML) estimation directly here $^{17}$. In the dynamic version $(\varphi \neq 0)$ the model is however estimated with simulated maximum likelihood estimation (SML), see Danielsson and Richard

15 The choice of the appropriate distribution is also discussed in the study of Richardson and Smith (1994).

16 As in Andersen (1996) and Liesenfeld (1996) the variance of the price process is set equal to one in order to normalize the model. The bivariate mixture models are invariant with respect to an arbitrary transformation of scale in $I_{i}$.

We used a 20 point GAUSS-Hermite quadrature to compute the integrals. 
(1993), Danielsson (1994) and Liesenfeld (1996), using a simulation sample size of $\mathrm{N}=500$ and 5 iterations for the AGIS algorithm described in Appendix $B^{16}$.

The ML estimation results of the static model (Clark) for price changes are consistent with the findings in earlier empirical studies for daily data by for instance Tauchen and Pitts (1983), Danielsson (1994a) and Liesenfeld (1996). The variance parameter, $v$, is statistically significant in every case with values ranging from 0.65 (Royal Dutch) to 1.14 (Philips). The implied estimates of the excess kurtosis: $\kappa_{d p}=3\left(\exp \left\{v^{2}\right\}-1\right)$, which is a monotonically increasing function of the variance parameter ( $v$ ) of the mixing variable, are significantly greater than zero for all stocks. In some cases (e.g. Unillever) the implied excess kurtosis is close to the excess kurtosis of the sample counterparts in Table 6.3, however in other cases (Philips) it is much smaller. Hence one can conclude that the observed excess kurtosis in the distributions of price changes can only partially be explained by a lognormal mixture of normal distributions.

In order to test the robustness of the SML results of the dynamic univariate model (Clark) for price changes, we provide the estimated Monte Carlo (MC) sampling standard deviations of the maximized log-likelihoods (LL). These standard deviations are calculated by estimating the likelihood for the given SML estimates of the parameters a hundred times. Subsequently, we derive the standard deviation of the logarithm of these likelihood estimates ${ }^{19}$. The value of the $\mathrm{MC}$ standard deviations show that a simulation sample of $\mathrm{N}=500$ and 5 AGIS iterations seems to be reasonably precise. Consistent with the QML estimated stochastic volatility model in section 6.3 , see Table 6.8 , we find a low persistence represented by $\varphi$. Philips is however the obvious exception: $\varphi=$ $0.88^{20}$. If we compare the log-likelihood values of the static and the dynamic model it shows that values improve significantly in most cases. This result is confirmed by the Akaike criterion (AIC) and the Schwarz criterion (SIC), which are all lower in the dynamic case. In the dynamic case we observe that a mixture of lognormal distributions is only able to explain part of the implied excess kurtosis values. The implied excess kurtosis is measured here as follows: $\kappa_{\text {dip }}=$ $3\left(\exp \left\{v^{2} /\left(1-\varphi^{2}\right)\right\}-1\right)$.

18 The appropriate AGIS algorithm is written in GAUSS and the maximization of the logarithm of the AGIS estimate for the likelihood is done with the GAUSS application Optmum.

19 Another way of testing the robustness of the procedure is to estimate the static model with SML and compare results to ML estimates.

2i0 The same argument as before applies: in most cases the daily sample period is too short to find persistence in volatility. 
Table 6.11 The Static Uniwariate Mixture Model (Clank)

\begin{tabular}{|c|c|c|c|c|c|}
\hline & ABN-AMRO & ING & Philips & Royal Dutch & Unillewer \\
\hline$\mu_{p j}$ & $0.0179^{\circ}$ & $0.0199^{\circ}$ & $0.0435^{\prime \prime}$ & 0.0064 & -0.0026 \\
\hline$y$ & $-2.6137^{*}$ & $2.8403^{\circ}$ & $-1.4578^{\prime \prime}$ & $-2.6443^{k \cdot n}$ & $-3.0446^{* x}$ \\
\hline v & $0.6835^{\prime \prime}$ & $0.7975^{*}$ & $1.1364^{\circ . t}$ & $0.6548^{* \ldots}$ & $0.8677^{7=}$ \\
\hline$K_{d f^{\prime}}$ & 1.79 & 2.67 & 7.91 & 1.61 & 3.37 \\
\hline Lit & -219.3 & -126.4 & -957.9 & -196.1 & -35.5 \\
\hline AIC & 4447 & 258.8 & 1921.9 & 398.1 & 77.0 \\
\hline Sic & 459.6 & 273.7 & 1936.8 & 413.1 & 91.9 \\
\hline
\end{tabular}

For a description of parameters, see text. $K_{d p p}$ denotes the implied excess kurtosis estimates; $L L$ denotes the Log-Likelihood; AVC denotes the Akaike Information Criterion; SIC denotes the Schwarz Information Criterion:

Table 6.12 The Dynamic Univariate Mixture Model (Clark)

\begin{tabular}{lccccc}
\hline & ABN-AMRO & ING & Philips & Royal Dutch & Unilever \\
\hline$\mu_{\rho}$ & $0.0171^{*}$ & $0.0177^{* *}$ & $0.0480^{* *}$ & 0.0066 & -0.0004 \\
$\gamma$ & $-1.4725^{* *}$ & $-1.1603^{* *}$ & $-0.1584^{* *}$ & $-1.3926^{* *}$ & $-1.2347^{* * *}$ \\
$\varphi$ & $0.4369^{* *}$ & $0.5876^{* *}$ & $0.8825^{* *}$ & $0.4731^{* *}$ & $0.5926^{* *}$ \\
$v$ & $0.6190^{* *}$ & $0.6009^{* *}$ & $0.46588^{* *}$ & $0.5725^{* *}$ & $0.6715^{* *}$ \\
$K_{\text {du }}$ & 1.82 & 2.21 & 5.00 & 1.58 & 3.01 \\
LL & -217.4 & -121.3 & -914.9 & -193.5 & -27.0 \\
MC (s.d.) & 0.100 & 0.145 & 0.193 & 0.091 & 0.251 \\
AIC & 4.42 .9 & 250.6 & 1837.7 & 395.0 & 61.9 \\
SIC & 462.7 & 270.5 & 1857.6 & 414.9 & 81.8 \\
\hline
\end{tabular}

For a description of parameters, see text. $\kappa_{\mathrm{d}, 0}$ denotes the implied excess kurtosis estimates; $L$ denotes the Log-Likelihood, $M C$ (s.d) denotes the Mante Carlo sampling standard deviations of the maximized $L_{n}$ : $A I C$ denotes the Akaike Information Criterion; SIC denotes the Schwarz Information Criterion.

The static bivariate model is estimated directly applying the ML estimation procedure again. Inspection of results for this specification, which is identical to the specification used by Tauchen and Pitts (1983), reveals the following facts (see Table 6.13). The estimates of the persistence parameter of the mixing variable vary from 0.66 to 0.81 . In four out of five cases the persistence parameter exhibits lower values here than in the univariate setting. In the bivariate dynamic case the persistence parameter is significantly smaller than in the univariate setting for three stocks. This is consistent with the findings of Andersen (1996) and Liesenfeld (1996) who also report a decrease of the persistence parameter when trading volume is included endogenously in the model. It is quite interesting to notice that although the persistence in volatility is relatively low in most cases, values for $\varphi$ tend to decrease considerably compared to the univariate model. The estimates of the variance persistence of the mixing variable are similar to the values reported in the univariate setting, ranging from 0.5536 to 0.6762 . Finally, we can see that the Monte Carlo standard deviations are significantly smaller than in the univariate setting, indicating a very high precision of the SML results. 
Table 6.13 The Static Bivariate Mixture Model (Tauchen and Pitts)

\begin{tabular}{lccccc}
\hline & ABN-AMRO & ING & Philips & Royal Dutch & Unilever \\
\hline$H_{p}$ & 0.0107 & 0.0125 & 0.025 & 0.0064 & -0.0049 \\
$\mu_{v}$ & $1.4125^{* *}$ & $1.1948^{* *}$ & $1.1740^{* *}$ & $1.1793^{* *}$ & $0.8460^{* *}$ \\
$\sigma_{q}$ & $0.1354^{* *}$ & $-0.1010^{* *}$ & $0.1905^{* *}$ & $0.1288^{* *}$ & $0.0671^{* *}$ \\
$y$ & $-2.556^{* *}$ & $-2.7462^{* *}$ & $-1.3456^{* *}$ & $-2.6112^{* *}$ & $-3.0302^{* *}$ \\
$y$ & $0.6678^{* *}$ & $0.7101^{* *}$ & $0.8075^{* *}$ & $0.6593^{* *}$ & $0.7152^{* *}$ \\
$K_{d j}$ & 1.69 & 1.97 & 2.76 & 1.63 & 2.00 \\
$\mathrm{LL}$ & 922.3 & 1379.5 & -901.1 & 1178.7 & 2088.5 \\
AIC & -1834.5 & -2748.9 & 1812.3 & -2347.4 & -4166.9 \\
SIC & -1809.7 & -2724.0 & 1837.1 & -2322.6 & -4142.1 \\
\hline
\end{tabular}

For a description of parameters, see text. $\kappa_{\text {dit }}$ denotes the implied excess kurtosis estimates: $L$ denotes the Log-Likelihood; AIC denotes the Akaike Information Criterion; SIC denotes the Schwarz Information Griterion.

Table 6.14 The Dynamic Bivariate Mixture Model (Tauchen and Pitts)

\begin{tabular}{|c|c|c|c|c|c|}
\hline & ABN-AMRO & $\mathbb{I N G}$ & Phillps & Royal Dutch & Unilever \\
\hline$\mu_{\infty}$ & 0.0101 & $0.0109^{\circ}$ & 0.0274 & 0.0072 & 0.0053 \\
\hline$\mu_{v}$ & $1.3745^{*}$ & $1.1752^{\circ *}$ & $1.1557^{* m}$ & $1.1629^{*}$ & $0.8347^{* \ldots}$ \\
\hline $\mathrm{o}_{\mathrm{w}}$ & $0.1295^{* \ldots}$ & 0.0956 & $0.1821^{* *}$ & $0.1290^{\circ}$ & $0.0655^{* *}$ \\
\hline$Y$ & $-1.2881^{4 . " t}$ & $-1.5454^{* *}$ & $-0.3935^{m+1}$ & $-1.1721^{\circ *}$ & $-1.5906^{* *}$ \\
\hline$\varphi$ & $0.4908^{*}$ & $0.4354^{-4}$ & $0.7063^{*}$ & $0.5493^{\circ}$ & $0.4701^{* *}$ \\
\hline$v$ & $0.6019^{10 *}$ & $0.6538^{*}$ & $0.5533^{* *}$ & $0.5582^{* * 1}$ & $0.6764^{\circ .}$ \\
\hline$K_{\mathrm{dp}}$ & 1.84 & 2.08 & 2.52 & 1.69 & 2.39 \\
\hline $\mathrm{LL}$ & 1004.1 & 1452.5 & -655.8 & 1266.3 & 2177.1 \\
\hline$M C$ (s.d.) & 0.026 & 0.035 & 0.026 & 0.031 & 0.027 \\
\hline AlC & -19966 & -2893.1 & 1323.7 & -2520.4 & -4342.3 \\
\hline$S I C$ & -1966.7 & -2863.2 & 1353.5 & -2490.6 & -4312.4 \\
\hline
\end{tabular}

For a description of parameters, see text. $\kappa_{\text {dot }}$ denotes the implied excess kurtosis estimates; $L$ denotes the Log-Likelihood, MC (s.d.) denotes the Monte Carlo sampling standard deviations of the maximized LL; AVC denotes the Akaike information Criterion; SIC denotes the Schwarz Information Criterion.

Finally, comparing Log-Likelihood (LL) values of the univariate models for price changes, we can conclude that the $L L$ values increase significantly in all cases. The information criteria of Akaike (AIC) and Schwarz (SIC) confirm this result. As expected, comparing LL values for the bivariate models yields the same result.

\subsection{Interpretation of Results and Concluding Comments}

In this chapter we started with the investigation of intra-daily equity return and volume data for five heavily traded Dutch stocks. It shows that these series have the same systematic features as in most financial markets. Hourly turnover and shares traded tend to be relatively high in the opening and closing hours of the trading day, but relatively low in the hours between. This phenomenon is called the $U$-curve in the literature. This pattern can also be observed in the 
squared returns series. In order to test various empirical models in this chapter we first had to adjust the return and trading volume series for these systematic effects.

One of the purposes was to estimate the univariate stochastic volatility models presented in Chapter 5 using intra-daily data. QML estimation results for these models, with and without incorporating a leverage effect, showed that the persistence of volatility in these intra-daily series was extremely low for most stocks in the sample. The ARCH test results in the summary statistics for intradaily returns indicated this allready. A larger sample period and size can give us more evidence in the near future. A leverage effect was not found. Including trading volume as a time-varying scale factor in the measurement equation, analogous to our approach in Chapter 5, significantly improved Log-Likelihood (LL) values. The trading volume parameter was significant in all cases. Again, a significant leverage effect was not found in this specification.

In the second part of this chapter we broadened the discussion by introducing the intuitively more appealing bivariate specification for price changes and trading volumes. First however, serving as a natural benchmark, we estimated the univariate specification proposed by Clark (1973) and compared it to the bivariate specification proposed by Tauchen and Pitts (1983). These studies assumed that the unobservable mixing variable was serially independent. We extended their research by assuming a serially dependent mixing variable. This however implied that we could not use Maximum Likelihood estimation directly. Therefore we applied Simulated Maximum Likelihood estimation for the dynamic mixture models. The low persistence in the volatility of intra-daily returns was found in these estimation procedure too. Measured in Log-Likelihood values, the dynamic bivariate models seemed to outperform the static models considerably. As in Andersen (1996) we find that the persistence in volatility drops considerably when estimating a bivariate specification.

In Chapter 7 we will further develop the concept of a bivariate specification for price changes and trading volume. We will present results for a sample of daily returns and vollumes in order to be able to draw conclusions based on a large sample period. We will follow the approach of Andersen (1996) who proposes a modified mixture model based on a combination of the Mixture of Distribution Hypothesis and a market microstructure model of Glosten and Milgrom (1985). In this model Andersen (1996) is able to combine the important features of the market microstructure literature with the mixture models developed by Clark (1973), Tauchen and Pitts (1983) and Harris $(1986,1987)$. This leads to a framework with the theoretically interesting concept of the existence of so-called 
liquidity traders in financial markets. This results in a Poisson distribution for daily volume in the empirical model.

\section{Appendix A Construction of the Data Set}

Beursdata B.V., a daughter of the Amsterdam Stock Exchange was kind enough to provide us with the raw data of the 25 stocks comprising the AEX-index. For reasons of liquidity we decided to focus on a few, heavily traded, individual stocks: ABN-AMRO, ING, Philips, Royal Dutch and Unilever. The raw data file consisted of price data and (cumulative) volume data. It furthermore provided us with a time stamp for every price change and displayed some information about ex-dividend dates and stock splits. The prices and corresponding trading volumes were obtained from the Limit Order Book, where prices were officially announced stock prices and trading volumes were the number of shares traded on these prices. In our construction procedure we corrected for dividend payments, stock splits did not occur in this sample period.

The stock prices in the raw data files had to be aggregated on an hourly basis. We decided to conduct the aggregation procedure as follows: first we divided the trading day into seven periods of one hour. This implies that we had to construct a closing price for every period. In our approach this closing price was the price that was nearest in time to the period under investigation. If, for instance, we would like to have closing price for the trading period $13.30-14.30$, we selected the price that was closest to 14.30 (either earlier or later). In practice, this procedure leads to deviations of at most a few minutes. We also looked at 30-minutes and 15-minutes aggregations, but we believe that these periods are too short in time. This selection procedure leads to a data set of five stocks, spanning 153 trading days with 7 hourly periods.

\section{Appendix B Simulated Maximum Likelihood Estimation (SML)}

Due to the presence of a dynamic latent variable the normal ML can not be applied directly in the dynamic specifications. Instead we use the SML method to estimate the univariate and bivariate dynamic mixture models. This technique was developed by Danielsson and Richard (1993) and tested by for instance Danielsson (1994) and Liesenfeld (1996). Applying SML avoids the problem related to the appropriate choice of moment restrictions in the Generalized Method of Moments (GMM). Using a large number of replications SML allows to adopt the instruments of inference for ML methods. SML is expected to be more efficient than GMM as it retains the complete a priori information given by 
the structure of the model. A typical disadvantage is however a very computer intensive estimation procedure.

In order to derive the marginal distribution of the observable variables, the vector of the latent variable (here denoted: $\Lambda$ ) has to be integrated out of the trivariate joint probability function of $d P, V$ and $A$ :

$f(d P, V \mid \theta)=\int f(d P, V, A \mid, \theta) d A$

where:

B1

$d P=\left\{d p_{t}\right\}_{t=1}^{T}, \quad V=\left\{V_{t}\right\}_{t=1}^{T}$

Here $\theta$ denotes the parameter vector. The unconditional joint distribution of the observable variables $f(d P, V \mid \theta)$ is then given by an integral with a dimension determined by the number of observations (T). Danielsson and Richard (1993) propose a Monte Carlo (MC) estimation technique based on an importance sampling procedure. This allows us to estimate the integral above with a high degree of precision. Given this estimate of the integral, the log-likelihood can then be evaluated and maximized over the unknown parameter vector $\theta$. To obtain a $\mathrm{MC}$ estimate of $f(d P, V \mid \theta)$ the trivariate density is factorized in an importance sampling (ISF) function and a remainder (RF) function:

ISF: $\mu(A \mid d P, V)$

RF: $\quad h(\Lambda, d P, V)$

such that the following equation holds:

$f(d p, V, \Lambda \mid \theta)=h(\Lambda, d P, V) \mu(\Lambda \mid d P, V)$.

The importance sampling function is a density function of $A$ and therefore the integral shown above can be expressed in terms of the expectation value of $h(A$, $d P, V)$ over the distribution of $\mu(A \mid d P, V)$ :

$f(d P, V \| \theta)=E_{\mu}[h(\Lambda, d P, V)]$

Subsequently a MC estimator of the integral can be constructed by:

$f_{N}(d P, V \mid \theta)=\frac{1}{N} \sum_{n=1}^{N} h\left(A_{n^{\prime}} d P, V\right)$. 
where $\left(A_{1}, \ldots, A_{n}, \ldots, A_{N}\right)$ represents a simulated random sample of size $N$, drawn from the probability distribution $\mu(\Lambda \mid d P, V)$. Under weak assumptions, see Danielsson and Richard (1993), the estimator in B5 converges to f(dP, $V(\theta)$. The precision of the estimate is dependent on the simulation sample size $N$. A natural factorization for the standard mixture model of Tauchen and Pitts (1983), according to B3 and B4 can be obtained as follows:

$f(d P, V, \Lambda \mid \theta)=f(d P, V, \mid \Lambda) f(\Lambda)$

B6

with:

$$
f(d P, V, \mid \Lambda)=\prod_{t=1}^{T} f\left(d p_{t}, v_{t} \mid \lambda_{t}\right), \quad f(\Lambda)=\prod_{t=1}^{T} f\left(\lambda_{t} \mid \lambda_{t-1}\right)
$$

Since the variables $d p_{t}$ and $v_{1}$ conditional on the mixing variable $\lambda_{1}$, are assumed to be independent, $f\left(d p_{t}, v_{t} \mid \lambda_{t}\right)$ is given by the product of two normal distributions in the case of the bivariate mixture model. At this stage we can define the following pair of sampling and remainder functions that preserves the conditions B3 and B4:

$$
\begin{aligned}
& \mu_{0}(\Lambda \mid d P, V) \equiv \prod_{t=1}^{T} f\left(\lambda_{t} \mid \lambda_{t-1}\right) \\
& h_{0}(\Lambda, d P, V) \equiv \prod_{t=1}^{T} f\left(d p_{t}, v_{t} \mid \lambda_{t}\right) .
\end{aligned}
$$

The functions above can then be used to construct a naive estimate of the marginal distribution $f(d P, V \mid \theta)$ for a given value of the parameter vector. Therefore we draw a simulated sample from the initial remainder function and subsequently the remainder is evaluated for every $\Lambda_{0, n}$. Finally, the resulting arithmetic mean of $\left\{h_{0}\left(A_{0, n}, d P, V\right)\right\}$ across all simulations serves as a naive MC-estimate of $f(d P, V \mid \theta)$. It should be noted that a high simulation sample size is needed to achieve a reasonable degree of accuracy for the MC estimate of the integral. Therefore Danielsson and Richard (1993) propose an acceleration method called Accelerated Gaussian Importance Sampling (AGIS). This method transforms the initial remainder and importance functions in such a way that the $\mathrm{MC}$ sampling variance is minimized:

$$
\min _{\mu} \operatorname{Var}_{\mu}[h(d P, V, \mathcal{A})]
$$


with:

$$
\operatorname{Var}_{\mu}[h(d P, V, A)]=\int\left[\frac{f(d P, V, A \mid \theta)}{\mu(A \mid d P, V)}-f(d P, V \mid \theta)\right]^{2} \mu(\Lambda \mid d P, V) d A
$$

This problem unfortunately has no anallytical solution and therefore we use a numerical procedure. Let $\xi(\Lambda, Q)$ be an auxiliary reduction function. $Q$ is a matrix here, containing the parameters that have to be estimated. This function enables us to construct a new pair of remainder and importance functions in the following way:

$$
\begin{aligned}
& \mu(\Lambda \mid d P, V)=\frac{\mu_{0}(\Lambda \mid d P, V) \xi(\Lambda, Q)}{\kappa(Q)} \\
& h(\Lambda, d P, V)=\frac{h_{0}(\Lambda, d P, V) \kappa(Q)}{\xi(\Lambda, Q)}
\end{aligned}
$$

where $k(Q)$ represents the integration constant, which ensures that the new importance function is indeed a proper probability function:

$$
\kappa(Q)=\int \mu_{0}(\Lambda \mid d P, V) \xi(\Lambda, Q) d \Lambda
$$

Using the new remainder and importance functions the integral can be rewritten as:

$$
f(d P, V \mid \theta)=\int_{B^{U}} h(\Lambda, d P, V) \mu(\Lambda \mid d P, V) d \Lambda
$$

The minimizing problem above is then replaced by a search for an auxiliary function $\xi(\Lambda, Q)$, which minimizes the $M C$ sampling variance of the remainder function. The AGIS method of Danielsson and Richard (1993) restricts the search for this function to that particular class of functions, for which the new importance sampler is a $T$-dimensional multivariate normal distribution. Moreover, instead of minimizing the total MC sampling variance of the remainder function itself, the variances of the individual components of the logarithm of the remainder are minimized, for each time period separately. This leads to an auxiliary function, which is a product over $T$ exponential quadratic functions. In order to determine the $(3 \times 3)$ matrix $Q$, we apply an iterative estimation procedure with $k$ steps. Starting with the initial sampler $\mu_{0}$, an initial simulated sam- 
ple is drawn. The sampler is then used to run the following auxiliary regression for every time period $t=1, \ldots, T$ :

$$
\ln h_{0}=a_{1, t}+b_{1, t} \lambda_{0, n, t}+c_{1, t} \lambda_{0, n, t}^{2}+\varepsilon_{t} \quad n=1, \ldots, N
$$

The resulting OLS estimates of $a_{1, t}, b_{1, k}$ and $c_{t, t}$ can be used to construct an estimated matrix:

$$
\hat{Q}_{1, t}=\left(\begin{array}{ccc}
-2 \hat{c}_{1, t} & 0 & -\hat{b}_{1, t} \\
0 & 0 & 0 \\
-\hat{b}_{1, t} & 0 & -2 \hat{c}_{1, t}
\end{array}\right) \quad t=1, \ldots, T
$$

This procedure is repeated for $k$ steps. Danielsson and Richard (1993) show that convergence is reached fast, typically after less than 5 iterations. The simulated sample from $\mu_{k}(A \mid d P, V)$ is used to calculate the $k$ th step AGIS estimate of the integral:

$$
\hat{f}_{N}(d P, V / \theta)=\frac{1}{N} \sum_{n=1}^{N} \frac{h_{0}\left(\Lambda_{k, n^{\prime}} d P, V\right) \kappa\left(\hat{Q}_{k}\right)}{\xi\left(\Lambda_{k, n^{\prime}} \hat{Q}_{k}\right)},
$$

Finally, to obtain the SML estimate of $\theta$, this k-th step AGIS estimate of the marginal distribution is maximized with respect to $\theta$. In Appendix $C$ we describe the AGIS sampling technique in greater detail.

\section{Appendix C The Accelerated Gaussian Importance Sampler (AGIS)}

Step (0): The initial Sampler

1. Simulate a set of $N$ independent random vector $\left\{U_{1}, U_{2}, \ldots, U_{N}\right\}$, each of which is drawn from a $T$ dimensional standardized multivariate normal distribution. These $(T \times N)$ simulated random variables are used in all steps of the AGIS algorithm and constitute the so called common random numbers.

2. Use these common random numbers to generate a first set of $\left\{\Lambda_{0,1}, A_{0,2}, \ldots\right.$ $\left.A_{0, N}\right\}$ according to the initial sampling function given in $B 8$, where the value of $\lambda_{t}$ in time period $t=0$ is set equal to zero. 
3.The initial sampling function is the product of conditional univariate normal densities:

$$
\mu_{0}(\Lambda \mid d P, V)=\prod_{t=1}^{T} \mu_{0, t}\left(\lambda_{t} \mid \lambda_{t-1}\right) \equiv \prod_{t=1}^{T} f\left(\lambda_{t} \mid \lambda_{t-1}\right)
$$

Now define the following vectors:

$$
\eta_{t}=\left(\begin{array}{c}
\lambda_{t} \\
\lambda_{t-1} \\
1
\end{array}\right), \quad z_{t}=\left(\begin{array}{c}
\lambda_{t-1} \\
1
\end{array}\right)
$$

The individual components of the initial sampler are then written as:

$$
\mu_{0, t}\left(\lambda_{t} \mid \lambda_{t-1}\right)=c_{0, t}\left(z_{t}\right) \exp \left(\frac{-\eta_{t} M_{0, t} \eta_{t}^{\prime}}{2}\right)
$$

where

$$
\begin{aligned}
& M_{0, t}=\left(\begin{array}{cc}
H_{0, t} & H_{0, t} B_{0, t} \\
B_{0, t}^{\prime} H_{0, t} & D_{0, t}+B_{0, t}^{\prime} H_{0, t} B_{0, t}
\end{array}\right), \\
& c_{0, t}\left(z_{t}\right)=\frac{1}{\sqrt{2 \pi}} \sqrt{H_{0, t}} \exp \left(\frac{z_{t} D_{0, t} z_{t}^{\prime}}{2}\right)
\end{aligned}
$$

with $E_{0}\left(\lambda_{1} \mid \lambda_{t-1}\right)=-B_{0, t} z_{1}$ and $\operatorname{Var}_{0}\left(\lambda_{t} \mid \lambda_{t-1}\right)=\left(H_{0, t}\right)^{-1}$ as the sequential conditional means and variances of the initial sampler characterized by the parameters given in equation 6.11 , the following matrices are identified:

$$
M_{0, t}=\frac{1}{v^{2}}\left(\begin{array}{ccc}
1 & -\varphi & -\gamma \\
-\varphi & \varphi^{2} & \varphi \gamma \\
-\gamma & \varphi \gamma & \gamma^{2}
\end{array}\right), \quad D_{0, t}=\left(\begin{array}{ll}
0 & 0 \\
0 & 0
\end{array}\right),
$$




$$
B_{0, t}=\left(-\varphi_{1}-\gamma\right) \quad H_{0, t}=\frac{1}{\nu^{2}}
$$

Step (k): $\quad k$-th step AGIS Sampler and k-th step AGIS estimate.

1. Use the set of simulated random vectors of the previous step $k-1:\left\{\Lambda_{k-1, n}\right\}$ to run the auxiliary regressions described in the introduction of appendix $B$ and calculate the matrices:

$\hat{Q_{k}}=\hat{Q}_{k, t}$

for $t=1$ to $T$ accordingly.

2. Then we proceed in the same fashion as for the initial sampler for the $k$-th step. Here the sequential conditional moments of the $k$-th step are:

$E_{k}\left(\lambda_{t} \mid \lambda_{t-1}\right)=-B_{k, t} z_{t}$ and $\operatorname{Var}_{k}\left(\lambda_{t} \mid \lambda_{t-1}\right)=\left(H_{k, t}\right)^{-1}$.

Defining the selection matrix:

$S_{t}=\left(\begin{array}{lll}1 & 0 & 0 \\ 0 & 0 & 1\end{array}\right), t=2, \ldots . ., T+1$,

the matrices $\left\{M_{k, 1}, M_{k, 2} \ldots, M_{k, T}\right\}$, are calculated by backward recursion, beginning in period $t=T$ and ending in period $t=1$ :

$$
M_{k, t}=M_{0, t}+Q_{k, t}+S_{t+1}^{\prime} P_{k, t+1} S_{t+1}, \quad t=1, \ldots, T,
$$

where:

$$
P_{k, t+1}=\left\{\begin{array}{cl}
D_{k_{k} t+1}-D_{0, t+1} & \text { if } t=1, \ldots, T-1 \\
0 & \text { if } t=T
\end{array}\right.
$$

Once the matrices are calculated the sequential, conditional moments of the k-tht step AGIS sampler are determined and used to generate a new set of vectors $\left\{\Lambda_{k, 1}, A_{k, 2 s} \ldots A_{k, N}\right\}$.

3. The $k$-th step AGIS estimate of $f(d p, V \mid \theta)$ is calculated according to equation B16 by: 
$f_{N, k}(d P, V \mid \theta)=\frac{1}{N} \sum_{n=1}^{N} \frac{h_{0}\left(\Lambda_{k, n^{\prime}} d P, V\right) \kappa\left(\hat{Q}_{k}\right)}{\xi\left(\Lambda_{k, n}, \hat{Q}_{k}\right)}$

where the integration constant is given with:

$$
\kappa\left(\hat{Q}_{k}\right)=\left(\prod_{t=1}^{T} \sqrt{\frac{H_{0, t}}{H_{k, t}}}\right) \exp \left(-\frac{P_{k_{0}, 1}}{2}\right) .
$$

The work of Danielsson and Richard (1993) shows that the application of the AGIS algorithm reduces the necessary sample size dramatically. 


\section{CHAPTER 7}

\section{A Bayesian Analysis of Stock Return Volatility and Trading Volume}

\subsection{Introduction}

The relationship between stock return volatility and trading volume has been a subject on the research agenda for a few decades now. The results of these studies can be used in various fields of financial economics, but its main applications are used in the dynamic portfolio allocation and the pricing of options. A better understanding of the underlying stochastic volatility process enables us to use the insights of modern finance in a more sophisticated way. Clark (1973) started the discussion by presenting the intuitively appealing Mixture of Distributions Hypothesis (MDH). As shown in previous chapters, the $\mathrm{MDH}$ posits that stock returns and trading volumes are jointly dependent on the same underlying, latent information flow variable. Empirical studies by Epps and Epps (1976), Tauchen and Pitts (1983) and Harris (1986, 1987) largely confirmed the predictions of this hypothesis. Recent work by, for example, Lamoureux and Lastrapes (1994), Richardson and Smith (1994) and Liesenfeld (1996) revealed some shortcomings of the standard mixture hypothesis. In a direct test of the standard mixture model, Richardson and Smith (1994) state that linking price changes and trading volume to the same latent information flow via a bivariate conditional normal distribution may not be the correct specification. Lamoureux and Lastrapes (1994) estimate the time series behaviour of the mixing variable. They conclude that it does not account fully for the observed persistence in volatility.

In the last decade a substantial number of papers in the market microstructure literature focused on the link between return volatility and trading volume. Most models in this area of research assume that price movements are caused by the arrival of new information and the process that incorporates this information into market prices. Important variables in these models are trading volume, the number of trades and liquidity. Most work is devoted to explain the intra- 
daily relationship between volatility and volume'. A first approach to merge the insights of the MDH with those of the market microstructure theory is an empirical model of the daily return-volume relationship developed by Andersen (1996). This model is explicitly motivated by the results of the market microstructure models by Glosten and Milgrom (1985), Kyle (1985) and Admati and Pfleiderer $(1988,1989)$. Andersen (1996) combines several, important features of these models - for instance an asymmetric information structure and the presence of liquidity or noise traders- with the MDH and the related concept of stochastic volatility. The resulting model, called the Modified Mixture Model (MMM), is estimated with a dynamic AR(1) stochastic volatility process for the latent rate of information arrival, as proposed by Andersen (1994).

In this chapter we start with presenting the MMM as well as the main ideas of the underlying theoretical model of Glosten and Milgrom (1985). Andersen (1996) provides a testable version of this model. Subsequently, he tests this model on the heavily liquid IBM common stock which is listed on the New York Stock Exchange (NYSE). In the estimation stage he uses the Generalized Method of Moments (GMM) technique to estimate several empirical specifications. One of the contributions of our study is that we use a different technique to estimate the empirical model. In particular, we construct an algorithm based on Markov Chain Monte Carlo (MCMC) simulation methods and Bayesian analysis. Jacquier, Polson and Rossi (1994) estimate a univariate stochastic volatility model using these techniques. We adapt and extend their ideas by estimating both a univariate model for stock returns and a bivariate model for stock returns and trading volume. The resulting bivariate specification is equivalent to the modified version of the mixture model as advocated by Andersen (1996). In order to be able to compare our results directly with Andersen's study we decided to use the same stock return and trading volume series (IBM) for our analysis. We are particularly interested whether we are able to confirm that the persistence parameter will decrease significantly in the MMM. A further major advantage of our estimation method is that it enables us to study the latent information process. This is not possible when GMM or Simulated Maximum. Likelihood methods are used.

The remainder of this chapter is organized as follows. In order to give the modified mixture model more theoretical background we first present the original model developed by Glosten and Milgrom (1985) in section 7.2. In section 7.3 we derive an empirical version of the bivariate model, which is similar to the

1 One of the few exceptions to this rule is a paper by Foster and Viswanathan (1995), who present a market microstructure model able to explain several interday dependencies: for instance an inverted $U$-curve for trading volume during the week.

2 Here we would like to thank Torben Andersen for providing us with the data set. 
specification used by Andersen (1996). Section 7.4 contains a discussion on the MCMC estimation technique in greater detail. In section 7.5 we present summary statistics and other characteristics of the IBM stock return and corresponding trading volume series. Section 7.6 provides simulation results for the univariate model, using the MCMC algorithm in the spirit of Jacquier, Polson and Rossi (1994). We present histograms of the respective draws of parameters simulating a univariate SV model. In section 7.7 we show results for the modified mixture model. We will compare our simulation results directly with those of Andersen (1996) and indirectly with those of other studies, for instance Liesenfeld (1996), who estimates a similar model setting using simulated maximum likelihood estimation (SML) and a German data base ${ }^{3}$. Finally, section 7.8 contains an interpretation of results and some concluding comments.

\subsection{The Theoretical Model}

The modified version of the $\mathrm{MDH}$ is in fact based on a theoretical model by Glosten and Milgrom (1985), henceforth GM. Their model is particularly suitable as it is explicitly structured to explain the process of information arrival and assimilation that occurs shortly after a piece of relevant information enters the market. GM base their model in an environment where there is a single market for an asset with a random liquidation value. Information on the terminal value of this asset enters into the market and all market players could possibly receive this information in a different way. At every point in time three different groups of risk-neutral traders are active in the market for this asset: a specialist, informed traders and uninformed traders. GM assume at this point that investors arrive to the market sequentially in random order. In this setting informed traders obtain private signals regarding the true value of the asset. GM show that these private information arrivals eventually induce a dynamic learning process that results in prices that fully reflect the content of the information through the sequence of trades and transactions. This period is referred to as a so-called price discovery or information assimilation phase. This turbulent period is then followed by an equilibrium phase.

The dynamics of GM's model are best characterized by a market place where each piece of new information relevant for the terminal value of the asset leads to a period of price discovery followed by a temporary equilibrium. Because of the frequent arrival of new information, all agents revise their estimates of the terminal value on a continuous basis. This information is either gathered from public signals, observable by each market player in a similar fashion, or from private sources that are only received by the traders with a significant informa-

The SML method is used in the empirical part of Chapter 6. 
tion advantage. Next to that, each trader is able to derive information from the sequerice of transaction prices.

Andersen (1996) formalizes the dynamics of the model as follows: $C_{i}$ is the common information set at time $t$ and each trader's information set is denoted by $\varphi_{i}$. From the outline above it follows that $\varphi_{i}$ consists of $C_{t}$ plus a possible private information set, dependent on the group of investors the particular trader belongs to. The value the specialist assigns to the asset is the expected value conditional on his current information: $P_{i}=E\left[V \mid S_{t}\right]$, where $V$ denotes the terminal value of the asset and $S_{t}$ denotes the information set at time $t$ of the specialist. GM assume that the specialist knows the structure of traders in the market as well as the structure of news arrivals taking place. The specialist works under a zero profit constraint. GM and Andersen (1996) explicitly state that $P_{1}$ will not be the quoted price as the specialist is able to observe whether the next agent buys or sells. This can be interpreted as an additional source of information. The specialist will quote prices of $V$ conditional on $S_{t}$ plus the additional buy or sell information in his order book. This implies that the specialist will never regret a trade ex post. Transaction prices then follow a martingale and therefore observed prices for $V$ at time $t$ are fair assessments of the future value of the asset.

Before we present the empirical version of this model in the next section we proceed analogous to Andersen (1996) by assuming that uninformed traders arrive at the market according to a constant Poisson information arrival process with intensity $m_{0}$ per day. The presence of liquidity traders is necessary in this context, see Kyle (1985), because they are able to provide the market with liquidity, thereby helping to circumvent the no-trading theorem by Milgrom and Stokey (1982). Andersen (1996) and GM subsequently assume that these liquidity traders have inelastic demand and supply functions, which implies that such a trader will buy or sell with probability one half. In contrast, informed traders base their trading decision on the expected value of the asset conditional on their information set, $\varphi_{t}$. The information sets of informed investors are of course correlated but not necessarily identical, which leaves room for initial disagreement among informed traders. An important point is however that the value they and other traders assign to the asset converges during the price discovery phase. This implies that there is a direct relationship between the informativeness of private information signals and the arrival rate of traders with an information advantage. The theory of GM is in fact meant to describe the price and information arrival process within a day. Andersen (1996) however translates their theory into a daily framework. Thereby he avoids a lot of

4 See also O'Hara (1995) for a detailed discussion on the no-trading theorem. 
complications associated with short run dependencies in informed trading. The properties of the return and volume series at the daily level are driven largely by the number of information arrivals per day, see also Chapters 5 and 6 . In the next section we show Andersen's formal derivation of an empirical and testable version of the GM model.

\subsection{The Empirical Model}

In the market place set by GM the market moves from one temporary equilibrium to the other, during and across trading days, in response to a large number of information arrivals each trading day. Using the same framework as Clark (1973) and Tauchen and Pitts (1983) and assuming a large number of daily information arrivals, Andersen (1996) presents a modified version of the mixture of distributions hypothesis. He starts with the following return specification:

$$
R_{t} \mid K_{t} \sim N\left(0, \sigma^{2} K_{t}\right)
$$

Here $R_{t}$ denotes the daily return, which is the logarithmic difference in prices of two consecutive trading days. $K_{1}$ is the intensity of information arrivals, measured relative to a benchmark of a fixed, large number of information arrivals and $\sigma^{2}$ is a constant scaling factor. This implies that returns are conditionally normal, but the variances of the returns reflect the intensity of the information. This result is similar to the more general theorem presented by Clark (1973), which is described in Chapters 2 and 4 . In this setting the dynamics of return volatility is solely dependent on the properties of the information flow in the market, see also Lamoureux and Lastrapes $(1990,1994)$ for this result. In contrast to these and other related studies, Andersen (1996) assumes that daily trading volume $\left(V_{t}\right)$ can be divided into an informed $\left(I V_{t}\right)$ and a noisy $\left(N V_{t}\right)$ component. In addition to that, he assumes that the noise trading part of trading volume is governed by a stochastic process with an arrival intensity of $m_{i}$ per day. This implies that the noise component of trading volume is directed by a time-invariant Poisson process $P o\left(m_{0}\right)$. Consequently, the systematic part of daily trading volume is due to the dynamics of the underlying process of information arrival.

Using this result and the insights of the GM model in the previous section, Andersen (1996) presents a framework in which each informed trader on average makes only a few trades per day. Combined with the expressions for noise 
and informed components of trading volume this eventually leads to the following distribution for daily trading volume:

$$
V_{t} \mid K_{i} \sim P a\left(m_{0}+m_{1} K_{i}\right)
$$

Volume is Poisson distributed, conditional on $K_{\mathrm{r}}$. In this setting $m_{0}$ reflects the noise component of trading volume, i.e. liquidity trades, and $m_{1}$ is the factor of proportionality, or the informed component of trading volume, which is proportional to the information flow. Andersen (1996) shows that this factor determines how strongly daily trading volume fluctuates in response to the arrival of unexpected news. Moreover, he shows in the formal derivation of the empirical model that $m_{1}$ consists of the product of two other factors: the maximum number of insiders that might obtain a private signal and the expected number of trades by an insider. These factors are however not identifiable and therefore we ignore these parameters. We proceed along the same path. Andersen (1996) normalizes the system by setting $\sigma$ in 7.1 to 1 , which leads to the following return equation:

$$
R_{t} \mid K_{t} \sim N\left(0, K_{t}\right)
$$

Here it becomes clear that the return volatility in 7.3 is identical to the information flow and therefore the unobserved information process $\left(K_{i}\right)$ can be interpreted as a stochastic volatility process, see Andersen (1994) and Taylor (1994). At this point we have to bear in mind that the trading volume series generally exhibits a strong time trend, especially when measured over a time period longer than a few years. Andersen (1996) shows for the IBM stock that there is indeed a trend present in the trading volume series. In order to be able to compare our results with those of Andersen (1996), we decided to apply the same detrending procedure. In this procedure the daily volume series are detrended by extracting a time trend $\left(a_{t}\right)$ from the observed volume series:

$$
V o_{t}=V_{t} a_{t}
$$

where $V_{0}$, denotes the observable volume series and $V_{t}$ the stationary volume series. It should be pointed out here that we face a scaling problem here, see Andersen (1996) and Liesenfeld (1996). The estimated time trend, $\hat{a}_{t}$ will at best reproduce the true underlying trend up to a certain proportionality factor $(c)$ :

5 The formal derivation and assumptions made are displayed in Andersen (1996), page 176. Andersen argues that the Poisson approximation is more precise than a normal approximation.

6 In the empirical section we implement the detrending procedure using a nonparametric regression with a Normal kernel. Here we would like to thank Torben Andersen for providing a copy of his GAUSS program. 
$c \hat{a_{t}}=a_{t}$

where $c$ is an unknown positive constant. The detrended volume series then becomes:

$$
\hat{V}_{t}=\frac{V o_{t}}{\hat{a}_{t}}=c V_{t}
$$

This leads to the following distribution for daily trading volume:

$$
\hat{V}_{t} \mid K_{t} \sim c \cdot P o\left(m_{0}+m_{1} K_{t}\right) \text { with } m_{0} \geq 0, \quad c>0
$$

The detrending procedure introduces a nuisance parameter $c$ that can be estimated, see also Liesenfeld (1996). The detrended volume series in 7.6 is given by a product of a Poisson distributed random variable $V_{t}$ and a positive constant $c$. The resulting empirical specification is a combination of the return 7.3 and volume 7.7 equations. The difference with the standard bivariate mixture model for price changes and trading volume, see Tauchen and Pitts (1983) and Chapter 6 , is in the specification of the volume equation. In our modified version of the mixture hypothesis there is a much stronger link with the market microstructure literature. This link is represented by assuming the presence of liquidity traders in the market, represented by the noisy part of trading volume. In addition, we assume a conditional Poisson distribution in the volume specification, instead of the conditional normal distribution. This explicitly respects the non-negativity constraint for trading volume. Note that the return specification is left unchanged. Thus, by accounting for an information independent part of trading volume, the MMM can be interpreted as a generalization of the standard bivariate Tauchen and Pitts (1983) mixture model in Chapter 6.

Because of the Poisson distribution, the density function is only defined for integer-valued random variables. This however cannot be ensured as $V_{t}$ and $c$ are real-valued scalars. Hence Liesenfeld (1996) concludes that Maximum Likelihood (ML) estimation using a Poisson distribution cannot be applied directly. If one however assumes that $\left(m_{0}+m_{1}\right)$ is large enough, the Poisson distribution can be approximated by a corresponding normal distribution:

$$
\hat{V}_{t} \mid K_{t} \sim N\left(c\left[m_{0}+m_{1} K_{t}\right], c^{2}\left[m_{0}+m_{1} K_{t}\right]\right) \text { with } m_{0} \geq 0, c>0
$$

Liesenfeld (1996) uses this volume specification in a simulated maximum likelihood procedure, originally proposed by Danielsson and Richard (1993). Andersen (1996) estimates his empirical model with the Generalized Method of Moments (GMM). We will estimate the MMM with a different estimation technique based on Bayesian analysis. To estimate the model we first need to spec- 
ify the stochastic process, which is assumed to govern the latent number of information arrivals $\left(K_{\ell}\right)$. In line with Andersen's approach we propose a full dynamic presentation for the mixing variable: Andersen (1996) makes a few remarks that are relevant in the selection of a dynamic representation of the information arrival process. Information on a company tends to be positively correlated: unexpected announcements tend to be followed by several other, often related news items. Consequently Andersen (1996) states, looking at the bulk of empirical research on volatility processes, that the dynamic process must display positive conditional dependencies. He proposes two possible specifications: a standard SARV model (see Chapter 2) and an exponential SARV specification, which is equivalent to a lognormal specification for information arrival. Bearing in mind the clustering of news arrivals, we concentrate on the lognormal Stochastic Volatility model for the latent variable. This leads to the following specification for the empirical version of the modified mixture model:

$$
\begin{aligned}
& y_{t} \mid h_{t} \sim N\left(0, \exp \left(h_{t}\right)\right), \\
& V_{t} \mid h_{t} \sim \operatorname{Po}\left(m_{0}+m_{1} \exp \left(h_{t}\right)\right), \\
& h_{t}=\mu+\varphi h_{t-1}+\eta_{t}, \quad \eta_{t} \sim N\left(0, \sigma_{\eta}^{2}\right),
\end{aligned}
$$

where we introduced $h_{t} \equiv \ln \left(K_{t}\right)$ as the logarithm of the latent information process?

\subsection{The Estimation Procedure}

In this section we will describe issues relating to the alternative estimation method for the modified mixture model $7.9-7.11$. In particular, we propose to construct an algorithm based on Markov Chain Monte Carlo (MCMC) simulation techniques. All parameters in the model are estimated using Bayesian analysis. Note that the model without a trading volume equation 7.10 is exactly equal to the univariate Stochastic Volatility (SV) model, presented in previous chapters and studied extensively in the literature, see Andersen and Sørensen (1996), Jacquier, Polson and Rossi (1994), Mahieu and Schotman (1996), Kim and Shephard (1994), and Ruiz (1994) among others. The techniques for estimating these simple SV models can be extended in order to deal with the estimation of

7 Note that our latent information arrival process differs slightly from the SARV model Andersen (1996) primarily uses. The logarithmic specification remains close to the processes that have been proposed in theoretical financial models for stochastic volatility as, for example, in Hull and White (1987). 
the parameters and the latent information process in the bivariate modified mixture model of Andersen (1996). Andersen (1996) estimates the MMM using Hansen's (1982) Generalized Method of Moments (GMM). GMM is a relatively fast and robust method for estimating dynamic latent variable models. A major advantage of GMM is that the implications of the distributional assumptions of a model can be estimated adequately. A serious disadvantage of GMM is however that no estimate of the latent process itself can be made. For financial applications, such as the pricing and hedging of options, an estimate of the volatility process is required. Another paper investigating Andersen's (1996) model is Liesenfeld (1996). Here a Simulated Maximum Likelihood (SML) estimation procedure, advocated by Danielsson and Richard (1993), is applied. This approach also suffers from the disadvantage that the latent information process itself is not estimated.

From the previous paragraph it has become clear that a need exists for an estimation method of the MMM that gives us an estimate of the latent information process and the associated volatility process. In this paper we will build on the MCMC methods of Shephard and Pitt (1995). These methods allow a simultaneous estimate of both the latent variable and the unknown parameters. In the following we will sketch our estimation procedure. More details on the algorithm can be found in the Appendix. The parameters in the MMM can be collected in the vector $\hat{v} \equiv\left(\mu, \varphi, \sigma_{\eta}^{2}, m_{0}, m_{1}\right)$. Note that we do not include the volume scaling parameter $c$ in this vector, which is used in Andersen (1996). Our setup implies that estimates for $m_{0}$ and $m_{1}$ include the volume scaling ${ }^{9}$. This leads to the observation that we cannot directly test hypotheses on these parameters, see Andersen (1996). However, we are still able to measure the fractions of the average daily volume that are independent $\left(m_{0}\right)$ and dependent $\left(m_{1}\right)$ on the information flow, respectively.

We apply a Bayesian estimation procedure for the parameters. We use both informative and non-informative priors. The constant $\mu$ in the latent variable transition equation has a non-informative prior distribution which leads to a normal posterior. For the transition parameter $\varphi$ we chose a Beta distribution prior on the interval $(-1,1)$, with a mean of 0.90 and a standard deviation of 0.10 . The resulting posterior is non-conjugate, which led us to sample from it by using an accept-reject algorithm. The prior distribution for $\sigma_{\eta}{ }^{2}$ is an inverse

8. Another problem with GMM is the possible inefficiency of the parameter estimates, resulting from choosing a particular set of moments, see Andersen and Sorensen (1995). Gallant and Tauchen (1996) fix this problem in their Efficient Method of Moment (EMM) estimation procedures. However, EMM does not provide us with an estimate of the latent variable either.

9 This implicitly means that we estimate $c m m_{\theta}$ and $c m_{1}$ respectively. 
Gamma distribution which leads to an inverse Gamma posterior. These priors are similar to the ones used in Shephard and Pitt (1995). The two parameters in the volume equation 7.10 were sampled from non-conjugate posterior distributions using accept-reject algorithms. The priors for $m_{0}$ and $m_{1}$ are a Gamma and a Normal distribution respectively. The parameters in these prior distributions for $m_{0}$ and $m_{l}$ are chosen to have an implied mean and standard deviation that correspond to the GMM parameter estimates in Andersen (1996).

The MCMC algorithm that we employ cycles through six conditional distributions. The first five correspond with the parameters $v$ and the sixth distribution corresponds with the vector of latent variables. Let $\theta_{1 i}$ be the parameter vector with the $i$-th parameter deleted. We can then draw from the distributions:

$$
f\left(\hat{v}_{i} \mid Y_{T}, V_{T}, \hat{h}_{T}, \theta_{i i}\right)
$$

with:

$$
Y_{T} \equiv\left\{y_{t}\right\}_{t=1}^{T}, V_{T} \equiv\left(V_{t}\right\}_{t=1}^{T}, h_{T} \equiv\left\{h_{t}\right\}_{t=1}^{T},
$$

where the last stage of the estimation procedure refers to the conditional distribution of the latent variables $h_{T}$ :

$$
f\left(h_{T} \mid Y_{T}, V_{T}, h_{T}, \vartheta_{1 i}\right)
$$

In each round of the MCMC algorithm a new element from the vector $\left(v, h_{T}\right)$ is drawn. Each draw replaces the old value for that element and the algorithm moves to the next element. As Tierney (1994) has shown this algorithm converges to drawing from the joint distribution of the parameters and the latent variables, under mild conditions that the densities are positive over their support. For common lengths of financial time series, draws from the conditional distributions can be prohibitive as the conditional distribution of the latent variables is proportional to a $T$-dimensional integral, see Jacquier, Polson and Rossi (1994). This is due to the nonlinear relation between the measurement equations 7.9 and 7.10 and the transition equation 7.11. Much attention has been given to this issue, as was described above. Here we follow the approach of Shephard and Pitt (1995) who propose to draw blocks of states instead of single states as in Jacquier, Polson and Rossi (1994). Within the MCMC chain the length of the blocks is determined by so-called knots that are randomly drawn from the states of the previous round of the MCMC. As opposed to single move samplers as in Jacquier, Polson and Rossi (1994), the multi move block samplers are quicker and show much less autocorrelation in successive draws from the chain. We use the pseudo Metropolis-Hastings algorithm of Tierney (1994) to evaluate each block of latent variables. Compared to the standard Metropolis Hastings sampler, see Chib and Greenberg (1995), this algorithm allows the 
blanket function to be a dominating distribution on parts of the support only. For all technical details of this sampler we refer to the appendix.

\subsection{Data Description and Summary Statistics}

This section briefly describes the general features of the IBM stock return and volume series. As stated before we use the same data set as Andersen (1996). who uses a large sample of continuously compounded daily returns, corrected for dividends and stock splits. The investigation period starts January 2nd, 1973 and ends December 23rd, 1991, spanning 4693 return observations ${ }^{10}$. The closing prices were obtained from the Standard \& Poor's Daily Stock Price Guide. The time series of returns and detrended volumes series we use for our empirical analysis are shown in Figure 7.1. Corresponding histograms for the returns and volume series are provided in Figure $7.2^{11}$. The sample return mean is very small and the corresponding variance of returns is much higher, see Table 7.1. Daily return series display the expected excess kurtosis. Excluding the volatile period of October 1987 would obviously yield a much lower kurtosis value. Skewness values are apparently dominated by the negative value in October 1987 too. Daily IBM stock returns are therefore not in line with a normal distribution. Finally, looking at the autocorrelation coefficients shows that the IBM returns series display the usual dependencies we find in higher order moments.

Table 7.1 Summary Statistics of the IBM Stock Retum and Detrended Trading Volume Series

\begin{tabular}{lcccccc}
\hline & Mean $\left(.10^{2}\right)$ & St. Dev & Maximum & Minimum & Skewness & Kurtosis \\
\hline Returns & 1.51 & 1.46 & 10.05 & -26.09 & -1.04 & 27.82 \\
Volumes & 0.99 & 0.41 & 5.93 & 0.22 & 2.10 & 13.00 \\
\hline & $\mathrm{AC}(1)$ & $\mathrm{AC}(2)$ & $\mathrm{AC}(3)$ & $\mathrm{AC}(4)$ & $\mathrm{AC}(5)$ & $\mathrm{AC}(10)$ \\
\hline Returns & -0.031 & -0.008 & 0.005 & -0.035 & 0.025 & .0 .022 \\
Volumes & 0.521 & 0.315 & 0.249 & 0.234 & 0.223 & 0.128 \\
\hline
\end{tabular}

Number of Observations $(T)$ is 4693 ; $A C(p)$ denotes the retum autocorrelation with lag $p$; The standard error of the return autocorrelations $(1 / T)^{0.5}$ is equal to 0.015 .

10 Andersen (1996) deletes all observations between December 24 th and January 1st. For reasons of comparison we follow his approach. The summary statistics are also presented. in Andersen (1996). We still decided to include the main statistics in Table 7.1 as they will be referred to in the empirical section. Andersen (1996) also presents a table with crosscorrelations between squared returns and trading volume (not detrended) for several lags.

1 Andersen (1996) also displays the autocorrelation functions up to 50 lags for returns, squared returns and absolute returns respectively. These series are clearly not i.i.d. as the appropriate confidence bands are frequently violated. 

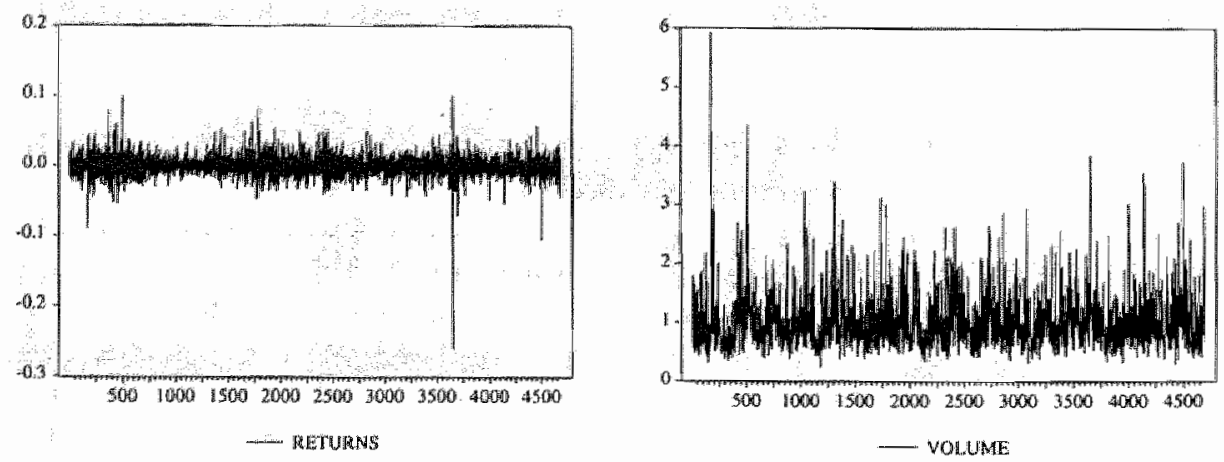

Figure 7.1 Time Series of IBM Stock Returris and Trading Volumes (1973-1991)

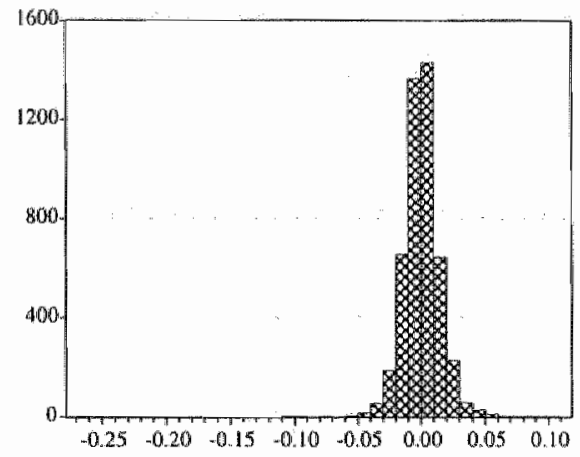

RETURNS

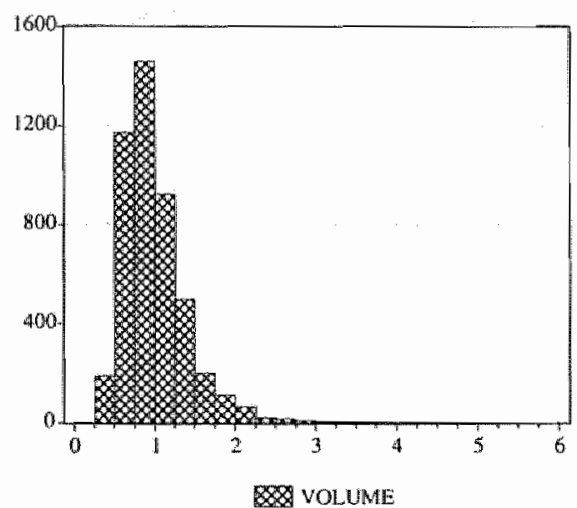

VOLUME

Figure 7.2 Histograms of IBM Stock Returns and Trading Volumes Series (1973-1991)

As noted before we have to detrend the volume series as the sample period is very long. Andersen (1996) provides evidence that there is a significant growth percentage in the IBM volume series. He describes two possible detrending procedures. We apply the first procedure where a nonparametric regression with a normal kernel is used ${ }^{12}$. In this method, the trend component that produces a nomal volume series is estimated. Subsequently, the detrended series is obtained by simply dividing each trading figure with the corresponding normal volume for that day, which leads to an average of approximately one. This

12 The other detrending method, based on a centred two year rolling sample mean, rendered the same results. We thank Torben Andersen for making his detrending algorithm available to us. 
corresponds to a two-sided moving average with weights that decline as we move further from the trading day.

Summary statistics for the detrended trading volume series are also shown in Table 7.1. The mean of this series is near unity, which is consistent with the normalization rule used in the detrending procedure. Skewness measures are clearly positive and the kurtosis value is significantly greater than three ${ }^{13}$. As expected, the lower lag autocorrelations for trading volume have very high values. In line with the standard mixture model of Harris (1987), trading volume displays a higher degree of autocorrelation than the return series ${ }^{14}$.

\subsection{Univariate Simulation Results}

The MCMC simulation algorithm, see section 7.4, is first applied to the IBM stock return series. We start estimating both the parameters and the latent information process of the underlying, univariate stochastic volatillity model without an additional measurement equation for trading volume. In Figure 7.3 histograms of the draws of each of the three parameters $\left(\mu, \varphi_{*}\right.$ and $\sigma_{\eta}{ }^{2}$ respectively) in this specification are shown together with the corresponding sequence of draws of these parameters.

For each parameter we present simulation results for 25,000 consecutive draws. Convergence of the MCMC chain is checked by comparing the draws of several sub-chains ${ }^{15}$. The marginal distributions in the histograms are in line with our expectations. Table 7.2 provides summary statistics on these distributions. The constant in the transition equation, $\mu$ (P1-Univariate), has a distribution that is severely positively skewed. Furthermore it is clear that this distribution has fat tails. The large span between minimum and maximum values for $\mu$ confirms the existence of a non-normal distribution for the draws of this parameter. The associated Monte Carlo standard error is relatively large. We can therefore conclude that the simulated values for this parameter are very unstable in the univariate model.

13 In a sub-sample analysis Andersen (1996) shows that the higher kurtosis for returns is caused by the stock market crash in 1987. In the majority of the sub-samples the kurtosis of volume is higher than for returns.

14 Andersen (1996) also reports highlly significant cross-correlations between return volatility and volume.

15 For an extensive review of current algorithms for checking MCMC output we refer to Cowles and Carlin (1996). 

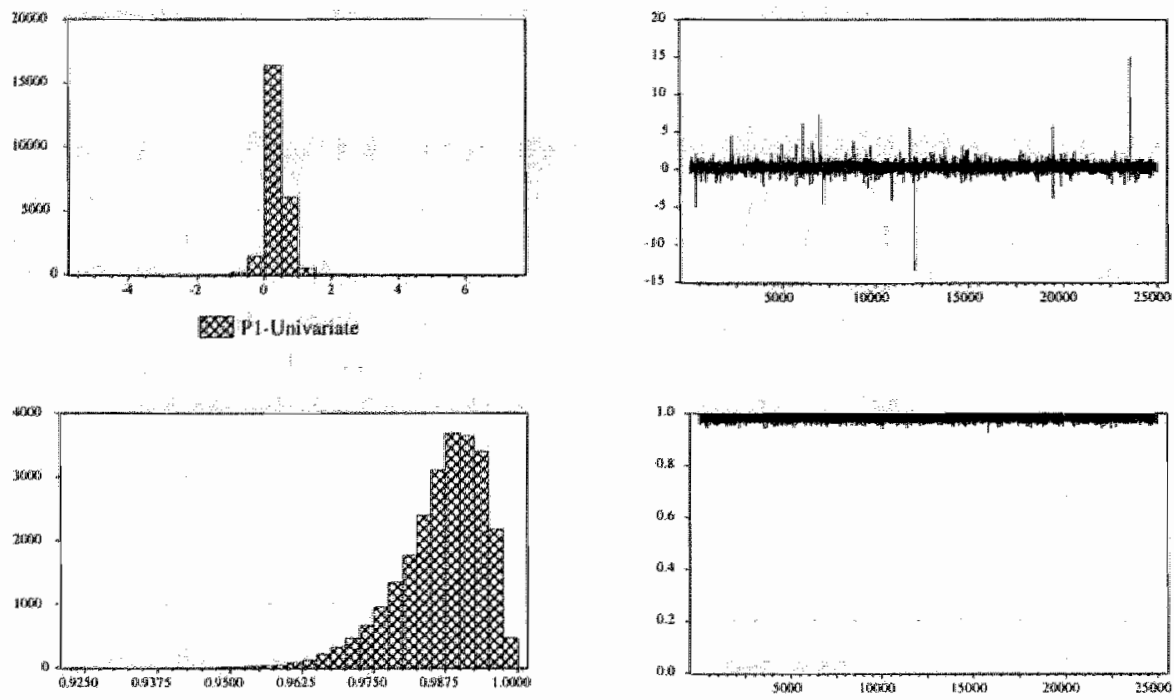

P2-Univariate
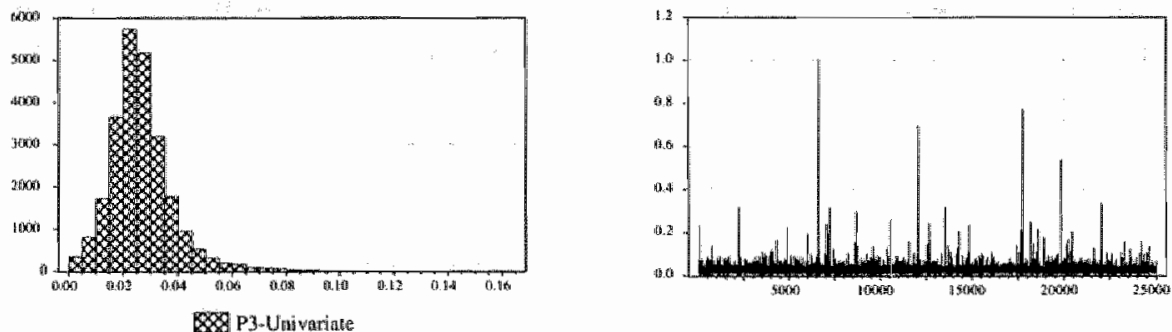

Figure 7.3 Histograms of Simulated Parameters and Sequence of Draws, Univariate SV Model

As expected, the volatility persistence parameter, $\varphi$ (P2-Univariate), is close to unity in the univariate case. The distribution of this parameter is negatively skewed with a mean of approximately 0.99 . The Monte Carlo standard errors are now very small relative to the mean value for this parameter. In addition, the sequence of draws in Figure 7.3 shows that almost every value of the persistence parameter is above 0.95. This result is consistent with Andersen (1996), who estimates a univariate SARV-model on the same series, using GMM estimation. Finally, estimates for $\sigma_{\eta}{ }^{2}$ (P3-Univariate) indicate that we have quite a lot of outliers in our simulations for this parameter too. This is also indicated by the corresponding skewness and kurtosis values and the sequence of draws in Figure 7.3. 
Table 7.2 Summary Statistics Univariate Simulation Results

\begin{tabular}{|c|c|c|c|}
\hline & $\mu$ & 9 & $\sigma_{n}^{2}$ \\
\hline Mean & 0.382 & 0.987 & 0.027 \\
\hline MC std. error & 0.359 & 0.008 & 0.018 \\
\hline Minimum & -13.499 & 0.924 & 0.000 \\
\hline Maximum & 15.078 & 0.999 & 1.006 \\
\hline Skewness & 1.140 & -1.166 & 16.631 \\
\hline Kurtosis & 230.401 & 5.084 & 679.226 \\
\hline \multicolumn{4}{|l|}{ Correlations } \\
\hline$\mu$ & 1.000 & & \\
\hline$\varphi$ & 0.013 & 1.000 & \\
\hline$\sigma_{n}^{2}$ & -0.082 & -0.105 & 1.000 \\
\hline $\mathrm{AC}(1)$ & 0.064 & 0.000 & -0.348 \\
\hline$A C(2)$ & 0.027 & -0.001 & 0.354 \\
\hline$A C(3)$ & -0.011 & 0.006 & -0.197 \\
\hline$A C(4)$ & 0.005 & -0.007 & 0.189 \\
\hline$A C(5)$ & -0.015 & 0.000 & -0.123 \\
\hline$A C(10)$ & -0.007 & 0.009 & 0.034 \\
\hline$A C(20)$ & 0.003 & -0.008 & 0.001 \\
\hline Number of Draws & 25,000 & 25,000 & 25,000 \\
\hline
\end{tabular}

MAC std error denotes the Monte Carlo standard errors of the simulated parameters, AC ( $p$ ) denotes the autocorrelation with lag $p$.

Looking at the correlations between the parameters in Table 7.2, we can conclude that these are generally low. Moreover ${ }_{s}$ autocorrelation coefficients are negligible for $\mu$ and $\varphi$. For $\sigma_{\eta}^{2}$ we find that these estimates are high for lower lags, but the coefficients decrease quickly for longer lag lengths. This result is also found in the study of Shephard and Pitt (1995). In the next section we discuss the simulation results for the bivariate, modified mixture model of Andersen (1996). In particular, we are interested whether the bivariate parameter estimates will have the same distributional properties as those in the univariate case. Another point of interest is whether we are able to confirm Andersen (1996) and Liesenfeld (1996) with respect to the decrease of the measure of persistence in vollatility $(\varphi)$.

\subsection{Bivariate Simulation Results}

The next step is to apply the MCMC simulation algorithm to the IBM return and trading volume series. We start by estimating both the parameters and the latent information process of the underlying, bivariate stochastic volatility model, which includes an additional measurement equation for trading volume. In Figure 7.4 histograms of the draws of each of the five parameters $\left(\mu, \varphi, \sigma_{r i}^{2}\right.$, $m_{0}$ and $m_{1}$ respectively) are shown together with the corresponding sequence of 
draws of these parameters. Again, we present results for 25,000 consecutive draws. A first, quick glance at the summary statistics presented in Table 7.3, tells us that the distributions of these parameters have different features when compared to the univariate case. The constant in the transition equation, $\mu$ (P1Bivariate), has a slightly lower mean, but the standard error is much lower than in the univariate setting. The displayed minimum and maximum values are in line with this phenomenon. The most interesting result is that the volatility persistence parameter, $\varphi$ (P2-Bivariate), is still close to one in the bivariate model simulations. In fact it does not even decrease in value. This is in sharp contrast with the results in Andersen (1996) and Liesenfeld (1996), who find substantially lower persistence in volatility in the case of the modified mixture model.

Andersen (1996) investigates whether the reduction of the persistence parameter could be caused by the choice of the estimation procedure. He concludes that the significant reduction in the estimated volatility persistence can not be explained by differences between estimation methods. In particular, Andersen (1996) shows that univariate GARCH and SV models for the IBM return series, estimated by both GMM and Maximum Likelihood, have a high volatility persistence $(0.99)^{16}$. The general structure of the modified mixture model could be an alternative explanation of the significant drop in persistence. Using the MCMC simulation approach we however show that the bivariate mixture model still has the same, high persistence level. This indicates that the choice of the estimation procedure may indeed be a very important factor in this issue. The mean value for $\sigma_{\eta}{ }^{2}$ does not change very much either, relative to the univariate approach, but again the Monte Carlo standard error decreases substantially in the bivariate model specification.

As mentioned before, $m_{0}$ ( $\mathrm{P} 4$-Bivariate) measures the fraction of daily trading volume independent of the underlying latent information process: $\exp \left(h_{t}\right)$. On average we find that about $82.2 \%$ of daily trading volume is unrelated to the information flow ${ }^{17}$. The fraction of daily trading volume in IBM stocks that is directly influenced by the latent information process, is measured by $m_{1}$ (P5Bivariate). The marginal distribution of $m_{1}$ has a mean of $9.2 \%$. These fractions differ from the values found by Andersen. The percentage of information-insensitive trading he finds, is much lower $(65.0 \%)$ and the information related part of trading volume is larger in Andersen's case (17.1\%).

${ }^{16}$ Note that we obtain the same result in the univariate SV model in section 7.6.

17 It should be noted that the detrended volume series has a mean of unity, which is obtained through the detrending procedure described in the previous section. 
Table 7.3 Summary Statistics Bivariate Simulation Results

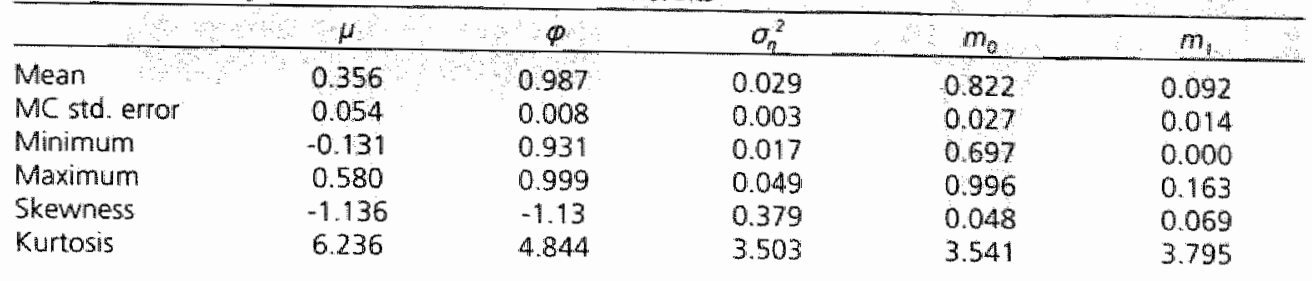

\section{Correlations}

$\begin{array}{lccccc}\mu & 1.000 & & & & \\ \varphi & -0.006 & 1.000 & & & \\ \sigma_{n}^{2} & 0.097 & -0.010 & 1.000 & & \\ m_{0} & -0.145 & -0.007 & 0.002 & 1.000 & 1.000 \\ m_{1} & -0.104 & 0.002 & 0.017 & -0.784 & 0.698 \\ A C(1) & 0.046 & 0.016 & -0.975 & 0.658 & 0.527 \\ A C(2) & 0.010 & 0.005 & 0.926 & 0.498 & 0.398 \\ A C(3) & -0.011 & -0.001 & -0.878 & 0.377 & 0.299 \\ A C(4) & 0.007 & -0.001 & 0.833 & 0.281 & 0.248 \\ A C(5) & -0.012 & -0.006 & -0.789 & 0.207 & 0.049 \\ A C(10) & -0.001 & 0.009 & 0.599 & 0.055 & -0.008 \\ A C(20) & 0.004 & 0.003 & 0.341 & -0.002 & 25,000 \\ \text { Number of Draws } & 25,000 & 25,000 & 25,000 & 25,000 & -0.000\end{array}$

MC std. error denotes the Monte Carlo standard errors of the simulated parameters, AC $(p)$ denotes the autocorrelation with lag $p$.

The correlation between the simulated bivariate parameter values, see also Table 7.3, is significant in four cases now. Particularly, $\mu$ is weakly correlated with all other parameters except $\varphi$. The strongest, negative correlation is however present between $m_{0}$ and $m_{1}: 0.784$. This may be expected because of the structure of the modified mixture model. We expect high values for $m_{1}$ whenever there is little information-insensitive trading and vice versa. In order to check whether these correlations are caused by outliers, we present scatter diagrams for these four cases. In the lower right panel of Figure 7.5 we plot the series of the two correlated volume parameters and the corresponding regression line. We can clearly see that there is a strong negative correlation between $m_{0}$ and $m_{1}$. The other diagrams are rather inconclusive. The summary statistics in Table 7.3 furthermore show that the estimated autocorrelations for the parameters of the bivariate model are still low for $\mu$ and $\varphi$. For the other three parameters we however find high coefficients for lower lag lengths. For $m_{0}$ and $m_{1}$ these values quickly decrease, but it takes more than 50 lags to obtain a low value for the autocorrelation coefficients of $\sigma_{\eta}{ }^{2}$. Possibly this problem can be solved by a multi-move block procedure for the structural parameters, proposed in Pitt and Shephard (1996). Sub-sample analysis however shows that the marginal distributions of $\sigma_{\eta}^{2}$ are very similar. 
164 Chapter 7
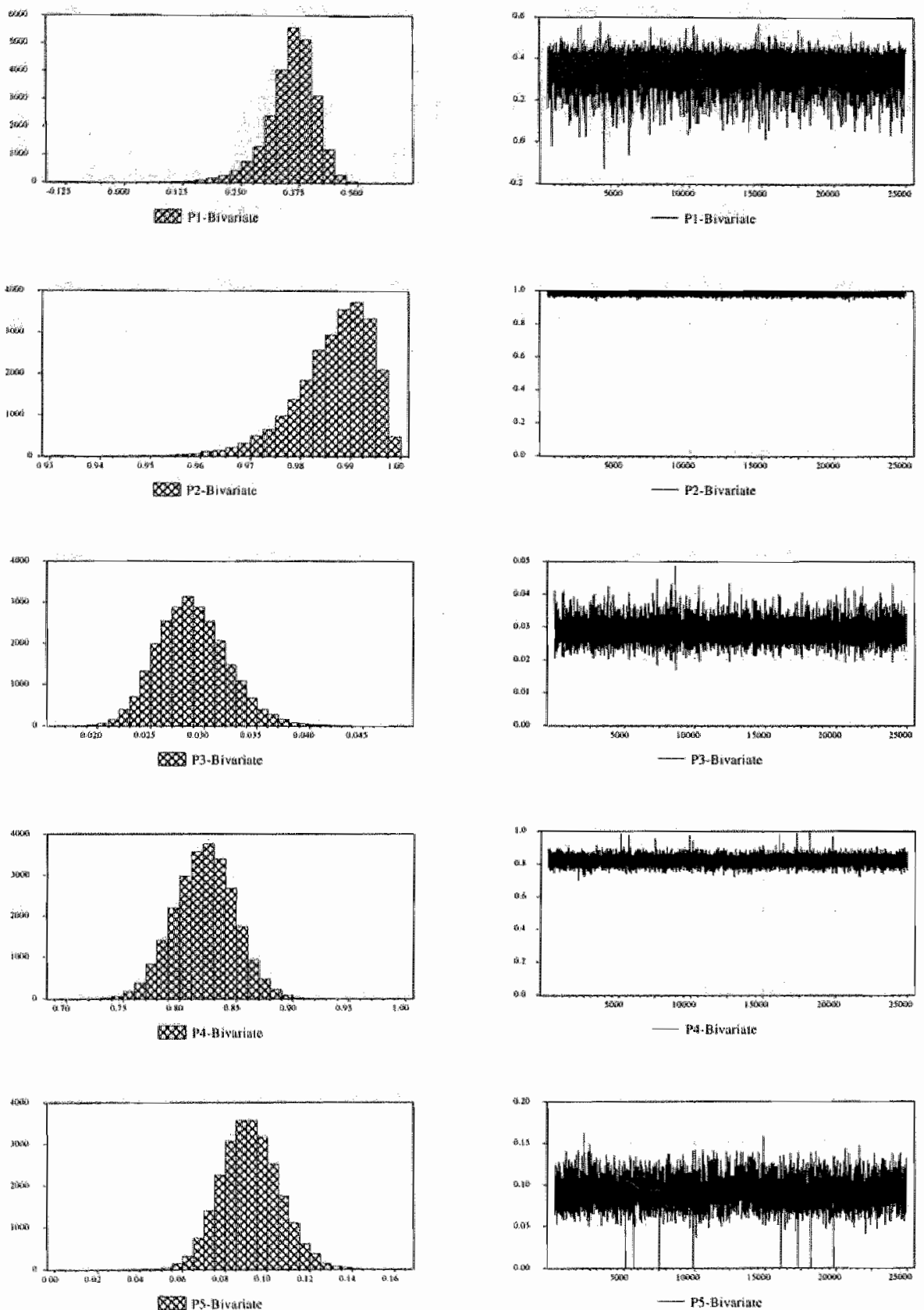

Figure 7.4 Histograms of Simulated Parameters and Sequence of Draws: Modified Mixture Model 

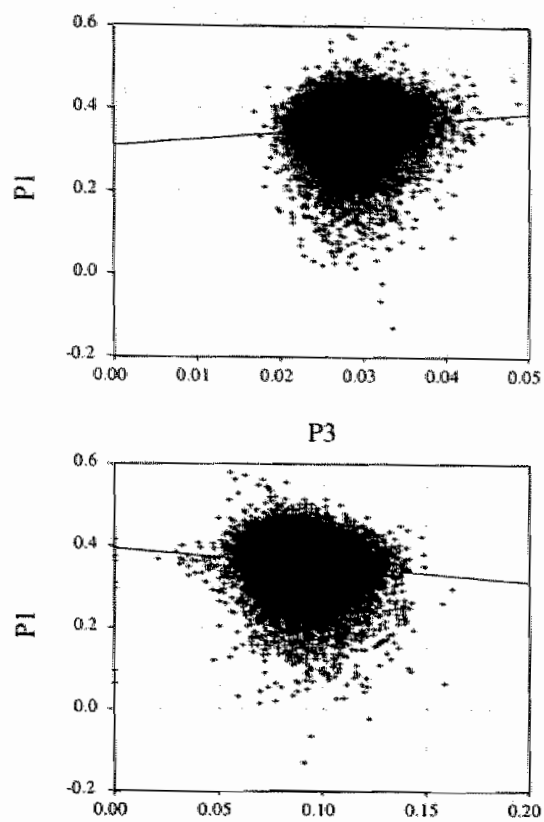

P5
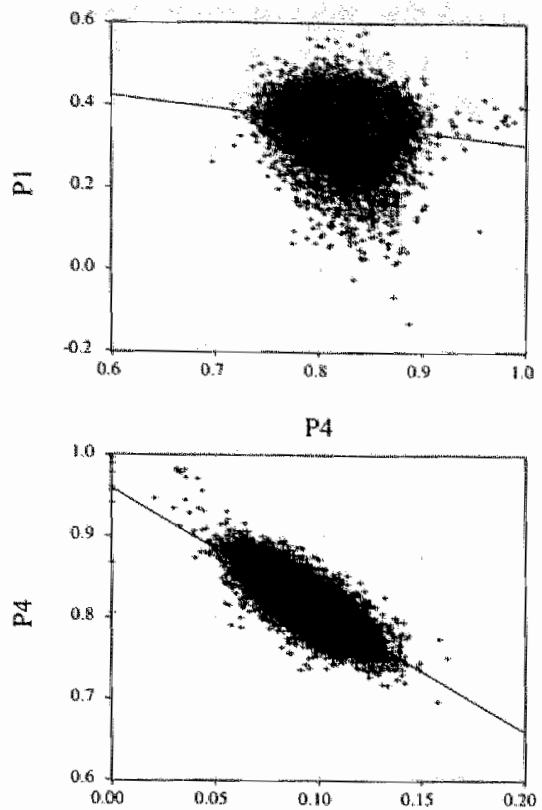

PS

Figure 7.5 Scatter Diagrams Bivariate Model

As mentioned in the introduction, one of the advantages of the MCMC simulation procedure is that it enables us to study the latent information process, which can be very useful in several areas of finance. In the upper panel of Figure 7.6 we present the estimated volatility series for the univariate model specification:

$$
\exp \left(h_{t}\right)=\frac{1}{M} \sum_{i=1}^{M} \exp \left(h_{t}^{(i)}\right), \quad t=1, \ldots, T
$$

with $h_{i}$ a time-t draw from the MCMC chain in iteration $i$. The middle panel displays the volatility series for the bivariate mixture model. From a practical point of view we are mainly interested whether we find a difference between the two estimated volatility series. Therefore we plot the difference between the two series in the lower panel of Figure 7.6. This graph shows us that these series do not differ very much in most periods, but in some volatile periods, e.g. the oil crisis in 1973 and the stock market crash of 1987, we observe differences in 
squared percentages of more than $2 \% \%^{18}$. This can have a substantial impact on the valuation of for instance derivative instruments and several strategic or tactical asset allocation topics. Summary statistics are presented in Table $7.4^{19}$. We can see that the means of both series do not differ very much.

Table 7.4 Summary Statistics Univariate and Bivariate Volatility Series

\begin{tabular}{lcc}
\hline & Univariate & Bivariate \\
\hline Mean & 1.838 & 1.823 \\
Std. Dev. & 1.340 & 1.353 \\
Minimum & 0.415 & 0.376 \\
Maximum & 22.190 & 23.607 \\
Skewness & 4.782 & 4.927 \\
Kurtosis & 48.001 & 51.573 \\
\hline
\end{tabular}

\subsection{Interpretation of Results and Concluding Comments}

In this chapter we studied the joint distribution of dailly returns and trading volumes. The contemporaneous relationship between the two variables is derived from a market microstructure model in which the presence of liquidity traders and asymmetric information structures are the main features. The resulting specification is consistent with the mixture of distributions hypothesis documented in earlier chapters. Analogous to Andersen (1996), the standard mixture model is modified by specifying a modified volume equation. In this setup trading volume is Poisson distributed, which implies that the modified mixture model explicitly accounts for the presence of liquidity traders by assuming that part of daily trading volume is unrelated to the latent information flow (noise trading) and that part is directly linked to the unobservable information process. The resulting bivariate system is governed by a random mixing variable representing the information flow or stochastic volatility variable. The lognormal stochastic volatility process is modelled as an AR(1) process. We make the following contributions to this discussion. We apply a different estimation procedure: a Markov Chain Monte Carlo based on Bayesian analysis. In contrast to the GMM approach of Andersen (1996), the MCMC method has the advantage that we are able to produce an estimate of the latent information process. This estimate can be used in several areas of modern finance.

18 It is interesting to which of the two volatility series better explains IBM option prices.

19 These results are consistent with earlier findings of Richardson and Smith (1994) who find that the information flow tends to exhibit positive skewness and large kurtosis. 


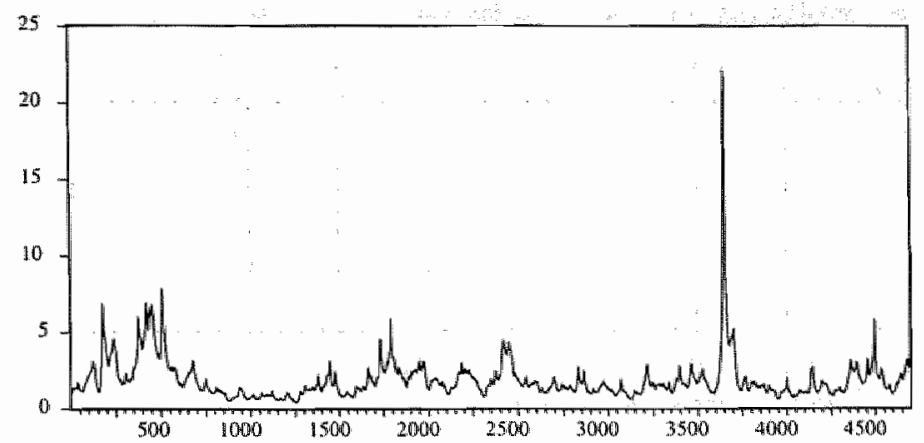

- Uniwariate States

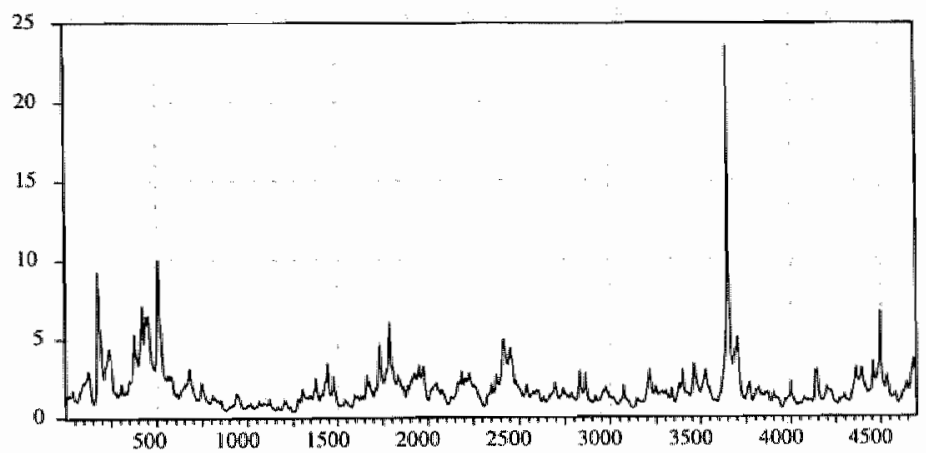

Bivariate States

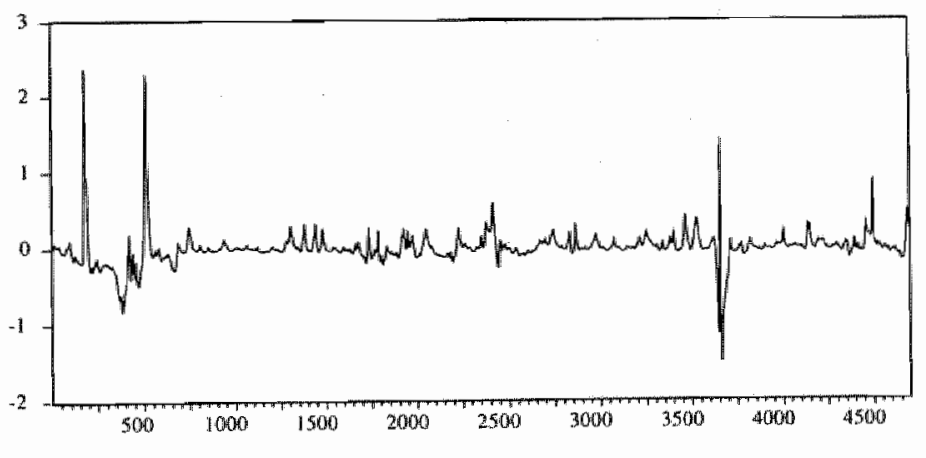

Difference in States

Figure 7,6 Estimated Volatility Series: Uniwariate Model, Bivariate Model and Difference 
The simulation results give reasons to believe that the discussion on this issue has only just started. Simulation results of the univariate stochastic volatility model confirm Andersen's result that the persistence parameter is close to unity for the liquid IBM stock return series. Monte Carlo standard errors are however rather large for the other two parameters, which indicates that the results are relatively unstable for the univariate model. Results for the bivariate mixture model are more robust in the sense that the marginal distributions of the simulated parameters are much less skewed and kurtotic. The most important result of this chapter is however that the persistence in volatility does not decrease in the bivariate model. Andersen (1996) and Liesenfeld (1996) find that the persistence in volatility drops significantly when the univariate specification is extended into a bivariate specification with trading volume. Andersen (1996) argues that this might be caused by types of information arrival processes that have a different impact on volume and return volatility persistence. News releases and periodic events as macro-economic announcements induce heavy trading volumes, but have only a short-lived effect on volatility. Failing to control for this difference could bias the estimation results. We however think that the choice of the estimation procedure also effects results. Using the same return and volume series (IBM) and a specification similar to that of Andersen (1996), we still find a high persistence in volatility in the bivariate case. Furthermore, we find for the same data that a smaller part of daily trading volume is directly related to the unobservable information process.

The modified mixture model has proven to be a very fruitful area for further research as the results clearly indicate that trading volume can be an important variable in understanding the latent information and volatility process. This, combined with our analysis in Chapter 6 , indicates that a bivariate framework may be the path to follow in new projects. Another interesting area for further research is to discriminate between various types of information that enter financial markets.

\section{Appendix A Markov Chain Monte Carlo Methods}

In this appendix we explain some technical details about our estimation technique. The basic aspects of the multi-move block sampler from Shephard and Pitt (1995) are described first. The parameters of the models are estimated by Bayesian techniques. The prior distributions and the resulting posterior distributions are presented in the second part of this appendix. 


\section{The Block Sampler}

Basically, the block sampler of Shephard and Pitt (1995) draws samples from a multivariate distribution by importance sampling. This method is applicable to a wide range of models, including the non-Gaussian state space models described in the text. One of the main advantages of this sampler is that it is considerably faster than a sampler that draws from univariate distributions sequentially. Now consider the SV model with a bivariate measurement equation that we analyse in the text:

$$
\begin{aligned}
& y_{t} \mid h_{t}-N\left(0_{r} \exp \left(h_{t}\right)\right) \\
& V_{t} \mid h_{t} \sim P 0\left(m_{0}+m_{1} \exp \left(h_{t}\right)\right) \\
& h_{t}=\mu+\varphi h_{t-1}+\eta_{t} \quad \eta_{t} \sim N\left(0, \sigma_{\eta}^{2}\right)
\end{aligned}
$$

If we now let $Y_{T} \equiv\left\{y_{1}, y_{2}, \ldots, y_{T}\right\}$ and $\left.V_{T} \equiv \mid V_{1}, V_{2}, \ldots, V_{T}\right\}$ and write down the marginal likelihood function for the parameters $\mathscr{V}$, we eventually obtain:

$$
\log f\left(Y_{T}, V_{T} ; \vartheta\right) \propto \int_{h_{1}} \cdots \int_{h_{T}} f\left(Y_{T}, V_{T} \mid H_{T} ; \vartheta\right) f\left(H_{T} ; \vartheta\right) d h_{1} d h_{T}
$$

The parameter vector $\theta$ containing the relevant hyper-parameters can only be estimated by simulation since we need to integrate out the latent variables or states $H_{T} \equiv\left\{h_{1}, h_{2}, \ldots, h_{T}\right\}$ from the likelihood function. In the special case of Gaussian models we could perform this integration analytically by the Kalman filter $^{20}$. In order to be able to perform inference on either of the parameters or the states in the SV model, we need to solve the integration problem. As analytical methods are impossible we resort to simulating the likelihood value. Suppose we can draw $M$ vectors $\left(H_{T}^{(j)}, \ldots, H_{T}^{(M)}\right)$ from the distribution $f\left(H_{\% ;}, \theta\right)$. An estimate of the integral is then:

$$
\frac{1}{M} \sum_{k=1}^{M} f\left(Y_{T}, V_{T} \mid H_{T}^{(k)} ; \mathfrak{V}\right)
$$

The number $M$ can be set in such a way that the Monte Carlo standard error is smaller than a pre-specified number, see Geweke (1994). The drawing of states from the highly dimensional density function $f\left(\mathrm{H}_{T} ; \vartheta\right)$ seems to be a daunting task at first. Recently, a number of techniques have been developed that are able to tackle this problem. One of the most investigated ways is to use simulation

20 See Harvey (1989) for an extensive overview of applications of the Kalman filter in econometrics. 
methods based on Markov Chain Monte Carlo (MCMC). The MCMC methods basically break down highly dimensional problems into smaller, more tractable problems. By combining a series of solutions to the latter we are able to construct an answer to our initial problem. In particular, for our SV model we set up a MCMC for estimating both the states and the parameters. More specifically, we use the Gibbs sampler, which cycles through a series of conditional. distributions. Tierney (1994) shows that draws from the conditional distributions converge to draws from the multivariate density. The Bayesian Gibbs sampler in our case can be represented by the following six steps:

1. Set $i=1$;

2. Get starting values for the parameters $\theta^{(i)}$ and the states $H_{T}^{(i)}$;

3. For each of the $\mathrm{P}$ parameters in $\vartheta^{(i)}$ draw a new value from the conditional distributions:

$$
f\left(\vartheta_{j}^{(i+1)} \mid Y_{T}, V_{T}, H_{T} ;\left\{q_{p}^{(i+1)}\right\}_{p=1}^{j-1},\left\{\vartheta_{p}^{(i)}\right\}_{p \neq j+1}^{p}\right) \quad j=1, \ldots, P
$$

4. Draw $H_{T}^{(i+1)}$ from $f\left(H_{T}^{(i+1)} \mid Y_{T}, V_{T}, v^{(i+1)}\right)$;

5. Set $i=i+1$;

\section{Go to 3;}

The conditional distributions of the parameters in step 3 are described later in this appendix. Here we direct our attention to drawing from the conditional distribution of the states $H_{T}$. Drawing the vector $H_{T}$ could then be split up by drawing from $T$ univariate conditional densities $f\left(h_{i} \mid Y_{T}, V_{T}, v_{,} H_{T \backslash i}\right)$ where $H_{T: i}$ is the state vector with the $i$-th state deleted. This is the approach taken by Jacquier, Polson and Rossi (1994). Shephard (1994) argues that for state space models these single-move samplers are slower with respect to multi-move samplers, in which a whole vector is sampled at once. Shephard and Pitt (1995) further refine the methods in Shephard $(1994)^{21}$.

As Shephard and Pitt (1995) note, direct sampling of the states $H_{T}$ might suffer from the fact that this distribution is highly degenerate ${ }^{22}$. Consequently, attention is directed towards sampling from the conditional distribution of the errors

21 In particular, the multi-move samplers in Shephard (1994) suffered from serial correlation between successive draws $H_{T}$.

22 For example, this might occur in an SV model with very high persistence. 
$\left\{\eta_{1}, \eta_{2}, \ldots, \eta_{T}\right\}$ in the state transition equation A1. For expositional purposes we redefine the state transition equation by:

$$
h_{t}=\varphi h_{t-1}+\sigma_{\eta} \eta_{t} \quad \eta_{t} \sim N(0,1)
$$

As the joint distribution of the errors is highly dimensional, sampling from this distribution is performed in blocks. These blocks are determined by stochastic knots, i.e. the end points change in every round of the general Gibbs sequence described above. The distribution from which we sample is then:

$\log f\left(\eta_{t-1}, \ldots, \eta_{t+k-1} \mid h_{t-1}, h_{t+k+1}, y_{t}, \ldots, y_{t+k}, V_{t}, \ldots, V_{t+k}\right)$

This conditional distribution is approximated by a multivariate Gaussian density that can be obtained from expanding $\mathrm{A} 3 \mathrm{in}$ an initial point:

$\left(\eta_{t-1}, \ldots, \eta_{t+k-1} \mid \hat{h}_{t}, \ldots, \hat{h}_{T+k}\right)$

For this reason we rewrite $\mathrm{A} 3$ as:

$\log f\left(\eta_{t-1}, \ldots, \eta_{t+k-1} \mid h_{t-1}, h_{t+k+1}, y_{t}, \ldots, y_{t+k}, V_{t}, \ldots, V_{t+k}\right) \infty$

$\log f\left(\eta_{t-1}, \ldots, \eta_{t+k-1} \mid h_{t-1}, h_{t+k+1}\right)+\log f\left(y_{t}, \ldots, y_{t+k}, V_{t}, \ldots, V_{t+k} \mid h_{t-1}, h_{t+k+1}\right)=$

$-\frac{1}{2} \sum_{j=0}^{k} \eta_{t, j-1}^{2}+\sum_{s=1}^{t+k} \log f\left(y_{s}, V_{s} \mid h_{s}\right) \approx-\frac{1}{2} \sum_{j=0}^{k} \eta_{t+j-1}^{2}+$

$\sum_{s \rightarrow t}^{t+k} \log f\left(y_{s}, V_{s} \mid \hat{h_{s}}\right)+\left(h_{s}-\hat{h_{s}}\right) \frac{\partial \log f\left(y_{s}, V_{s} \mid \hat{h_{s}}\right)}{\partial \hat{h}_{s}}+\frac{1}{2}\left(h_{s}-\hat{h_{s}}\right)^{2} \frac{\partial \log f\left(y_{s}, V_{s} \mid \hat{h_{s}}\right)}{\partial \hat{h}_{s}^{2}}$

This awkward looking density can be calculated using the Gaussian state space model with the following measurement and transition equation:

$\hat{h_{s}}-\left(\frac{\partial^{2} \log f\left(y_{s}, V_{s} \mid \hat{h}_{s}\right.}{\partial \hat{h}_{s}^{2}}\right)^{-1}\left(\frac{\partial \log f\left(y_{s}, V_{s} \mid \hat{h_{s}}\right.}{\partial \hat{h}_{s}}\right)=h_{s}+\varepsilon_{s}$,

$h_{s}=\varphi h_{s-1}+\sigma_{\eta} \eta_{t} \quad \eta_{t}-N(0,1)$

The error term $\varepsilon_{s}$ has a normal distribution with mean zero and variance:

$\operatorname{Var}\left(\varepsilon_{s}\right)=-\left(\frac{\partial^{2} \log f\left(y_{s}, V_{s} \mid \hat{h_{s}}\right.}{\partial \hat{h}_{s}^{2}}\right)^{-1}$ 
As this model is Gaussian, the simulation smoother from de Jong and Shephard (1995) can be used to draw from the required proposal density. Subsequently, draws from the smoother can be used in a Metropolis-Hastings accept-reject framework. The states around which the second order expansion is made, are obtained by iterating the moment smoother of Koopman (1993) to the mode of the density A3. Shephard and Pitt (1995) show that convergence to this mode occurs quickly.

\section{Priors and Posteriors}

In drawing the parameters from the conditional distributions we make frequent use of the pseudo dominating Metropolis-Hastings algorithm (pseudo MH) sampler, see Tierney (1994). This sampler is based on the general acceptancerejection principle, but differs in the sense that the blanket density function does not need to be dominant for the total support of the distribution we want to draw from. In the following we describe the prior and resulting posterior distributions for each of the parameters.

\section{1. $\mu$}

As in Pitt and Shephard (1996) we assume a non-informative prior distribution for $\mu$ :

$$
f(\mu) \propto \mathcal{C}
$$

The leads to the Normal posterior with the following mean and variance:

$$
E\left[\mu \mid h_{T} ; v_{\langle\mu}\right]=\frac{h_{1}+\sum_{t=2}^{T}\left(h_{t}-\varphi h_{t-1}\right)}{(1-\varphi)\left(T-1+\frac{1}{(1+\varphi)^{2}}\right)}
$$

$$
\operatorname{Var}\left[\mu \mid h_{\mathrm{T}} ; v_{\langle\mu}\right]=\frac{\sigma_{\eta y}^{2}}{(1-\varphi)^{2}\left(T-1+\frac{1}{(1+\varphi)^{2}}\right)}
$$

\section{2. $\varphi$}

For the persistence parameter in the latent process $H_{T}$ we specify a prior on $(\varphi+$ 1)/2. When we allow $-1<\varphi<1$ this assures that $0<(\varphi+1) / 2<1$. The prior 
distribution is a Beta distribution with parameters $\delta_{1}$ and $\delta_{2}$. This leads to the following posterior for the persistence parameter:

$$
\begin{aligned}
& f\left(\varphi \mid h_{T}, \sigma_{\eta}^{2}\right) \propto\left(\frac{\varphi+1}{2}\right)^{\delta_{1}-1}\left(\frac{1-\varphi}{2}\right)^{\delta_{2}-1} * \\
& \exp \left(-\frac{1}{2 \sigma_{\eta 7}^{2}}\left[\left(1-\varphi^{2}\right)\left(h_{1}-\frac{\mu}{(1+\varphi)}\right)^{2}+\sum_{t=2}^{T}\left(h_{t}-\mu(1-\varphi)-\varphi h_{t-1}\right)^{2}\right]\right)
\end{aligned}
$$

Sampling from this distribution is performed using the pseudo MH with a Gamma type blanket function.

3. $\sigma_{\eta}$

Here we use an inverse Gamma prior with parameters $S_{0}$ and $p$ as in Shephard and Pitt (1995):

$$
f\left(\sigma_{\eta}^{2} \mid \varphi\right) \propto \sigma_{\eta}^{2\left(-\frac{p}{2}+1\right)} \exp \left(-\frac{S_{0}}{2 \sigma_{\eta}^{2}}\right)
$$

This leads to the following conjugate posterior:

$$
\begin{aligned}
& f\left(\sigma_{\eta}^{2} \mid H_{T}, \varphi\right) \propto \sigma_{\eta}^{2}\left(-\frac{T+p}{2}+1\right) \\
& \exp \left(\frac{1}{2 \sigma_{\eta}^{2}}\left[S_{0}+\left(1-\varphi^{2}\right)\left(h_{1}-\frac{\mu}{1+\varphi}\right)^{2}+\sum_{t=2}^{T}\left(h_{t}-\mu(1-\varphi)-\varphi h_{t-1}\right)^{2}\right]\right)
\end{aligned}
$$

\section{4. $m_{0}$}

We specify a Gamma prior for $m_{0}$ with parameters $\gamma_{1}$ and $\gamma_{2}$. This leads to the non-conjugate posterior:

$$
f\left(m_{0} \mid V_{T}, m_{1}\right) \propto \exp \left(-\left(\gamma_{2}+T\right) m_{0} * \sum_{t=1}^{T} \ln \left[m_{0}^{\left(\gamma_{t}-1\right) / T}\left(m_{0}+m_{1} h_{t}\right)^{V_{t}}\right]\right)
$$

We sample again with the pseudo $\mathrm{MH}$ with a normal distribution as blanket. The mean and variance parameters of the blanket function were obtained by a second order Taylor expansion of the argument in the exponent. 
5. $m_{1}$

The prior for the informed component of trading volume is normal with mean $\mu$ and variance $v^{2}$. The posterior is again non-conjugate:

$$
f\left(m_{1} \mid V_{T}, m_{0}\right) \propto \frac{1}{v} \exp \left(-\frac{1}{2} \frac{\left(m_{1}-\mu\right)^{2}}{\nu^{2}}-m_{1} \sum_{i=1}^{T} h_{t}\right) \prod_{t=1}^{\mathrm{T}}\left(m_{0}+m_{1} h_{t}\right)^{V_{t}}
$$

Sampling from this distribution is performed by pseudo $\mathrm{MH}$ using a normal blanket function obtained in a similar way as for $m_{0}$. 


\section{CHAPTER 8}

\section{Concluding Comments and Suggestions for Future Research}

It is widely accepted that a better understanding of volatility leads to more pronounced and professional decision making. Large financial institutions, like for instance pension funds and insurance companies, continually face the problem how to allocate their money efficiently to the different asset classes. In recent years, stocks have become much more popular, especially in continental Europe. Institutions are shifting large, sometimes inefficient parts of fixed income positions to stock positions. In this dynamic allocation process we need to know how these asset classes behave. Volatility, as a measure of risk, is one of the important parameters for a wide range of decisions in this area. The magnitude of a bandwidth around a strategic benchmark for asset allocation, for example, should be a function of the volatility of the individual asset classes. Risk control measures as Value at Risk etc. are also based on assumptions on the volatility process of the underlying asset classes. A last example is the option theory where the return variance enters directly into derivative pricing formulas such as the Black-Scholes formula.

Until recently, most empirical work on stock return volatility was devoted to univariate time series models for which it is widely accepted that the autoregressive conditional heteroskedastic ARCH model of Engle (1982) and all its extensions have been very successful. This thesis broadens the discussion on the stock return generating process. After introducing the scope of the thesis and reviewing the existing literature in the first two chapters, we first evaluated and confirmed the existence of persistence in volatility in several stock markets in a univariate context. Using the GARCH and Stochastic Volatility methodologies we confirm high degrees of persistence in volatility. Incorporating trading volume in those univariate models led to mixed results. Although it is clear that trading volume is very important in order to understand stock market dynamics, we argued that a univariate setting is not fully in line with the theoretical underlying framework: the mixture of distributions hypothesis. Therefore we shifted our attention to a bivariate model setting in the later chapters. The issue of which estimation procedure to use was not on the agenda explicitly, but as 
we used a variety of techniques, we were able to give some comments here too. In this final chapter we will review the bulk of empirical research in this thesis.

In Chapter 3 we introduced the standard (G)ARCH methodology and a wholle variety of related univariate specifications. It is shown that the class of GARCH models imposes an autoregressive structure on the conditional variance, allowing volatility shocks to persist over time. Several conditional distributions have been used in the empirical literature. We considered alternative time series processes and distributional specifications characterizing one of Europe's main stock markets: the Frankfurt Stock Exchange. Empirical results for weekly and monthly index series clearly indicate that the student $t$-distribution is superior to the standard normal distribution. In particular, the estimated GARCH-t models appear to be reasonably successfull in accounting for both leptokurtosis and conditional heteroskedasticity from German stock return movements. The empirical results are in line with the results found for the USA, see Bollerslev e.a. (1992).

The next step was to use the insights of the Mixture of Distributions Hypothesis originally proposed by Clark (1973). This hypothesis posits a joint dependence of returns and trading activity, measured either by trading volume or the number of transactions. In Chapter 4 we interpreted trading activity as a proxy variable for the latent information process governing stock prices, following an approach of Lamoureux and Lastrapes (1990a). Inserting daily trading volume or the daily number of transactions additively into a GARCH conditional variance process, resulted in decreasing past return shocks and past conditional variances. Note that the trading activity parameters were strongly significant. This thesis contributes to the work of Lamoureux and Lastrapes (1990a) by assuming a student $t$-distribution for conditional errors and by using German individual stock returns, volumes and transactions. Subsequently, we showed that, although this univariate approach is related to the mixture hypothesis, it has several theoretical and empirical drawbacks and counter-intuitive implications. Therefore we proposed a new, multiplicative GARCH-model. We contribute to the existing literature by integrating the unobservable mixing variable out of the problem and reinterpreting trading volume as a time-varying scale factor for conditional volatility, thereby removing some of the drawbacks of the additive specification. In addition, we find that the persistence in volatility does not decrease in the multiplicative specification, whereas the additive counterpart shows a considerable drop in persistence.

In Chapter 5 we focused on several other univariate specifications where trading volume might have an important role. This resulted in a somewhat different volatility framework: Stochastic Volatility (SV). We showed that a SV framework naturally arises, when we assume that stock returns are generated by the 
mixture of distributions hypothesis. Moreover, the SV model implies that dailly trading volume can be interpreted as a time-varying scalle factor for volatility. In a univariate setting we incorporate daily trading volume in the measurement equation. Results for a sample of US stocks, previously used in a study by Lamoureux and Lastrapes (1994), clearly showed that trading volume plays an important role in stock market dynamics. In almost all cases we found a better fit for the return process using trading volume as a scale factor for volatility: Furthermore, summary statistics, sign bias tests, Granger causality tests and linear VAR estimations showed that there clearly is a strong dependency between returns, return volatility and trading activity. No conclusive evidence was found for the leverage effect, although some simple tests show that it is predominantly a tail phenomenon. Analogous to the results in Chapter 4 we should be cautious in interpreting the univariate estimation results. The findings need to be digested in further research projects and translated into reasonable hypotheses regarding the structure of the joint system. We know that the standard mixture hypothesis points to a bivariate specification: both trading volume and volatility are influenced simultaneously by the same latent flow of information. Therefore we shifted our attention to the bivariate specifications with a much stronger link to the mixture hypothesis.

Chapter 6 adds several new dimensions to the scope of this thesis. First of all, we presented results for several bivariate specifications in this chapter. Secondly, we used intra-daily data for five heavily traded Dutch stocks, all listed on the Amsterdam Stock Exchanges. Thirdly, we used a relatively new estimation technique, Simulated Maximum Likelihood estimation. The bivariate specification used in this chapter was originally proposed by Tauchen and Pitts (1983). In their study they assumed a serially independent, unobservable mixing variable. We extended their research by assuming both an independent and a dependent mixing variable. An important result of this chapter is that the empirical evidence indicates that the dynamic, bivariate mixture models are more appropriate than their static counterparts. A last remark we would like to make here is that we should be careful in handling intra-daily return and volume data. The intra-daily dynamics differs from other frequencies considerably, because all kinds of microstructural and institutional factors have an impact on the return and trading processes. This subject needs more attention and therefore it is a fruitful area for further research.

In Chapter 7 we focused on daily returns and volumes again. In particular, we proposed a bivariate specification based on a market microstructure model in which the presence of liquidity traders and asymmetric information structures are the main features. This modified mixture model was originally proposed by Andersen (1996). In this setup, part of daily trading volume is unrelated to the latent information flow (noise trading or liquidity trading) and part is directly 
linked to the unobservable information process (information trading). The resulting bivariate system is governed by a random mixing variable representing the information flow. We make several contributions to Andersen's approach. We applied a different estimation procedure, a Markov Chain Monte Carlo (MCMC) based on Bayesian analysis, whereas Andersen (1996) used the GMM approach. In our opinion; the MCMC method has the clear advantage that we are able to produce an estimate of the latent information process. This estimate can subsequently be used in several areas of modern finance, like for instance dynamic asset allocation and option pricing. The most important result in this chapter is that we found, in contrast to Andersen (1996) and Liesenfeld (1996), that the persistence in volatility did not decrease in the bivariate setting. This result has not been found before and is fully in line with the mixture hypothesis. We think that the choice of the estimation procedure possibly influences results here. In our opinion Bayesian estimation techniques should be evaluated carefully in the near future.

Throughout this thesis we have made several contributions to the literature on stock return volatility and trading volume. Especially the bivariate, modified mixture model seems to be a very interesting area for further research as the empirical results generally show that trading volume is an important variable in understanding the latent information and volatility process. It is clearly shown that a bivariate specification should be the path to follow in new research projects. Another interesting area for future research may be to discriminate between various types of information that enter financial markets. Finally, we should continue to focus on intra-daily return and volume data for this literature, as we are convinced that this will lead to a better understanding of stock market dynamics in general. 


\section{References}

ABRAMOWITZ, M. AND N.C. STEGUN (1970), Handbook of Mathematical Functions, Dover Publications, New York.

ADMATI, A.R. AND P. Pfleiderer (1988), A Theory of Intra Day Patterns: Volume and Price Variability, Review of Financial Studies, 1, 3-40.

ADMATI, A.R. AND P. PFLEIDERER (1989), Divide and Conquer: A Theory of Intra Day and Day-of-the-Week Mean Effects, Review of Financial Studies, 2, 189-223.

AKGIRAY, V. (1989), Conditional Heteroskedasticity in Time Series of Stock Returns: Evidence and Forecasts, Journal of Business, 1, 55-80.

ANDERSEN, T.G. (1994), Stochastic Autoregressive Volatility: A Framework for Volatility Modelling, Mathematical Finance, 4, 75-102.

ANDERSEN, T.G. (1996), Return Volatility and Trading Volume: An Information Flow Interpretation of Stochastic Volatility, Journal of Finance, 51 169-204.

ANDERSEN, T.G. AND B.E. SøRENSEN (1996), GMM Estimation of a Stochastic Volatility Model: a Monte Carlo Study, Journal of Business and Economics Statistics, 14, 328352.

BACKUS, D.K., A.W. GREGORY, AND ZIN S.E. (1989), Risk Premiums in the Term Structure: Evidence from Artificial Economies, Journal of Monetary Econonics, 24, 371-399.

BAILliE, R. AND T. BollersleV (1989), The Message in Daily Exchange Rates: A Conditional Variance Tale, Journal of Busimess and Economic Statistics, 7, 297-305.

BAILlie, R. AND R. DEGENNARO (1990), Stock Return and Volatility, Joumal of Financial and Quantitative Analysis, 2, 203-214.

BERA, A.K. AND C.M. JARQUE (1982), Model Specification Tests: A Simultaneous Approach, Joumal of Econometrics, 20, 59-82.

BERNDT, E.K, B. HALL, R. HALl AND J. HAUSMAN (1974), Estimation and Inference in Nonlinear Structural Models, Amnals of Economic and Sacial Measuremenl, 4, 653665.

BLACK, F. (1976), Studies of Stock Price Volatility Changes, in Proceedings of the 1976 Meetings of the American Statistical Association, Business and Economics Statistics Section, 177-181.

BLACK, F. AND M. SCHOLES (1973), The Pricing of Options and Corporate Liabilities, Journal of Political Economy, 81, 637-659.

Bollerslev, T. (1986), Generalized Autoregressive Conditional Heteroskedasticity, Journal of Econometrics, 31, 307-328. 
BoLLeRsLev, T. (1987), A Conditionally Heteroskedastic Time Series Model for Speculative Prices and Rates of Return, Review of Economics and Statistics, 69, 542547.

Bollerslev, T., R. CHOU, N. JAYARAMAN, AND K. KRONER (1992), ARCH Modeling in Finance: A Selective Review of the Theory and Empirical Evidence, With Suggestions for Future Research, Journal of Econometrics, 52, 5-59.

BOOTHE, P., AND D. GLASSMANN (1987), The Statistical Distributions of Exchange Rates: Empirical Evidence and Economic Implications, Journal of International Economics, 22, 153-167.

BRENNER, R.J., R.H. HARJES, AND K. KRONER (1994), Another Look at Alternative Models of the Short-Term Interest Rate, Unpublished Manuscript, University of Arizona.

BREUSCH, T.S., AND A.R. PAGAN (1979), A Simple Test for Heteroskedasticity and Random Coefficient Variation, Econometrica, 47, 1287-1294.

CAMPBELL, J.Y., S.J. GROSSMAN AND J. WANG (1993), Trading Volume and Serial Correlation in Stock Returns, Quarterly Journal of Economics, 101, 905-939.

CAMPBELL, J.Y. AND L. HENTSCHEL (1992), No News is Good News: An Asymmetric Model of Changing Volatility in Stock Returns, Joumal of Financial Economics, 31, 281-318.

ChEsney, M. AND M. SCOTT (1989), Pricing European Currency Options: A Comparison of the Modified Black-Scholes Model and a Random Variance Model, Journal of Financial and Quantitative Analysis, 24, 267-284.

CHIB, S. AND E. GREENBERG (1995), Understanding the Metropolis-Hastings Algorithm, American Statistician, 49, 327-335.

CHOU, R.Y. (1988), Volatility Persistence and Stock Valuations: Some Empirical Evidence using GARCH, Journal of Applied Econometrics, 3, 279-294.

CLARK, P.K. (1973), A Subordinated Stochastic Process Model with Finite Variance for Speculative Prices, Econometrica, 41, 135-156.

COHEN, K., S. MAIER, R. SCHWARTZ AND D. WhITCOMB D. (1981), Transaction Costs, Order Placement Strategy, and Existence of the Bid-Ask Spread, Journal of Political Economy, 89, 287-305.

COPELAND, T. E. (1976), A Model of Asset Trading under the Assumption of Sequential Information Arrival, fournal of Finance, 31, 1149-1168.

COPELAND, T.E. AND D. GALAI (1983), Information Effects and the Bid-Ask Spread, Jourmal of Finance, 38, 1457-1469.

COWLES, M.K. AND B.P CARLIN (1996), Markov Chain Monte Carlo Convergence Diagnostics: a Comparative Review, Journal of the American Statistical Association, 91, 883-904.

Crouch, R.L. (1970), A Non-Linear Test of the Random-Walk Hypothesis, American Economic Review, 60, 199-202.

DANIELSSON, J. (1994), Stochastic Volatility in Asset Prices Estimation with Simulated Maximum Likelihood, Journal of Econometrics, 64, 375-400.

DANIELSSON, J. AND J-F. RICHARD (1993), Accelerated Gaussian Importance Sampler with Application to Dynamic Latent Variable Models, Journal of Applied Econometrics, 8, 153-173.

DEMSETZ, H. (1968), The Cost of Transacting, Quarterly Journal of Economics, 82, 33-53. 
DIEBOLD, F.X. (1986), Comment on the Modelling in Persistence in Conditional Variance, Econometric Reviews, 5, 51-56.

DiEBOLD, F.X. (1988), Empirical Modelling of Exchange Rate Dynamics, Springer Verlag. New York.

DAEBOLD $r$ F.X. AND M. NERLOVE (1989), The Dynamics of Exchange Rate Volatility: A

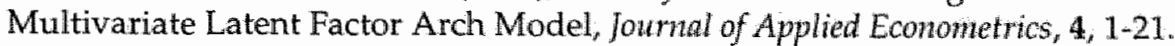

DoMOWITZ, I., AND C.S. HAKKIO (1985), Conditional Variance and the Risk Premium in the Foreign Exchange Market, Joumal of International Economics, 19, 47-66.

DROST, F.C. AND T.E. NIMAN (1993), Temporal aggregation of GARCH processes, Econometrica, 61(4), 909-927.

ENGLE, R.F. (1982), Autoregressive Conditional Heteroskedasticity with Estimates of the Variance of U.K.K. Inflation, Econometrica, 50, 987-1008.

EASLEY, D. AND M. O'HARA (1987), Price, Trade Size and Information in Securities Markets, Joumal of Financial Economics, 19, 69-90.

ENGle, R.F. AND T. BOLlerslev (1986), Modelling the Persistence of Conditional Variances, Econometric reviews, 5, 1-50.

ENGLE, R.F., D.F. HENDRY AND J.F. RICHARD (1983), Exogeneity, Econometrica, 51, 277304.

ENGLE, R.F. AND G.G.J. LEE (1993), A Permanent and Transitory Component Model of Stock Return Volatility, Unpublished Manuscript, University of California, San Diego.

ENGLE, R.F., D.M. LILIEN AND R.P. ROBBINS (1987), Estimating Time-Varying Risk Premia in the Term Structure: The ARCH-M Model, Econometrica, 55, 391-407.

ENGLE, R.F. AND V.K. NG (1993), Measuring and Testing the Impact of News on Volatility, Joumal of Finance, 48, 1749-1778.

EPPS, T.W. AND M. L. EPPS (1976), The Stochastic Dependence of Security Price Changes and Transaction Volumes: Implications for the Mixture of Distributions Hypothesis, Econometrica, 44, 305-321.

FAMA, E.F. (1965), The Behavior of Stock Market Prices, Joumal of Business, 38, 34-105.

FAMA, E.F. (1970), Efficient Capital Markets: A Review of Theory and Empirical Work, Journal of Finance, $25,383-417$.

FAMA, E.F. (1991), Efficient Capital Markets: II, Journal of Finance, 46, 1575-1617.

FOSTER, F.D. AND S. VISWANATHAN (1990), A Theory of Intraday Variations in Volume, Variance and Trading Costs in Securities Markets, Review of Financial Studies, 3, 593-624.

FOSTER, F.D. AND S. VISWANATHAN (1993A), Variations in Trading Volume, Returns, Volatility and Trading Costs: Evidence on Recent Price Formation Models, Joumal of Finance, $48,187-211$.

FOSTER, F.D. AND S. VISWANATHAN (1993B), The Effect of Public Information and Competition on Trading Volume and Price Vollatility, Review of Financial Studies, 6 , 23-56.

FOSTER, F.D. AND S. VISWANATHAN (1994), Strategic Trading with Asymmetrically Informed Traders and Long-Lived Information, Journal of Financial and Quantitative Analysis, 29, 499-518.

FOSTER, F.D. AND S. VISWANATHAN (1995), Can Speculative Trading Explain the Volume-Volatility Relation?, Joumal of Business and Economic Statistics, 13, 379-396. 
FRENCH, K.R. AND R. ROLL (1986), Stock Return Variances: The Arrival of Information and the Reaction of Traders, Journal of Financial Economics, 17, 5-26.

FRENCH, K.R., G.W. SCFWERT, AND R.F. STAMBAUGH (1987), Expected Stock Returns and Volatility, Journal of Financial Economics, 19, 3-29.

GALLANT, AR., D. HSIEH, AND G.E. TAUCHEN (1994), Estimation of Stochastic Volatility Models with Diagnostics, Unpublished Manuscript, Duke University.

GALLANT, A.R., P.E. ROSST, AND G.E. TAUCHEN (1992), Stock Prices and Volume, Review of Financial Studies, 5, 199-242.

GALLANT, A.R, P.E. ROSSI, AND G.E. TAUCHEN (1993), Nonlinear Dynamic Structures, Econometrica, 61, 871-907.

GALLANT, A.R. AND G.E. TAUCHEN (1996), Which Moments to Match?, Economic Theory, 12, 657-681.

GARMAN, M. (1976), Market Microstructure, Journal of Financial Economics, 3, 257-275.

GEWEKE, J. (1986), Modeling the Persistence of Conditional Variances: A Comment, Econometric Reviews, 5, 57-61.

GEWEKE, J. (1994), Monte Carlo Simulation and Numerical Integration, Working Paper 526, Federal Reserve Bank of Minneapolis.

GHYSELS, E. AND J. JASIAK (1994), Stochastic Volatility and Time Deformation: an Application of Trading Volume and Leverage Effects, Unpublished Manuscript, University of Montreal.

GLOSTEN, L.R., R. JAGANNATHAN, AND D.E. RUNKLE (1993), On the Relation between the Expected Value and the Volatility of the Nominal Excess Return on Stocks, Journal of Finance, 48, 1779-1801.

GLOSTEN, L.R., AND P.R. MiLgrom (1985), Bid, Ask, and Transaction Prices in a Specialist Market with Heterogeneously Informed Traders, Journal of Financial Economics, 14, 71-100.

GREGORY, A. (1989), A Non-Parametric Test for Autoregressive Conditional Heteroskedasticity: A Markov-Chain Approach, Journal of Business and Economic Statistics, 7, 307-317.

Hamilton, J.D. (1994), Time Series Analysis, Princeton University Press.

HANSEN, B.E. (1992), Autoregressive Conditional Density Estimation, Rochester Centre for Economic Research, Working Paper No. 322.

HARRIS, L.E. (1986), Cross-Security Tests of the Mixture of Distributions Hypothesis, Journal of Financial and Quantitatioe Analysis, 21, 39-46.

HARRIS, L.E. (1987), Transaction Data Tests of the Mixture of Distributions Hypothesis, Journal of Financial and Quantitative Analysis, 22, 127-141.

HARRIS, M. AND A. RAVIV (1993), Differences of Opinion Make a Horse Race, Review of Financial Studies, 6, 473-506.

HARVEY, A.C. (1989), Forecasting, Structural Time Series Models and the Kalman Filter Model, Cambridge University Press, Cambridge.

HARVEY, A.C., E. RUIZ, AND N. SHEPHARD (1994), Multivariate Stochastic Variance Models, Review of Economic Studies, 61, 247-264.

HARVEY, A.C. AND N. SHEPHARD (1994), Estimation of an Asymmetric Stochastic Volatility Model for Asset Returns, Unpublished Manuscript, Nuffield College, Oxford. 
HIEMSTRA, C. AND J.D. JONES (1994), Testing for Linear and Nonlinear Granger Causality in the Stock Price-Volume Relation, Journal of Finance, 49, 1639-1664.

HigGins, M.L... AND A.K. BerA (1992), A Class of Nonlinear ARCH Models, International Economic Review, 33, 137-158.

Holden, C.W. AND A. SubrahmanYAM (1992), Long-Lived Private Information and Imperfect Competition, Journal of Finance, 47, 247-270.

HOLTHAUSEN, R.W. AND R.E. VerReCCHIA (1990), The Effect of Informedness and Consensus on Price and Volume Behavior, Accounting Review, 65, 191-208.

HsIEH, D.A. (1989), Modeling Heteroskedasticity in Daily Foreign-Exchange Rates, Journal of Business and Economic Statistics, 7, 307-317.

HuFMAN, G. (1987), A Dynamic Equilibrium Model of Asset Prices and Transaction Volume, Journal of Political Economy, 95, 138-159.

HuFMAN, G. (1992), Information, Asset Prices and the Volume of Trade, Journal of Political Economy, 4, 1575-1590.

HuLL, J.C. AND A. WHITE (1987), The Pricing of Options on Assets with Stochastic Volatilities, Journal of Finance, 42, 281-300.

JACQuier, E., N.G. POLSON, AND P.E. Rossi (1994), Bayesian Analysis of Stochastic Volatility Models, Journal of Economics and Business Statistics, 12, 371-417, with discussion.

JAIN, P. AND G. JOH (1988), The Dependence between Hourly Prices and Trading Volume, Joumal of Financial and Quantitative Analysis, 23, 269-282.

JenNINGS, R., L. STARKS AND J. Fellingham (1981), An Equilibrium Model of Asset Trading with Sequential Information Arrival, Journal of Finance, 36, 143-161.

JONES, C., G. KAUL, AND M. LIPSON (1994), Transaction, Volume and Volatility, Review of Financial Studies, 4, 631-651.

JONG DE, P. AND N.SHEPHARD (1995), The Simulation Smoother for Time Series Models, Biometrika, 82, 339-350.

JORION, P. (1988), On Jump Processes in the Foreign Exchange and Stock Markets, Review of Financial Studies, 1, 427-445.

JUNG, $\mathbb{R}$. AND R. LIESENFELD (1996), Testing the Bivariate Mixture Hypothesis using German Stock Market Data, Unpublished Manuscript, University of Tübingen.

KARPOFP, J.M. (1986), A Theory of Trading Volume, Journal of Finance, 41, 1069-1087.

Karporf, J.M. (1987), The Relation between Price Changes and Trading Volume: A Survey, Joumal of Financial and Quantitative Analysis, 22, 109-126.

KIEFER, N. AND M. SALMON (1983), Testing Normality in Econometric Models, Economics Letters, 11, 123-127.

$\mathrm{KIM}_{*}$ S. AND N. SHEPHARD (1994), Stochastic Volatility: Optimall Likelihood Inference and Comparison with ARCH Models, Unpublished Manuscript, Nuffield College, Oxford.

KOEDIJK, K.G., F.G.J.A. NISSEN, P.C. SCHOTMAN AND C.C.P. WOLFF (1994), The Dynamics of Short-Term Interest Rate Volatility Reconsidered, LIFE Working Paper, University of Limburg, Maastricht.

KoOPMAN, S.J. (1993), Disturbance Smoother for State Space Models, Biometrika, 80, 117-126.

KYLE, A.S. (1985), Continuous Auctions and Insider Trading, Econometrica, 53, 1315 1335. 
LAMOUREXX, C.G. AND W. D. LASTRAPES (1990A), Heteroskedasticity in Stock Return Data: Volume versus GARCH Effects, Joumal of Finance, 45, 221-229.

LAMOUREUX, C.G. AND W.D. LASTRAPES (1990B), Persistence in Variance, Structural Change and the GARCH Model, Journal of Bustress and Ecomomic Statistics, 225-234.

LAMOUREUX, C.G. AND W. D. LASTRAPES (1994), Endogenous Trading Volume and Momentum in Stock-Return Volatility, Journal of Business and Economic Statistics, 12, 253-260.

LASTRA.PES, W.D. (1989), Exchange Rate Volatility and U.S. Monetary Policy: An ARCH Application, Journal of Money, Credit, and Banking, 21, 66-77.

LAUX, P. AND L. NG (1993), The Sources of GARCH: Empirical Evidence from an Intra Day Returns Model Incorporating Systematic and Unique Risks, Journal of International Money and Finance, 12,543-560.

LEBARON, B. (1993), The Joint Dynamics and Stability of Stock Prices and Volume, Unpublished Manuscript, University of Wisconsin.

LIESENFELD, $\mathbb{R}$ (1996), Dymamic Bivariate Mixture Models: Modelling the Behaviour of Prices and Trading Volume, Unpublished Manuscript, University of Tübingen.

LJUNG, G. AND G. Box (1978), On a Measure of Lack of Fit in Time Series Models, Biometrika, 65, 297-303.

LOCKE, P.R. AND C.L. SAYERS (1993), Intra-Day Futures Price Volatility, Joumal of Applied Econometrics, 8, 15-30.

LÜTKEPOHL, H. (1991), Introduction to Multiple Time Series Analysis Model, SpringerVerlag, Berlin.

MAHIEU, R. (1995), Financial Market Volatility: Statistical Models and Empirical Analysis, University Press, University of Limburg, Maastricht.

MAHIEU, R.J. AND P.C. SCHOTMAN (1996), An Empirical Application of Stochastic Volatility Models, Unpublished Manuscript, University of Limburg, Maastricht.

MANDELBROT, B. (1963), The Variation of Certain Speculative Prices, Journal of Business, 36, 394-419.

MANDELBROT, B. AND H.M. TAYLOR (1967), On the Distribution of Stock Price Differences, Operations Research, 15, 1057-1062.

MCGulrk, A. J. RObERTSON, AND A. SPANOS (1993), Modeling Exchange Rate Dynamics: Nonlinear Dependence and Thick Tails, Econometric Review, 12, 33-63.

MEESE, R.A. AND K. ROGOFF (1983), Empirical Exchange Rate Models of the Seventies: Do They Fit out of Sample?, Jourmal of International Economics, 14, 3-24.

MElino,A. AND S.M. TURNBull (1990), Pricing Foreign Currency Options with Stochastic Volatility, Jowrnal of Econometrics, 45, 239-265.

Merton, R.C. (1990), Continuous-Time Finance, Blackwell, Cambridge, Massachusetts.

Milgrom, P. AND N. STOKEX (1982), Information, Trade, and Common Knowledge, Jonmal of Economic Theory, 26, 17-27.

MORSE, D. (1980), Asymmetrical Information in Securities Markets and Trading Volume, Journal of Financial and Quantitative Analysis, 15, 1129-1148.

Nalson, D.B. (1989), Madeling Stock Market Volatility Changes, Proceedings from the American Statistical Association.

NELSON, D.B. (1990), ARCH Models as Diffusion Approximations, Joumal of Econometrics, 45, 7-38. 
NeLSON, D.B. (1991), Conditional Heteroskedasticity in Asset Pricing: A New Approach, Econometrica, 59, 347-370.

NEL SON, D.B. (1992), Filtering and Forecasting with Misspecified ARCH Models I: Getting the Right Variance with the Wrong Model, Journal of Ecomometrics, 52, 6190.

NiEuwland, F. (1993), Speculative Market Dynamics: An Econometric Analysis of Stock Market and Foreign Exchange Market Dymamics, University Press, University of Limburg, Maastricht.

O'HARA, M. (1995), Market Microstructure Theory, Blackwell Business.

OSBORNE, M.F.M. (1959), Brownian Motion in the Stock Market ${ }_{*}$ Operation Research, 7 , 145-173.

PAGAN, A.R. (1993), The Econometrics of Financial Markets, Unpublished Manuscript, Australian National University.

PAGAN, A.R. AND A.D. HAll (1983), Diagnostic Tests as Residual Analysis, Australian National University.

PAGAN, A.R. AND A. UlLAH (1988), The Econometric Analysis of Models with $\mathbb{R}$ isk Terms, Journal of Applied Econometrics, 3, 87-105.

PfleIDERER, P. (1984), The Volume of Trade and the Variability of Prices: A Framework for Analysis in Noisy Rational Expectations Equilibria, Working Paper, Stanford University.

PITT, M. AND N.SHEPHARD (1996), Analytic Convergence Rates and Parametrization Issues for the Gibbs Sampler Applied to State Space Models, Working Paper, Nuffield College, Oxford.

RICHARDSON, G., S. SEFCIK AND R. THOMPSON (1986), A Test of Dividend Irrelevance Using Volume Reactions to a Change in Dividend Volume, Journal of Financial Economics, 17, 313-333.

RICHARDSON, M. AND T. SMITH (1994), A Direct Test of the Mixture of Distributions Hypothesis: Measuring the Daily Flow of Information, Journal of Financial and Quantitative Analysis, 29, 101-116.

RuIz, E. (1994), Quasi-Maximum Likelihood Estimation of Stochastic Volatility Models, Journal of Econometrics, 63, 289-306.

SHEPARD, N. (1994), Partial Non-Gaussian State Space, Biometrika, 81, 115-131.

SHEPARD, N. AND M.K. PITT (1995), Likelihood Analysis of Non-Gaussian Parameter

Driven Models, Unpublished Manuscript, Oxford University.

SIMS, C.A. (1980), Macroeconomics and Reality, Econometrica, 48, 1-48.

SMIRLOCK, M. AND L. STARKS (1988), An Empirical Analysis of the Stock Price-Volume Relationship, Journal of Banking and Finance, 12, 31-41.

SPANOS, A. (1991), A Parametric Approach to Heteroskedasticity: The Student's $t$ and Elliptic Linear Regression Models, Unpublished Manuscript, Virginia Tech.

SPANOS, A. (1992), The Student's $t$ Autoregressive Model with Dynamic Heteroskedasticity, Unpublished Manuscript, Virginia Tech.

STOLL, H. (1978), The Supply of Dealer Services in Securities Markets, fournal of Finance, 33, 1131-1151.

StOLL, H., AND R. WHALEX, (1990), Stock Market Structure and Volatility, Review of Financial Studies, 3, 37-71. 
TAuChEN, G.E. AND M. PTtTS (1983), The Price Variability-Volume Relationship on Speculative Markets, Econometrica, 2, 485-505.

TAUCHEN, G.E., H. ZHANG, AND M. LHU (1993), Volume, Volatility and Leverage: A Dynamic Analysils, Unpublished Manuscript, Duke University.

TAYLOR, S.J. (1986), Modelling Financial Time Series, Wiley, New York.

TAYLOR, S.J. (1994), Modeling Stochastic Volatility: a Review and Comparative Study, Mathematical Finance, 4, 183-204.

TreRNEY, L. (1994), Markov Chains for Exploring Posterior Distributions, Anrals of Statistics, 21, 1701-1762.

WANG, J. (1994), A Model of Competitive Stock Trading Volume, Journal of Political Economy, 102, 127-168.

WEIss, A.A. (1986), Asymptotic Theory for ARCH Models" Estimation and Testing, Econometric Theory, 2, 107-131.

Westerfelel, R. (1977), The Distribution of Common Stock Price Changes: An Application of Transaction Time and Subordinated Stochastic Models, Journal of Financial and Quantitative Analysis, 12, 743-765.

WolF, C.C.P. (1987), Forward Foreign Exchange Rates, Expected Spot Rates and Premia: A Signal - Extraction Approach, Journal of Finance, 42, 395-406.

WOOD, R., T. MCINISH AND J. ORD (1985), An Investigation of Transaction Data for NYSE Stocks, Journal of Finance, 40, 723-741.

YING, C.C (1966), Stock Market Prices and Volume of Sales, Econometrica, 34, 676-685. 


\section{Nederlandse Samenvatting}

In de beleggingswereld wordt algemeen aangenomen dat een beter inzicht in de variabiliteit of volatiliteit van financiële markten leidt tot professionelere beleggingsbeslissingen. Financiële instellingen als bijvoorbeeld pensioenfondsen en verzekeringsmaatschappijen streven naar een efficiënte allocatie van middelen over de aanwezige beleggingscategorieën. Met name in continentaal Europa wordt derhalve steeds meer in risicodragend kapitaal geïnvesteerd. Van oudsher grote, inefficiënte posities in vastrentende beleggingen worden massaal in aandelen herbelegd. In dit dynamisch allocatieproces is het van eminent belang dat we de kenmerken van de verschillende beleggingscategorieën nader bestuderen. Volatiliteit, als maatstaf voor risico, is één van de belangrijkste parameters voor een groot aantal beslissingen op dit gebied. De grootte van een geschikte bandbreedte rond een strategische benchmark voor asset allocatie is een functie van de volatiliteit van de betreffende beleggingscategorie. Ook de in opkomst zijnde risicobeheersingsmethodieken, zoals bijvoorbeeld Value at Risk (VAR), zijn gebaseerd op veronderstellingen omtrent het volatiliteitsproces van de betreffende beleggingen. Tijdsvariërende risicopremies kunnen immers van grote invloed zijn op de uitkomsten en consequenties van deze studies. Een laatste voorbeeld waar volatiliteit een belangrijke rol speelt is de optie-theorie: de volatiliteit van de onderliggende waarde is een belangrijke factor bij de waardering van derivaten. In deze dissertatie bestuderen wij uitsluitend de volatiliteit van aandelenmarkten.

Het empirisch onderzoek naar de volatiliteit van aandelenmarkten beperkte zich tot voor kort tot het schatten van univariate tijdreeksmodellen. De heteroskedastische ARCH modellen van Engle (1982) en alle aanverwante specificaties waren hierin zeer succesvol. Deze dissertatie verbreedt de discussie over het onderliggend proces dat aandelenrendementen genereert. In de eerste twee hoofdstukken introduceerden wij de onderzoeksopzet en gaven wij een overzicht van de relevante literatuur. In de daaropvolgende hoofdstukken hebben wij voor enkele grote aandelenmarkten met behulp van twee klassen van modellen, (G)ARCH en Stochastic Volatility (SV), persistentie in de volatiliteit van aandelenrendementen aangetoond. Bovendien introduceerden wij een in onze ogen zeer belangrijke variabele: handelsvolume of het aantal verhandelde aandelen. Het gebruik van handelsvolume in de univariate modellen was redelijk succesvol, maar de univariate modelstructuur was niet 
geheel consistent met de in Hoofdstuk 2 geïntroduceerde Mixture of Distributions Hypothesis (MDH). Derhalve concentreerden wij ons in het laatste deel van deze dissertatie voornamelijk op een bivariate modelstructuur. Naast de discussie over de relatie tussen de volatiliteit van aandelenrendementen en handelsvolume was de te hanteren schattingsprocedure indirect een belangrijk aandachtspunt. In deze samenvatting laten wij de belangrijkste empirische bevindingen de revue passeren.

In Hoofdstuk 3 introduceerden we de standaard GARCH methodologie en een aantall verwante univariate volatiliteitsmodellen. Deze populaire klasse van modellen veronderstelt een autoregressieve structuur van de conditionele variantie, hetgeen impliceert dat een schok in de volatiliteit langere tijd persisteert. In de empirische literatuur werden verschillende conditionele verdelingen voor de storingsterm in een GARCH-model verondersteld. Wij schatten voor de Frankfurt Stock Exchange enkele GARCH tijdreeksmodellen met twee mogelijke conditionele verdelingen. De empirische resultaten voor wekelijkse en maandelijkse index-rendementen tonen aan dat de student $t$ verdeling doorgaans een betere keuze is dan de normale verdeling. De geschatte GARCH $t$-modellen zijn vooral succesvol in het verwijderen van scheefheid, dikstaartigheid en heteroskedasticiteit in de onderliggende aandelenrendementen. Deze resultaten bevestigen de bevindingen van eerdere onderzoeken voor de Amerikaanse aandelenmarkt, zie o.a. Bollerslev e.a. (1992).

De volgende stap in ons onderzoek was gebaseerd op de inzichten van de MDH van Clark (1973). Deze hypothese veronderstelt een gezamenlijke afhankelijkheid van rendementen en handelsactiviteit, i.e. volume en dus transacties, op aandelenmarkten. In Hoofdstuk 4 veronderstellen wer geinspireerd door eerder onderzoek in de Verenigde Staten van Lamoureux en Lastrapes (1990a), dat de mate van handelsactiviteit in de aandelenmarkt een goede benadering is voor het latent aanwezige en niet waarneembare informatie-proces dat rendementen genereert. Het additief toevoegen van dagelijks handelsvolume of het dagelijks aantal transacties in de conditionele variantie-vergelijking van een GARCH-model resulteerde in een dalende persistentie van de volatiliteit, hetgeen in overeenstemming is met de resultaten van Lamoureux en Lastrapes (1990a). De parameters voor handelsactiviteit waren bovendien statistisch significant. Wij dragen bij aan het werk van Lamoureux en Lastrapes (1990a) door het veronderstellen van een $t$-verdeling voor de return-schokken en door gebruik te maken van een Duitse (DAX) data set van rendementen, volumes en aantal transacties. Vervolgens hebben we aangetoond dat deze relatief eenvoudige, univariate modelstructuur enkele theoretische en empirische tekortkomingen heeft. Wij introduceerden derhalve een alternatief, multiplicatief GARCH-model, waarin we dagelijks handelsvolume interpreteerden als een schaalfactor voor de volatiliteit. In de 
output van de multiplicatieve modellen vinden we in tegenstelling tot de additieve modellen geen daling in de persistentie na het meeschatten van volume.

Hoofdstuk 5 is voornamelijk gewijd aan stochastische volatiliteitsmodellen. We hebben aangetoond dat een SV-framework voor rendementen consistent is met de $\mathrm{MDH}$. In deze modellen interpreteren wij dagelijks handelsvolume evenals in Hoofdstuk 4 als schaalfactor voor volatiliteit. De empirische resultaten voor een steekproef van Amerikaanse aandelen, eveneens gebruikt in een onderzoek van Lamoureux en Lastrapes (1994), tonen aan dat volume een zeer belangrijke variabele is bij de verklaring van het return-proces. In vrijwel elke schatting leidde het gebruik van volume als schaalfactor tot betere schattingsresultaten, terwijl de persistentie in de volatiliteit niet daalde. Granger causality tests en vector-autoregressieve schattingen in dit hoofdstuk bevestigen eveneens de sterke onderlinge afhankelijkheid van rendementen, volatiliteit en activiteit op de beursvloer. De conclusie van dit hoofdstuk is dat de MDH in principe wijst op een bivariaat model: zowel aandelenrendementen als handelsvolume worden simultaan beïvloed door hetzelfde latent aanwezige informatie-proces. Derhalve richtten we onze aandacht in het laatste deel van deze dissertatie met name op bivariate modellen met een sterke link met de $\mathrm{MDH}$. De resultaten voor het hefboomeffect, besproken in de inleidende hoofdstukken, waren minder eenduidig.

Hoofdstuk 6 voegt op meerdere vlakken nieuwe dimensies toe aan deze dissertatie, o.a. door de introductie van enkele bivariate specificaties en het gebruik van intra-daily data voor de Amsterdamse aandelenmarkt. Daarnaast gebruikten we een relatief nieuwe schattingsmethode, te weten Simulated Maximum Likelihood Estimation. De patronen in intra-daily volatiliteit en handelsvolume kwamen overeen met de resultaten van studies in andere landen. De bivariate specificatie voor rendementen en handelsvolume in dit hoofdstuk werd oorspronkelijk voorgesteld door Tauchen en Pitts (1983). In deze studie namen ze aan dat het niet waarneembare, latente informatie-proces serieel onafhankelijk was. We schatten hun modelspecificatie zowel onder de aanname van een onafhankelijk (statisch) als van een afhankelijk (dynamisch) proces. De empirische resultaten in dit hoofdstuk geven duidelijk aan dat de dynamische specificaties een betere optie zijn. Het is echter van belang dat we zeer voorzichtig zijn met het hanteren en interpreteren van intra-daily data vanwege markt-microstructurele en institutionele factoren. Het modelleren van deze hoog-frequente data staat nog relatief in zijn kinderschoenen en is derhalve een interessant gebied voor toekomstig onderzoek.

In Hoofdstuk 7 introduceerden we een bivariate specificatie voor dagelijkse rendementen en handelsvolumes gebaseerd op een markt-microstructuur 
model van Glosten en Milgrom (1985). Dit model wordt voornamelijk gekenmerkt door de aanwezigheid van asymmetrische informatie en zogenaamde liquidity traders. Deze gemodificeerde versie van de MDH werd door Andersen (1996) gelanceerd. In dit model is een deel van het dagelijks handelsvolume onafhankelijk van het latente informatie-proces (liquidity trading), terwijl het andere deel afhankelijk is van het latente proces (information trading). Wij dragen op verschillende fronten bij aan het werk van Andersen (1996). We hanteerden een andere schattingsmethode, te weten een Markov Chain Monte Carlo (MCMC) methode gebaseerd op Bayesiaanse analyse, terwiil Andersen (1996) een GMM-benadering koos. MCMC heeft het voordeel dat het een schatting van het latente proces oplevert. Deze schatting kan worden ingezet in verschillende gebieden van de financiële economie, bijvoorbeeld dynamische allocatie en optie-waardering. Het belangrijkste schattingsresultaat van dit hoofdstuk is dat we, in tegenstelling tot eerder onderzoek van Andersen (1996) en Liesenfeld (1996) en onze resultaten in Hoofdstuk 6, geen daling van de persistentie in het bivariate model vinden. Dit is volledig in overeenstemming met de $\mathrm{MDH}$. We vermoeden dat de keuze van de schattingsmethode in dit kader van invloed is. De Bayesiaanse schattingsmethodieken dienen naar onze mening in de nabije toekomst nader bestudeerd te worden.

Deze dissertatie heeft op verschillende wijzen bijgedragen aan de bestaande literatuur op het gebied van de relatie tussen aandelenrendementen, volatiliteit en handelsvolume. Met name het bivariate model in het vorige hoofdstuk lijkt een zeer interessant startpunt voor nadere onderzoeksprojecten. De empirische resultaten in deze dissertatie geven duidelijk aan dat handelsvolume een belangrijke variabele is bij het bestuderen van het niet-waarneembare proces dat aandelenrendementen genereert, zij het in een univariaat of in een bivariaat framework. Een bivariate modelstructuur voor aandelenrendementen en handelsvolume is theoretisch meer in overeenstemming met de intuitief aantrekkelijke MDH. Een ander interessant gebied voor toekomstig onderzoek is het onderscheiden van verschillende typen informatie in financiële markten. Een laatste mogelijk aandachtspunt is het verder ontwikkelen van modellen voor het verklaren van intra-daily rendementen en volumes. Een beter zicht op hoog-frequente prijs en volume data leidt ons inziens tot een beter begrip van de werking van aandelenmarkten in het algemeen. 


\section{Curriculum Vitae}

Rob Bauer, geboren 19 augustus 1967 te Heerlen, slaagde in 1985 voor het eindexamen Gymnasium-beta aan het Bernardinuscollege te Heerlen. Vervolgens studeerde hij Economie aan de Universiteit van Maastricht. In 1990 behaalde hij zijn doctoraaldiploma. Van september 1990 tot en met augustus 1992 was hij toegevoegd docent bij de sectie Financiering van de Universiteit Maastricht. Van september 1991 tot en met juli 1995 was hij wetenschappelijk medewerker bij de sectie Financiering van de Rheinisch-Westfälisch Technische Hochschule in Aken. Hier werkte hij aan verschillende onderzoeksprojecten, o.a. voor Inquire Europe en de Deutsche Forschungsgemeinschaft (DFG), waarvan dit proefschrift het resultaat is. In december 1992 behaalde hij een Master of Financial Economics aan het Tilburgs Instituut voor Academische Studies (TIAS). Vanaf augustus 1995 is hij werkzaam als portefeuille manager Aandelen bij de Stichting Pensioenfonds ABP in Heerlen.

Rob Bauer, born August 19th 1967 in Heerlen, studied Economics at the University of Maastricht from 1985 until 1990. He received his Master's degree in Economics and Business Administration in April 1990. He was assistant professor at the Finance department of the University of Maastricht from September 1990 to August 1992. From September 1991 to Jully 1995 he worked for the Finance Department of the Rheinisch-Westfälisch Technische Hochschule in Aachen, Germany. There he started working on his thesis and several other research projects. In December 1992 he received a Master's degree in Financiall Economics at the Tilburg Institute for Academic Studies (TIAS). Starting August 1995, he is working as a portfolio manager at the Equity Investments Department of the Stichting Pensioenfonds ABP in Heerlen. 